\title{
Chip Based Common-path Swept-source Optical Coherence Tomography Device
}

\section{Lantian Chang}




\section{Chip Based Common-path Swept-source Optical Coherence Tomography Device}

Lantian Chang

2016 


\section{Members of the thesis committee:}

\begin{tabular}{|c|c|c|}
\hline Prof. dr. ir. & J.W.M. Hilgenkamp & University of Twente (chairman and secretary) \\
\hline Prof. dr. & V. Subramaniam & University of Twente (promotor) \\
\hline Dr. ir. & J.S. Kanger & University of Twente (co-promotor) \\
\hline Prof. dr. & A.M. Versluis & University of Twente \\
\hline Prof. dr. & W. Steenbergen & University of Twente \\
\hline Prof. dr. & A.G.J.M. van Leeuwen & $\begin{array}{l}\text { Academic Medical Center, } \\
\text { University of Amsterdam }\end{array}$ \\
\hline Prof. dr. & P. Bienstman & Ghent University \\
\hline Prof. dr. & A.P. Mosk & Utrecht University \\
\hline
\end{tabular}

The research described in this thesis was carried out at the Integrated Optical MicroSystems (IOMS) Group, Faculty of Electrical Engineering, Mathematics and Computer Science, and Nanobiophysics (NBP) Group, Faculty of Science and Technology, MESA+ Institute for Nanotechnology, University of Twente, P.O. Box 217, 7500 AE Enschede, The Netherlands.

The research was financially supported by the IOP Photonic Devices program (Low-cost handheld OCT device, IOP PD100019), managed by the Technology Foundation RVO.

\section{Cover: Power of light}

Light is a powerful tool to uncover secrets of the Universe. The front cover is a schematic of a parallel optical coherence tomography chip, which represents my research during the day in a microscopic world. The back cover is an image of the Orion nebula, which represents my hobby, astrophotography, during the evening in a cosmic world.

(The image of the Orion nebula was taken with a Meade Lx90 8' telescope, city light filter, Nikon D750 camera, ISO 100, Exp. 10 min.)

Cover designed by Ying Du and Lantian Chang

\section{Copyright $\odot 2016$ by Lantian Chang, Enschede, The Netherlands}

All rights reserved. No part of this book may be reproduced by any means without the prior written permission of the author.

ISBN: 978-90-365-4099-5

DOI: $10.3990 / 1.9789036540995$

URL: http://dx.doi.org/10.3990/1.9789036540995 


\title{
CHIP BASED COMMON-PATH SWEPT-SOURCE OPTICAL \\ COHERENCE TOMOGRAPHY DEVICE
}

\section{DISSERTATION}

\author{
to obtain \\ the degree of doctor at the University of Twente, \\ on the authority of the rector magnificus, \\ prof. dr. H. Brinksma \\ on account of the decision of the graduation committee, \\ to be publicly defended \\ on Wednesday $15^{\text {th }}$ of June 2016 at $14: 45 \mathrm{~h}$
}

by

\section{Lantian Chang}

Born on $14^{\text {th }}$ of April 1986

in Shandong, China 
This dissertation is approved by:

Prof. dr. V. Subramaniam

Dr. ir. J.S. Kanger
University of Twente (promotor)

University of Twente (co-promotor) 


\section{Table of contents}

\section{Introduction}

1.1 The goal of the thesis

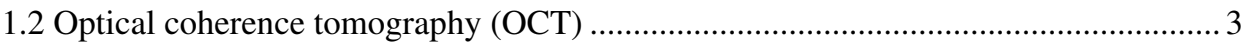

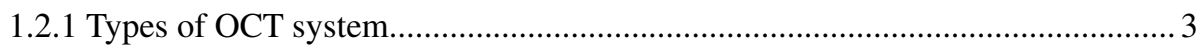

1.2.2 Important Fourier-domain OCT (FD-OCT) parameters ................................... 4

1.3 Literature survey of existing chip-based FD-OCT systems ................................... 7

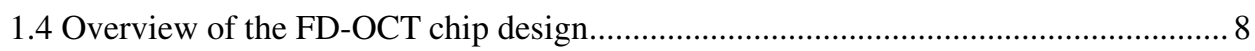

1.4.1 Components overview for on-chip functionality ......................................... 8

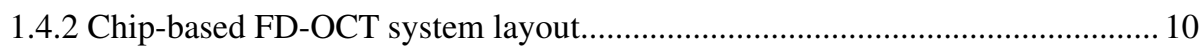

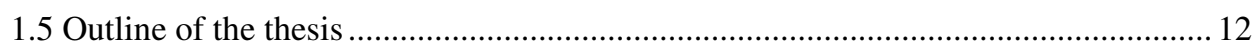

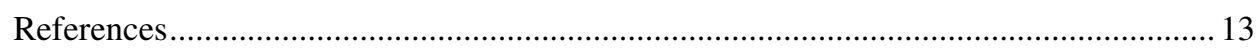

2 Mathematical model of a FD-OCT.......................................................... 17

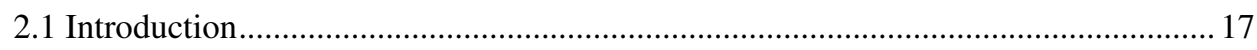

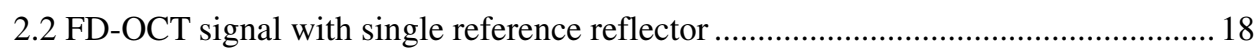

2.3 FD-OCT signal with multiple reference reflectors ................................................ 22

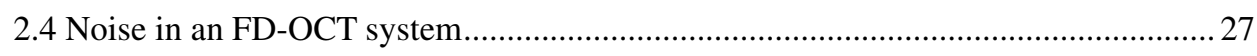

2.5 Signal-to-noise ratio (SNR) in an FD-OCT system.............................................. 29

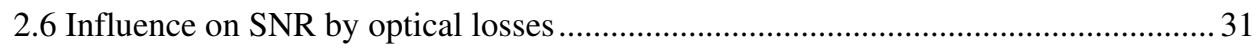

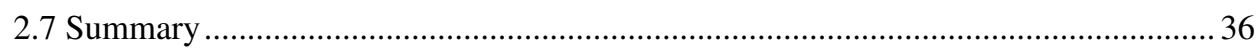

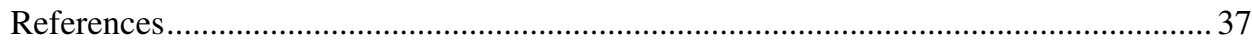

3 Design, fabrication and characterization of waveguide structures .... 39

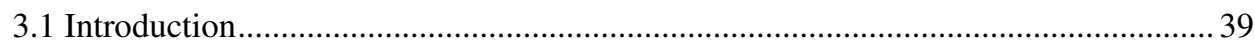

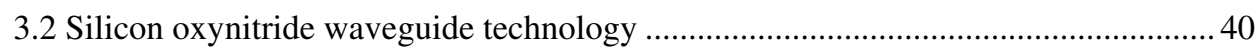

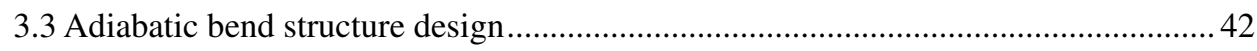

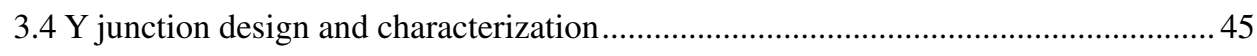

3.4.1 Modified blunt Y junction design ............................................................. 47

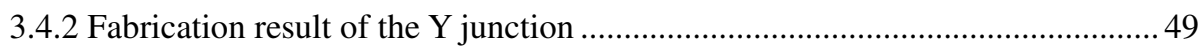

3.4.3 Y junction splitting ratio measurements ....................................................... 50

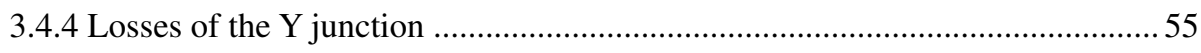

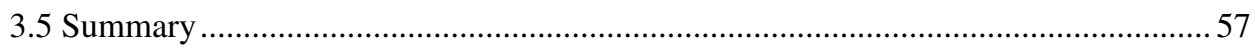

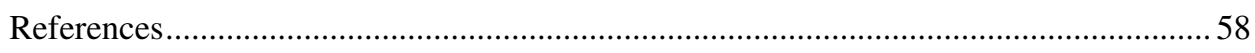




\section{Integrated micro-ball lens technology ................................................61}

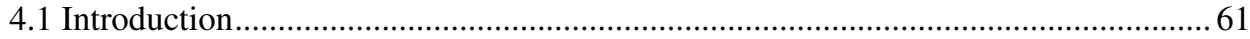

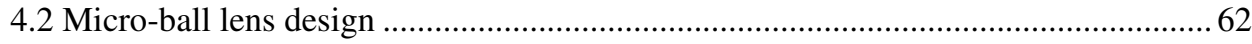

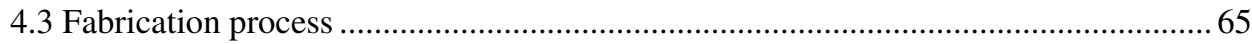

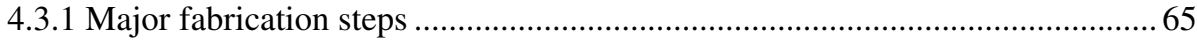

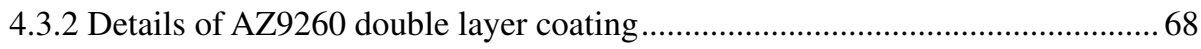

4.3.3 Details related to AZ9260 rehydration .............................................................. 72

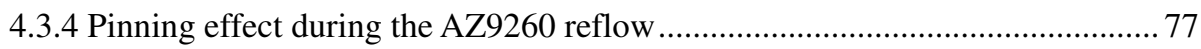

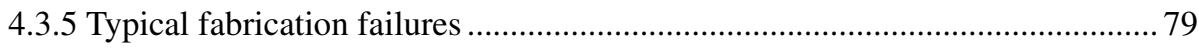

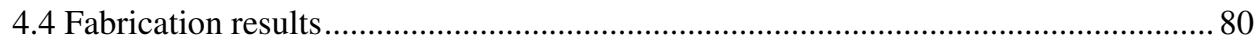

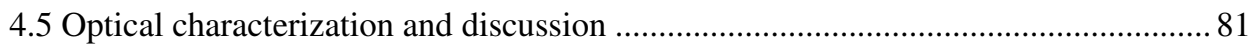

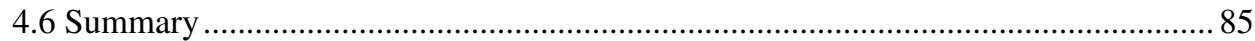

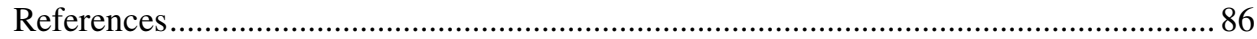

5 Single channel OCT measurements.......................................................... 87

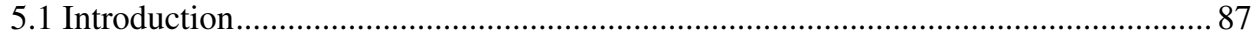

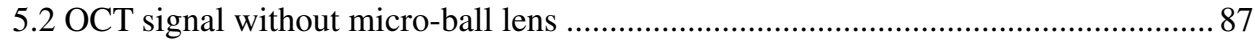

5.3 OCT signal enhancement with an on-chip micro-ball lens ................................... 92

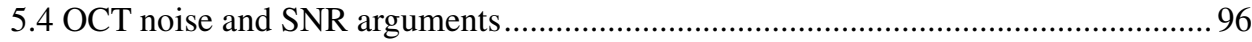

5.4.1 Suppression of the Fourier transform induced side-lobes with local mean

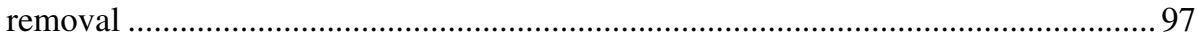

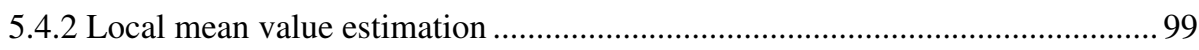

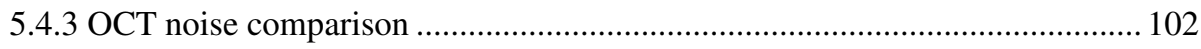

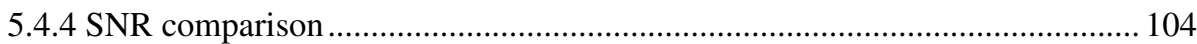

5.5 Performance comparison between a chip system with a micro-ball lens and a fiber

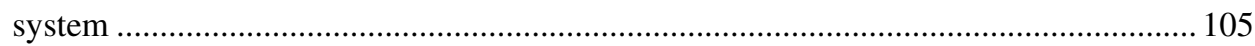

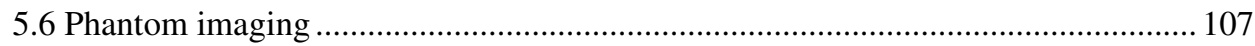

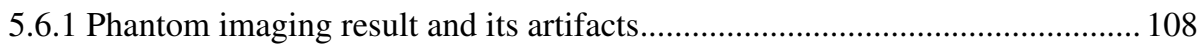

5.6.2 Multiple reference planes induced ghost images suppression.......................... 110

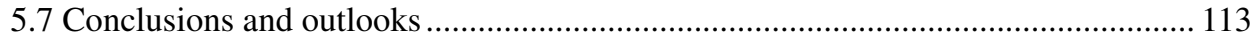

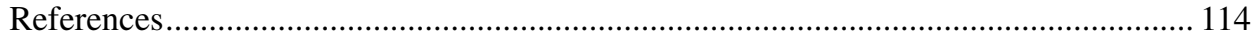

6 Parallel OCT on Chip ............................................................................... 115

6.1 Introduction of full-field OCT (FF-OCT) and parallel OCT (P-OCT) ................... 115

6.2 Chip-based parallel swept-source OCT (PSS-OCT) designs ................................ 117

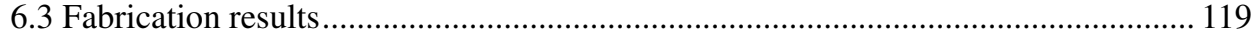

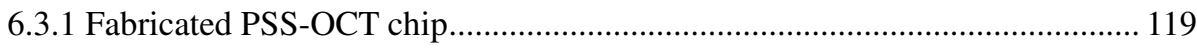


6.3.2 Possible PSS-OCT characterization in the future ......................................... 121

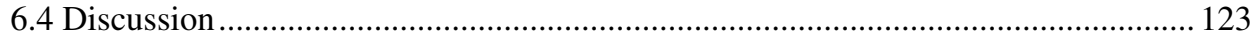

6.4.1 Possible layout to reduce channel spacing …................................................. 123

6.4.2 Possible solutions to avoid waveguide crossing in P-OCT ............................ 124

6.4.3 Suggestions to reduce motion blur with P-OCT.......................................... 127

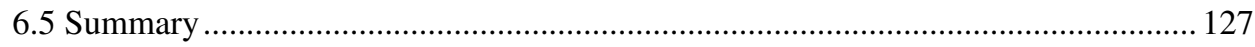

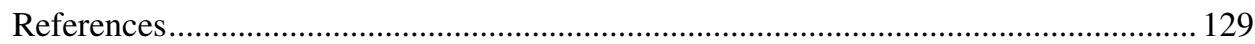

7 Conclusions and outlook................................................................... 131

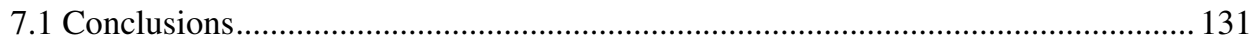

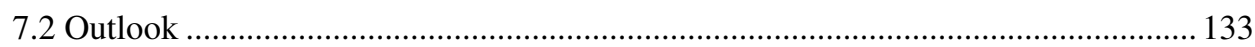

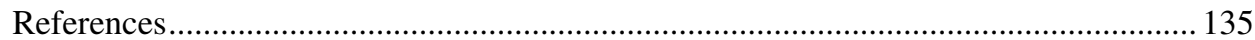

Appendices: Fabrication processes............................................................. 137

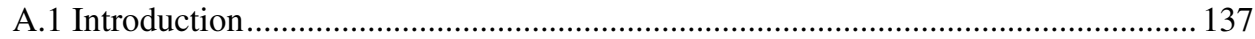

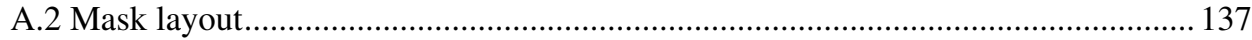

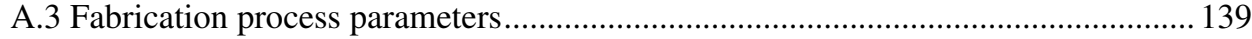

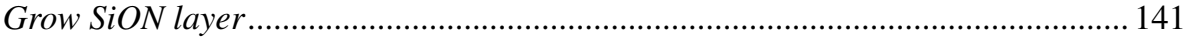

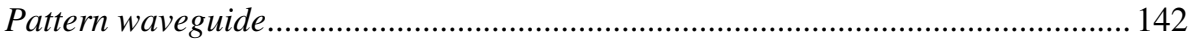

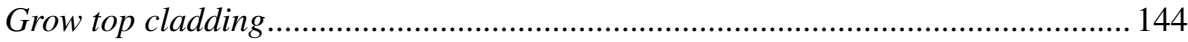

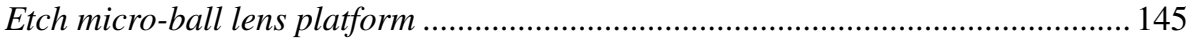

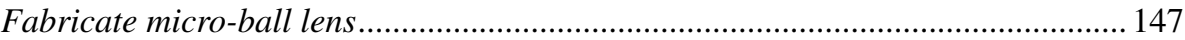

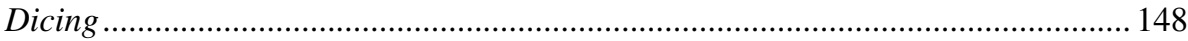

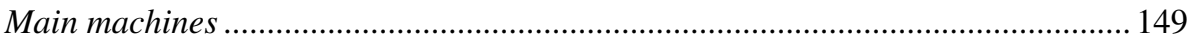

List of Abbreviations....................................................................... 151

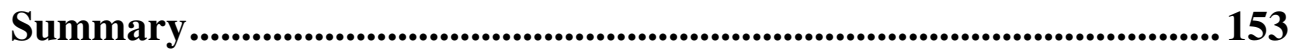

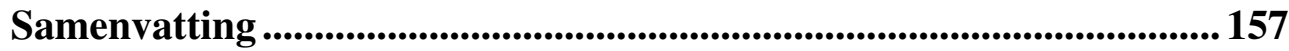

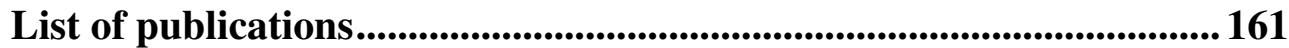

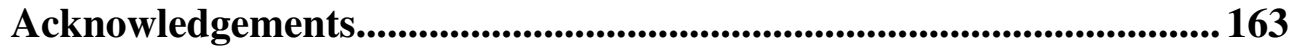




\section{Chapter 1}

\section{Introduction}

\subsection{The goal of the thesis}

In this thesis, we develop a chip-based optical coherence tomography (OCT) system. OCT is an optical imaging technique which provides three-dimensional images with micrometerresolution [1]. OCT has been extensively used as a medical imaging technology for disease diagnostics, treatment planning, and surgical guidance [2]. A comparison of penetration depth and resolution [3] between several commonly used medical imaging technologies is summarized in Fig. 1.1.1. OCT fills the gap between ultrasound imaging and confocal microscopy in terms of both penetration depth $(\sim 2-3 \mathrm{~mm})$ and resolution $(\sim 1-10 \mu \mathrm{m})$ [2]. These unique features of OCT make it a powerful imaging tool.

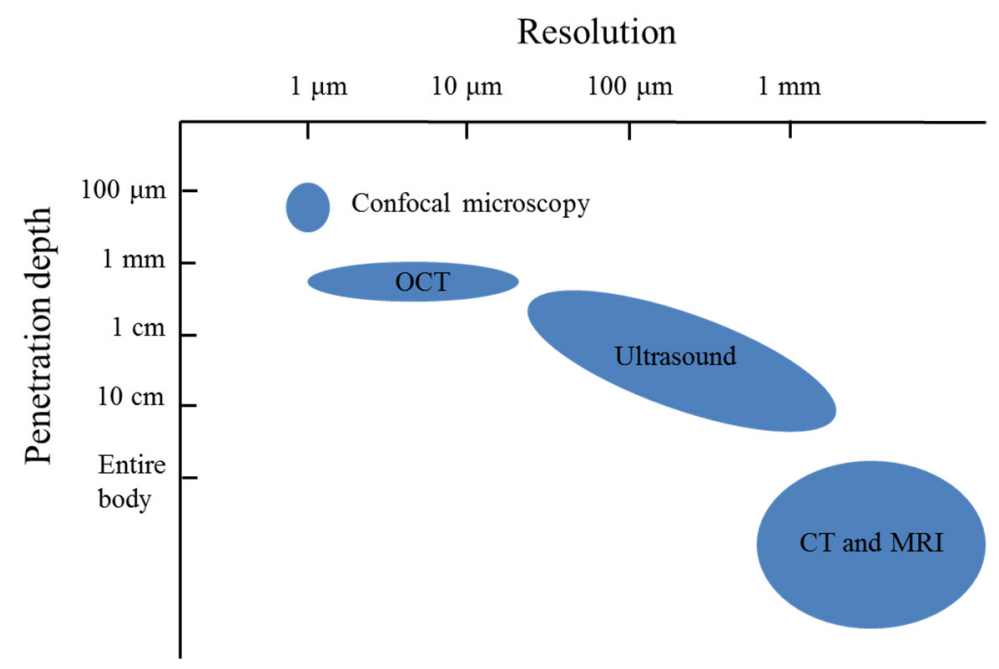

Fig. 1.1.1. Comparison of the penetration depth and resolution between several commonly used medical imaging techniques. CT: computed tomography; MRI: magnetic resonance imaging. 
After more than two decades of development since the first OCT system [1], this imaging technique has been widely used in clinical applications to measure different kinds of biological tissue such as skin [4] and teeth [5]. To date, OCT has its most successful contribution in the field of ophthalmology, where it is an important diagnostic technology in the areas of retinal diseases and glaucoma [6-9]. However, applications of OCT have expanded into many other medical fields during its development, such as cardiology [10], gastroenterology [11], gynecology [12], oncology [13], pulmonology [14] and urology [15]. In addition to clinical applications, OCT has also been increasingly used in industrial applications [16-20].

Currently, most of the OCT systems are based on discrete free-space optical components and optical fibers. These discrete components keep these instruments costly and bulky. The development in integrated optical circuit technology provides the opportunity to develop miniaturized, stable and maintenance-free OCT systems. In case of mass production, chipbased systems have the potential for considerable size- and cost- reduction, which may lead to wider applications.

The goal of this thesis is developing an OCT chip with external light source and detector. The developed chip is intended to be used in a hand-held OCT probe for skin applications. The light source and detector can be outside the probe and coupled to the chip through fibers.

In the rest of this chapter we give an overview of different types of OCT systems followed by a discussion on the key parameters that describe the performance of the OCT systems. Next, based on these key parameters, we provide a short discussion about the general criteria for the chip design. After that, we provide a literature survey on chip-based OCT systems to discuss their achievements and problems. In Section 1.4, we first provide a discussion of the functions needed on an OCT chip and an overview of the possible components to realize these functions. We then present the concept of our OCT chip design, which solves three common problems in previous chip-based OCT systems. The advantages and disadvantages of this design concept are discussed. Finally, the layout of this thesis is introduced. 


\subsection{Optical coherence tomography (OCT)}

\subsubsection{Types of OCT system}

OCT imaging is performed by measuring the interference between light scattered (or reflected) from a sample and light reflected from a reference mirror. There are two main types of OCT systems: time-domain OCT (TD-OCT) and Fourier-domain OCT (FD-OCT) as shown in Fig. 1.2.1. The working principle of both systems is introduced below.

(a): TD-OCT

\section{Reference Reflector}

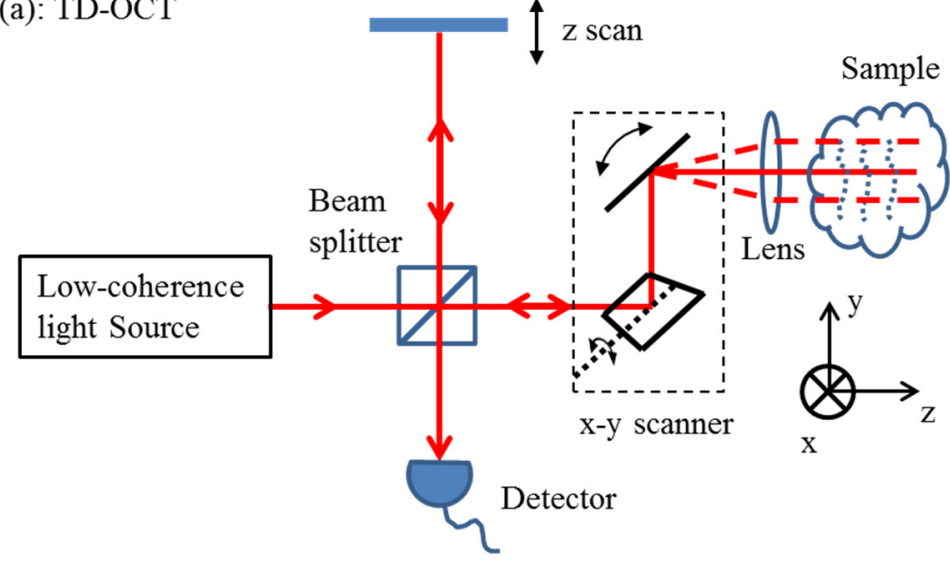

Reference Reflector (fixed)

(b): FD-OCT

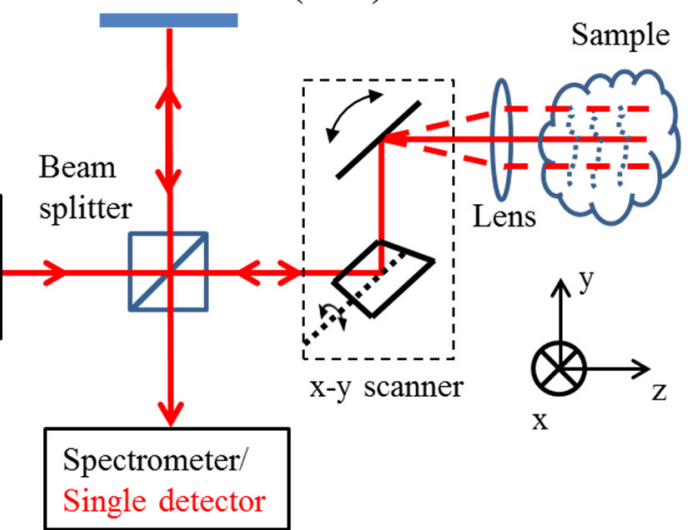

Fig. 1.2.1. Schematic of (a) TD-OCT and (b) FD-OCT. The $x$-y scanner is used to perform the B-scans and $\mathrm{C}$-scans. The combination of a low-coherence (broadband) light source and a spectrometer is called SD-OCT. The combination of a swept source and a single detector is called SS-OCT. 
TD-OCT uses a low-coherence light source. Interference at the detector is measured as a function of the reference reflector position. Interference is only apparent if the optical path length difference between the reference mirror and sample reflections (or scatters) is less than the coherence length of the light source (typical 1-10 $\mu \mathrm{m}$ ). A one-dimensional scattering or reflection profile of the sample is obtained by translating the reference reflector along the optical axis (known as axial or $\mathrm{z}$ direction) as a function of time, which is known as A-scan. A two- or three-dimensional image could be obtained by using an x-y scanner (such as a galvanometer scanner) to measure A-scans at multiple lateral locations on the sample which are known as B-scans and C-scans, respectively.

FD-OCT measures the interference as a function of light frequency with a fixed reference reflector. The A-scan is constructed by computing the Fourier transform of the measured interference spectrum (intensity at the detector as function of the frequency of the light). A detailed model and mathematical description of FD-OCT are presented in Section 2.2. FD-OCT is performed as either a spectral-domain OCT (SD-OCT) which employs a broad-band light source and a spectrometer or as a swept-source OCT (SS-OCT) which uses a narrow-bandwidth frequency-swept light source and a single detector [21]. Except for the light source and detector, the optical paths are the same for both SD-OCT and SS-OCT. The B-scans and C-scans are obtained in the same way as for TD-OCT.

The state-of-the-art OCT systems are based on FD-OCT [22], which provides a typical sensitivity advantage of 20-30 dB over TD-OCT [23]. Therefore, in this thesis, we have based our design on an FD-OCT system. The overall performance (e.g. imaging depth and resolution) of an FD-OCT system depends on many parameters that should be well chosen for a given application. In the next section we will discuss the key-parameters of the FDOCT system.

\subsubsection{Important Fourier-domain OCT (FD-OCT) parameters}

Several important parameters are commonly referred to in FD-OCT systems to quantify their imaging quality. These parameters are (i) the center wavelength; (ii) axial resolution; (iii) maximum imaging depth; (iv) signal-to-noise ratio (SNR); and (v) sensitivity roll-off in depth.

Several factors can influence these parameters. First, different kinds of samples may have different wavelength-dependent scattering and absorption properties. The amount of scattering and absorption determines the optical losses in the sample. Therefore, the choice of center wavelength $\lambda_{0}$ of an OCT system influences the imaging depth in a given sample. For example, in ophthalmology, imaging is commonly operated at a center wavelength of $800 \mathrm{~nm}$ where the water absorption is low [24]. When imaging skin, which exhibits much more light scattering than the eye, a longer center wavelength is preferred, such as $1.3 \mu \mathrm{m}$ or 
$1.7 \mu \mathrm{m}$ [25], as longer wavelengths show reduced scattering and therefore allow for larger imaging depths.

Secondly, the axial resolution of an FD-OCT system depends on the effective spectral bandwidth of the detected light. The effective spectral bandwidth depends on the spectral bandwidth of the light source, the wavelength response of all the optical elements along the light path and the wavelength response of the detector. If we assume a Gaussian shaped spectrum, the axial resolution $(\Delta z)$ in air is related to the full-width-at-half-maximum (FWHM) bandwidth $(\Delta \lambda)$ of the spectrum as [26]:

$$
\Delta z=\ln (2) \frac{2}{\pi} \frac{\lambda_{0}^{2}}{\Delta \lambda}
$$

where $\lambda_{0}$ is the center wavelength of the spectrum. It is shown that the larger the effective bandwidth, the better the axial resolution is for a given center wavelength. Note that this axial resolution is achievable only in a dispersion-free system. $\Delta z$ increases in case of a dispersion mismatch between the sample arm and the reference arm [27].

Thirdly, the maximum imaging depth $z_{\max }$ is determined by the spectral sampling interval ( $\delta k, k$ is the wavenumber). From Nyquist's sampling theorem, the maximum imaging depth is given by [21]

$$
\pm z_{\max }= \pm \frac{\pi}{2 \cdot \delta k}= \pm \frac{\lambda_{0}^{2}}{4 \cdot \delta \lambda} .
$$

It is shown that the smaller the spacing between the spectral channels the larger the maximum imaging depth is.

Fourthly, for maximum SNR, the optical losses in the system should be minimized. However, the exact influence of optical losses on the SNR is complicated. The same optical loss may influence the SNR differently under different system configurations and performance. A theoretical treatment of the influence of optical losses on the SNR is presented in Section 2.6. Typical SNR values for high-quality FD-OCT imaging are in the order of $100 \mathrm{~dB}[21]$.

Finally, sensitivity of an OCT system is defined as the ratio of the signal power generated by a perfectly reflecting mirror $(R=1)$ and that generated by $R_{\min } . R_{\min }$ is the weakest sample reflectance which yields a signal power equal to the noise power of the system [28]. The finite spectral resolution of an OCT system leads to reduced fringe visibility at higher fringe frequencies [29]. Thus, the amplitude of the signal from a perfectly reflecting mirror reduces at larger depth (higher fringe frequencies). Therefore, the sensitivity decreases as a function of depth. The magnitude of the roll-off $\Re_{\text {roll-off }}(z)$ in sensitivity is given by [30] 


$$
\Re_{\text {roll-off }}(z)=\left(\frac{\sin z^{\prime}}{z^{\prime}}\right) \cdot \exp \left|-\frac{\psi^{2}}{2 \ln 2} z^{\prime 2}\right|,
$$

where $z^{\prime}=(\pi / 2) \cdot\left(z / z_{\text {MAX }}\right)$ denotes the depth normalized to the maximum imaging depth $z_{\max }$ and $\psi=\delta \lambda_{r} / \delta \lambda$ denotes the ratio between the spectral resolution $\delta \lambda_{r}$ (FWHM) and the spectral sampling interval $\delta \lambda$. The sensitivity roll-off is commonly quantified as a $6 \mathrm{~dB}$ rolloff depth $z 6 \mathrm{~dB}$. It is defined as the one-sided depth at which the sensitivity falls off by a factor of $1 / 2$ or $6 \mathrm{~dB}$ in OCT SNR units [21] (the OCT SNR unit is introduced in Section 2.5)

$$
z_{6 d B}=\frac{2 \ln 2}{\delta k_{r}}=\frac{\ln 2}{\pi} \frac{\lambda_{0}^{2}}{\delta \lambda_{r}},
$$

where $\delta k_{r}$ is the spectral resolution in wavenumber. Note both Equation (1.2.3) and Equation (1.2.4) are only valid in case of a constant sample-detector coupling (independent of sample position). In practice, this is only approximate true within the focal depth of the beam. Outside the focal depth of the beam, the reduced coupling efficiency leads to a smaller OCT signal and thus effectively reduces the sensitivity. Therefore, the exact sensitivity roll-off also depends on the beam properties such as the focal size and its location relative to the sample.

The parameters discussed above are important for the imaging quality of a FD-OCT system. Therefore, they have to be taken into account when designing an FD-OCT chip. However, not all of these parameters are influenced by the chip design. Two of these parameters namely (iii) maximum imaging depth and (v) spectral induced sensitivity roll-off in depth are independent of the chip design. They only depend on the spectral resolution of the spectrometer (in case of SD-OCT) or swept laser and detector (in case of SS-OCT). The remaining three key parameters namely (i) the center wavelength, (ii) axial resolution and (iv) SNR determine different aspects of the chip design criteria. First, all the optical components on chip should be transparent at the OCT center wavelength, which is chosen based on the application. Second, for minimum axial resolution $(\Delta z)$, maximum FWHM bandwidth $(\Delta \lambda)$ of the detected spectrum is needed. Therefore, all the optical components need a working wavelength range as broad as possible. A wavelength dependent optical power efficiency (caused by the beam splitting ratio at the beam splitter and the optical losses of all the optical components) can effectively reduce the detected FWHM bandwidth $\Delta \lambda$. Thus, wavelength dependence in terms of optical efficiency of all the optical components needs to be minimized. Third, for maximum SNR, the optical losses in the chip should be minimized.

To summarize, the following design criteria are considered for the OCT-chip design. Requirements:

(1) Transparent at the intended OCT wavelength (such as $1.3 \mu \mathrm{m}$ ). 
Objectives:

(2) As broad as possible working wavelength range (such as a few hundreds of $\mathrm{nm}$ ).

(3) As small as possible overall optical losses in the working wavelength range.

(4) As small as possible wavelength dependence in terms of the beam splitting ratio at the beam splitter and the optical losses of all the optical components.

(5) Small or no dispersion between signal and reference arm

(6) Small overall dimensions of the system

The chip design requirements and objectives discussed above are taken into account when designing a FD-OCT chip. A more detailed discussion is to be found in Section 1.4.

\subsection{Literature survey of existing chip-based FD-OCT systems}

Recently, several chip-based FD-OCT systems have been demonstrated. They are briefly described below and their performances are summarized in Table 1.3.1. Akca et al. demonstrated a SD-OCT with an $2 \times 2$ splitter and an integrated spectrometer based on silicon oxynitride ( $\mathrm{SiON}$ ) waveguides, where the reference arm was not integrated on the chip [31]. Nguyen et al. demonstrated a SS-OCT system with a $\mathrm{Si}_{3} \mathrm{~N}_{4}$ waveguide-based interferometer and reference arm [32]. However, the length of their on-chip reference arm was not long enough to compensate the optical path introduced by a galvanometer scanner in the sample arm. Thus, the sample was translated to obtain B-scans. Yurtsever et al. demonstrated two different OCT systems [27,33]. Both systems have a sufficiently long on-chip reference arm to accommodate a galvanometer scanner in the sample arm to obtain B-scans. There are also studies that attempted to integrate on-chip tunable laser sources [34] and waveguide photodetectors [35] into chip-based SS-OCT systems. However, OCT imaging has not been demonstrated yet with these on-chip tunable lasers and photodetectors.

Even though a lot of progress has been made in the chip-based OCT studies mentioned above, there are still several common problems. Firstly, all studies are dual-arm systems, where there are separate reference and sample arms. In systems with an on-chip reference arm, the dispersion difference between the reference arm and the sample path needs to be compensated using methods that may reduce the attainable axial resolution $[27,33]$. The onchip reference arm has also relatively large dimensions, especially in low-contrast waveguide technology where the minimal bending radius is the limiting factor for miniaturization. Secondly, a common practical challenge in these chip-based systems is the design and fabrication of a broad-bandwidth 50/50 coupler to be used in the interferometer, see e.g. [36]. Directional couplers are wavelength-dependent devices of which the coupling ratio is strongly dependent on fabrication accuracy, as reported in Ref. [32]. Any deviation from a $50 / 50$ splitting ratio decreases the efficiency of the OCT system. Thirdly, in all of these chip- 
based OCT studies, external lenses are used for the optical chip-to-sample coupling. These external elements can be much larger than the chip itself.

In Section 1.4, we present our chip-based OCT system design. We explain how this design may overcome the limitations of current chip-based OCT systems.

Table 1.3.1. Overview of the performance of reported chip-based OCT systems.

\begin{tabular}{|c|c|c|c|c|}
\hline Ref. & [31] & {$[32]$} & [27] & [33] \\
\hline Type of OCT & SD-OCT & SS-OCT & SS-OCT & SD-OCT \\
\hline $\begin{array}{l}\text { Waveguide } \\
\text { technology }\end{array}$ & $\mathrm{SiON}$ & $\mathrm{Si}_{3} \mathrm{~N}_{4}{ }^{*}$ & $\begin{array}{c}\text { Silicon-on- } \\
\text { insulator (SOI) }\end{array}$ & $\mathrm{Si}_{3} \mathrm{~N}_{4}{ }^{*}$ \\
\hline $\begin{array}{c}\text { Center wavelength } \\
\lambda_{0}(\mathrm{~nm})\end{array}$ & 1320 & 1312 & 1312 & 1320 \\
\hline $\begin{array}{l}\text { Axial resolution } \\
\qquad \Delta z(\mu \mathrm{m})\end{array}$ & $7.5^{* *}$ & $12.7 \pm 0.5^{* * *}$ & $25.5^{* * *}$ & $14^{* * * *}$ \\
\hline $\begin{array}{l}\text { Maximum imaging } \\
\text { depth } z \max (\mathrm{mm})\end{array}$ & $1.4^{* *}$ & $5.09^{* * *}$ & $5.09^{* * * *}$ & $3.4^{* * *}$ \\
\hline $\begin{array}{c}\text { Sensitivity } \\
(\mathrm{dB})\end{array}$ & $74^{* * * * *}$ & 80 & 62 & 65 \\
\hline $\begin{array}{c}6 \mathrm{~dB} \text { roll-off depth } \\
z \text { 6dB }(\mathrm{mm})\end{array}$ & $\sim 0.7^{* * * * * *}$ & $\sim 1.7^{* * * * * *}$ & 1 & 1 \\
\hline Chip size & $0.36 \mathrm{~cm}^{3}$ & $0.4 \times 1.8 \mathrm{~cm}^{2}$ & $0.075 \times 0.5 \mathrm{~cm}^{2}$ & $1.0 \times 3.3 \mathrm{~cm}^{2}$ \\
\hline
\end{tabular}

\subsection{Overview of the FD-OCT chip design}

\subsubsection{Components overview for on-chip functionality}

The designed chip needs to have several functions in order to have a working FD-OCT system. In this section, we provide a discussion about these required functions and the integrated optical components that can be used to realize these functions.

First, the light has to be guided on the chip, therefore, a suitable waveguide technology is needed. As can be seen from Table 1.3.1, many different waveguide materials are possible for OCT applications. There is no fundamental restriction to choose one or another as long 
as the material is transparent in the wavelength range of interest (defined by the application). Of course, the propagation loss of these waveguides does influence the OCT SNR. Thus, low loss waveguide technology is preferred. The applied waveguide technology and the waveguide design have no influence on the system design principle. Therefore we will not discuss details on the waveguide here, but leave this discussion for Chapter 3.

Second, an on-chip beam splitter is needed. There are four possible components to realize this on-chip beam splitter: (i) directional coupler; (ii) multimode interference (MMI) coupler; (iii) Y junction; (iv) circulator. Among these options, a circulator proves the best power efficiency (close to $100 \%$ ), thus, it is widely used in fiber based systems [30, 37, 38]. However, up to now, the on-chip circulator is very challenging to design and fabricate, especially for a broad wavelength range with low loss [39]. Directional coupler, MMI and Y junction all have maximum power efficiency of $25 \%$ (50/50 power splitting without loss) calculated from the light source (through the sample reflection) to the detector. In practice, the MMI and Y junction may have additional losses in the order of a few percent. Based on the analysis given in Section 2.6, a few percent additional loss (such as 10\%) in these components only reduces the SNR by maximum $\sim 2 \mathrm{~dB}$. As can be seen from Table 1.3.1, $2 \mathrm{~dB}$ reductions to the SNR (or the sensitivity) is small compared to the total sensitivity. Thus the loss difference between a directional coupler, an MMI and a Y junction is not a main concern. Y junctions are intrinsically wavelength independent. Thus they better satisfy two of the design objectives (broad working wavelength range and small wavelength dependence) compared with the directional coupler and the MMI. Therefore, the Y junction is chosen to realize the on-chip beam splitter in our design.

Third, an on-chip reference arm and reference reflector is needed. There are two types of reference arm approaches, namely dual arm and common-path. The dual arm OCT has a separate sample arm and reference arm. Loop mirrors are used in dual arm OCT to realize the reference arm and reference reflector [27, 32, 33]. The common-path OCT utilizes a partial reflection in the sample arm as the reference reflection. Thus, the sample arm also serves as reference arm. In this case the end facet of the waveguide can act as the reference reflector. Both approaches have advantages and disadvantages. The advantage of the loop mirror approach is that the sample can be far away (in the order of few tens of centimeters) from the chip. The sample-chip distance can be designed freely by varying the optical length of the loop mirror. The disadvantages are the relatively large feature size (compare with the common-path approach) of the loop mirror and dispersion mismatch (between the loop mirror and optical path outside chip) which induces axial resolution degradation [27]. The main advantage of the common-path approach is that it enables canceling out many unwanted effects (such as dispersion and polarization dependency) due to the shared sample and reference arm. Another advantage is the minimized chip area by eliminating the reference arm. The disadvantage is that the sample has to be placed close (within the maximum depth range of the OCT system) to the chip. Fortunately, in our application (imaging of skin) the 
problem of a small sample-chip distance is less apparent. Thus, a common-path approach with the waveguide end facet as the reference reflector is chosen for our chip design.

Fourth, integrated and efficient chip-sample coupling is needed. Small waveguide mode sizes (on the order of 1-3 $\mu \mathrm{m}$ ) lead to strong divergence, on the order of tens of degrees, of the out-coupled beam. This strong diverging beam not only results in poor lateral resolution but also in low optical coupling efficiency from the back reflected light from the sample to the waveguide. Thus, external lenses are used for the optical coupling between chip and sample in current chip-based OCT systems as mentioned in Section 1.3. We would like to have an on-chip solution to this coupling problem in this thesis. To our knowledge, there are two types of technologies that may provide integrated solutions to focus or collimate the light from a waveguide to the sample and collect the reflected light into the waveguide again. The first option is a grating based method such as focusing grating couplers, which couples the light out of the chip plane [40], or an end facet plasmonic grating [41]. However, gratings are inherently wavelength dependent which may limit the working wavelength range of an OCT [27]. The second option is the use of micrometer sizes lenses. A lens yields in general a broad working wavelength range which is a major advantage compared with grating couplers. Therefore, we will develop a microlens structure suitable for on-chip OCT applications.

In the next section we will combine the design choices given above providing an overall system layout of the OCT chip.

\subsubsection{Chip-based FD-OCT system layout}

Fig.1.4.1 shows the basic design of our FD-OCT system. The light emitted from the light source is coupled to the chip through a fiber array unit (FAU). This light propagates through the Y-junction with a 50\% efficiency (more details can be found in Section 3.4). A 90 degree bend is used to redirect the guided light from the unguided stray light due to the imperfect light coupling between the input fiber and the chip. At the end facet of the waveguide, part of the light is reflected back into the waveguide due to the Fresnel reflection (the facet acts as a reference plane). The remaining light exits from the facet and is focused onto the sample with a micron sized lens. Part of the back reflected light from the sample is coupled into the waveguide by the same lens and interferes with the light that is reflected by the end facet. This interference signal propagates through the $\mathrm{Y}$-junction where $50 \%$ goes into the detection branch which is coupled to a detector through the FAU.

Our approach addresses the problems as discussed in Section 1.3 as follows. Firstly, by using a common-path OCT system we avoid the need of a separate reference arm. On our chip we exploit the back reflection from the end facet of the waveguide to act as the reference, thus preventing the need for a separate reference reflection. This solution not only saves space on the chip, but also eliminates the decrease of axial resolution caused by dispersion [27]. 
Secondly, the three ports (see figure 1.4.1) of the chip are connected by a symmetric Y junction.

Thirdly, a directly integrated micro-ball lens (diameter of $\sim 100 \mu \mathrm{m}$ ) [42] is positioned at a short distance from the waveguide facet for efficient coupling of the light between the chip and the sample. This micro-ball lens is the key component enabling the common-path configuration. Firstly, the lens significantly reduces the divergence angle of the light exiting from the waveguide, thus improving the lateral resolution and the chip-sample coupling compared to the case without a lens. Secondly, a typical FD-OCT has a maximum imaging depth $z_{\max }$ in the order of few millimeters (measured from the end facet of the waveguide). Therefore, the sample should be within this distance from the end-facet of the waveguide. Clearly this is not feasible with a large external lens. The use of an integrated micro-ball lens which occupies only the first $\sim 0.2 \mathrm{~mm}$ (see Section 4.4 ), leaves sufficient room for the sample.

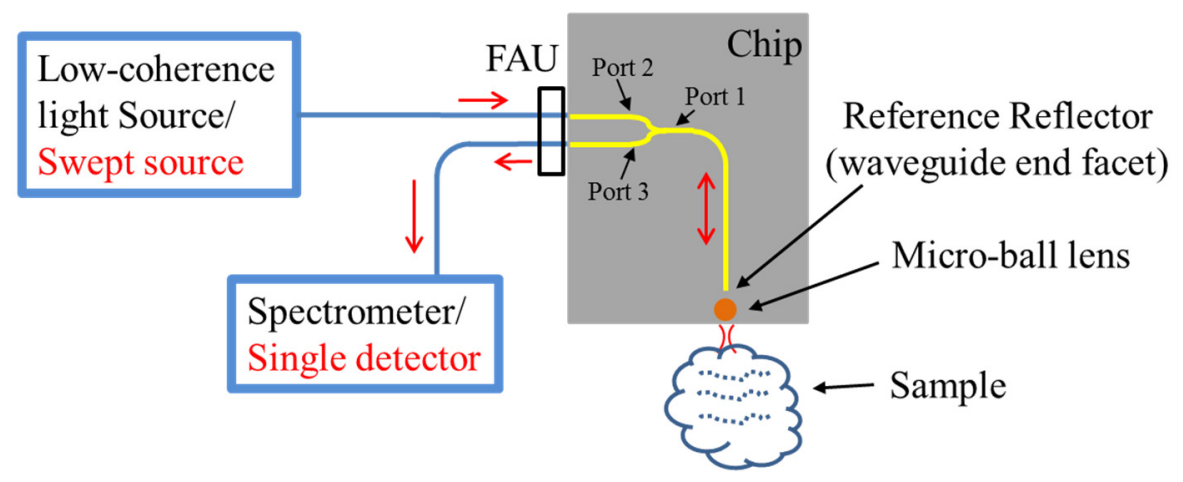

Fig. 1.4.1. Schematic of the partially-integrated FD-OCT system with a common-path configuration and an integrated micro-ball lens. Light emitted by the source coupled to the chip through a fiber array unit (FAU). The blue lines indicate optical fibers and the yellow lines represent the waveguides on the chip. The same chip can be used in both SD-OCT (combination of a low-coherence light source and a spectrometer) and SS-OCT (combination of a swept source and a single detector) systems.

In our system layout, there is no space for a beam scanning device in between the chip and the sample. However, since the micro-ball lens is integrated and the optical chip is a small low-mass device, in an alternative implementation, the fiber-connected chip can be mounted directly onto a scanner to obtain two- or three-dimensional images. A galvanometric $\mathrm{x}-\mathrm{y}$ scanner needs to scan only small angles to obtain relatively wide areas. In a chip scanning approach, the chip probably has to scan/move over much larger distances to cover the same area, which may be a limiting factor for the ultimate scan speed.

The detailed design of all on chip components are discussed in Chapters 3-4. 


\subsection{Outline of the thesis}

In this thesis, we will introduce a detailed mathematical model of the FD-OCT system in Chapter 2. It is useful to understand the working principle, signal, noise and multiple reference reflections induced effects in an FD-OCT before designing the individual components. Chapter 3 is focused on the design, fabrication and characterization of the waveguide components. Chapter 4 is focused on the design, fabrication and characterization of the micro-ball lens. A SS-OCT setup is introduced in Chapter 5, which also details how we characterize the performance of our OCT design. The detailed studies include the data analysis method; OCT signal and noise analysis; performance comparison of the chip-based system with a lens, the same system without a lens and a fiber-based system. In addition, we provide a method to suppress ghost images that arise from the inherent multiple reference reflections in the OCT-chip. The small feature size of our common-path OCT design is attractive for parallel OCT construction. In Chapter 6 we explore the possible designs for single layer and double layer parallel OCT design. Finally, in Chapter 7, we discuss the main conclusions of this thesis. 


\section{References}

1. Huang, D., E.A. Swanson, C.P. Lin, J.S. Schuman, W.G. Stinson, W. Chang, M.R. Hee, T. Flotte, K. Gregory, C.A. Puliafito, and J.G. Fujimoto, Optical Coherence Tomography. Science, 1991. 254(5035): p. 1178-1181.

2. Dhawan, A.P., B. D'Alessandro, and X. Fu, Optical imaging modalities for biomedical applications. Biomedical Engineering, IEEE Reviews in, 2010. 3: p. 69-92.

3. Akca, B.I., Spectral-domain optical coherence tomography on a silicon chip. 2012.

4. Welzel, J., E. Lankenau, R. Birngruber, and R. Engelhardt, Optical coherence tomography of the human skin. Journal of the American Academy of Dermatology, 1997. 37(6): p. 958-963.

5. Fried, D., J. Xie, S. Shafi, J.D. Featherstone, T.M. Breunig, and C. Le, Imaging caries lesions and lesion progression with polarization sensitive optical coherence tomography. Journal of biomedical optics, 2002. 7(4): p. 618-627.

6. Costa, R.A., M. Skaf, L.A.S. Melo, D. Calucci, J.A. Cardillo, J.C. Castro, D. Huang, and M. Wojtkowski, Retinal assessment using optical coherence tomography. Progress in Retinal and Eye Research, 2006. 25(3): p. 325-353.

7. Zysk, A.M., F.T. Nguyen, A.L. Oldenburg, D.L. Marks, and S.A. Boppart, Optical coherence tomography: a review of clinical development from bench to bedside. Journal of Biomedical Optics, 2007. 12(5): p. 051403-051403-21.

8. Gupta, V., A. Gupta, and M.R. Dogra, Atlas of Optical Coherence Tomography of Macular Diseases. 2004: CRC Press.

9. Hee, M.R., C.A. Puliafito, C. Wong, J.S. Duker, E. Reichel, B. Rutledge, J.S. Schuman, E.A. Swanson, and J.G. Fujimoto, Quantitative assessment of macular edema with optical coherence tomography. Archives of ophthalmology, 1995. 113(8): p. 1019-1029.

10. Brezinski, M.E., G.J. Tearney, B.E. Bouma, S.A. Boppart, M.R. Hee, E.A. Swanson, J.F. Southern, and J.G. Fujimoto, Imaging of coronary artery microstructure (in vitro) with optical coherence tomography. American Journal of Cardiology, 1996. 77(1): p. 92-93.

11. Tearney, G.J., M.E. Brezinski, J.F. Southern, B.E. Bouma, S.A. Boppart, and J.G. Fujimoto, Optical biopsy in human gastrointestinal tissue using optical coherence tomography. American Journal of Gastroenterology, 1997. 92(10): p. 1800-1804.

12. Pitris, C., A. Goodman, S.A. Boppart, J.J. Libus, J.G. Fujimoto, and M.E. Brezinski, High-resolution imaging of gynecologic neoplasms using optical coherence tomography. Obstetrics and Gynecology, 1999. 93(1): p. 135-139.

13. Jung, W.G., J. Zhang, J.R. Chung, P. Wilder-Smith, M. Brenner, J.S. Nelson, and Z.P. Chen, Advances in oral cancer detection using optical coherence tomography. Ieee Journal of Selected Topics in Quantum Electronics, 2005. 11(4): p. 811-817.

14. Pitris, C., M.E. Brezinski, B.E. Bouma, G.J. Tearney, J.F. Southern, and J.G. Fujimoto, High resolution imaging of the upper respiratory tract with optical coherence tomography - A feasibility study. American Journal of Respiratory and Critical Care Medicine, 1998. 157(5): p. 1640-1644.

15. Mueller-Lisse, U.L., M. Bader, M. Bauer, E. Engelram, Y. Hocaoglu, M. Püls, O.A. Meissner, G. Babaryka, R. Sroka, and C.G. Stief, Optical coherence tomography of the upper urinary tract: Review of initial experience ex vivo and in vivo. Medical Laser Application, 2010. 25(1): p. 44-52.

16. Dufour, M., G. Lamouche, B. Gauthier, C. Padioleau, and J.-P. Monchalin. Inspection of hard-to-reach industrial parts using small-diameter probes. in Proceedings of the SPIE. 2006.

17. Song, G. and K. Harding. OCT for industrial applications. 2012.

18. Walecki, W.J., K. Lai, A. Pravdivtsev, V. Souchkov, P. Van, T. Azfar, T. Wong, S.H. Lau, and A. Koo. Low-coherence interferometric absolute distance gauge for study of MEMS structures. 2005. 
19. Walecki, W.J., K. Lai, V. Souchkov, P. Van, S.H. Lau, and A. Koo, Novel noncontact thickness metrology for backend manufacturing of wide bandgap light emitting devices. E-MRS 2004 Fall Meeting Symposia C and F, 2005. 2(3): p. 984-989.

20. Stifter, D., Beyond biomedicine: a review of alternative applications and developments for optical coherence tomography. Applied Physics B-Lasers and Optics, 2007. 88(3): p. 337-357.

21. Drexler, W. and J.G. Fujimoto, Optical Coherence Tomography: Technology and Applications. 2008: Springer.

22. Fercher, A.F., Optical coherence tomography - development, principles, applications. Zeitschrift Fur Medizinische Physik, 2010. 20(4): p. 251-276.

23. Choma, M.A., M.V. Sarunic, C.H. Yang, and J.A. Izatt, Sensitivity advantage of swept source and Fourier domain optical coherence tomography. Optics Express, 2003. 11(18): p. 2183-2189.

24. Podoleanu, A.G., Optical coherence tomography. Journal of Microscopy, 2012. 247(3): p. 209-219.

25. Sharma, U., E.W. Chang, and S.H. Yun, Long-wavelength optical coherence tomography at $1.7 \mathrm{mu}$ for enhanced imaging depth. Optics Express, 2008. 16(24): p. 19712-19723.

26. Swanson, E.A., D. Huang, M.R. Hee, J.G. Fujimoto, C.P. Lin, and C.A. Puliafito, High-Speed Optical Coherence Domain Reflectometry. Optics Letters, 1992. 17(2): p. 151-153.

27. Yurtsever, G., N. Weiss, J. Kalkman, T.G. van Leeuwen, and R. Baets, Ultra-compact silicon photonic integrated interferometer for swept-source optical coherence tomography. Optics Letters, 2014. 39(17): p. 5228-5231.

28. Fercher, A.F., W. Drexler, C.K. Hitzenberger, and T. Lasser, Optical coherence tomography principles and applications. Reports on Progress in Physics, 2003. 66(2): p. 239-303.

29. Dorrer, C., N. Belabas, J.-P. Likforman, and M. Joffre, Spectral resolution and sampling issues in Fourier-transform spectral interferometry. JOSA B, 2000. 17(10): p. 1795-1802.

30. Yun, S., G. Tearney, B. Bouma, B. Park, and J. de Boer, High-speed spectral-domain optical coherence tomography at $1.3 \mu \mathrm{m}$ wavelength. Optics Express, 2003. 11(26): p. 3598-3604.

31. Akca, B.I., B. Považay, A. Alex, K. Wörhoff, R.M. de Ridder, W. Drexler, and M. Pollnau, Miniature spectrometer and beam splitter for an optical coherence tomography on a silicon chip. Optics Express, 2013. 21(14): p. 16648-16656.

32. Nguyen, V.D., N. Weiss, W. Beeker, M. Hoekman, A. Leinse, R.G. Heideman, T.G. van Leeuwen, and J. Kalkman, Integrated-optics-based swept-source optical coherence tomography. Optics Letters, 2012. 37(23): p. 4820-4822.

33. Yurtsever, G., B. Považay, A. Alex, B. Zabihian, W. Drexler, and R. Baets, Photonic integrated MachZehnder interferometer with an on-chip reference arm for optical coherence tomography. Biomedical Optics Express, 2014. 5(4): p. 1050-1061.

34. Tilma, B.W., Y.Q. Jiao, J. Kotani, B. Smalbrugge, H.P.M.M. Ambrosius, P.J. Thijs, X.J.M. Leijtens, R. Notzel, M.K. Smit, and E.A.J.M. Bente, Integrated Tunable Quantum-Dot Laser for Optical Coherence Tomography in the 1.7 mu $\mathrm{m}$ Wavelength Region. Ieee Journal of Quantum Electronics, 2012. 48(2): p. 87-98.

35. Jiao, Y.Q., B.W. Tilma, J. Kotani, R. Notzel, M.K. Smit, S.L. He, and E.A.J.M. Bente, InAs/InP(100) quantum dot waveguide photodetectors for swept-source optical coherence tomography around $1.7 \mathrm{mu}$ $m$. Optics Express, 2012. 20(4): p. 3675-3692.

36. Akca, B.I., C.R. Doerr, G. Sengo, K. Worhoff, M. Pollnau, and R.M. de Ridder, Broad-spectral-range synchronized flat-top arrayed-waveguide grating applied in a 225-channel cascaded spectrometer. Optics Express, 2012. 20(16): p. 18313-18318.

37. Zhao, M., Y. Huang, and J.U. Kang, Sapphire ball lens-based fiber probe for common-path optical coherence tomography and its applications in corneal and retinal imaging. Optics Letters, 2012. 37(23): p. 4835-4837.

38. Weiss, N., T.G. van Leeuwen, and J. Kalkman, Localized measurement of longitudinal and transverse flow velocities in colloidal suspensions using optical coherence tomography. Physical Review E, 2013. 88(4): p. 042312. 
39. Pintus, P., F. Di Pasquale, and J.E. Bowers, Integrated TE and TM optical circulators on ultra-low-loss silicon nitride platform. Optics Express, 2013. 21(4): p. 5041-5052.

40. Van Laere, F., T. Claes, J. Schrauwen, S. Scheerlinck, W. Bogaerts, D. Taillaert, L. O'Faolain, D. Van Thourhout, and R. Baets, Compact focusing grating couplers for silicon-on-insulator integrated circuits. Ieee Photonics Technology Letters, 2007. 19(21-24): p. 1919-1921.

41. Gmachl, C., Plasmonics - A sharper approach. Nature Photonics, 2008. 2(9): p. 524-525.

42. Chang, L., M. Dijkstra, N. Ismail, M. Pollnau, R.M. de Ridder, K. Wörhoff, V. Subramaniam, and J.S. Kanger, Waveguide-coupled micro-ball lens array suitable for mass fabrication. Optics Express, 2015. 23(17): p. 22414-22423. 


\section{Chapter 2}

\section{Mathematical model of a FD-OCT}

\subsection{Introduction}

The aim of this chapter is to present a generalized model of Fourier domain OCT (FD-OCT) which includes the optical path transmission factors and multiple reference reflectors. Izatt and Choma have presented a detailed theory of FD-OCT based on a free-space Michelson interferometer model (50/50 beam splitter and lossless optical paths) with a single reference reflector in Section 2.3 of Ref. [1]. In Section 2.2 of this chapter, we start with their Michelson interferometer model and generalize the transmission factors of all paths (non 50/50 beam splitter and lossy optical paths) in the system. By introducing those factors, this model can be applied to a fiber- or a chip-based system which may have a considerable amount of loss compared with an ideal system. The optimization of an FD-OCT system in terms of signal strength and shot noise limited signal-to-noise ratio (SNR) can then be performed quantitatively. This generalized model may also be used for an FD-OCT system with optical amplification by simply applying a transmission factor larger than one. This generalized model can also be used for other interferometer based FD-OCT system, such as a commonpath OCT, since all the transmission factors have been defined separately.

In Section 2.3, we further generalize the model to a system with multiple reference reflectors. Reflections from optical element interfaces may act as additional references. Ghost OCT signals will be measured in the case that these reflections are very close (within the FD-OCT maximum depth range) to the intended reference reflection. We show mathematically that the sample-specific information can be recovered from the distorted measured signal by deconvolution. This solution will be used in our on-chip common-path FD-OCT system which will be introduced in Chapter 5.

In Section 2.4, we discuss several kinds of noise present in an FD-OCT system, including readout noise, shot noise, relative intensity noise and the noise introduced by the

Parts of this chapter is accepted by Optics Express as: Chang, L., N. Weiss, T.G. van Leeuwen, M. Pollnau, R.M. de Ridder, K. Wörhoff, V. Subramaniam, and J.S. Kanger, Chip based common-path optical coherence tomography system with an on-chip microlens and multi-reference suppression algorithm. 
Fourier transform.

In Section 2.5, we first clarify the definition of the SNR in an A-scan, which we use throughout this thesis. We then discuss the signal and noise propagation during the Fourier transform and provide a formula for the shot noise limited SNR using the generalized transmission factors introduced in Section 2.2.

In Section 2.6, we discuss the influence on OCT SNR by the optical losses in different components. This is used as a guideline during the design optimization of the chip components.

\subsection{FD-OCT signal with single reference reflector}

A mathematical description of an FD-OCT signal is discussed in this Section. This description is similar to the one presented Izatt and Choma in Section 2.3 of Ref. [1], but with a more general set of parameters. Those generalized parameters are needed to accurately describe our on-chip OCT system.

For a typical FD-OCT setup, as shown in Fig.2.2.1, the light source provides a polychromatic plane wave of which the electric field is expressed in complex form as

$$
E_{i}=s(k) e^{i(k z-\omega t)},
$$

where $\omega$ is the angular frequency, $k$ is the wavenumber, $z$ is the distance from the source along the optical axis, and $s(k)$ is the amplitude spectral density of the electric field. The field transmission coefficient from the light source to the reference reflector is denoted by $t_{L R}$ and that from the reference reflector to the detector is given by $t_{R D}$. Furthermore, the field reflection coefficient of the reference reflector is described by $r_{R}$. Thus, in the absence of the sample arm the field incident on the detector after returning from the reference is:

$$
E_{R}=E_{i}\left(z=z_{L}\right) t_{L R} t_{R D} r_{R} e^{i k\left(2 z_{R}+z_{D}\right)},
$$

where $z_{L}, z_{R}$ and $z_{D}$ are the optical distances from the light source to the beam splitter, from the beam splitter to the reference reflector and from the beam splitter to the detector respectively. Next, we define the field transmission coefficient from the light source to the sample as $t_{L S}$ and from the sample to the detector as $t_{S D}$. If the sample is modeled as a set of $N$ discrete partial reflectors with respective field reflection coefficients $r_{S n}$, which incorporate the depth-dependent absorption and scattering losses in the sample, the field incident on the detector after returning from the sample, in the absence of the reference arm, is: 


$$
E_{S}=E_{i}\left(z=z_{L}\right) t_{L S} t_{S D} e^{i z_{D}} \sum_{n=1}^{N}\left(r_{S n} e^{2 i k z_{S n}}\right),
$$

where $z_{S n}$ is the optical distance from the beam splitter to the $n^{\text {th }}$ reflector in the sample.

\section{Reference Reflector}

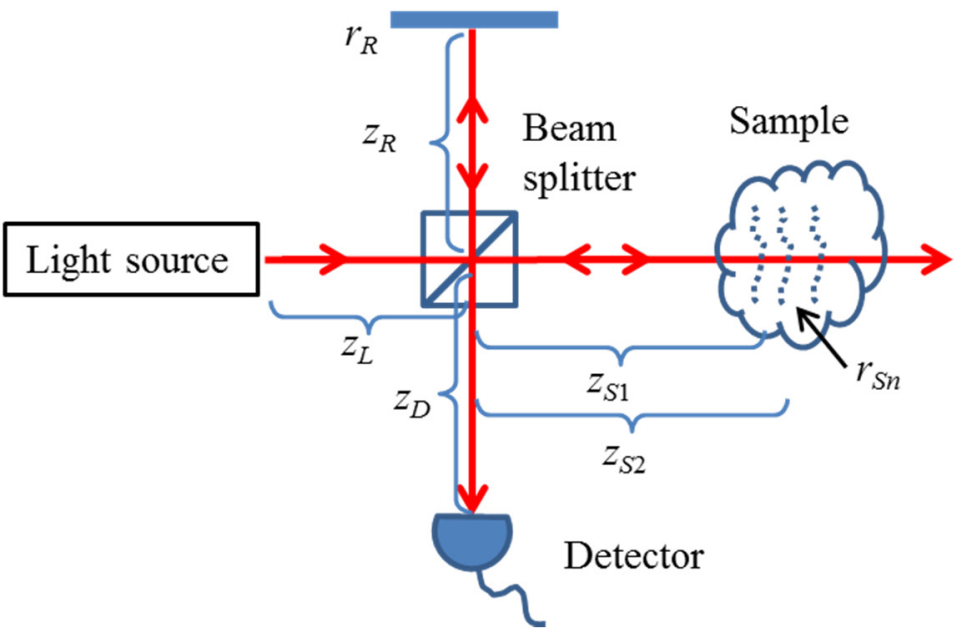

Fig. 2.2.1. Schematic of a free space Michelson interferometer based OCT system. All the symbols are explained in detail in the main text.

In the case that both arms are present, the waves returning from reference and sample interfere at the detector. The photocurrent generated by the detector is proportional to the square of the sum of the fields incident upon it, as given by

$$
I_{D}(k)=\rho(k)\left\langle c \varepsilon_{0} A\left|E_{R}+E_{S}\right|^{2}\right\rangle .
$$

Here $\rho(k)$ is the responsivity of the detector as a function of wavenumber (units Amperes/Watt), the angular brackets denote the (time) average over the response time of the detector, $c$ is the speed of light, $\varepsilon_{0}$ is the vacuum permittivity and $A$ is the area of the light beam (assume the detection area is larger than the beam). Choosing $z_{L}=-z_{D}$ for convenience (mathematically, the values of $z_{L}$ and $z_{D}$ do not influence the further calculation. Physically, the optical path from the light source to the beam splitter and from the beam splitter to the detector are the common-path for both sample and reference arms, thus the light interference is independent of the location of the light source and detector), the current spectral density is calculated as

$$
I_{D}(k)=\rho(k)\left\langle c \varepsilon_{0} A\left|s(k) t_{L R} t_{R D} r_{R} e^{i\left(2 k z_{R}-\omega t\right)}+s(k) t_{L S} t_{S D} \sum_{n=1}^{N}\left[r_{S n} e^{i\left(2 k z_{S n}-\omega t\right)}\right]\right|^{2}\right\rangle .
$$


As the period of the source optical wave is much smaller than the detector response time, and the system is assumed to be stationary, this leaves the temporally invariant terms

$$
I_{D}(k)=\rho(k) S(k)\left[T_{L R} T_{R D} R_{R}+T_{L S} T_{S D}\left(R_{S 1}+R_{S 2}+\ldots\right)\right]
$$

'Direct current (DC) Terms'

$$
+\rho(k) S(k) \sqrt{T_{L R} T_{R D} T_{L S} T_{S D}} \sum_{n=1}^{N}\left\{\sqrt{R_{R} R_{S n}}\left[e^{i 2 k\left(z_{S_{n}}-z_{R}\right)}+e^{-i 2 k\left(z_{s_{n}}-z_{R}\right)}\right]\right\}
$$

'Cross-correlationTerms'

$$
+\frac{1}{2} \rho(k) S(k) T_{L S} T_{S D} \sum_{\substack{n=1 \\ n \neq m}}^{N} \sum_{m=1}^{N}\left\{\sqrt{R_{S n} R_{S m}}\left[e^{i 2 k\left(z_{S n}-z_{S m}\right)}+e^{-i 2 k\left(z_{S n}-z_{S m}\right)}\right]\right\}
$$

'Auto-correlation Terms'.

Here,

$$
S(k)=\frac{1}{2} c \varepsilon_{0} A|s(k)|^{2}
$$

is the power spectral density of the light source, and

$$
T_{L R}=\left|t_{L R}\right|^{2}, T_{R D}=\left|t_{R D}\right|^{2}, T_{L S}=\left|t_{L S}\right|^{2}, T_{S D}=\left|t_{S D}\right|^{2}, \quad R_{R}=\left|r_{R}\right|^{2} \text { and } R_{S n}=\left|r_{S n}\right|^{2}
$$

are the optical power transmittance and reflectance. The factor $1 / 2$ in the 'Auto-correlation Terms' is a result of the double counting of $n=\mathrm{a}$ and $m=\mathrm{b}$ with the identical contributions of $n=\mathrm{b}$ and $m=\mathrm{a}$.

Equation (2.2.6) is similar to Equation (2.9) in [1]. There are three distinct components which are well described by Izatt and Choma as follows [1]:

1. A 'DC' component which is a pathlength-independent offset to the detector current. This is the largest component of the detector current if the detected reference power dominates the sample power.

2. A 'cross-correlation' component for each sample reflector, which depends upon both light source wavenumber and the pathlength difference between the reference arm and sample reflectors. This is the desired component for OCT imaging. Since these components are proportional to the square root of the sample reflectances (on the order of $\sim 10^{-4}$ to $10^{-5}$ for biological tissues), they are typically smaller than the DC component.

3. 'Autocorrelation' terms representing interference occurring between the different sample reflectors. Since the autocorrelation terms depend linearly upon the power reflectance of the sample reflectors, a primary tool for decreasing autocorrelation artifacts is selection of the proper reference reflectivity so that the autocorrelation terms are small compared to the DC and interferometric terms. 
(a)

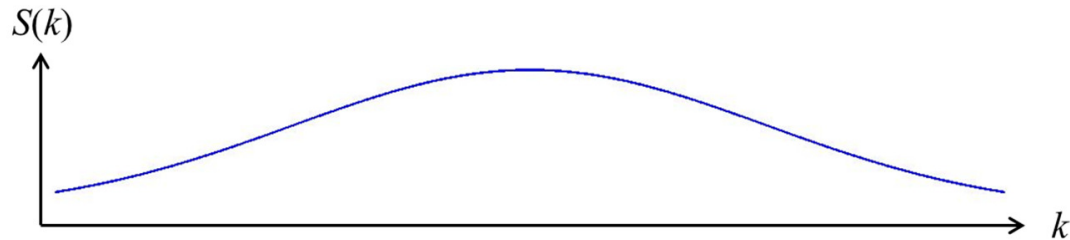

(b)

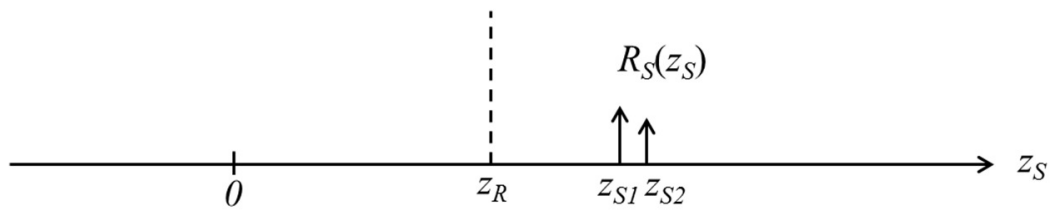

$I_{D}(k)$

(c)

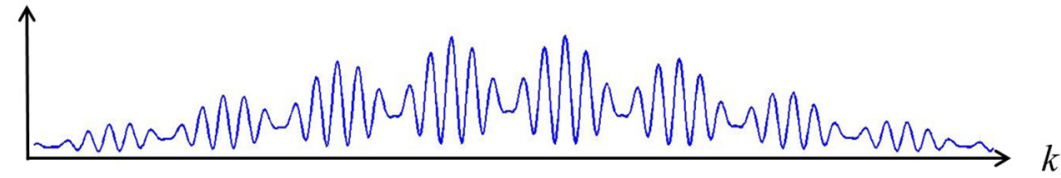

(d)

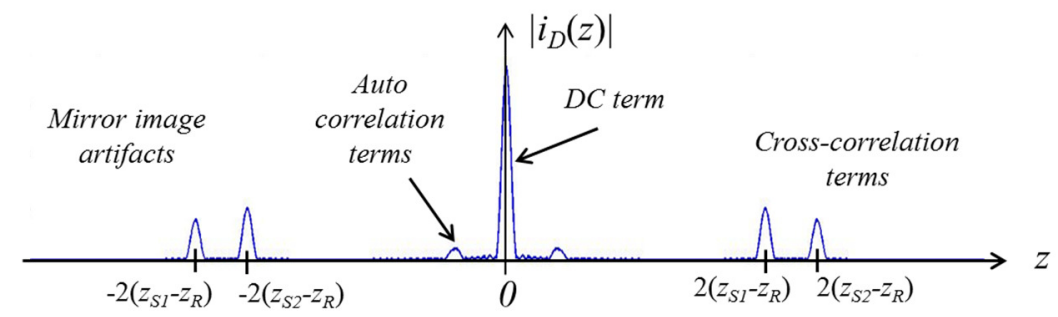

Fig. 2.2.2. Illustration of an FD-OCT A-scan signal construction. (a) The light source power spectral density with a Gaussian shape. (b) Discrete sample power reflectance $R_{S}\left(\mathrm{z}_{S}\right)=\sum_{n=1}^{N} R_{S n} \delta\left(z_{S}-z_{S n}\right)$.

(c) The detector current $I_{D}(k)$. (d) The A-scan result with a non-rescaled z axis.

The Fourier transform $i_{D}(z)$ of the measured $I_{D}(k)$ is an A-scan result from an FD-OCT. Note, the $z$ in $i_{D}(z)$ is the round trip optical path length difference between the reference and sample. In typical OCT data processing the A-scans are shown with a rescaled axis of $z / 2$ to shown the single trip optical path length difference which is corresponding to the depth in the sample. The axial resolution $\Delta z$ and maximum imaging depth $z$ max discussed in Section 1.2.2 refer to the rescaled $\mathrm{z}$ axis. In order to have less confusion in the mathematical description, we keep using the non-rescaled $\mathrm{z}$ axis in this chapter and use the commonly used rescaled $\mathrm{z}$ axis for the experimental results in Chapter 5. An example is shown in the Fig. 2.2.2. In this example, the light source has a Gaussian shape power spectral density $S(k)$ as shown in Fig. 2.2.2(a). The discrete sample power reflectance has a function 
of $R_{S}\left(\mathrm{z}_{S}\right)=\sum_{n=1}^{N} R_{S n} \delta\left(z_{S}-z_{S n}\right)$, as shown with solid arrows in Fig. 2.2.2(b), where $\delta()$ is the Kronecker delta function. The dashed line at $\mathrm{z}_{S}=z_{R}$ represents the reference reflector. The current spectral density $I_{D}(k)$ is shown in Fig. 2.2.2(c), which is a mixture of DC, crosscorrelation and autocorrelation terms. An A-scan result with a non-rescaled z axis, $\left|i_{D}(\mathrm{z})\right|$, is shown in Fig. 2.2.2(d), which is the Fourier transform of $I_{D}(k)$.

\subsection{FD-OCT signal with multiple reference reflectors}

Unwanted reflections in a FD-OCT system act as additional references. They produce artefacts (ghost images) when these reflections are close (less than the maximum depth range of the FD-OCT system) to the intended reference reflection. A mathematical description of eliminating these artefacts in a multiple references FD-OCT is introduced in this section. The experimental demonstration is in Chapter 5.

We further generalize the mathematical description given above by replacing the single reference reflector (see Equation 2.2.2 and Fig. 2.2.1) by $J$ discrete reflectors with effective reflection coefficients $r_{R j}$ (defined similar to the assumed discrete 'sample reflectors' $r_{S n}$ ). Note, $t_{L R}$ and $t_{R D}$ describes the transmission up to the first reference plane, and that additional transmission losses are artificially incorporated in the $r_{R j}$. Thus, the field incident on the detector after returning from the reference in the absence of the sample arm is:

$$
E_{R}=E_{i}\left(z=z_{L}\right) t_{L R} t_{R D} e^{i k z_{D}} \sum_{j=1}^{J}\left(r_{R j} e^{2 i k z_{R j}}\right),
$$

where $z_{R j}$ is the optical distance from the beam splitter to the $j^{\text {th }}$ reference reflector. Multiple reflections between these reflectors are considered as separate reflectors at different (longer) optical distances. The temporally invariant photocurrent generated by the detector is calculated in the same way as in Section 2.2 and results in 


$$
\begin{aligned}
I_{D}(k)= & \rho(k) S(k)\left[T_{L R} T_{R D}\left(R_{R 1}+R_{R 2}+\ldots\right)+T_{L S} T_{S D}\left(R_{S 1}+R_{S 2}+\ldots\right)\right] \\
& \text { 'DC Terms' } \\
+ & \rho(k) S(k) \sqrt{T_{L R} T_{R D} T_{L S} T_{S D}} \sum_{j=1}^{J} \sum_{n=1}^{N}\left\{\sqrt{R_{R j} R_{S n}}\left[e^{i 2 k\left(z_{S_{n}}-z_{R j}\right)}+e^{-i 2 k\left(z_{S n}-z_{R j}\right)}\right]\right\}
\end{aligned}
$$

'Cross-correlation Terms'

$+\frac{1}{2} \rho(k) S(k) T_{L R} T_{R D} \sum_{\substack{j=1 \\ j \neq h}}^{J} \sum_{h=1}^{J}\left\{\sqrt{R_{R j} R_{R h}}\left[e^{i 2 k\left(z_{R j}-z_{R h}\right)}+e^{-i 2 k\left(z_{R j}-z_{R h}\right)}\right]\right\}$

'Auto-correlation Terms of Reference'

$+\frac{1}{2} \rho(k) S(k) T_{L S} T_{S D} \sum_{\substack{n=1 \\ n \neq m}}^{N} \sum_{m=1}^{N}\left\{\sqrt{R_{S n} R_{S m}}\left[e^{i 2 k\left(z_{S n}-z_{S m}\right)}+e^{-i 2 k\left(z_{S n}-z_{S m}\right)}\right]\right\}$

'Auto-correlation Terms of Sample',

where $R_{R j}=\left|r_{R j}\right|^{2}$ is the optical power reflectance of the discrete reference reflectors.

In the following section, we demonstrate how to retrieve the sample information from the cross-correlation terms and reduce or remove the influence from other terms.

The first half of the DC terms, and the auto-correlation terms of the reference are independent of the sample. Therefore, they can be considered as a background which can be determined by a measurement without the presence of a sample. This background can be subtracted in subsequent sample measurements, which results in a background-corrected current

$$
\begin{aligned}
I_{D c}(k)= & \rho(k) S(k) T_{L S} T_{S D}\left(R_{S 1}+R_{S 2}+\ldots\right) \\
& \text { 'DC Terms' } \\
+ & \rho(k) S(k) \sqrt{T_{L R} T_{R D} T_{L S} T_{S D}} \sum_{j=1}^{J} \sum_{n=1}^{N}\left\{\sqrt{R_{R j} R_{S n}}\left[e^{i 2 k\left(z_{s_{n}}-z_{R j}\right)}+e^{-i 2 k\left(z_{S_{n}}-z_{R j}\right)}\right]\right\}
\end{aligned}
$$

'Cross-correlation Terms'

$$
+\frac{1}{2} \rho(k) S(k) T_{L S} T_{S D} \sum_{\substack{n=1 \\ n \neq m}}^{N} \sum_{m=1}^{N}\left\{\sqrt{R_{S n} R_{S m}}\left[e^{i 2 k\left(z_{S n}-z_{S m}\right)}+e^{-i 2 k\left(z_{S n}-z_{S m}\right)}\right]\right\}
$$

'Auto-correlation Terms of Sample'.

The remaining DC terms can be separated after Fourier transformation of $I_{D c}(k)$ as shown in Fig. 2.2.2. The auto-correlation terms can be neglected in a typical biological tissue imaging application, since the reflectivity of the sample (on the order of $\sim 10^{-4}$ to $10^{-5}$ [1]) is much smaller than the reference. Thus the auto-correlation terms are small compared to the cross-correlation terms.

The cross-correlation terms can be rewritten as 


$$
I_{D c, \text { cross }}(k)=\rho(k) S(k) \sqrt{T_{L R} T_{R D} T_{L S} T_{S D}} \sum_{n=1}^{N}\left\{\sqrt{R_{R 1} R_{S n}}\left[e^{i 2 k\left(z_{S n}-z_{R 1}\right)}+e^{-i 2 k\left(z_{S n}-z_{R 1}\right)}\right]\right\}
$$

'From Reference reflection1'

$+\rho(k) S(k) \sqrt{T_{L R} T_{R D} T_{L S} T_{S D}} \sum_{n=1}^{N}\left\{\sqrt{R_{R 2} R_{S n}}\left[e^{i 2 k\left(z_{S n}-z_{R 2}\right)}+e^{-i 2 k\left(z_{S n}-z_{R 2}\right)}\right]\right\}$

'From Reference reflection 2'

$+\rho(k) S(k) \sqrt{T_{L R} T_{R D} T_{L S} T_{S D}} \sum_{n=1}^{N}\left\{\sqrt{R_{R 3} R_{S n}}\left[e^{i 2 k\left(z_{S n}-z_{R 3}\right)}+e^{-i 2 k\left(z_{S n}-z_{R 3}\right)}\right]\right\}$

'From Reference reflection 3' $+\ldots$.

The terms from reference reflection 1, first line in the Equation (2.3.4), can be expanded to:

$$
\begin{aligned}
I_{D c, \text { cross }, R 1}(k) & =\rho(k) S(k) \sqrt{T_{L R} T_{R D} T_{L S} T_{S D}}\left\{\sum_{n=1}^{N}\left[\sqrt{R_{R 1} R_{S n}} e^{i 2 k\left(z_{S n}-z_{R 1}\right)}\right]+\sum_{n=1}^{N}\left[\sqrt{R_{R 1} R_{S n}} e^{-i 2 k\left(z_{S n}-z_{R 1}\right)}\right]\right\} \\
& =\rho(k) S(k)\left[c_{S R 1}(k)+c_{S R 1}(k)^{*}\right]
\end{aligned}
$$

where * denotes the complex conjugate and

$$
c_{S R 1}(k)=\sqrt{T_{L R} T_{R D} T_{L S} T_{S D}} \sum_{n=1}^{N}\left\{\sqrt{R_{R 1} R_{S n}} e^{i 2 k\left(z_{S n}-z_{R 1}\right)}\right\} .
$$

The Fourier transform

$$
i_{D c, R 1}(z)=F\left[\rho(k) S(k) c_{S R 1}(k)+\rho(k) S(k) c_{S R 1}(k)^{*}\right]
$$

of $I_{D c, \text { cross }, R 1}(k)$, where $F[]$ denotes the Fourier transform, gives the sample signal of interest,

$$
i_{D c, R 1, \text { image }}(z)=F\left[\rho(k) S(k) c_{S R 1}(k)\right],
$$

as shown in Fig. 2.2.2 as the cross-correlation terms. However, this signal is mixed with all other cross-correlation terms generated by other reference reflectors. Additional processing is needed in order to separate the signal of interest from the mixture.

Assuming that all reference reflectors have a fixed reflectivity and location in the system, we can write

$$
z_{R 2}=z_{R 1}+\Delta z_{21}, \quad z_{R 3}=z_{R 1}+\Delta z_{31}, \ldots
$$

where $\Delta z_{j 1}$ is the optical distance of the $j^{\text {th }}$ reference reflector to the first reference reflector. Then the terms from reference reflection 2 can be expanded to [and by using Equation (2.3.6)]: 


$$
\begin{aligned}
& I_{D c, \text { cross }, 2}(k) \\
& =\rho(k) S(k) \sqrt{T_{L R} T_{R D} T_{L S} T_{S D}}\left\{\sum_{n=1}^{N}\left[\sqrt{R_{R 2} R_{S n}} e^{i 2 k\left(z_{S n}-z_{R 1}-\Delta z_{21}\right)}\right]+\sum_{n=1}^{N}\left[\sqrt{R_{R 2} R_{S n}} e^{-i 2 k\left(z_{S n}-z_{R 1}-\Delta z_{21}\right)}\right]\right\} \\
& =\rho(k) S(k) \sqrt{T_{L R} T_{R D} T_{L S} T_{S D}}\left\{\sum_{n=1}^{N}\left[\sqrt{R_{R 2} R_{S n}} e^{i 2 k\left(z_{S n}-z_{R 1}\right)}\right] e^{-i 2 k \Delta z_{21}}+\sum_{n=1}^{N}\left[\sqrt{R_{R 2} R_{S n}} e^{-i 2 k\left(z_{S n}-z_{R 1}\right)}\right] e^{i 2 k \Delta z_{21}}\right\} \\
& =\rho(k) S(k)\left[\frac{\sqrt{R_{R 2}}}{\sqrt{R_{R 1}}} c_{S R 1}(k) e^{-i 2 k \Delta z_{21}}+\frac{\sqrt{R_{R 2}}}{\sqrt{R_{R 1}}} c_{S R 1}(k)^{*} e^{i 2 k \Delta z_{21}}\right]
\end{aligned}
$$

The terms from the remaining reference reflections are treated similar to the terms from reference reflection 2. Then the cross-correlation terms in $I_{D c}(k)$ can be written as

$$
\begin{aligned}
& I_{D c, \text { cross }}(k) \\
& =\rho(k) S(k)\left[c_{S R 1}(k)+\sum_{j=2}^{J} \frac{\sqrt{R_{R j}}}{\sqrt{R_{R 1}}} c_{S R 1}(k) e^{-i 2 k \Delta z_{j 1}}+c_{S R 1}(k)^{*}+\sum_{j=2}^{J} \frac{\sqrt{R_{R j}}}{\sqrt{R_{R 1}}} c_{S R 1}(k)^{*} e^{\left.i 2 k \Delta z_{j 1}\right]}\right. \\
& =\rho(k) S(k)\left[c_{S R 1}(k)\left(1+\sum_{j=2}^{J} \frac{\sqrt{R_{R j}}}{\sqrt{R_{R 1}}} e^{-i 2 k \Delta z_{j 1}}\right)+c_{S R 1}(k)^{*}\left(1+\sum_{j=2}^{J} \frac{\sqrt{R_{R j}}}{\sqrt{R_{R 1}}} e^{i 2 k \Delta z_{j 1}}\right)\right] .
\end{aligned}
$$

The first half of the Equation (2.3.11), $\rho(k) S(k) c_{S R 1}(k)\left(1+\sum_{j=2}^{J} \frac{\sqrt{R_{R j}}}{\sqrt{R_{R 1}}} e^{-i 2 k \Delta z_{j 1}}\right)$, means that each sample reflection peak in the Fourier transformed detector current spectrum for the ideal single reference reflector case, $i_{D c, R 1, \text { image }}(z)$ will for the multi-reference reflector case result in $J$ peaks with a fixed relative amplitude and location shift, regardless of the absolute location of the real signal peak. This leads to a blur of the OCT image. The second half of the Equation (2.3.11), $\rho(k) S(k) c_{S R 1}(k)^{*}\left(1+\sum_{j=2}^{J} \frac{\sqrt{R_{R j}}}{\sqrt{R_{R 1}}} e^{i 2 k \Delta z_{j 1}}\right)$, describes the mirrored artifacts of the first half, which has opposite peak-location shift with respect to the first half.

If we set

$$
\alpha(k)=\left(1+\sum_{j=2}^{J} \frac{\sqrt{R_{R j}}}{\sqrt{R_{R 1}}} e^{-i 2 k \Delta z_{j 1}}\right)
$$

then the cross-correlation terms of $I_{D c}(k)$ can be written as:

$$
I_{D c, \text { cross }}(k)=\rho(k) S(k)\left[c_{S R 1}(k) \alpha(k)+c_{S R 1}(k)^{*} \alpha(k)^{*}\right]
$$

and its Fourier transform is 
$i_{D c}(z)=F\left[\rho(k) S(k) c_{S R 1}(k)\right] \otimes F[\alpha(k)]+F\left[\rho(k) S(k) \mathrm{c}_{S R 1}(k)^{*}\right] \otimes F\left[\alpha(k)^{*}\right]$

where $\otimes$ denotes convolution. This means the blurred image,

$$
i_{D c, \text { image }}(z)=F\left[\rho(k) S(k) c_{S R 1}(k)\right] \otimes F[\alpha(k)]=i_{D c, R 1, \text { image }}(z) \otimes F[\alpha(k)],
$$

can be deblurred to $i_{D c, R 1, \text { image }}(z)$ with a deconvolution technique as long as the mirrored image (artifacts),

$$
i_{D c, \text { artifacts }}(z)=F\left[\mathrm{c}_{S R 1}(k)^{*} \rho(k) S(k)\right] \otimes F\left[\alpha(k)^{*}\right],
$$

is well separated from the real image.

The sample signal of interest, $i_{D c, R 1, \text { image }}(z)$, from Equation (2.3.8) can be written as

$$
i_{D c, R 1, \text { image }}(z)=F\left[\rho(k) S(k) c_{S R 1}(k)\right]=F[\rho(k) S(k)] \otimes F\left[c_{S R 1}(k)\right],
$$

which can be understood as a convolution of the Dirac comb function, $F\left[c_{S R 1}(k)\right]$, from the discrete sample reflector, and a broadening function, $F[\rho(k) S(k)]$, due to the limited spectral width of the light source and the detector. The broadening function $F[\rho(k) S(k)]$ can be measured independently for the OCT system, since it is only related to the light source and the detector. The transfer function $F[\alpha(k)]$ of the multi-reference system in Equation (2.3.15) can be measured with a single reflection sample (such as a mirror) at a given location, where its mirrored artifacts, $i_{D c \text {,artifacts }}(z)$, are well separated from its signal $i_{D c, \text { image }}(z)$. Equation (2.3.15) can be written as

$$
i_{D c, \text { image }}(z)=F[\rho(k) S(k)] \otimes F\left[c_{S R 1}(k)\right] \otimes F[\alpha(k)]
$$

where $F\left[c_{S R 1}(k)\right]$ is a delta function in this case. We then can determine the transfer function $F[\alpha(k)]$ in our system with the knowledge of the broadening function $F[\rho(k) S(k)]$. This transfer function $F[\alpha(k)]$ then can be used to deconvolve an image of any real sample.

An alternative way of deconvolving an image is to consider

$$
H_{P S F}=F[\rho(k) S(k)] \otimes F[\alpha(k)]
$$

as the point spread function (PSF) of the system. This PSF can be obtained directly from a mirror sample measurement. Deconvolution with this PSF could even sharpen the image to a certain degree. This alternative deconvolution approach is used in the Section 5.6 (experimental data processing). 


\subsection{Noise in an FD-OCT system}

In this section, we distinguish two categories of noise in an FD-OCT system, namely the noise from the measurement and the noise introduced during the signal processing. The total noise in a measurement mainly consists of detector read-out noise, shot noise and relative intensity noise (RIN). All those noise contributions have been extensively studied [1-7] and can be summarized as follows:

$$
\sigma_{\text {measured }}^{2}=\sigma_{\text {read }}^{2}+\sigma_{\text {shot }}^{2}+\sigma_{R I N}^{2},
$$

where the additive variance terms $\sigma^{2}$ are proportional to the energy of the respective noise components in the detected signal during a given integration time, and are expressed in units of charge squared $\left[\mathrm{C}^{2}\right.$ or $\left.(\mathrm{A} \cdot \mathrm{s})^{2}\right]$.

The read-out noise $\sigma_{\text {read }}^{2}$ of a detector includes the dark-current noise, thermal noise and quantization noise [4] which do not depend on the signal in general.

The shot noise $\sigma_{\text {shot }}^{2}$ arises from the statistical nature of photons. If $N_{p}$ is the average number of photons incident on a detector within an integration time $\tau_{i}$, the standard deviation in $N_{p}$ is equal to $\sqrt{N_{p}} . N_{p}$ can be calculated as

$$
N_{p}=\frac{P \tau_{i}}{E_{v}}
$$

where $P$ is the average optical power on the detector. The photon energy $E_{v}$ can be calculated as

$$
E_{v}=h v=\frac{h c}{\lambda}=\frac{h c k}{2 \pi}
$$

where $h$ is the Planck constant, $v$ is the frequency of the photon and $\lambda$ is the wavelength of the photon. The number of electrons generated in this detector is

$$
N_{e}=\eta(k) N_{p}
$$

where $\eta(k)$ is the quantum efficiency of the detector, which is a function of the wavenumber. The quantum efficiency can be linked to the responsivity $\rho(k)$ in Section 2.2 by

$$
\rho(k)=\frac{I}{P}=\frac{N_{e} e}{N_{p} E_{v}}=\eta(k) \frac{e}{E_{v}},
$$

where $e$ is the charge of an electron. The fluctuation in the number of electrons is $\sqrt{N_{e}}$, thus 
the standard deviation in the detected charge is calculated as:

$$
\sigma_{\text {shot }}=\sqrt{N_{e}} e .
$$

Using Equations (2.4.6), Equation (2.4.5) and Equation (2.4.2), the shot noise on the detector within an integration time of $\tau_{i}$ can then be calculated as

$$
\sigma_{\text {shot }}^{2}=N_{e} e^{2}=\rho(k) P \tau_{i} e .
$$

The relative intensity noise (RIN), $\sigma_{R I N}^{2}$, arises from the instability of the light source. Many studies $[1,3,4]$ have shown that the RIN can be calculated from the coherence time $\tau_{c o h}$ of the light incident on the detector as

$$
\sigma_{R I N}^{2}=\left[\frac{\eta(k) e P}{E_{v}}\right]^{2} \tau_{i} \tau_{c o h}=[\rho(k) P]^{2} \tau_{i} \tau_{c o h}
$$

However, Shin et al. [6] have indicated that this formula should only be used in case of an purely spontaneous emission light source. For other cases, such as a sweeping laser in an SSOCT system, an empirical noise suppression factor $\eta_{n s}$ (which is dependent on the individual light source) should be introduced into Equation (2.4.8), resulting in

$$
\sigma_{R I N}^{2}=[\rho(k) P]^{2} \tau_{i} \frac{\tau_{c o h}}{\eta_{n s}}
$$

In practice, the RIN of a particular light source may be obtained from its specifications, or it should be measured (e.g. using the method described in [6]).

The noise introduced during the signal processing is a result of the side lobes in the Fourier transform of the measured spectrum $I_{D}(k)$. The magnitude of this noise is largely dependent on the shape of the source spectrum. For instance, a light source with a rectangular spectrum produces larger side lobes, thus more noise, than a source with an Gaussian spectrum. By applying a windowing function on the measured spectrum $I_{D}(k)$, one can reduce the magnitude of this side-lobe noise. However, the use of a windowing function affects not only the side-lobe noise but also the amplitude of the signal and the axial resolution. Therefore, the selection of the windowing function is a tradeoff between the SNR and the axial resolution. In Chapter 5, we introduce an alternative way, local mean removal, to suppress the side-lobe noise without sacrificing the axial resolution. 


\subsection{Signal-to-noise ratio (SNR) in an FD-OCT system}

In this section, we first clarify the definition of the SNR in an A-scan given by $i_{D}(z)$, that we will use throughout this thesis. We then discuss the effect of the Fourier transform on the signal and the noise, finally arriving at a formula for the shot-noise limited SNR.

In this thesis, we only quantify the SNR in the case of a single-reflector sample, such as a mirror. For that case we define the SNR of the A-scan as

$$
S N R=\frac{i_{D}^{2}\left(z_{p}\right) \tau_{i}^{2}}{\sigma^{2}},
$$

where $\left|i_{D}\left(z_{p}\right) \tau_{i}\right|$ is the peak height of the A-scan at the sample location $z_{p}$ (in units of Coulomb), $\tau_{i}$ is the integration time and $\sigma$ is the standard deviation in $\left|i_{D}(z) \tau_{i}\right|$ at positions far away from the peak position $z_{p}$. The value of the SNR is often expressed in $\mathrm{dB}$ units, which can be calculated as

$$
S N R_{d B}=10 \log _{10}\left(\frac{i_{D}^{2}\left(z_{p}\right) \tau_{i}^{2}}{\sigma^{2}}\right)=20 \log _{10}\left(\frac{\left|i_{D}\left(z_{p}\right) \tau_{i}\right|}{\sigma}\right) .
$$

Examples of using this definition for the SNR are found in Chapter 5.

To simplify the analysis of the SNR, we consider a single reference plane as described in Section 2.2. The source power spectral density $S(k)$ in Equation (2.2.7) can be integrated over each spectral channel $k_{m}$, giving the power $P\left(k_{m}\right)$ for each channel. Thus the current $I_{D}\left(k_{m}\right)$ measured in each spectral channel becomes (no auto-correlation terms for singlereflector sample)

$$
\begin{aligned}
I_{D}\left(k_{m}\right)= & \rho\left(k_{m}\right)\left[P_{R}\left(k_{m}\right)+P_{S}\left(k_{m}\right)\right] \\
& \text { DC Terms' } \\
+ & \rho\left(k_{m}\right) \sqrt{P_{R}\left(k_{m}\right) P_{S}\left(k_{m}\right)}\left[e^{i 2 k_{m}\left(z_{R}-z_{S}\right)}+e^{-i 2 k_{m}\left(z_{R}-z_{S}\right)}\right]
\end{aligned}
$$

'Cross-correlation Terms'

where

$$
P_{R}\left(k_{m}\right)=P\left(k_{m}\right) T_{L R} T_{R D} R_{R} \text { and } P_{S}\left(k_{m}\right)=P\left(k_{m}\right) T_{L S} T_{S D} R_{S}
$$

are the powers at the detector for spectral channel $m$ due to reflection from respectively the reference and the sample. The spectral channel width is defined as the spectral width incident on a single pixel of the spectrometer in the case of a spectral domain OCT, or the laser swept 
range during the integration time $\tau_{i}$ in the case of a swept source OCT. Note that the units of $I_{D}\left(k_{m}\right)$ [A] are different from those of $I_{D}(k)[\mathrm{A} / \mathrm{Hz}]$ in Sections 2.2 and 2.3. The detected charges accumulate during the integration time $\tau_{i}$ to

$$
Q\left(k_{m}\right)=I_{D}\left(k_{m}\right) \tau_{i}
$$

A discrete-time Fourier transform is used to calculate the A-scan charge

$$
q_{D}(z)=\sum_{m=1}^{M} I_{D}\left(k_{m}\right) \tau_{i} e^{-i 2 k_{m} z}=i_{D}(z) \tau_{i}
$$

where $M$ is the total number of spectral channels. The value of $i_{D}(z)$ is calculated with Equations (2.5.3) and (2.5.6) as

$$
\begin{aligned}
& i_{D}(z)=\sum_{m=1}^{M} I_{D}\left(k_{m}\right) e^{-i 2 k_{m} z} \\
& =\sum_{m=1}^{M} \rho\left(k_{m}\right)\left[P_{R}\left(k_{m}\right)+P_{S}\left(k_{m}\right)\right] e^{-i 2 k_{m} z} \quad \text { 'DC Terms' } \\
& +\sum_{m=1}^{M} \rho\left(k_{m}\right) \sqrt{P_{R}\left(k_{m}\right) P_{S}\left(k_{m}\right)} e^{i 2 k_{m}\left(z_{R}-z_{S}-z\right)} \quad \text { 'Mirror image artifacts' } \\
& +\sum_{m=1}^{M} \rho\left(k_{m}\right) \sqrt{P_{R}\left(k_{m}\right) P_{S}\left(k_{m}\right)} e^{-i 2 k_{m}\left(z_{R}-z_{S}+z\right)} \quad \text { 'Cross-correlation signal'. }
\end{aligned}
$$

At the signal peak location $z=z_{p}=z_{S}-z_{R}$. With sufficiently large $M$ (in practice typically a few hundred or more), the summation of the DC terms and the mirror image artifacts in Equation (2.5.7) are negligibly small (summation over non zero phase) compared to the summation of the cross-correlation signal terms (summation over real numbers). Therefore, the value of the cross-correlation signal peak $i_{D}\left(z_{p}\right)$ is

$$
i_{D}\left(z_{p}=z_{S}-z_{R}\right)=\sum_{m=1}^{M} \rho\left(k_{m}\right) \sqrt{P_{R}\left(k_{m}\right) P_{S}\left(k_{m}\right)}=M \rho \sqrt{P_{R} P_{S}},
$$

where the latter expression assumes that all spectral channels have equal power and equal responsivity on the detector. Thus, the peak height squared of the signal is

$$
i_{D}^{2}\left(z_{p}\right) \tau_{i}^{2}=M^{2} \rho^{2} P_{R} P_{S} \tau_{i}^{2}
$$

This shows that the signal adds up coherently (all the cross-correlation signal terms in Equation (2.5.7) have the same zero phase) under the Fourier transform.

On the other hand, the noise is adding up incoherently (all the noise terms have random phases with respect to each other) under the Fourier transform. In case of a shot-noise limited 
system, the noise after the Fourier transform can be calculated from Equation (2.4.7), resulting in [1]

$$
\sigma^{2}=M \rho P \tau_{i} e
$$

where $P$ is the total optical power in one spectral channel $P\left(k_{m}\right)$, assuming equal power and equal detector responsivity in all spectral channels. This assumption can only be used in the case that the cross-correlation terms in Equation (2.5.3) are negligible compared to the DC terms, which means $P_{R} \gg P_{S}$ or $P_{R}<<P_{S}$. By neglecting the cross-correlation terms in Equation (2.5.3), the total optical power can be calculated as

$$
P=P_{R}+P_{S}
$$

The SNR for a shot noise limited system can be calculated by inserting Equations (2.5.9), (2.5.10), and (2.5.11) into Equation (2.5.1), resulting in

$$
S N R=\frac{\rho M \tau_{i}}{e} \frac{P_{R} P_{S}}{P_{R}+P_{S}} .
$$

A simpler formula has been shown in several studies [2,3] which is only suitable for the case of $P_{R} \gg P_{S}$. We have kept the derivation more general. Thus, this formula can be used in both cases of $P_{R} \gg P_{S}$ and $P_{R}<<P_{S}$.

In the case of a non-shot-noise-limited system, the SNR depends on the technical details of the experimental setup, which includes the RIN of the light source, the read-out noise of the detector and the side-lobe noise from the Fourier transform. The influence of these different noise sources is discussed in the experimental section in Chapter 5.

\subsection{Influence on SNR by optical losses}

The performance of OCT is typically hampered by the very weak scattering signal from tissue, thus the optical losses of all the components should be as small as possible to keep the optical power efficiency of the system as high as possible. The final OCT image quality depends on SNR, thus, a quantitative analyze of the SNR influenced by the optical losses is presented in this section.

The square of the OCT signal can be calculated using Equation (2.5.9) as

$$
i_{D}^{2}\left(z_{p}\right) \tau_{i}^{2}=M^{2} \rho^{2} P_{R} P_{S} \tau_{i}^{2}
$$

In an OCT measurement we can identify three different noise sources as discussed in Section 2.4. Depending on which noise source is the dominating one, the optical loss has a 
different influence on the SNR. All three cases are discussed below.

In the first case is the noise is dominated by the detector read-out noise. The detector read-out noise is independent of the optical power in the system. Therefore, the SNR is proportional to the square of the OCT signal and thus proportional to $P_{R} P_{S}$. Using Equation (2.5.4) we find:

$$
S N R \propto P_{R} P_{S} \propto T_{L R} T_{R D} T_{L S} T_{S D}
$$

where $T_{L R}, T_{R D}, T_{L S}$ and $T_{S D}$ are the optical power transmittance from light source to reference reflector, from reference reflector to detector, from light source to sample and from sample to detector, respectively. Considering our common-path system design in Fig.1.4.1 as an example, there are some optical losses $\left(\zeta_{R S n}\right)$ that influence both $P_{R}$ and $P_{S}$ (through $T_{L R}$ and $T_{R D}$ ) and others ( $\zeta_{S m}$ ) only influence $P_{S}$ (through $T_{R S}$ and $T_{S R}$ ), where $T_{R S}$ and $T_{S R}$ are the optical power transmittance from the reference reflector to the sample and from the sample to the reference reflector, respectively. Thus

$$
\begin{aligned}
& P_{R} \propto T_{L R} T_{R D}=\left(1-\zeta_{R S 1}\right)\left(1-\zeta_{R S 2}\right) \cdots\left(1-\zeta_{R S n}\right), \\
& P_{S} \propto T_{L R} T_{R D} \cdot T_{R S} T_{S R}=\left(1-\zeta_{R S 1}\right)\left(1-\zeta_{R S 2}\right) \cdots\left(1-\zeta_{R S n}\right) \cdot\left(1-\zeta_{S 1}\right)\left(1-\zeta_{S 2}\right) \cdots\left(1-\zeta_{S m}\right),
\end{aligned}
$$

where 1 to $n$ and 1 to $m$ represent different optical elements. Therefore, any optical loss $\left(\zeta_{R S n}\right)$ introduced between the light source, end facet of the waveguide (reference reflector), and the detector reduces the SNR (in the $\mathrm{dB}$ scale) by

$$
\Delta S N R_{R S n_{-} d B}=20 \log _{10}\left(1-\zeta_{R S n}\right) .
$$

An optical loss $\left(\zeta_{S m}\right)$ between the reference reflector and the sample reduces the SNR (in the dB scale) by

$$
\Delta S N R_{S m_{-} d B}=10 \log _{10}\left(1-\zeta_{S m}\right)
$$

The second case is the total noise dominated by the RIN. The RIN, $\sigma_{R I N}^{2}$, is proportional to the square of the total optical power at the detector, see Equation (2.4.9). Together with Equation (2.5.11) and Equation (2.6.1) we find for the SNR:

$$
S N R \propto \frac{P_{R} P_{S}}{\left(P_{R}+P_{S}\right)^{2}} .
$$

In a typical biological application, $P_{R} \gg P_{S}$, thus,

$$
S N R \propto \frac{P_{S}}{P_{R}} \propto\left(1-\zeta_{S 1}\right)\left(1-\zeta_{S 2}\right) \cdots\left(1-\zeta_{S m}\right)
$$

This means only the optical loss of $\zeta_{S m}$ in between the reference reflector and the sample 
reduces the $\mathrm{SNR}$ (in the $\mathrm{dB}$ scale) by

$$
\Delta S N R_{S m_{-} d B}=10 \log _{10}\left(1-\zeta_{S m}\right) .
$$

The optical loss of $\zeta_{R S n}$ does not affect the SNR.

In the case of a strongly reflecting sample, where $P_{R}<<P_{S}$,

$$
S N R \propto \frac{P_{R}}{P_{S}} \propto \frac{1}{\left(1-\zeta_{S 1}\right)\left(1-\zeta_{S 2}\right) \cdots\left(1-\zeta_{S m}\right)} .
$$

This means the optical loss of $\zeta_{S m}$ counterintuitively improves the SNR (in the dB scale) by

$$
\Delta S N R_{S m_{-} d B}=-10 \log _{10}\left(1-\zeta_{S m}\right) .
$$

This is due to the fact that the magnitude of the noise reduces more than that of the signal. The optical loss of $\zeta_{R S n}$ still does not have any influence to the SNR.

The third case describes the situation in which the total noise is dominated by the shot noise. The SNR in a shot noise limited case is calculated using Equation (2.5.12) as:

$$
S N R \propto \frac{P_{R} P_{S}}{P_{R}+P_{S}} .
$$

In a typical biological application, $P_{R} \gg P_{S}$, thus,

$$
S N R \propto P_{S} \propto\left(1-\zeta_{R S 1}\right)\left(1-\zeta_{R S 2}\right) \cdots\left(1-\zeta_{R S n}\right) \cdot\left(1-\zeta_{S 1}\right)\left(1-\zeta_{S 2}\right) \cdots\left(1-\zeta_{S m}\right) .
$$

This means all optical losses of $\zeta_{R S n}$ and $\zeta_{S m}\left(\zeta_{j}\right)$ result in the same reduction in SNR by

$$
\Delta S N R_{\mathrm{j}_{\_} d B}=10 \log _{10}\left(1-\zeta_{j}\right) .
$$

In case of a strong reflecting sample, where $P_{R}<<P_{S}$,

$$
S N R \propto P_{R} \propto\left(1-\zeta_{R S 1}\right)\left(1-\zeta_{R S 2}\right) \cdots\left(1-\zeta_{R S n}\right) .
$$

This means the optical loss of $\zeta_{R S n}$ reduces the SNR by

$$
\Delta S N R_{R S n_{-} d B}=10 \log _{10}\left(1-\zeta_{R S n}\right) .
$$

The optical loss of $\zeta_{S m}$ does not have any influence on the SNR.

The results discussed above are summarized in Table 2.6.1. 
Table 2.6.1. Overview of the expected influence of optical losses on the SNR for different noise conditions.

\begin{tabular}{|c|c|c|c|}
\hline $\begin{array}{l}\text { Dominating } \\
\text { noise }\end{array}$ & $\begin{array}{c}\text { Reference and sample } \\
\text { power relation }\end{array}$ & $\begin{array}{c}\text { Component loss } \\
(\%)\end{array}$ & $\Delta S N R_{d B}$ \\
\hline \multirow{2}{*}{$\begin{array}{l}\text { Read-out } \\
\text { noise }\end{array}$} & \multirow{2}{*}{1} & $\zeta_{R S n}$ & $20 \log _{10}\left(1-\zeta_{R S n}\right)$ \\
\hline & & $\zeta_{S m}$ & $10 \log _{10}\left(1-\zeta_{S m}\right)$ \\
\hline \multirow{4}{*}{$\begin{array}{c}\text { Relative } \\
\text { intensity } \\
\text { noise }\end{array}$} & \multirow{2}{*}{$P_{R} \gg P_{S}$} & $\zeta_{R S n}$ & No influence \\
\hline & & $\zeta_{S m}$ & $10 \log _{10}\left(1-\zeta_{S m}\right)$ \\
\hline & \multirow{2}{*}{$P_{R}<<P_{S}$} & $\zeta_{R S n}$ & No influence \\
\hline & & $\zeta_{S m}$ & $-10 \log _{10}\left(1-\zeta_{S m}\right)$ \\
\hline \multirow{4}{*}{ Shot noise } & \multirow{2}{*}{$P_{R} \gg P_{S}$} & $\zeta_{R S n}$ & $10 \log _{10}\left(1-\zeta_{R S n}\right)$ \\
\hline & & $\zeta_{S m}$ & $10 \log _{10}\left(1-\zeta_{S m}\right)$ \\
\hline & \multirow{2}{*}{$P_{R}<<P_{S}$} & $\zeta_{R S n}$ & $10 \log _{10}\left(1-\zeta_{R S n}\right)$ \\
\hline & & $\zeta_{S m}$ & No influence \\
\hline \multicolumn{4}{|c|}{$\begin{array}{l}P_{R}: \text { Reflected reference power on the detector without the present of any sample. } \\
P_{S}: \text { Reflected sample power on the detector without the present of reference reflector. } \\
\zeta_{R S n}: \text { Optical losses in between the light source, end facet of the waveguide (reference reflector) and the } \\
\text { detector. } \\
\zeta_{S m}: \text { Optical losses in between reference reflector and the sample. }\end{array}$} \\
\hline
\end{tabular}

The Y junction influences the SNR not only through its losses but also its power splitting ratio. Thus, we provide a discussion of the influence to the SNR by both these properties of the $\mathrm{Y}$ junction. To simplify the discussion, we again take the system in Fig.1.4.1 as an example and only consider the shot noise limited case. The following discussion can be easily adjusted in case of replacing the $\mathrm{Y}$ junction by a directional coupler, an MMI or a circulator.

Assuming the light comes from port 1 in Fig. 1.4.1, the optical loss of the Y junction is defined as:

$$
\zeta_{Y}=\left(P_{\text {port } 1}-P_{\text {port } 2}-P_{\text {port } 3}\right) / P_{\text {port } 1} \times 100 \%
$$

and the power ratio of port 2 is defined as

$$
\gamma_{2}=\frac{P_{p o r t 2}}{P_{\text {port } 2}+P_{p o r t 3}} .
$$

The power ratio of port 3 is $\gamma_{3}=1-\gamma_{2}$. Since the $\mathrm{Y}$ junction is a reciprocal optical 
component and the light passes through the $\mathrm{Y}$ junction twice, the optical power transmittance of the Y junction (for the OCT system) can be calculated with

$$
T_{Y}=\gamma_{2} \cdot \gamma_{3} \cdot\left(1-\zeta_{Y}\right)^{2}
$$

Figure 2.6.1 shows the dependence of the power transmittance $T_{Y}$ on the power ratio $\gamma_{2}$ for different values of the optical loss. The maximum efficiency is 0.25 when the loss of the $Y$ junction is 0 and with an ideal 50/50 splitting ratio. The effective loss of the $\mathrm{Y}$ junction is

$$
\zeta_{\text {eff_Y }}=1-T_{Y}
$$

which influences both $P_{R}$ and $P_{S}$, thus is one of $\zeta_{R S n}$. Based on Table 2.6.1, the corresponding $\triangle S N R$ in case of a shot noise limited system are also plotted in Figure 2.6.1. It shows that a $8 \%$ optical loss (a typical loss expected for a Y-junction at wavelength of $1.3 \mu \mathrm{m}$, see Fig. 3.4.3) in the Y junction with $0.5 \pm 0.1$ power ratio reduces the SNR by less than $1 \mathrm{~dB}$ compared with the optimal case $(-6 \mathrm{~dB})$. Considering the typical SNR values of $100 \mathrm{~dB}$ in a high-quality FD-OCT [1], a $1 \mathrm{~dB}$ reduction is acceptable.

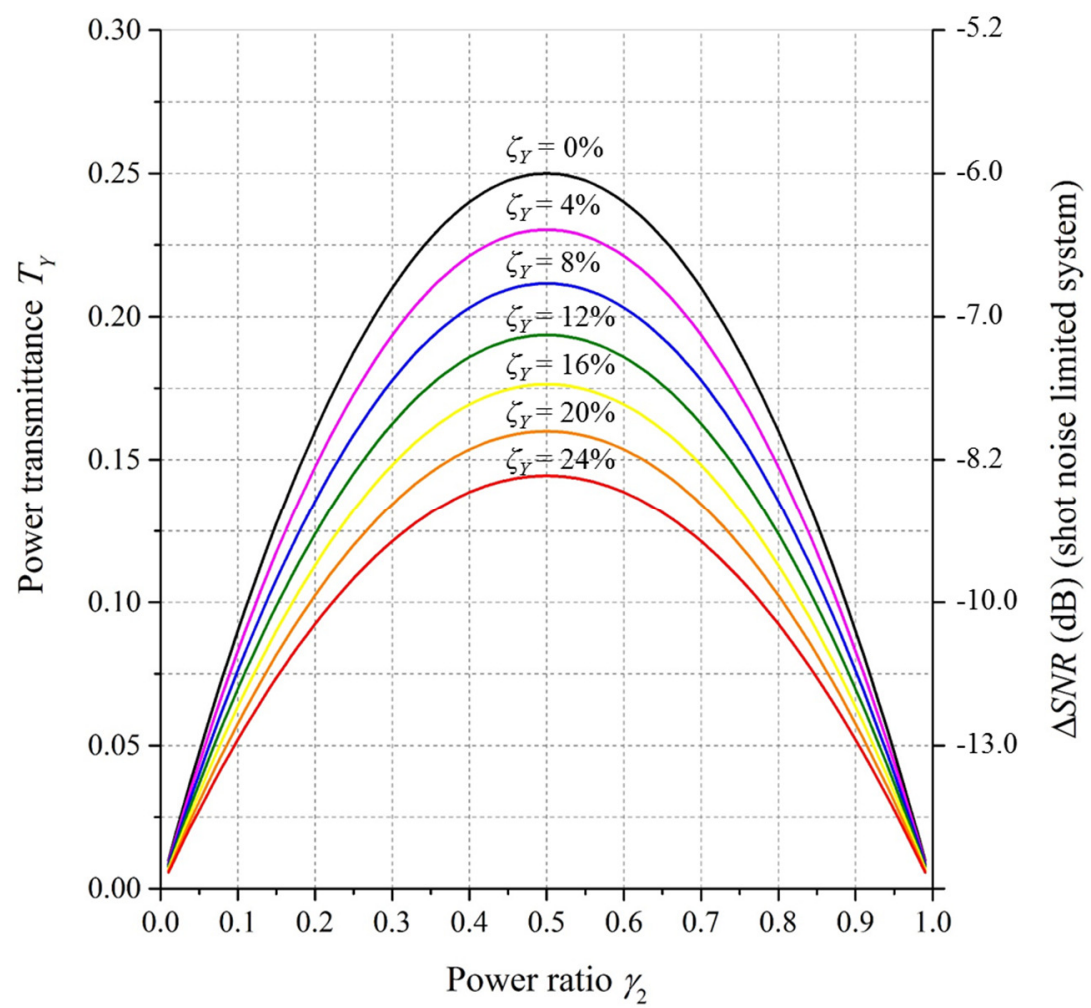

Fig. 2.6.1. Calculated $Y$ junction optical power transmittances for the OCT system $T_{Y}$ as a function of the loss $\zeta_{Y}$ and power ratio $\gamma_{2}$. The right axis indicate the $\Delta S N R$ by the Y junction in a shot noise limited system. 
To summarize, this section provides a quantitative discussion of the $\Delta \mathrm{SNR}$ as a function of optical loss under different noise conditions. The $\mathrm{Y}$ junction is a special component which influences the $\triangle$ SNR not only by the optical loss but also by its power splitting ratio. Thus, a detailed analysis of the $\mathrm{Y}$ junction is presented as well. The results presented here are useful when optimizing the design of the overall OCT system.

\subsection{Summary}

In this chapter, we have presented a mathematical model of the FD-OCT and extended the model to a system with multiple reference reflectors. We then presented a discussion of the different kinds of noise in an OCT system. The SNR of a shot noise limited system has been discussed in detail. Finally, the influences on the SNR by optical losses are discussed. This knowledge is not only useful during the designing of the system, but also important during the data analysis. 


\section{References}

1. Drexler, W. and J.G. Fujimoto, Optical Coherence Tomography: Technology and Applications. 2008: Springer.

2. Choma, M.A., M.V. Sarunic, C.H. Yang, and J.A. Izatt, Sensitivity advantage of swept source and Fourier domain optical coherence tomography. Optics Express, 2003. 11(18): p. 2183-2189.

3. de Boer, J.F., B. Cense, B.H. Park, M.C. Pierce, G.J. Tearney, and B.E. Bouma, Improved signal-tonoise ratio in spectral-domain compared with time-domain optical coherence tomography. Optics Letters, 2003. 28(21): p. 2067-2069.

4. Goldberg, B.D., B.J. Vakoc, W.Y. Oh, M.J. Suter, S. Waxman, M.I. Freilich, B.E. Bouma, and G.J. Tearney, Performance of reduced bit-depth acquisition for optical frequency domain imaging. Optics Express, 2009. 17(19): p. 16957-16968.

5. Leitgeb, R., C.K. Hitzenberger, and A.F. Fercher, Performance of fourier domain vs. time domain optical coherence tomography. Optics Express, 2003. 11(8): p. 889-894.

6. Shin, S., U. Sharma, H.H. Tu, W. Jung, and S.A. Boppart, Characterization and Analysis of Relative Intensity Noise in Broadband Optical Sources for Optical Coherence Tomography. Ieee Photonics Technology Letters, 2010. 22(14): p. 1057-1059.

7. Sorin, W.V. and D.M. Baney, A Simple Intensity Noise-Reduction Technique for Optical LowCoherence Reflectometry. Ieee Photonics Technology Letters, 1992. 4(12): p. 1404-1406. 


\section{Chapter 3}

\section{Design, fabrication and characterization of waveguide structures}

\subsection{Introduction}

This chapter introduces the waveguide elements used in the chip-based common-path OCT system. We first introduce the wavelength range of interest, which has to be considered during the design of these components. In Section 3.2, we briefly discuss the silicon oxynitride ( $\mathrm{SiON}$ ) waveguide technology, mentioning the typical waveguide dimensions and the key fabrication steps. In Section 3.3, we describe the design of an adiabatic bend structure, which ensures a low bending loss. In Section 3.4, we discuss the design and characterization of a modified $\mathrm{Y}$ junction, which has a reduced sensitivity to fabrication errors and is optimized to minimize losses.

The choice of the working wavelength range is based on the application and the available light sources. As mentioned in Section 1.1, we are mainly interested in skin imaging, thus infrared light is preferable due to its larger penetration depth compared to visible light. It is common to use a swept source centered at a wavelength of $\sim 1300 \mathrm{~nm}$ for skin imaging. Recently also a swept source centered at $\sim 1550 \mathrm{~nm}$ was developed for OCT applications [1]. The typical wavelength range of these two types of sources are $1260 \mathrm{~nm}-1360 \mathrm{~nm}$ [2] and $1485 \mathrm{~nm}-1600 \mathrm{~nm}$ [1]. It is interesting to design the chip such that it can be used at one of these wavelength bands. In addition, it would be favorable if the chip can work with a broadband source (such as SuperK OCT broadband sources from NKTphotonics) covering the wavelength range from $1260 \mathrm{~nm}$ to $1600 \mathrm{~nm}$ if high axial resolution OCT is required in future applications. Therefore, we define our working wavelength range to be $1200 \mathrm{~nm}-$ $1640 \mathrm{~nm}$ (with some margin compared with the light source range). 


\subsection{Silicon oxynitride waveguide technology}

All waveguide devices discussed throughout this thesis were realized with SiON waveguide technology [3]. There are many waveguide technologies with different materials suitable for OCT applications, such as silicon-on-insulator (SOI) [4], $\mathrm{Si}_{3} \mathrm{~N}_{4}$ [5], $\mathrm{SiON}$ [6] and ion exchange in glass [7]. The motivation for choosing SiON-based waveguides are not only the excellent optical properties, which fulfills the OCT requirements mentioned in Section 1.2.2, but also the availability of reliable fabrication tools in the cleanroom at the MESA+ Institute for Nanotechnology.

$\mathrm{SiON}$ is highly transparent over a large wavelength range from $0.2 \mu \mathrm{m}$ to $2.5 \mu \mathrm{m}$ which completely covers our relevant wavelength range $(1200 \mathrm{~nm}-1640 \mathrm{~nm})$. Optical losses as low as $0.08 \mathrm{~dB} / \mathrm{cm}$ at a wavelength of $1560 \mathrm{~nm}$ have been reported for SiON planar and channel waveguides [8]. The refractive index of $\mathrm{SiON}$ is tunable between 1.46 and 2.0 by changing the ratio of the oxygen and nitrogen in the material [9]. This tunable refractive index leads to a large flexibility in designing the properties of the waveguide.

The requirement from our common-path OCT system with respect to the waveguide design is that the waveguide should only support the fundamental transverse electric (TE) mode and fundamental transverse magnetic (TM) mode in the relevant wavelength range. This is important to keep the 50/50 splitting ratio of the $\mathrm{Y}$ junction (see Section 3.4). Many waveguide geometries fulfill this requirement. We chose a geometry which has been used in [10] and that fulfills the requirements mentioned above. The waveguide geometry is shown in Fig. 3.2.1. The structure is fabricated on a Si wafer (substrate) with a thickness of $500 \mu \mathrm{m}$. An $8 \mu \mathrm{m}$ thick $\mathrm{SiO}_{2}$ layer on top of the $\mathrm{Si}$ substrate serves as the bottom cladding to prevent leakage of light into the Si substrate. The SiON core has a width of $2 \mu \mathrm{m}$ and a height of $0.6 \mu \mathrm{m}$. The top cladding is a $4 \mu \mathrm{m}$ thick $\mathrm{SiO}_{2}$ layer. The refractive index of the $\mathrm{SiON}$ (core) and the $\mathrm{SiO}_{2}$ cladding (both bottom and top) are 1.55 and 1.45 respectively at a wavelength of $1.3 \mu \mathrm{m}$. The corresponding refractive index dispersion property is discussed in detail in [11]. We use the dispersion data in [11] as the input for the material refractive index in all the calculations and simulations discussed in this thesis.

The fabrication of the $\mathrm{SiON}$ waveguides is based on the $\mathrm{SiON}$ technology developed in the Integrated Optical MicroSystems group at the University of Twente [12]. The starting point is a $500 \mu \mathrm{m}$ thick $<100>$ silicon wafer with the diameter of $100 \mathrm{~mm}$. This wafer is wet thermal oxidized to form an $8 \mu \mathrm{m}$ thick $\mathrm{SiO}_{2}$ bottom cladding. On top of this layer, a SiON layer is deposited by plasma-enhanced chemical vapor deposition (PECVD), followed by a thermal annealing step for 3 hours at $1100^{\circ} \mathrm{C}$. The channel waveguides are fabricated by standard lithography in combination with reactive ion etching (RIE) utilizing a $\mathrm{CHF}_{3} / \mathrm{O}_{2}$ chemistry. A scanning electron microscope (SEM) image of an etched waveguide (before the growth of the top cladding) is shown in Fig. 3.2.2, which is in good agreement with our 
designed shape. Finally, the top cladding is deposited. The top cladding is composed of a $1 \mu \mathrm{m}$ thick $\mathrm{SiO}_{2}$ layer grown by low-pressure chemical vapor deposition (LPCVD) using tetraethyl orthosilicate (TEOS) and a $3 \mu \mathrm{m}$ thick PECVD $\mathrm{SiO}_{2}$ layer. Following the deposition steps the films are annealed at $1100^{\circ} \mathrm{C}$. The detailed fabrication process flow is given in appendix (A.3).

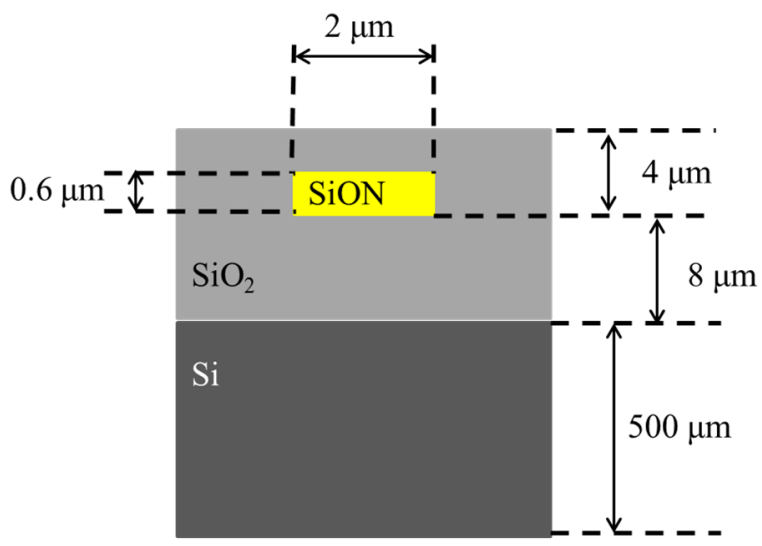

Fig. 3.2.1. Schematic cross-sectional view of the waveguide structure.

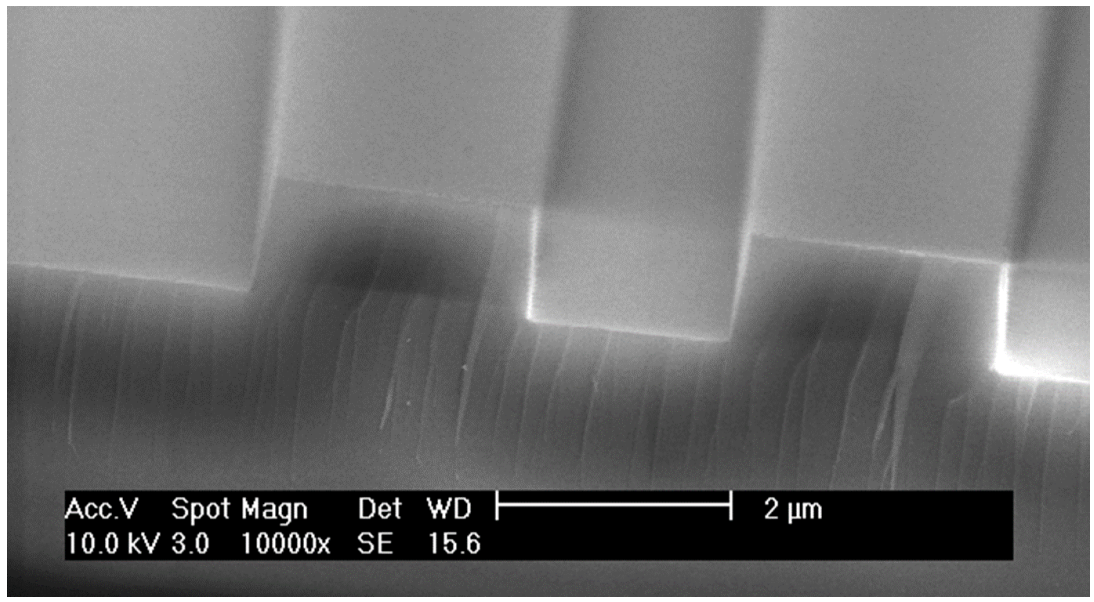

Fig. 3.2.2. SEM image of the fabricated $\mathrm{SiON}$ channel waveguides before the growth of the top cladding. 


\subsection{Adiabatic bend structure design}

Bending waveguide structures are essential components in our OCT system to redirect the propagation direction of light. In practice, unguided stray light, due to the imperfect light coupling between the input fiber and the chip, co-propagates with the guided light in the same direction. The stray light may also be incident on the sample, reflected and coupled into the OCT detection waveguide, thus interfering with the intended reflections. This unwanted interference may reduce the OCT image quality. One of the solutions is using a 90-degree bend after the input waveguide to redirect the guided light to a direction, which does not have unguided stray light.

There are two loss mechanisms associated with waveguide bends [13]. The first mechanism is the transition loss between straight and bent waveguides due to the mismatch of the modal field distributions in those waveguides. Compared to a straight waveguide mode, a bend waveguide mode has a different field distribution which is radially shifted outward. The second mechanism is the intrinsic radiation loss (pure bending loss) of the bend mode. Losses of both mechanisms increase with decreasing bend radii [13].

The aim is to design a bend structure that has a total loss similar to that of a straight waveguide with the same length. Since the bend structure is only used to isolate the unwanted stray light, it is preferred to simply consider a bend as a piece of straight waveguide when analyzing the total losses in an OCT system. This means that the strongly wavelengthdependent bending losses [14] should be small compared to the propagation losses of a straight waveguide (which is in the order of $0.1 \mathrm{~dB} / \mathrm{cm}$ [8]). Calculations of the expected fundamental modes bending losses were performed with the Lumerical finite-difference eigenmode solver [https://www.lumerical.com/]. A non-uniform cross-sectional calculation grid was used for computational efficiency and accuracy. The grid size used in the bending loss calculation is $40 \mathrm{~nm} \times 40 \mathrm{~nm}$ within a (waveguide width +1$) \mu \mathrm{m} \times$ (waveguide height $+1.6) \mu \mathrm{m}$ region around the core and gradually increased to $200 \mathrm{~nm} \times 200 \mathrm{~nm}$ in the rest of the $24 \mu \mathrm{m} \times 24 \mu \mathrm{m}$ simulation window as shown in Fig. 3.3.1. The bending losses increase with wavelength [14]. Therefore, calculations have been made at the highest wavelength $(1640 \mathrm{~nm})$ in the spectral region of interest.

The calculated fundamental mode bending losses for both TE and TM polarizations are presented in Fig. 3.3.2, showing that the loss decreases with increasing bend radius and waveguide width. It can be seen that a $2-\mu \mathrm{m}$ wide waveguide would need a bending radius of at least $1700 \mu \mathrm{m}$ to keep the pure bending losses below $0.1 \mathrm{~dB} / \mathrm{cm}$ for both TE and TM modes. With the same loss requirement, the bend radius can be reduced to $800 \mu \mathrm{m}$ or $700 \mu \mathrm{m}$ in the case of respectively $4-\mu \mathrm{m}$ or $6-\mu \mathrm{m}$ wide bends. The bending waveguide mode evolves into a whispering gallery mode (WGM) by further increasing the bending waveguide width. If the width of the waveguide is large enough, the WGM mode does not reach the inner 
boundary of the bend thus the pure bending losses are not further reduced by increasing the width of the waveguide [15].

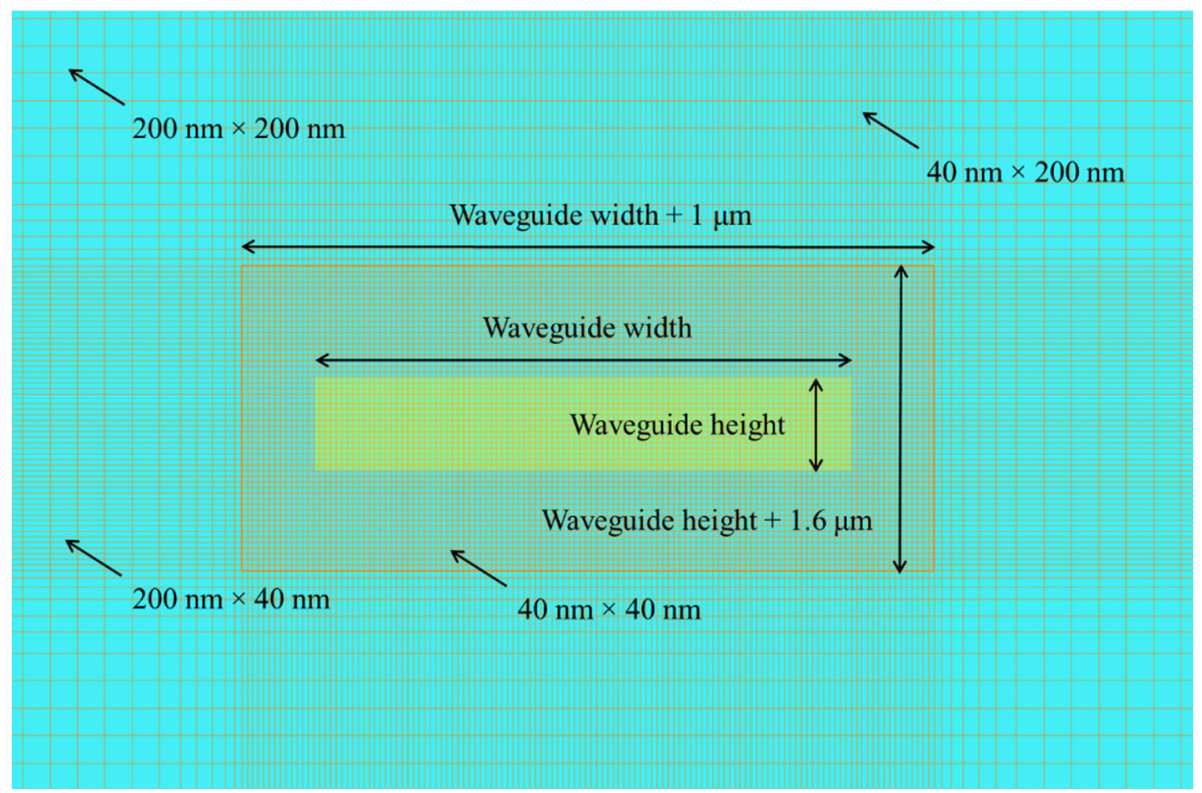

Fig. 3.3.1. Schematic of the bending mode calculation grid. In order to shown the grid clearly, only the center $8 \mu \mathrm{m} \times 5 \mu \mathrm{m}$ region is drawn in this figure.

The calculations shown in Fig. 3.3.2 hold for waveguides having a constant width and bending radius. It is possible, however, to reduce the needed surface area for a bend by allowing both the width and the radius to vary along the waveguide in an adiabatic approach [13]. Fig. 3.3.3 shows a schematic of a 90 degree adiabatic bend which has both a reduced transition loss and a reduced pure bending loss. First, the bend radius $R(l)$ is gradually reduced from a starting radius $R_{\text {start }}$ to a minimum radius $R_{\min }$, where $l$ is the arc length of the bend. The starting radius can be set so large (such as $3000 \mu \mathrm{m}$ or larger) that the loss due to mode mismatch to the straight waveguide is virtually zero. Second, the pure bending loss of the bend mode is reduced by gradually increasing the width, $W(l)$, of the waveguide.

In the adiabatic bend model used in Roeloffzen's paper [13], the radius $R(l)$ evolves as given by Equation (1) in [13], which is reproduced below:

$$
R(l)=R_{\min } \cdot \exp \left(\frac{1}{\ln \left(\frac{R_{\text {start }}}{R_{\text {min }}}\right)+A_{\text {bend }} \cdot l}\right) .
$$

The range of $l$ used in Equation (3.3.1) is from $l=0$ to $\theta(l)=45^{\circ}$ in our adiabatic bend design. 
The rate of change of the radius $A_{b e n d}=0.04 \mu \mathrm{m}^{-1}$, which is chosen based on [15]. This value for $A_{b e n d}$ is recommended and experimentally verified in [15]. Larger starting radius $R_{\text {start }}=3000 \mu \mathrm{m}$ and minimum radius $R_{\min }=1500 \mu \mathrm{m}$ are used in our design compared with the ones used in [15] to ensure a low pure bending loss. Note, the minimum radius $(1500 \mu \mathrm{m})$ and the starting radius $(3000 \mu \mathrm{m})$ are approximate settings for Equation (3.3.1) not the exact results.

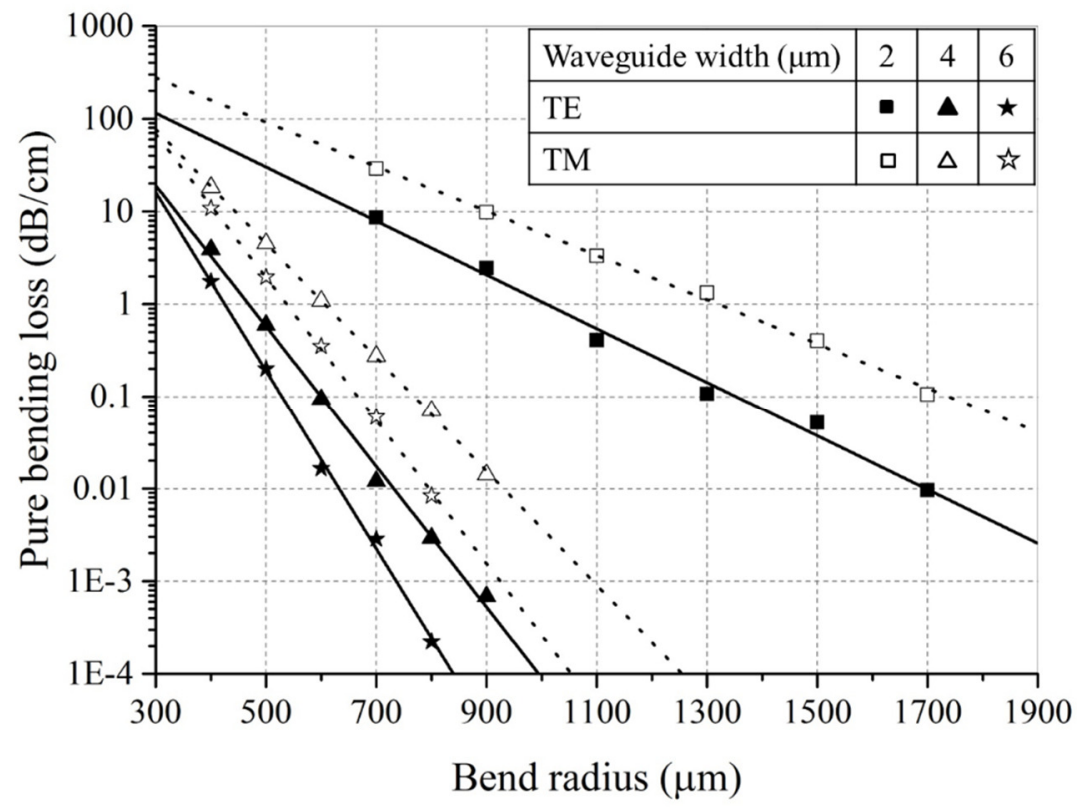

Fig. 3.3.2. Calculated pure bending loss of the fundamental mode as a function of the bend radius, for three different waveguide widths: $2 \mu \mathrm{m}$ (same width as a straight waveguide), $4 \mu \mathrm{m}$ and $6 \mu \mathrm{m}$, respectively. TE modes are represented by solid symbols and TM modes by open symbols. The solid and dotted lines are linear fittings of the calculated loss values for TE and TM modes, respectively.

The width, $W(l)$, of the adiabatic bend is increased linearly from $2 \mu \mathrm{m}$ (at the beginning of the bend) to $6 \mu \mathrm{m}$ (at the minimum bending radius). The waveguide becomes multi-mode for a waveguide width larger than $\sim 2.4 \mu \mathrm{m}$ at $\lambda=1200 \mathrm{~nm}$ and $\sim 4.5 \mu \mathrm{m}$ at $\lambda=1640 \mathrm{~nm}$. However the adiabatic waveguide widening ensures that only the fundamental mode is excited in the multi-mode waveguide bend.

In case of a $90^{\circ}$ bend (Fig. 3.3.3), starting from $\theta=0^{\circ}$, the bending radius decreases to its minimum value while the width of the bending waveguide increases to its maximum value at $\theta=45^{\circ}$; from $45^{\circ}$ to $90^{\circ}$, the changes of bending radius and waveguide width are reversed. 


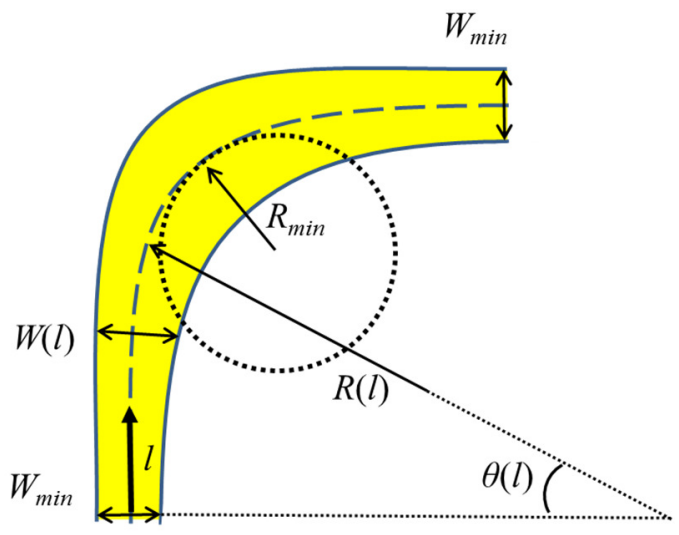

Fig. 3.3.3. Schematic of the adiabatic bend.

The total loss of this adiabatic bend structure is not calculated since it is expected to be dominated by the waveguide propagation loss. This expectation is based on the following facts. This structure can be considered as many short bend sections with different widths and bending radii connected together. First, based on the calculations in Fig. 3.3.2, every section in this adiabatic bend has a pure bending loss much smaller than the propagation loss $(0.1 \mathrm{~dB} / \mathrm{cm})$. Thus the pure bending loss of the whole structure is negligible compared to the propagation loss. Second, based on the work in [15], the chosen value for $A_{\text {bend }}$ and the rate with which the waveguide width changes over distance is small enough to have negligible total transition loss between all sections compared with the propagation loss [15].

We did not have exact total loss measurement of the fabricated adiabatic bend, since it is not a primary goal of our study. (Note, in order to measure the loss of such a low-loss adiabatic bend, few tens to few hundreds of cascade bends are needed. It is time consuming to design a proper structure and perform such loss measurements.) However, based on all the experiments (in Chapter 3, Chapter 4 and Chapter 5) we have performed, there is no evidence that this adiabatic bend has more loss than the straight waveguide in the wavelength range of interest (1200 nm - $1640 \mathrm{~nm})$. Therefore, this adiabatic bend design fulfills the design criteria.

\subsection{Y junction design and characterization}

The Y junction is one of the key components in the common-path OCT system. It is used for connecting the light source, the detector, and the common-path arm (the sample-reference arm). Low losses and correct splitting ratios of the Y junction are very important in achieving a good power efficiency of the OCT system as discussed in Section 2.6. Since we would like to cover two commonly used OCT lasers, centered at $1310 \mathrm{~nm}$ and $1550 \mathrm{~nm}$ respectively 
with the same Y junction, a broad working wavelength range $(1200 \mathrm{~nm}-1640 \mathrm{~nm})$ is preferred.

In theory, the conventional $\mathrm{Y}$ junction perfectly fulfills the requirements mentioned above. However, in practice the conventional Y junction suffers from fabrication errors (will be discussed in detail below). A common solution to reduce the sensitivity to fabrication errors is a blunt $\mathrm{Y}$ junction design. However, the blunt $\mathrm{Y}$ junction shows an increased loss. Some studies have focused on the modification of the blunt $\mathrm{Y}$ junction design to lower their losses [16, 17]. Both of these studies work with a multimode interference (MMI) based Y junction. Wang et al. presented a tapered MMI based Y junction which had a simulated loss as low as $0.015 \mathrm{~dB}(0.36 \%)$ at a wavelength of $1550 \mathrm{~nm}$ [16]. However, the reported operating wavelength range is from $1500 \mathrm{~nm}$ to $1600 \mathrm{~nm}$ only.

In order to make a $\mathrm{Y}$ junction working in a wavelength range as broad as possible, we propose a different approach where only the fundamental mode is considered. The property and limitations of conventional $\mathrm{Y}$ junction and a normal blunt $\mathrm{Y}$ junction are introduced below. This is used as a reference to discuss how we modified the blunt $\mathrm{Y}$ junction in Section 3.4.1.

Conventional $\mathrm{Y}$ junctions have very large working wavelength range achieved by splitting the light geometrically. A schematic of the conventional Y junction is shown in Fig. 3.4.1(a). Three straight waveguides (with width $W$ ) are connected together, where the ports 2 and 3 are located axisymmetrically with respect to the waveguide of port 1 . The typical angle $\theta$ between ports 2 and 3 should be in the order of $2^{\circ}$ or smaller to minimize the losses [18]. With this geometrical splitting, the power input to port 1 is evenly divided over ports 2 and 3, as long as all waveguides are single mode.

However, in order to act as an ideal, lossless 50/50 splitter, this type of Y junction requires an infinitely sharp vertex at $z_{c 2}$. Such a sharp feature cannot be fabricated due to the finite fabrication resolution. A finite size tip will introduce losses to the $\mathrm{Y}$ junction, and random fabrication errors may cause a deviation of the desired 50/50 splitting ratio. This leads to a conclusion that the limitations of the fabrication process should be taken into account, leading to a design with a well-defined 'blunt'-shaped waveguide joint as shown in Fig. 3.4.1(b). However, the finite gap between ports 2 and 3 leads to unavoidable optical losses. The loss is positively correlated with the gap size $G$ and the minimal reproducible gap size depends on the fabrication technology.

In Section 3.4.1, we present a design of a modified blunt $Y$ junction which has a reduced sensitivity to fabrication errors. The $\mathrm{Y}$ junction shape is optimized to reduce the losses without reduce the minimal reproducible gap size. In Section 3.4.2, the reproducibility (in fabrication) of this design is verified by measuring the splitting ratio of many fabricated $\mathrm{Y}$ junctions at wavelengths of $1.31 \mu \mathrm{m}$ and $1.55 \mu \mathrm{m}$. In addition we show measurements of the splitting ratio of the $\mathrm{Y}$ junction as function of the wavelength. The losses of the $\mathrm{Y}$ junction are discussed in Section 3.4.4. 


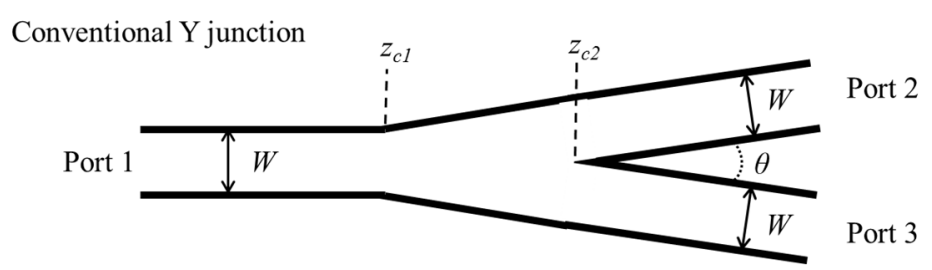

(a)

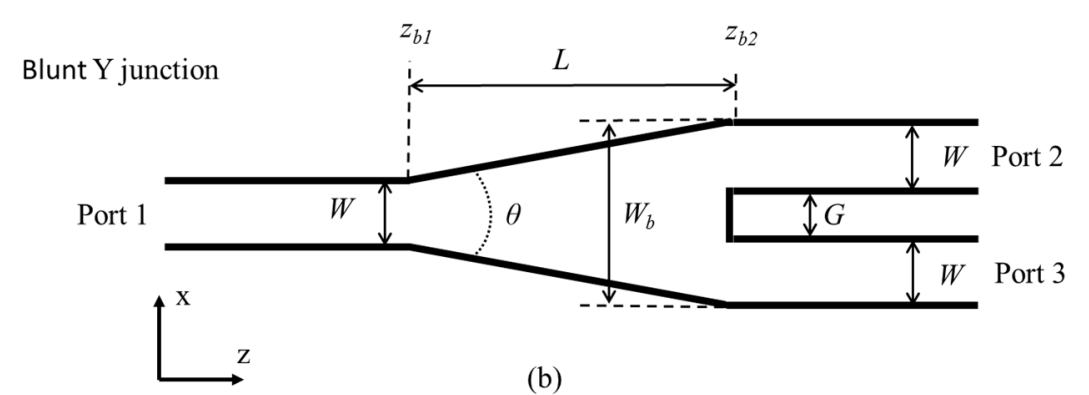

Fig. 3.4.1. (a) Schematic of the conventional $Y$ junction. (b) Schematic of the blunt $Y$ junction.

\subsubsection{Modified blunt $Y$ junction design}

The modified blunt $\mathrm{Y}$ junction has reduced losses compared with the commonly used blunt $\mathrm{Y}$ junction due to the modified transition at the end of tapered section $z b 2$. A schematic layout of the modified $\mathrm{Y}$ junction is shown in Fig. 3.4.2. The main difference compared with Fig. 3.4.1(b) is the taper width at $z b 2$.

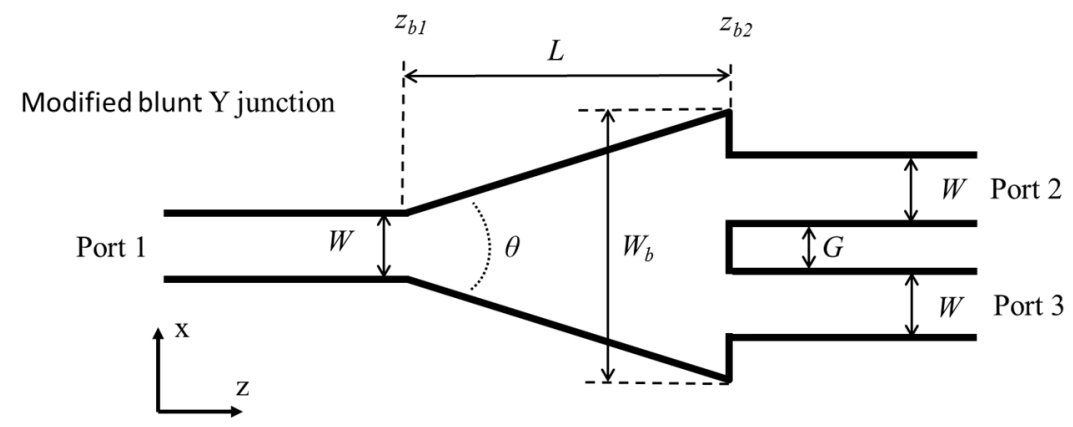

Fig. 3.4.2. Schematic of the modified blunt $Y$ junction. 
The minimum dimensions of gap $G$ between port 2 and port 3 are determined by the finite resolution of the fabrication technology. In case of our SiON waveguide technology, the minimum openable gap is about $500 \mathrm{~nm}$, the minimum reproducible gap is about $800 \mathrm{~nm}$. Therefore $G$ is designed to be $1000 \mathrm{~nm}$ in order to have a sufficiently large tolerance for fabrication errors that may cause changes in waveguide width, and hence gap width. The waveguide width at all the ports is $2 \mu \mathrm{m}$, which is the same as that of all the straight waveguides on the chip. Assume that light is propagating from left to right in Fig. 3.4.2. The input mode at port 1 is to expand adiabatically (i.e without exciting any higher-order or radiation modes) from width $W$ to $W_{\mathrm{b}}$ over a distance $L$ toward the end of the tapered section. A length as large as $L=200 \mu \mathrm{m}$, which is long enough to have the taper angle $\theta$ smaller than $2^{\circ}$, ensures this linear taper is adiabatic.

At the end of the tapered section $z, b 2$ power is coupled from the large fundamental mode in the taper to the two smaller waveguide modes in port 2 and port 3. Losses are caused by the structural discontinuity at the coupling location and the imperfect overlap of the modal field distributions. The mode overlap is expected to be different for different taper widths $W_{b}$. The $\mathrm{Y}$ junction loss as a function of $W_{b}$ has been simulated with a 3-dimentional beam propagation method (BPM) [19]. A grid size of $50 \times 50 \times 300 \mathrm{~nm}^{3}$ (x by y by z) was used within a $26 \times 16 \times 650 \mu \mathrm{m}^{3}$ simulation window. The loss of the $\mathrm{Y}$ junction is determined as

$$
\zeta=\left(P_{p o r t 1}-P_{p o r t 2}-P_{p o r t 3}\right) / P_{p o r t 1} \times 100 \% \text {. }
$$

The simulation at two wavelengths, $1310 \mathrm{~nm}$ and $1550 \mathrm{~nm}$ for both TE and TM modes are shown in Fig 3.4.3. The simulation at $W_{b}=5 \mu \mathrm{m}$ is the same as a normal blunt $\mathrm{Y}$ junction shown in Fig. 3.4.1(b). For both wavelengths the minimum loss is found for $W_{b}=6.8 \mu \mathrm{m}$. The loss at $1550 \mathrm{~nm}$ is lower than at $1310 \mathrm{~nm}$. This is due to the fact that the longer the wavelength the more the mode extends outside the waveguide core, effectively filling up the gap between ports 2 and 3, thus providing better matching of the modal fields.

This $\mathrm{Y}$ junction is a reciprocal optical component, implying that the transmission between each pair of ports is independent of the direction of wave propagation. Consider, for example, the case of light with a wavelength of $1310 \mathrm{~nm}$ entering the $\mathrm{Y}$ junction (with $W_{b}=6.8 \mu \mathrm{m}$ ) from port 1 . The loss is $8 \%$ and the remaining power is equally distributed over ports 2 and 3, each one receiving $46 \%$ of the input power. This indicates that $46 \%$ of the power in the fundamental mode of the tapered section is coupled into the fundamental mode of port 2. If the propagation direction is reversed, $46 \%$ of the power entering in the fundamental mode through port 2 couples into the fundamental mode of the tapered section, which is adiabatically coupled into the fundamental mode in port 1 . Thus the light coupling efficiency from port 2 (or port 3 ) to port 1 is $46 \%$. The rest of the light is coupled into higher order modes or radiation modes of the tapered section. None of these modes are supported in the single mode waveguide at port 1 . The general case of input into both port 2 and port 3 
with different power and phase does not apply to our OCT system, and is therefore outside the scope of this analysis.

This simulation indicates that the optimal choice of $W_{b}=6.8 \mu \mathrm{m}$ is not very critical. In the range $W_{b}=6.3-7.3 \mu \mathrm{m}$ the $\mathrm{Y}$ junction has almost identical minimum loss. This range is well within the typical fabrication error, which is $\pm 0.1 \mu \mathrm{m}$. This design leads to a loss reduction of almost 50\% compared to a normal blunt $\mathrm{Y}$ junction $\left(W_{b}=5 \mu \mathrm{m}\right.$, in Fig. 3.4.3). The remaining few percent loss in the $\mathrm{Y}$ junction only results in less than $1 \mathrm{~dB}$ reduction in the OCT signal-to-noise ratio (SNR) and is acceptable as discussed in Section 2.6.

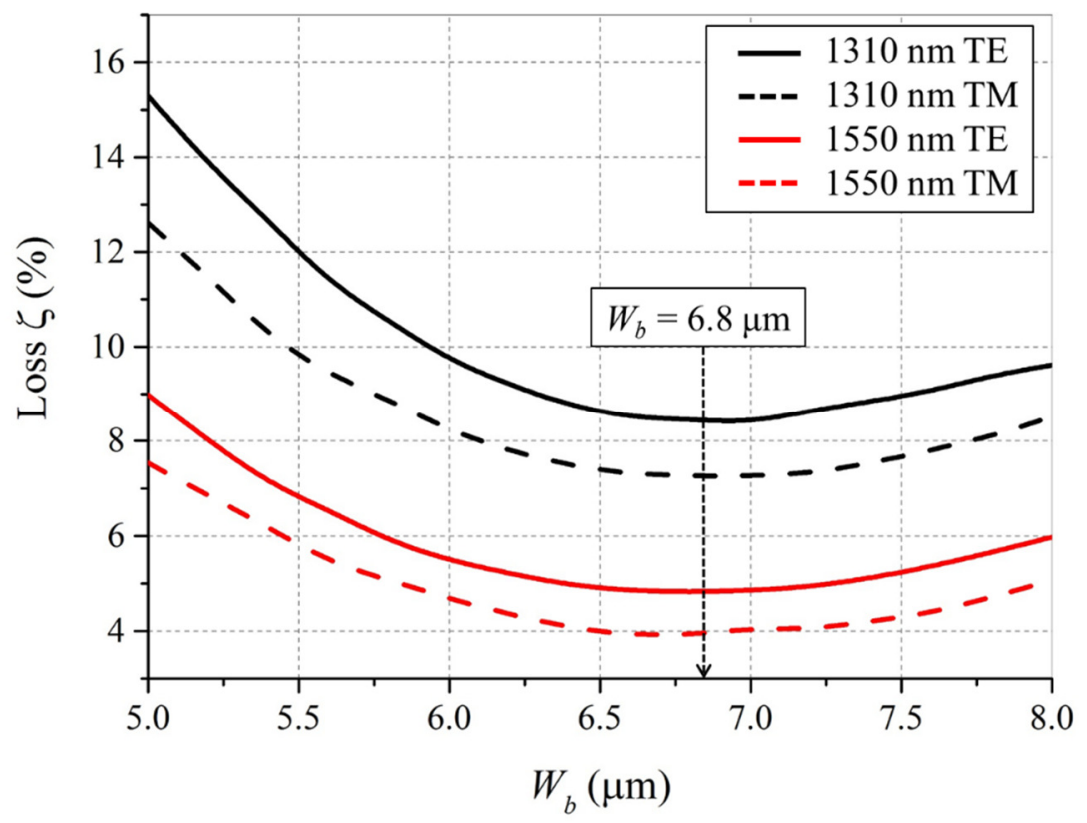

Fig. 3.4.3. Calculated loss of the $Y$ junction using $W=2 \mu \mathrm{m}, L=200 \mu \mathrm{m}$ and $G=1 \mu \mathrm{m}$. The loss of the $\mathrm{Y}$ junctions is minimum for both wavelengths of $1310 \mathrm{~nm}$ and $1550 \mathrm{~nm}$ when the taper width $W_{b}$ is $6.8 \pm 0.5 \mu \mathrm{m}$.

\subsubsection{Fabrication result of the $Y$ junction}

In order to examine the fabrication result, a microscope image of a fabricated $\mathrm{Y}$ junction (before the growth of the top cladding) is shown in Fig 3.4.4. The finite fabrication resolution leads to the rounded edges at the gap tip and at the corners at the end of the tapered section. These rounded corners may lead to a smoother transition with a slightly lower loss. The effects of these subwavelength $(<1 \mu \mathrm{m})$ features may be simulated with finite-difference time-domain (FDTD) method [20] in the future if more precise simulations are needed. 


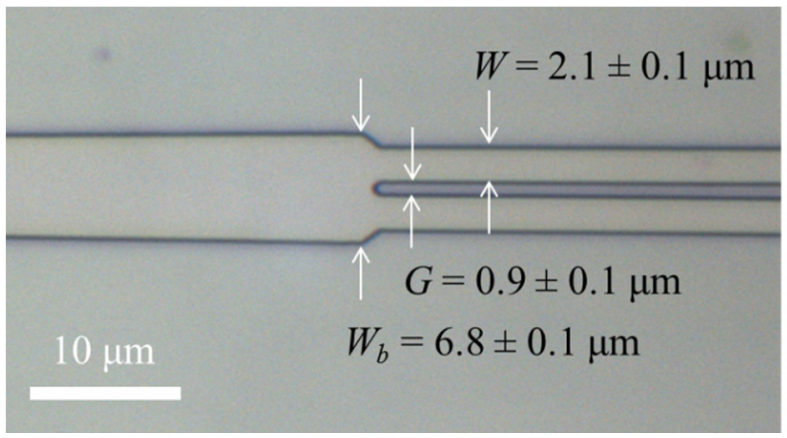

Fig. 3.4.4. A microscope image of a fabricated $Y$ junction before the growth of the top cladding. Port 2 , port 3 and part of the tapered section is shown in this image. The finite fabrication resolution leads to the rounded edges at the gap tip and at the corners at the end of the tapered section.

\subsubsection{Y junction splitting ratio measurements}

In order to verify the reproducibility of the modified Y junction design, the measured splitting ratios of many fabricated $\mathrm{Y}$ junctions are discussed in this section.

Figure 3.4.5(a) shows a schematic of the experimental setup applied to a 1-to-4 power splitter as an example. The chip layout and the setup are designed to minimize the power measurement uncertainty introduced by variations in the efficiency of coupling light to and from the chip, and possible stray light caused by incomplete coupling. By including a 90-degree bend in the input waveguide, the stray light (the light from the input fiber which is not coupled into the guided waveguide mode) is prevented from reaching the photodetector. Second, the coupling efficiency from the output ports to the detector is maximized and made insensitive to small misalignments by using a large-area detector without any intervening optical elements.

Examples of typical measurements are shown in Fig. 3.4.5(b) and Fig. 3.4.5(c). The power values $P_{n}$ at the (nearly $100-\mu \mathrm{m}$ wide) flat tops of the measured peaks correspond to the output power of the respective ports. The power ratio of port 2 (Fig. 3.4.2) of $n^{\text {th }} Y$ junction is defined as

$$
\gamma_{n 2}=\frac{P_{p o r t 2}}{P_{p o r t 2}+P_{p o r t 3}} .
$$




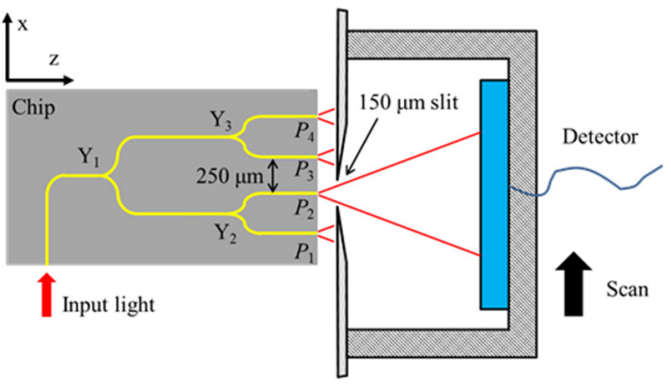

(a)

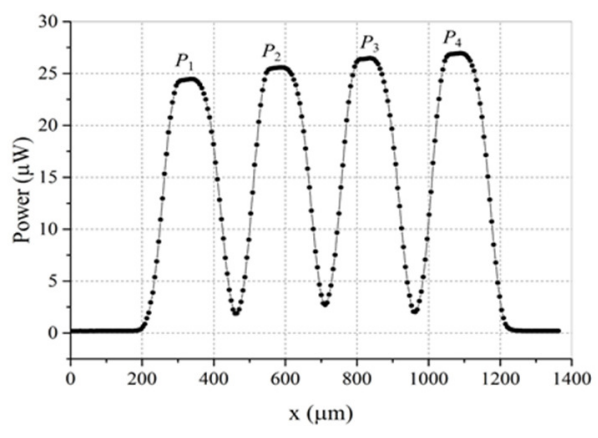

(b)

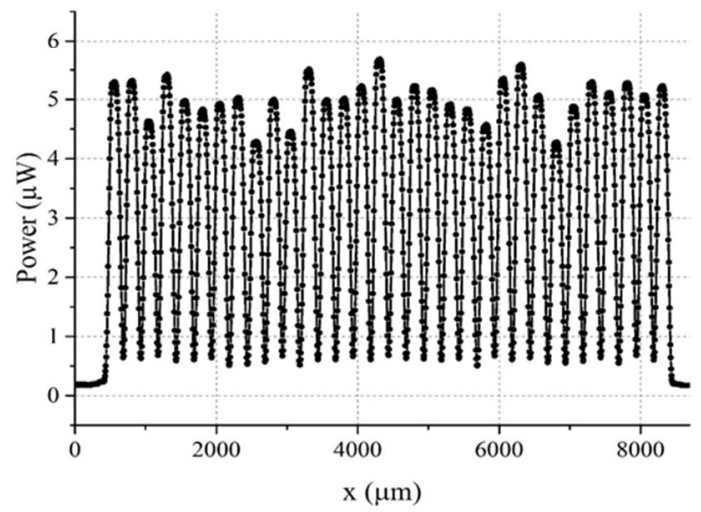

(c)

Fig. 3.4.5. Y junction splitting ratio measurement setup and results. (a) Schematic of the experimental setup. The input light is launched from a standard single mode telecommunication fiber with a $9-\mu \mathrm{m}$ core. The 90-degree bend before junction $Y_{1}$ is an adiabatic bend as described in Section 3.3. The output light passes through a $150-\mu \mathrm{m}$ wide slit and detected with a large area detector (larger than the beam size on the detector). The output ports spacing is $250 \mu \mathrm{m}$ and the slit, located at a few tens of micrometers distance from the chip facet, is used to block the light from all channels except the one to be measured. The slit + detector assembly is moved parallel to the $\mathrm{x}$-axis to scan the power from the output ports one by one. (b) The raw data of a 1-to-4 splitter sample measured at a wavelength of $1306 \mathrm{~nm}$. (c) The raw data of a 1-to-32 splitter sample measured at a wavelength of $1306 \mathrm{~nm}$.

There are three $\mathrm{Y}$ junctions in this 1-to-4 splitter. Power ratio $\gamma_{n 2}$ of junction $Y_{n}$ can be calculated directly from the measured output powers as: $\gamma_{12}=\left(P_{3}+P_{4}\right) /\left(P_{1}+P_{2}+P_{3}+P_{4}\right)$; $\gamma_{22}=P_{2} /\left(P_{1}+P_{2}\right)$ and $\gamma_{32}=P_{4} /\left(P_{3}+P_{4}\right)$. These equations hold for the case in which the loss for each $\mathrm{Y}$ junction is the same. Figure 3.4.6 shows a test structure of five 1-to-N power splitters comprising $57 \mathrm{Y}$ junctions. The splitting ratio of every $\mathrm{Y}$ junction has been measured with the method mentioned above. 


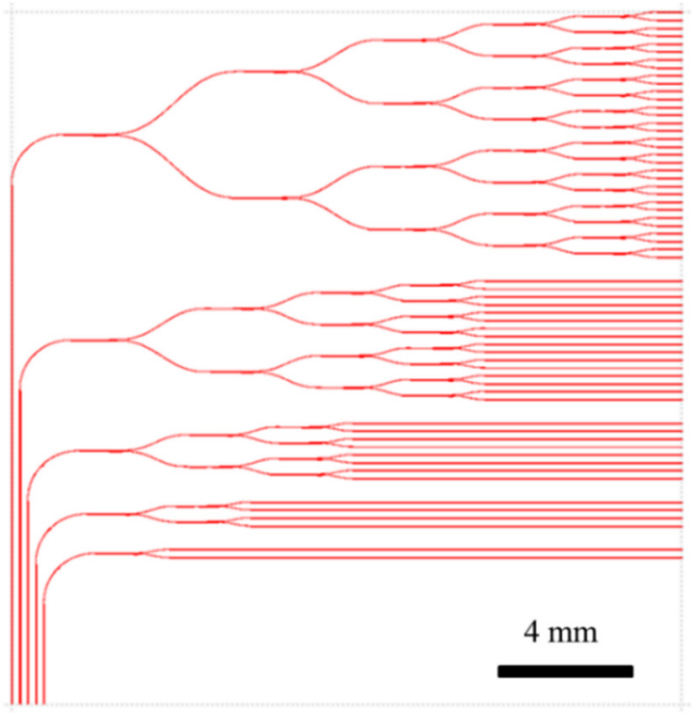

Fig. 3.4.6. Layout of the test structure for measuring the splitting ratio of $Y$ junctions.

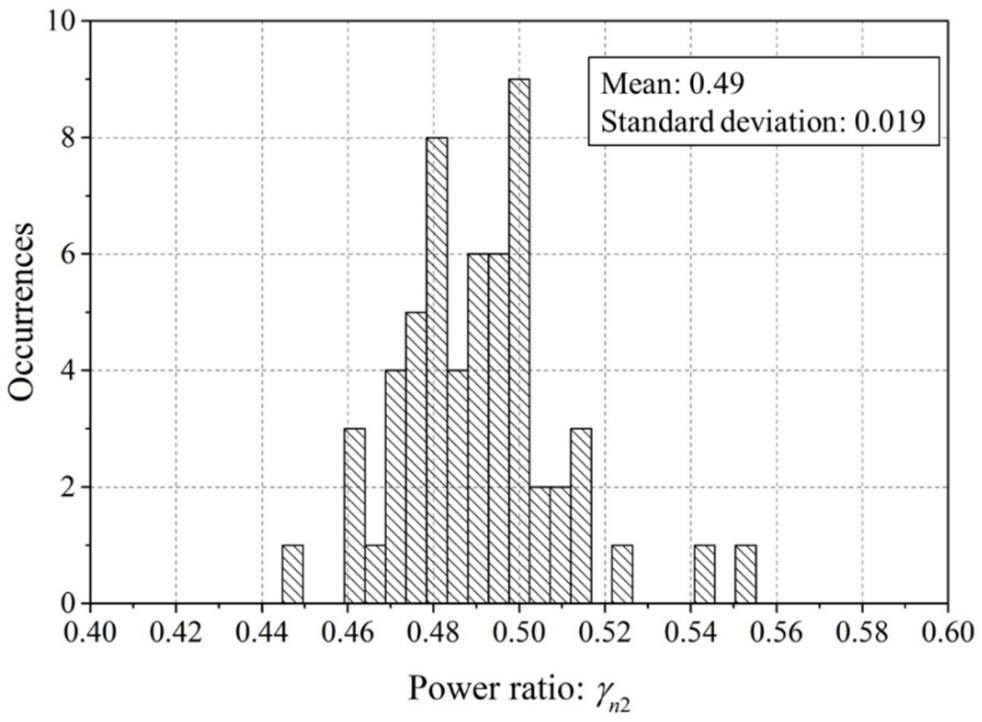

Fig. 3.4.7. Histogram of measured power ratios of $57 \mathrm{Y}$ junctions.

A histogram of all measured power ratios $\gamma_{n 2}$ at a wavelength of $1306 \mathrm{~nm}$ (using a singlewavelength laser source) is shown in Fig 3.4.7. The mean value of the power ratio $\gamma_{n 2}$ is 0.49 with a standard deviation of 0.019 . Thus the mean value of the power ratio $\gamma_{n 3}$ is $1-0.49=0.51$ with a standard deviation of 0.019 . (The power measurement error is very small and has a negligible contribution to this standard deviation.) These results indicate that the fabrication 
tolerance of our modified $\mathrm{Y}$ junction design satisfies the requirements discussed in Section 2.6.

Power ratio measurements have been repeated for a wavelength of $1544 \mathrm{~nm}$. The measured mean value of the power ratio $\gamma_{n 2}$ is also 0.49 with a standard deviation of 0.019 . For a given $\mathrm{Y}$ junction, the power ratio different at wavelength $1306 \mathrm{~nm}$ and $1544 \mathrm{~nm}$ is an indication of the wavelength dependency of this particular $\mathrm{Y}$ junction. The power ratio difference of $\gamma_{n 2}(1306 \mathrm{~nm})-\gamma_{n 2}(1544 \mathrm{~nm})$ for each Y junction is shown in the histogram of Fig. 3.4.8. Difference of zero means an identical power splitting ratio for both wavelength. In Fig. 3.4.8, the averaged power ratio difference is as low as $-8 \times 10^{-4}$, and the standard deviation is 0.01 . This result indicate a nearly identical power splitting ratio at wavelength of $1306 \mathrm{~nm}$ and $1544 \mathrm{~nm}$ for every measured Y junction.

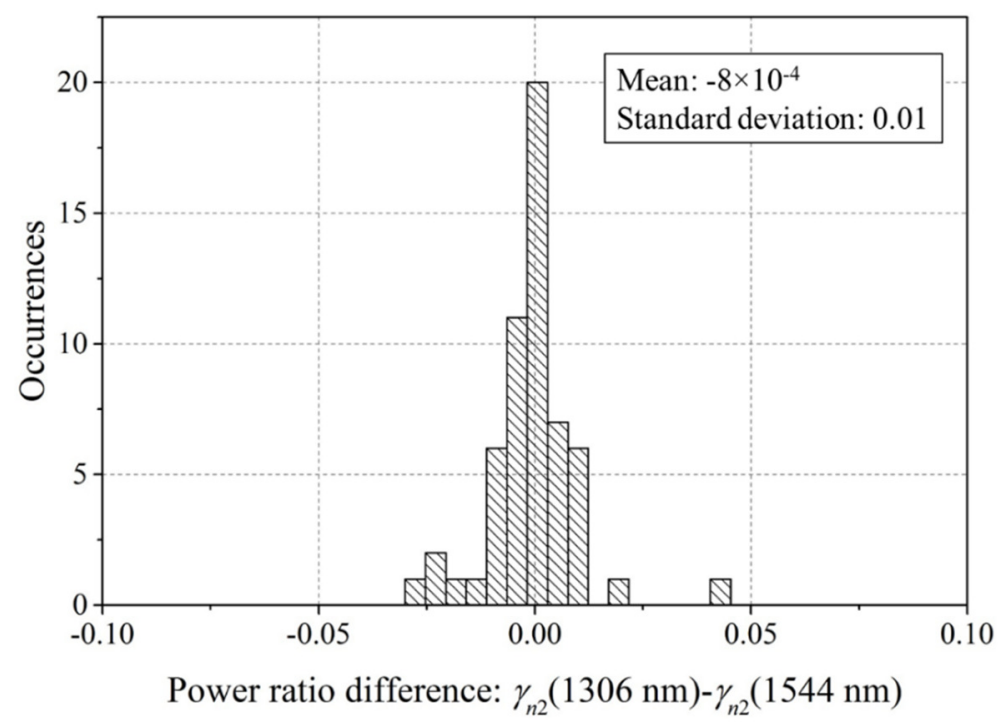

Fig. 3.4.8. Histogram of the measured power ratio difference in $Y$ junctions at the wavelength of $1306 \mathrm{~nm}$ and $1544 \mathrm{~nm}$.

Next, the power ratio of a single $\mathrm{Y}$ junction as a function of wavelength is measured. For this we used a broadband light source (Fianium SC-450-2, $460 \mathrm{~nm} \sim 2000 \mathrm{~nm}$ wavelength range) and a spectrometer (HORIBA iHR550 with an InGaAs detector). Unlike the setup described in Fig. 3.4.5, the output ports have to be fiber coupled to the spectrometer due to practical limitations. Thus, two standard $9-\mu \mathrm{m}$ core single mode fibers are used to couple the light from the light source to the chip (port 1 in Fig. 3.4.2) and from the chip (port 2 or port 3) to the spectrometer. Since the uncertainty of fiber-chip-fiber coupling efficiency (caused by alignment uncertainty) has a considerable influence on the spectral magnitude, the spectra measured from port 2 and port 3 are not directly comparable. However, based on our experience, the changes of the coupling efficiency, due to alignment uncertainty ( $\pm 0.3 \mu \mathrm{m}$ in 
the transverse direction; $\pm 10 \mu \mathrm{m}$ in the light propagation direction), is almost wavelength independent. This may due to the fact that the fiber mode (in the order of $10 \mu \mathrm{m}$ ) is much larger than the waveguide mode (in the order of $4 \mu \mathrm{m}$ ). The power ratio at wavelength of $1306 \mathrm{~nm}$ has already been measured accurately with the slit-plus-power-meter setup mentioned in Fig. 3.4.5(a). Thus, the fiber-chip-fiber coupling efficiency uncertainty is eliminated by rescaling the measured spectra with a single factor that, when multiplied to the spectrum of port 2, equates the power measured at $1306 \mathrm{~nm}$ with the broadband source to that measured with the single-wavelength source in the setup of Fig. 3.4.5(a). The same operation was also performed for port 3.

The rescaled spectra of port $2 P_{2}(\lambda)$ and port $3 P_{3}(\lambda)$ are shown in Fig 3.4.9. These spectra are used to calculate the power ratio $\gamma_{2}(\lambda)=P_{2}(\lambda) /\left(P_{2}(\lambda)+P_{3}(\lambda)\right)$ of port 2 , which is also presented in Fig. 3.4.9. The measured power ratio $\gamma_{2}(\lambda)$ has a mean value of 0.506 with a standard deviation of 0.011 between $1170 \mathrm{~nm}$ and $1650 \mathrm{~nm}$.

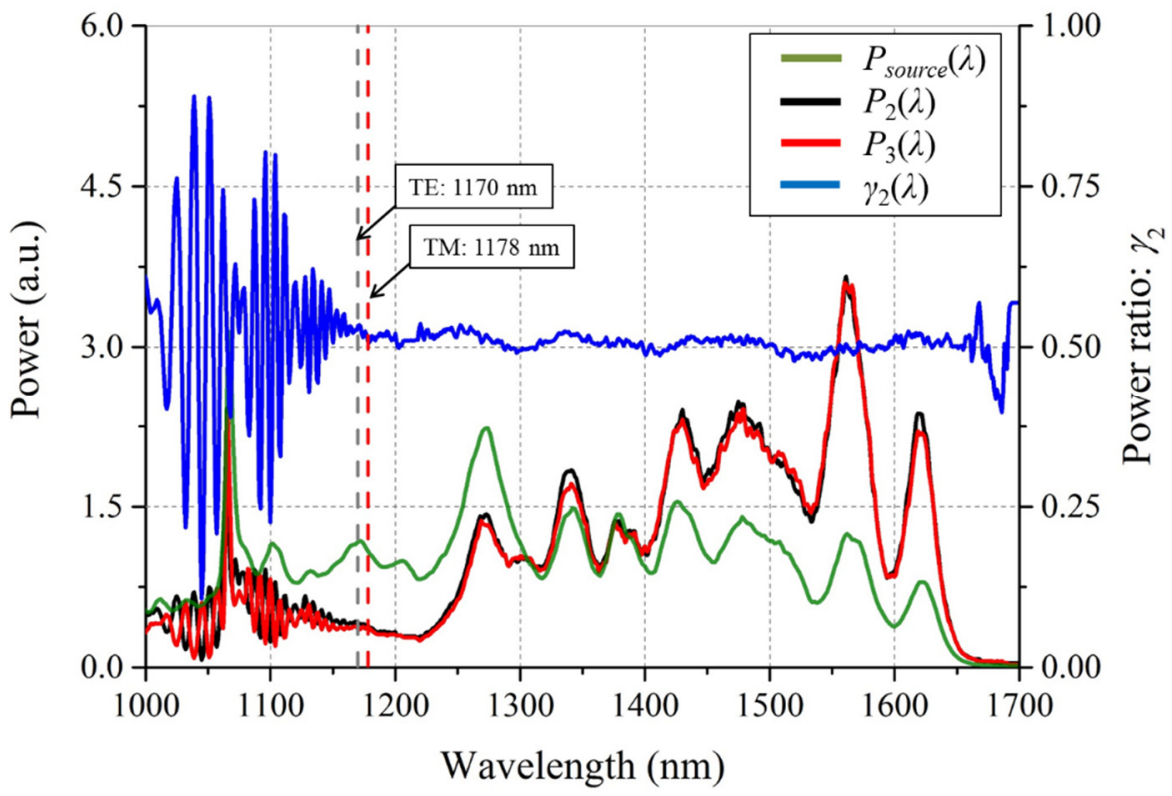

Fig. 3.4.9. Wavelength dependence of the power ratio. The measured spectrum of the light source $P_{\text {source }}(\lambda)$ is rescaled arbitrarily to properly display in this plot. The measured spectra of port $2 P_{2}(\lambda)$ and port $3 P_{3}(\lambda)$ are rescaled to have a power ratio of 0.497 at the wavelength of $1306 \mathrm{~nm}$ as measured with the setup mentioned in Fig. 3.4.5(a). The power splitting ratio $\gamma_{2}(\lambda)$ is calculated based on these two spectra. The input and output waveguides are single mode for TE and TM polarization at wavelengths longer than $1170 \mathrm{~nm}$ and $1178 \mathrm{~nm}$, respectively.

The large fluctuation of the power ratio observed at wavelengths shorter than $1170 \mathrm{~nm}$ is caused by the multimode behavior of the input and output waveguides. Calculations show that the waveguide becomes multi-modal for TE-polarization at wavelengths shorter than 
$1170 \mathrm{~nm}$, and for TM at wavelengths shorter than $1178 \mathrm{~nm}$. The power ratio was measured with unpolarized light and the starting point of the strong short-wavelength fluctuations agrees well with the calculated onset of multi-modality.

The large power ratio fluctuations observed at wavelengths larger than $1650 \mathrm{~nm}$ are caused by the strong increase of measurement noise, due to the decreasing sensitivity of the InGaAs detector at these wavelengths. There is no reason to expect the splitting ratio to exhibit strong fluctuations at wavelengths beyond $1650 \mathrm{~nm}$.

The common power fluctuation in both port 2 and port 3 at wavelengths longer than $1170 \mathrm{~nm}$ originates outside the chip. This is confirmed by a measurement of the spectrum of the light source $P_{\text {source }}(\lambda)$. This source spectrum is modified by the presence of several optical elements (lenses and $950 \mathrm{~nm}$ long pass filter NT66-238 from Edmund Optics). Since the splitting ratio measurement is a relative measurement, the source spectral fluctuations are cancelled out. Due to the better fiber-chip coupling efficiency (better mode overlap between the waveguide mode and fiber mode) and lower propagation loss at the longer wavelengths, the amplitude ratio between the spectrum of port 2 (or port 3) and light source is also larger at longer wavelength.

The results discussed above show that the modified blunt $\mathrm{Y}$ junction fulfills the requirements of our OCT application mentioned in Section 1.2.2 and Section 2.6. The modified $\mathrm{Y}$ junction has enough tolerance to the typical fabrication errors in our SiON waveguide technology as shown in Fig. 3.4.7. The measured wavelength-independent equalpower splitting range is $1170 \mathrm{~nm} \sim 1650 \mathrm{~nm}$, which is limited by the InGaAs detector sensitivity at the long wavelength side. The real upper limit of the wavelength-independent equal-power splitting range is expected to be the long-wavelength edge of the SiON transparency window (about $2.5 \mu \mathrm{m}$ ). Since the OCT axial resolution is inversely proportional to the wavelength bandwidth, such a wide wavelength-independent region is very attractive for ultra-high resolution OCT systems.

\subsubsection{Losses of the $Y$ junction}

The structure shown in Fig 3.4.10 has been designed for measuring the loss of the Y junctions. With an input power $P_{\mathrm{in}}$, a power ratio of $\gamma$, and overall transmittance of the junction $T_{\mathrm{Y}}$, the expected output $P_{\mathrm{n}}$ after $n$ cascaded Y junctions is calculated as

$$
P_{n}=P_{i n} \cdot\left(\gamma T_{Y}\right)^{n} T_{\text {waveguide } \_n} .
$$

$T_{\text {waveguide } \_n}$ is the transmittance of the waveguide from the input to the $n^{\text {th }}$ output. The transmittance is less than 1 due to the propagation loss $(0.1 \mathrm{~dB} / \mathrm{cm}[8])$ of the waveguide. However, since the total length of the test structure is $17 \mathrm{~mm}$ and the length difference between each path are only in the order of $20 \mu \mathrm{m}$ to $100 \mu \mathrm{m}$. $T_{\text {waveguide } \_n}$ can be considered to 
be the same for every path. $P_{\mathrm{n}}$ is measured with the setup described in Fig. 3.4 .5 (no control of the polarization). By plotting $\log _{10}\left(P_{\mathrm{n}}\right)$ against $n$, as shown in Fig. 3.4.11, we expect a straight line with a slope equal to $\log _{10}\left(\gamma T_{\mathrm{Y}}\right)$. So by fitting the data points with a linear function, the value of $\gamma T_{\mathrm{Y}}$ is determined as $\gamma T_{\mathrm{Y}}(1306 \mathrm{~nm})=0.483 \pm 0.003$ and $\gamma T_{\mathrm{Y}}(1544 \mathrm{~nm})=0.499 \pm 0.003$ respectively.

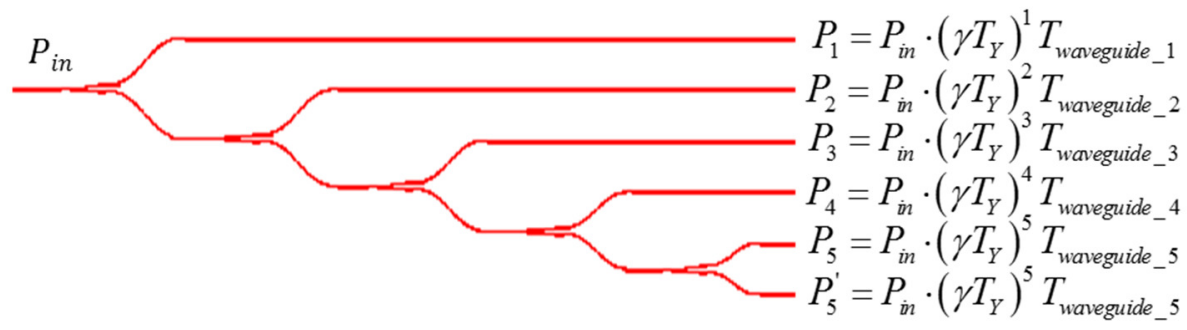

Fig. 3.4.10. Test structure layout and the model used to measure the losses of the Y junction.

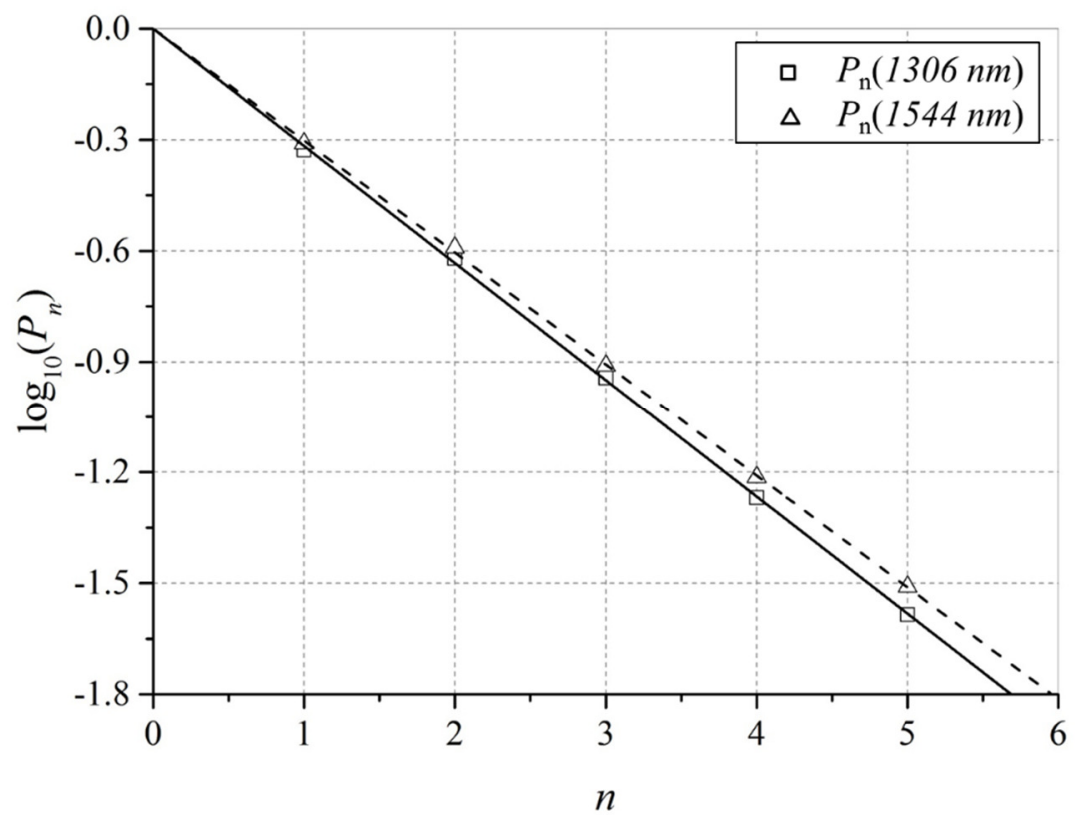

Fig. 3.4.11. $Y$ junction losses measurements. The $x$ axis is the number of $Y$ junction path through. The power measurement error bar of each data point is too small to be seen in this plot. The lines are the corresponding linear fitting at wavelength of $1306 \mathrm{~nm}$ and $1544 \mathrm{~nm}$. The output power of each port $P_{n}$ is normalized to $P_{\text {in }} \cdot T_{\text {waveguide } n} n=1$ (unit less) by shifting their linear fitting intercept to 0 . The fitting slopes are not affected, resulting $\gamma T_{\mathrm{Y}}(1306 \mathrm{~nm})=0.483 \pm 0.003$ and $\gamma T_{\mathrm{Y}}(1544 \mathrm{~nm})=0.499 \pm 0.003$ respectively. 
However, this model is not complete due to the presence of a small fluctuation in the power ratio $\gamma$, where the measured values are $0.49 \pm 0.019$ and $0.51 \pm 0.019$ for port 2 and port 3 (port number in Fig. 3.4.2), respectively. Therefore, measurements on this structure only allow an upper limit of the loss (the minimal transmittance $T_{\mathrm{Y}}$ ) to be determined approximately. For example, the minimal transmittance $T_{\mathrm{Y}}$ at $1306 \mathrm{~nm}$ is determined by taking $\gamma T_{\mathrm{Y}}(1306 \mathrm{~nm})=0.483-0.003=0.48$ and $\gamma=0.51+0.019=0.529$, thus $T_{\mathrm{Y}}(1306 \mathrm{~nm}) \approx 0.91$. This means the $\mathrm{Y}$ junction has a loss no more than $\sim 9 \%$ at $1306 \mathrm{~nm}$ wavelength. Similarly, the Y junction loss is no more than $\sim 6 \%$ at $1544 \mathrm{~nm}$ wavelength.

The measured loss upper limit agrees well with the simulation shown in Fig. 3.4.3 and satisfies the requirements discussed in Section 1.2.2 and Section 2.6. The lower limit of the $\mathrm{Y}$ junction loss cannot be determined with the method mentioned above since it results in gain instead of loss. In order to be able to more accurately determine the Y junction loss, different models and structures are needed in future work. For example, several independent structures with different number of cascading Y junctions ( 1 to 2,1 to 4,1 to $8, \ldots$ ) and same waveguide length may able to provide a more accurate loss measurement.

\subsection{Summary}

In this chapter the different optical waveguide elements that are required to realize the proposed OCT chip layout are presented. We described the fabrication process of SiON waveguide structures. We discussed the losses in a bend structure and presented an adiabatic bend design to minimize the bending loss. The loss of the adiabatic bend is so small that it can be considered to be equal to that of a straight waveguide with the same length. We presented a modified Y junction, which has enough tolerance to the fabrication error to be used reliably in our OCT system. The Y junction power ratio has been measured to be $0.506 \pm 0.011$ in the wavelength range of $1170 \mathrm{~nm}$ to $1650 \mathrm{~nm}$. The long wavelength end is limited by the detector (InGaAs) but not the Y junction. The expected working range of this Y junction is limited by the material transparency at the long wavelength end, which is about $2.5 \mu \mathrm{m}$.

The losses of all the waveguide components used in our chip are summarized below:

- All the waveguides and bend structures have losses of about $0.1 \mathrm{~dB} / \mathrm{cm}$.

- Connections loss between straight and bend waveguides are negligible.

- Y junction has a loss no more than $\zeta=9 \%$ at $1306 \mathrm{~nm}$ wavelength and no more than $6 \%$ at $1544 \mathrm{~nm}$ wavelength.

These losses influence the OCT SNR differently at different reference-sample power relations and different noise conditions. Different possible combinations are listed in Table 2.6.1. All the components designed in this chapter fulfill the requirements of our OCT system mentioned in Section 1.2.2 and Section 2.6 at all system conditions in Table 2.6.1. 


\section{References}

1. Biedermann, B.R., W. Wieser, C.M. Eigenwillig, and R. Huber, Recent developments in Fourier Domain Mode Locked lasers for optical coherence tomography: Imaging at $1310 \mathrm{~nm}$ vs. $1550 \mathrm{~nm}$ wavelength. Journal of biophotonics, 2009. 2(6-7): p. 357-363.

2. Weiss, N., T.G. van Leeuwen, and J. Kalkman, Localized measurement of longitudinal and transverse flow velocities in colloidal suspensions using optical coherence tomography. Physical Review E, 2013. 88(4): p. 042312.

3. Worhoff, K., L.T.H. Hilderink, A. Driessen, and P.V. Lambeck, Silicon oxynitride - A versatile material for integrated optics applications. Journal of the Electrochemical Society, 2002. 149(8): p. F85-F91.

4. Yurtsever, G., N. Weiss, J. Kalkman, T.G. van Leeuwen, and R. Baets, Ultra-compact silicon photonic integrated interferometer for swept-source optical coherence tomography. Optics Letters, 2014. 39(17): p. 5228-5231.

5. Yurtsever, G., B. Považay, A. Alex, B. Zabihian, W. Drexler, and R. Baets, Photonic integrated MachZehnder interferometer with an on-chip reference arm for optical coherence tomography. Biomedical Optics Express, 2014. 5(4): p. 1050-1061.

6. Akca, B.I., B. Považay, A. Alex, K. Wörhoff, R.M. de Ridder, W. Drexler, and M. Pollnau, Miniature spectrometer and beam splitter for an optical coherence tomography on a silicon chip. Optics Express, 2013. 21(14): p. 16648-16656.

7. Culemann, D., A. Knuettel, and E. Voges, Integrated optical sensor in glass for optical coherence tomography (OCT). Ieee Journal of Selected Topics in Quantum Electronics, 2000. 6(5): p. 730-734.

8. Germann, R., H. Salemink, R. Beyeler, G. Bona, F. Horst, I. Massarek, and B. Offrein, Silicon oxynitride layers for optical waveguide applications. Journal of the Electrochemical Society, 2000. 147(6): p. 2237-2241.

9. Peters, D., K. Fischer, and J. Müller, Integrated optics based on silicon oxynitride thin films deposited on silicon substrates for sensor applications. Sensors and Actuators A: Physical, 1991. 26(1): p. 425431.

10. Akca, B.I., Spectral-domain optical coherence tomography on a silicon chip. 2012.

11. Ismail, N., Integrated raman spectrometers for applications in health and medicine. 2012: Enschede. p. 141.

12. Wörhoff, K., P.V. Lambek, and A. Driessen, Design, tolerance analysis, and fabrication of silicon oxynitride based planar optical waveguides for communication devices. Journal of Lightwave Technology, 1999. 17(8): p. 1401-1407.

13. Roeloffzen, C.G.H., R.M.d. Ridder, and A. Driessen, Low-loss adiabatic bend using minimised chip area, in Proceedings 2000 IEEE/LEOS Symposium Benelux Chapter, X.J.M. Leijtens and J.H. Besten, Editors. 2000, Delft University of Technology: Delft. p. 175-178.

14. Kapron, F.P., D.B. Keck, and R.D. Maurer, Radiation Losses in Glass Optical Waveguides. Applied Physics Letters, 1970. 17(10): p. 423-+.

15. Roeloffzen, C.G.H., Passband Flattened Binary-tree Structured Add-drop Multiplexers Using SiON Waveguide Technology. 2002.

16. Wang, P., G. Brambilla, Y. Semenova, Q. Wu, and G. Farrell, Design of an extra-low-loss broadband Y-branch waveguide splitter based on a tapered MMI structure. 2011.

17. Wang, Q., J. Lu, and S. He, Optimal design method of a low-loss broadband Y branch with a multimode waveguide section. Applied Optics, 2002. 41(36): p. 7644-7649.

18. Sasaki, H. and N. Mikoshiba, Normalised power transmission in single mode optical branching waveguides. Electronics Letters, 1981. 17(3): p. 136-138. 
19. Huang, W.-P. and C. Xu, Simulation of three-dimensional optical waveguides by a full-vector beam propagation method. Quantum Electronics, IEEE Journal of, 1993. 29(10): p. 2639-2649.

20. Kunz, K.S. and R.J. Luebbers, The finite difference time domain method for electromagnetics. 1993: CRC press. 


\section{Chapter 4}

\section{Integrated micro-ball lens technology}

\subsection{Introduction}

Light coupling between an integrated optical chip and free space is an important aspect in many applications. Typical waveguide systems have a mode size on the order of $1-3 \mu \mathrm{m}$, acting as very tight apertures at the end facets of the waveguide where the light is coupled out of the chip. These small apertures lead to strong divergence, on the order of tens of degrees, of the out-coupled beam. However, many applications require a collimated or focused light beam. Examples are on-chip optical coherence tomography (OCT) [1, 2] and Raman spectroscopy [3]. A widely exploited approach is the use of one or more objective lenses to collimate or focus the light that emerges from the waveguide. However, the use of external lenses leads to a bulky setup requiring careful alignment, which would void the key advantages of using integrated optical chips. Therefore, an on-chip solution is preferred. Wei et al. demonstrated the use of a planar elliptic coupler at the end of a waveguide to focus the exit light in the horizontal direction [4]. Ismail et al. modified an arrayed waveguide grating for light delivery to, and light collection from, a focal point in the horizontal direction [5]. Gmachl et al. demonstrated a reduction in vertical divergence using the surface-plasmon effect by fabricating a vertical gold grating at the end facet of a quantum-cascade laser cavity [6]. A drawback of all these methods is that they only reduce the divergence in one of the two lateral directions.

A major improvement would be an integrated solution to shape the exiting beam in both horizontal and vertical directions. A tapered waveguide or inverse-tapered coupler can enlarge the mode diameter to reduce the divergence angle in both directions [7]. However, it is technologically challenging to fabricate a vertical taper or a sharp inverse taper. Linear tapers do not allow focusing the light, and the fabrication of nonlinear vertical tapers poses additional difficulties. Focusing grating couplers can couple the light out of the wafer plane,

Parts of this chapter have been published as: Chang, L., M. Dijkstra, N. Ismail, M. Pollnau, R.M. de Ridder, K. Wörhoff, V. Subramaniam, and J.S. Kanger, Waveguide-coupled micro-ball lens array suitable for mass fabrication. Optics Express, 2015. 23(17): p. 22414-22423. 
which is very useful for wafer-scale testing [8]. However, gratings have inherent spectral limitations, especially in a low-refractive-index-contrast waveguide technology, making them less suitable for broadband applications. For example, a wavelength-insensitive solution will be preferred for applications like OCT, which require a broad wavelength range to achieve excellent in-depth resolution.

In this chapter, we demonstrate a method that accomplishes two-dimensional, wavelength-tolerant collimation or focusing of light coupled out of high-numerical-aperture channel waveguides by integrating micrometer-sized ball lenses with the optical waveguide chip. In the field of optical communication, commercially available ball lenses are used to realize efficient fiber-to-fiber or waveguide-to-fiber coupling [9-11]. In these studies, the ball lenses need to be aligned one by one, which is a precise and time consuming task. There are several studies on the fabrication of on-chip micro-ball lens arrays, using a variety of approaches, which could reduce the alignment issue $[12,13]$. Our approach allows the integration of ball-lens fabrication with the fabrication of the waveguide structures, thus making the procedure suitable for mass production. Precise photolithographic alignment enables accurate positioning of all micro-ball lenses with respect to the optical waveguides.

We describe a process for fabricating polymeric micro-ball lenses that are aligned to silicon oxynitride $(\mathrm{SiON})$ waveguides with silicon dioxide $\left(\mathrm{SiO}_{2}\right)$ cladding layers, on a silicon ( $\mathrm{Si}$ ) substrate. Design, fabrication, and characterization of a microlens array for application in an on-chip OCT system are discussed.

\subsection{Micro-ball lens design}

Figure 4.2.1(a) shows a schematic of the micro-ball lens integrated onto a SiON channel waveguide chip. The basic idea is to form a micro-ball lens by reflowing a photoresist cylinder. In this design, two masks (corresponding to two major fabrication steps) are needed to integrate the micro-ball lenses with a given waveguide chip. The first major step is etching a platform through the $\mathrm{SiO}_{2}$ cladding layer and into the $\mathrm{Si}$ substrate, in front of the waveguides. This lowered platform allows the vertical alignment of the micro-ball lens center to the waveguide facet. After depositing a photoresist layer of appropriate thickness, the second major step is patterning cylinders into the photoresist, which, after thermal treatment, will result in a reflowed spherically shaped microlens structure. The details of each fabrication step are described in the fabrication process section. 
(a)

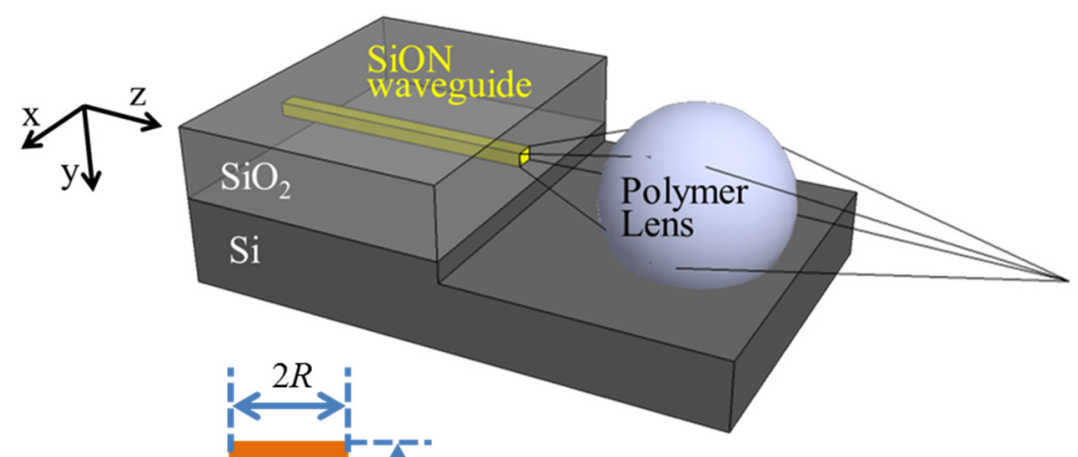

(b)

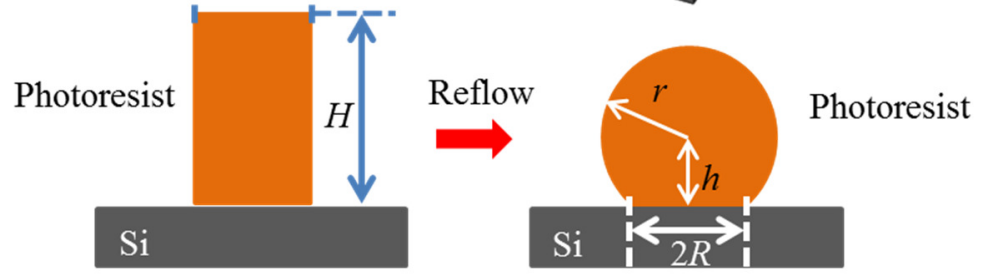

(c)

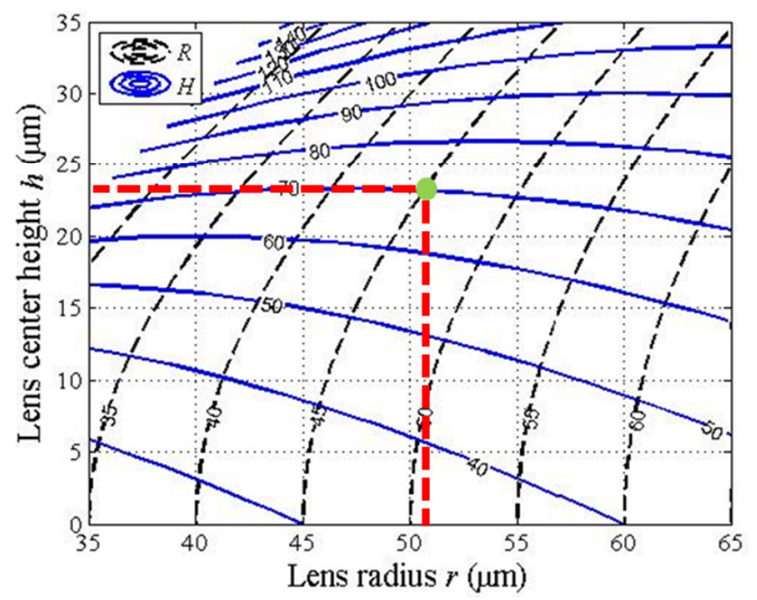

Fig. 4.2.1. (a) Schematic layout of a micro-ball lens integrated onto a SiON channel waveguide chip. (b) Schematic cross-section of the photoresist cylinder and its reflowed structure. (c) Diagram allows determining cylinder radius $R$ (thin black dashed curves) and height $H$ (thin blue solid curves) for a desired lens radius $r$ and center height $h$. The thick dashed red lines show that a lens with $r=51 \mu \mathrm{m}$, $h=23 \mu \mathrm{m}$ is obtained from a cylinder with $R \approx 45 \mu \mathrm{m}, H \approx 70 \mu \mathrm{m}$.

Fig. 4.2.1(b) shows the geometrical dimension of a photoresist cylinder before the reflow and the micro-ball lens after the reflow. The radius $r$ and center height $h$ of the microball lens are determined by the thickness $H$ of the photoresist layer and the radius $R$ of the patterned cylinder. The geometric model of the micro-ball lens is based on two assumptions. Firstly, the volume of the photoresist does not change during reflow. Secondly, the contact area of the Si substrate and the photoresist remains constant, due to the pinning effect provided by hexamethyldisilazane (HMDS) adhesion layer between the photoresist and Si. 
(This pinning effect is discussed in Section 4.3.4.) Given these assumptions, the radius $r$ and center height $h$ of the lens can be calculated as a function of the initial cylinder height $H$ and radius $R$.

The volume $V$ of the photoresist in its form of a cylinder before reflow is given by:

$$
V=V_{\text {cylinder }}=\pi R^{2} H .
$$

The same volume $V$ of the photoresist in the form of a micro-ball lens after reflow can be calculated as:

$$
V=V_{\text {ball-lens }}=\frac{2}{3} \pi r^{3}+\pi r^{2} h-\frac{1}{3} \pi h^{3} .
$$

Because the radius $R$ of the contact area is assumed to be constant, the following relation holds:

$$
r^{2}=h^{2}+R^{2}
$$

The effective focal length $f$ (distance between the ball center to the focal point) of a ball lens in air can be calculated with:

$$
f=\frac{n}{2(n-1)} r,
$$

where $n$ is the refractive index of the photoresist, which in our case is equal to 1.59 at a wavelength of $\lambda=1550 \mathrm{~nm}$.

Equations (4.2.1)-(4.2.3) are used to calculate the relationship between the cylinder height $H$, the cylinder radius $R$, the lens center height $h$, and the lens radius $r$. The calculated results are shown in Fig. 4.2.1(c). This graph allows one to determine the required dimensions of the cylinder $(R, H)$ to achieve the target dimensions of the lens $(r, h)$.

In order to fabricate appropriate lenses for a specific application, a set of design parameters can be obtained from the calculations above. The focal length $f$ of the micro-ball lens should be determined by the mode shape of the waveguide and the desired beam shape after the lens. In our example, an $f$ has been chosen around $69 \mu \mathrm{m}$ to collimate the light from our waveguide to a beam close to $40 \mu \mathrm{m}$ in diameter. From Equation (4.2.4), the required lens radius $r$ can be calculated as $r=51 \mu \mathrm{m}$. The clear aperture of the lens should be larger than the beam diameter in the lens to ensure a low distortion of the beam. The clear aperture of our micro-ball lens is limited by the lens center height $h$. In this example, $h$ should be larger than half the beam diameter, which is $20 \mu \mathrm{m}$. Considering a Gaussian beam, there is energy outside of the $1 / e^{2}$ beam radius and it is extended to infinity, thus, a larger $h$ provides a better result. Taking $h=23 \mu \mathrm{m}$ and $r=51 \mu \mathrm{m}$ as an example, Fig. 4.2.1(c) shows a required cylinder radius $R \approx 45 \mu \mathrm{m}$ and height $H \approx 70 \mu \mathrm{m}$ to produce the desired micro-ball lens.

Given a lens radius $r$, the center height $h$ of the lens can, in principle, be chosen freely 
between a minimum value set by the aperture $(20 \mu \mathrm{m})$ of the emerging beam at the position of the lens and a maximum value set by the lens radius $r(51 \mu \mathrm{m})$. However, for increasing values of $h$, larger values for the cylinder height $H$ are required. Given the fact that the fabrication process becomes increasingly difficult for larger photoresist layer thicknesses $H$ [14], the best choice for $h$ would be close to the minimum value.

Depending on the application, the distance between the facet of the waveguide and the lens center can be arranged freely to achieve either a diverged, collimated, or focused beam behind the micro-ball lens. There are, however, limitations. On the one hand, because the lens should not touch the waveguide facet to avoid deformation of the shape of the lens during the reflow, it should not be placed too close to the waveguide facet. On the other hand, because it should be avoided that the diverging beam coming out from the waveguide hits the substrate and creates a mirror reflection from the substrate, the lens should not be placed too far from the waveguide facet.

\subsection{Fabrication process}

The fabrication of the micro-ball lenses on a SiON waveguide wafer involves many steps. In order to have a clear overview of this technology, all the key fabrication steps are summarized in Section 4.3.1. The details of a few challenging fabrication steps are discussed in Sections 4.3.2 to 4.3.4. These details are intended to provide a better understanding of the fabrication process thus reducing the fabrication parameter optimization time in case of new lens designs with different lens sizes in the future. Section 4.3.5 summarizes a few typical failures during the fabrication. Possible reason of these failures are discussed which can be used in further optimization the fabrication process and improve the yield. A detailed step by step process including all process parameters are listed in the appendix (A.3).

\subsubsection{Major fabrication steps}

The process flow charts for etching the platform and fabricating the lens are shown in Fig. 4.3.1 and Fig. 4.3.2 respectively.

The starting point is a $100 \mathrm{~mm}$ diameter $<100>$ silicon wafer with SiON channel waveguides as shown in Fig. 4.3.1(a). The fabrication of the SiON channel waveguide is described in Section 3.2.

The fabrication process shown in Fig.4.3.1 consists four major parts. First, on top of the top cladding, a $1.1 \mu \mathrm{m}$ thick LPCVD poly-silicon layer is grown [dark grey layer in Fig. 4.3.1(b)]. This layer is applied as a hard mask during the deep $\mathrm{SiO}_{2}$ etching. 


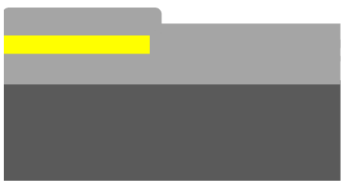

(a)

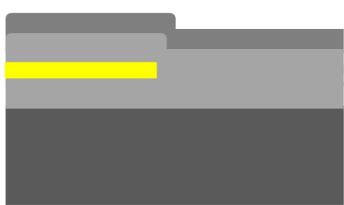

(b)

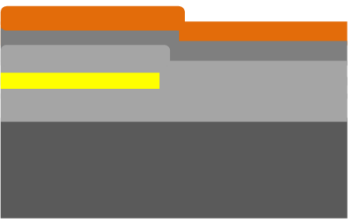

(c)

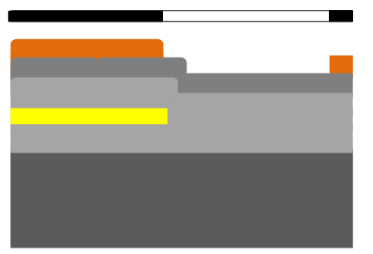

(d)

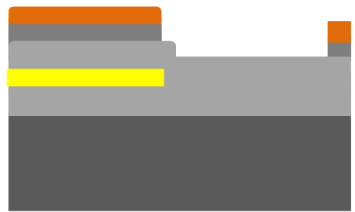

(e)

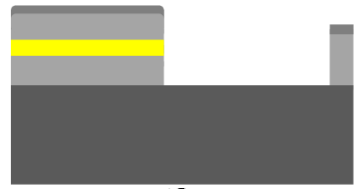

(f) $\leftarrow$ Platform Etch Mask

Photoresist

Poly-silicon

$\mathrm{SiO}_{2}$

PECVD SiON

$\mathrm{Si}$

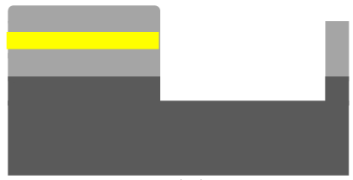

(g)

Fig. 4.3.1. Process flow chart for etching the platform on which the lenses are to be fabricated. The different materials are color-coded. For an explanation of the different steps see the main text.

Second, in order to pattern the poly-silicon mask, a $1.7 \mu \mathrm{m}$ thick positive photoresist (Olin OiR 907-17) layer is spin coated (4000 rpm, $30 \mathrm{~s}$ ) on top of the poly-silicon layer [orange layer in Fig. 4.3.1(c)] and then patterned with the platform etch mask. The resulting cross section is schematically shown in Fig. 4.3.1(d).

Third, the poly-silicon layer is dry-etched for 50 seconds in a Tetske machine by an $\mathrm{SF}_{6}$ based reactive ion etch (RIE) process. The schematic etch result is shown in Fig. 4.3.1(e). The etch rate of the applied RIE recipe strongly depends on the etch 'load', i.e., the required etching time has a negative correlation to the area covered with photoresist.

Fourth, the $12 \mu \mathrm{m}$ thick $\mathrm{SiO}_{2}$ stack is etched for $27 \mathrm{~min}$. in an Adixen AMS $100 \mathrm{DE}$ RIE machine, and all of the photoresist 907-17 layer and most of the poly-silicon layer is etched away as shown in Fig. 4.3.1(f). Thereafter the silicon is etched by a Bosch high aspect ratio silicon (B-HARS) etch recipe in an Adixen AMS 100 SE RIE machine. In this step the remaining poly-silicon is removed and the platform pattern is etched into the silicon wafer to a depth of $16 \mu \mathrm{m}$ [as indicated in Fig. 4.3.1(g)]. The load dependence of this recipe results in an etching procedure which is separated into two stages. The first stage is a slow etching stage. The remaining poly-silicon layer together with the exposed Si substrate in the platform results in a $100 \%$ load, which leads to a very slow etch rate $(<1 \mu \mathrm{m} / \mathrm{min})$. The second stage starts as soon as the poly-silicon layer has been etched away. For our design the etch load is 
reduced to $1 \%$. Consequently the etch rate of the silicon substrate increases to $4 \mu \mathrm{m} / \mathrm{min}$. The height difference between the center of the waveguide and the bottom of the platform is $24 \mu \mathrm{m}$, which corresponds approximately to the desired center height of the micro-ball lens. Currently, this etch depth is controlled by stopping the etch process and measuring the etched depth. If the measured depth is not enough, etch continues until the depth value is less than $2 \mu \mathrm{m}$ different from the desired value. An accurate time control is possible in case of repeated mass production.

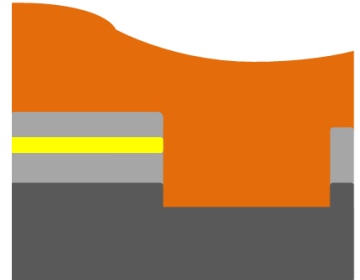

(a)

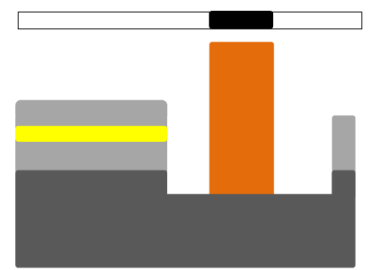

(b)

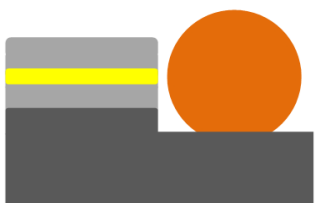

(c)

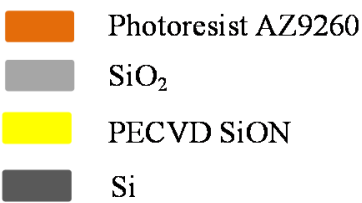

$\mathrm{Si}$ $\leftarrow \begin{aligned} & \text { Micro-ball lens } \\ & \text { Mask }\end{aligned}$

Fig. 4.3.2. Process flow chart for the fabrication of a photo-definable micro-ball lens array aligned to the channel waveguides.

The process flow chart of the photoresist micro-ball lens fabrication is shown in Fig. 4.3.2. The selected photoresist should fulfill the following requirements: reflowable to form a ball shape; low losses in the wavelength range of interest; suitable for the fabrication of a thick layer (in the order of $70 \mu \mathrm{m}$ thickness in our case). AZ9260 is one of the photoresists that fulfills all three requirements. For adhesion improvement vapor phase HMDS treatment was applied to the wafers prior to resist coating. By applying two AZ9260 coatings and several baking steps, the thick photoresist layer as shown in Fig. 4.3.2(a) is produced. This double layer coating procedure resulted in a photoresist thickness of $72 \pm 2 \mu \mathrm{m}$ inside the platform as measured with Dektak 8. The $2 \mu \mathrm{m}$ uncertainty is mainly caused by the photoresist layer inhomogeneities within one wafer.

For the patterning of the photoresist layer a photolithographic process is needed in which water molecules play an essential role during the exposure [15]. The AZ9260 is a diazonaphthoquinone-based resist, and its primary ketene photoproduct can react rapidly with water molecules to form a dissolution promoter, which will make the exposed resist soluble in the developer. However, if no water molecules are present, a slower reaction 
between the ketene photoproduct and the Novolak matrix [15] becomes dominant. This slower reaction produces an inhibitor which causes the exposed resist to become insoluble in the developer. During the baking steps water has been effectively removed from the resist layer. Therefore, rehydration in a humid environment is needed prior to exposure. Since the rehydration process relies on diffusion of the water molecules, the rehydration time is proportional to the square of the resist layer thickness. For a $70 \mu \mathrm{m}$ thick layer, a typical rehydration time in a $100 \%$ relative humidity environment $\left(20^{\circ} \mathrm{C}\right)$ is about 20 hours. A faster alternative way to rehydrate the resist layer is water immersion, which has been demonstrated by Lehar et al. [15]. It was found that sufficient rehydration in our resist layer is obtained by immersing the wafer into $30{ }^{\circ} \mathrm{C}$ water for $15 \mathrm{~min}$., followed by 3.5 hours in a cleanroom environment $\left(20^{\circ} \mathrm{C}, 48 \%\right.$ relative humidity). This reduction in process time can be attractive for mass production. The rehydrated resist film is exposed in an EV620 Mask Aligner at a dose of $3 \mathrm{~J} / \mathrm{cm}^{2}$. Subsequently, the resist is developed in the developer OPD4262 for 40 minutes. The schematic result after developing is shown in Fig. 4.3.2(b). The reflow of AZ9260 is performed on a $120^{\circ} \mathrm{C}$ hot plate for 2 minutes. Finally, the wafer is diced in order to bring the lens array close to the chip edge, as shown in Fig. 4.3.2(c).

\subsubsection{Details of AZ9260 double layer coating}

Different lens applications may require different thickness of the AZ9260 layer. The typical spin coating thickness of AZ9260 resist layer is about $6 \mu \mathrm{m}$ with a spin speed of $3000 \mathrm{rpm}$ for $30 \mathrm{~s}$. In our micro-ball lens application, a thickness around $70 \mu \mathrm{m}$ is needed, which is far from the typical thickness. In this section, we investigate the parameters that influence the resist layer thickness and present a way of making a $70 \mu \mathrm{m}$ thick double layer coating on a flat and non-flat surface.

During the spin coating, a few milliliters of photoresist is distributed over the substrate by the centrifugal force of the spinning substrate. The spin speed and spin time are the two main factors that determine the final thickness of the coated layer for a given photoresist. The layer thickness decreases with increasing spin speed. At the beginning of the spin process the layer thickness quickly decreases and remains almost constant after approximately tens of seconds. Thus, generally there are two ways to attain a layer of several tens of micrometers thick by reducing the spin speed or reducing the spin time.

Too low spin speeds (a few hundred rpm), however, cause a pronounced edge bead (Edge beads prevent sharp and well defined photolithography structures by keeping a gap between the mask and the resist surface during exposure.) and a non-reproducible resist film thickness as a consequence. Therefore, a much better way to produce a thick resist film is a high spin speed of approximately a few thousands rpm in combination with a high acceleration of approx. $1000 \mathrm{rpm} / \mathrm{s}$ for a short time (few seconds). 
For our application, the required layer thickness $(70 \mu \mathrm{m})$ is so large that a spin time as short as 1 second is still not enough as shown in Table 4.3.1. In order to avoid the problems mentioned above with very low spin speed, multiple coating steps are needed to further increase the layer thickness.

The thick photoresist film still has a relative high solvent concentration after spin coating. The application of a second layer could result in softening of, or complete dissolving of, the first layer, possibly resulting in strong inhomogeneities in the film thickness. Therefore, a proper drying (solvent evaporation) of the coated layer before the applying of the next layer is important to reduce the inhomogeneities of a multi-layer film. Even with proper drying, the wafer should be spun immediately (within a few seconds) after applying the resist onto the existing layer.

Fast drying of a thick (few tens of micrometers) resist film results in cracks in the layer. In order to gradually dry the resist film, the following steps were taken after spin coating:

1. $10 \sim 20 \mathrm{~min}$ room temperature (approximate $0.5 \mathrm{~min} / \mu \mathrm{m}$ );

2. $60{ }^{\circ} \mathrm{C}$ hot plate $5 \mathrm{~min}$;

3. $100{ }^{\circ} \mathrm{C}$ hot plate for a certain time (the time is depend on the following operation, such as few minutes for next layer or few tens minutes for photolithography);

4. Gradually (> $10 \mathrm{~min}$ ) cool down to the room temperature $\left(\mathrm{a} 60^{\circ} \mathrm{C}\right.$ hot plate $1 \mathrm{~min}$ is handy to slow down the cooling process to prevent cracks). During the steps mentioned above, pre-heating and pre-cooling is needed before and after in contact with each hot plate to prevent cracks in the resist film due to sudden temperature change. In practice, the pre-heating and pre-cooling is done by keeping the wafer approximately $1 \mathrm{~mm}$ above the hot plate with a tweezer for $20 \mathrm{~s}$.

In order to find the proper spin coating parameters for achieving the desired film thickness, several spin coating tests were done using the drying step mentioned above. The time on a $100{ }^{\circ} \mathrm{C}$ hot plate was 3 mins in these tests and the results are listed in Table 4.3.1. The 900-1500-900 and $1.3 \mathrm{~s}$ in the second row first column, as an example, describes the spin profile. In this example, the spin is accelerated with $900 \mathrm{rpm} / \mathrm{s}$ to $1500 \mathrm{rpm}$ ( $1.7 \mathrm{~s}$ acceleration time) and keep at $1500 \mathrm{rpm}$ for 1.3 second and then decelerate with $900 \mathrm{rpm} / \mathrm{s}$ to full stop. The thickness of the second layer is obtained by measuring the thickness of the double layer and subtracting the thickness of the first layer. The thickness of the third layer is obtained in a similar way as was done for the second layer. The uncertainty in the measured thickness in Table 4.3.1 reflects the thickness inhomogeneities within one wafer. 
Table 4.3.1. AZ9260 spin coating layer thickness obtained for different spin profiles.

\begin{tabular}{|c|c|c|c|}
\hline Spin profile & $\begin{array}{c}\text { Thickness of the } \\
\text { first layer }(\mu \mathrm{m})\end{array}$ & $\begin{array}{c}\text { Thickness of the } \\
\text { second layer }(\mu \mathrm{m})\end{array}$ & $\begin{array}{c}\text { Thickness of the } \\
\text { third layer }(\mu \mathrm{m})\end{array}$ \\
\hline $\begin{array}{c}900-1500-900 \\
1.3 \mathrm{~s}\end{array}$ & $38.5 \pm 0.5$ & $42.5 \pm 2$ & 1 \\
\hline $\begin{array}{c}1000-3000-1000 \\
1 \mathrm{~s}\end{array}$ & $15.7 \pm 0.3$ & $21.6 \pm 1.4$ & $18.9 \pm 3.5$ \\
\hline $1000-3000-1000$ & $12.9 \pm 0.2$ & $\backslash$ & $\backslash$ \\
\hline $1000-3000-1000$ & $11.5 \pm 0.2$ & $\backslash$ & \\
\hline $5 \mathrm{~s}$ & & & $\checkmark$ \\
\hline
\end{tabular}

In our lens design, the acceptable resist layer thickness uncertainty is $\pm 2 \mu \mathrm{m}$ for a $70 \mu \mathrm{m}$ thick layer. As shown in Table 4.3.1, a single layer coating is too thin to be used in our application. Table 4.3.1 shows that the layer thickness uncertainty increases with increasing layer thickness and increasing number of layers. The thickness uncertainty in case of a 3-layer coating is too large. Therefore, a double layer coating is optimal for our lens application.

In our design, the lens is located in an etched platform, where the resist film thickness is found to be different from the one on a flat surface. Several experiments were done (Fig. 4.3.3) in order to find out the optimal spin profile combination to achieve the target thickness in an etched platform.

The resist layer thickness reduction during the drying process has a direct effect on the dried layer thickness in a platform. The resist layer thickness before drying is not able to be measured directly in the MESA+ cleanroom. Thus a wafer with $30 \mu \mathrm{m}$ deep etched platforms is used [Fig. 4.3.3(a) and Fig. 4.3.3(b)] to measure this thickness reduction. Resist is poured onto the wafer and fills up the $30 \mu \mathrm{m}$ deep platforms. A blade is used to scrape the resist from the wafer except for the resist in the platforms. This leaves a $30 \pm 0.1 \mu \mathrm{m}$ thick resist layer before drying. The dried layer thickness is measured to be $12.9 \pm 0.1 \mu \mathrm{m}$, which is $43 \%$ of the undried layer as shown in Fig. 4.3.3(a). The resist pouring and scraping is repeated on the same wafer as shown in Fig. 4.3.3(b), to measure the thickness reduction in case of a double layer. The same $7.4 / 17.1=43 \%$ remaining thickness is measured. 


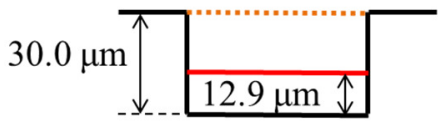

First fill

(a)

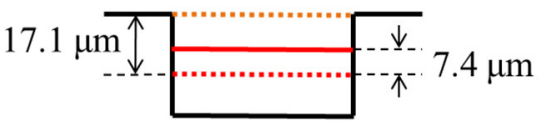

Second fill

(b)

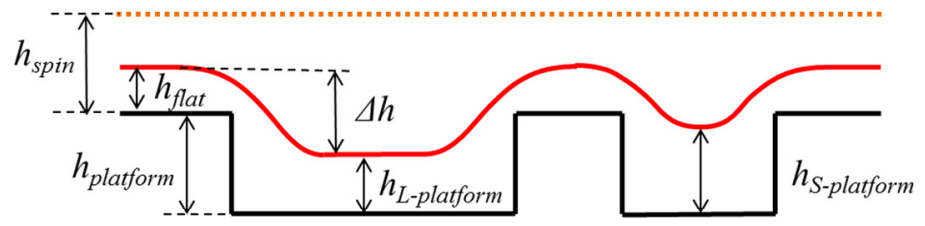

(c)

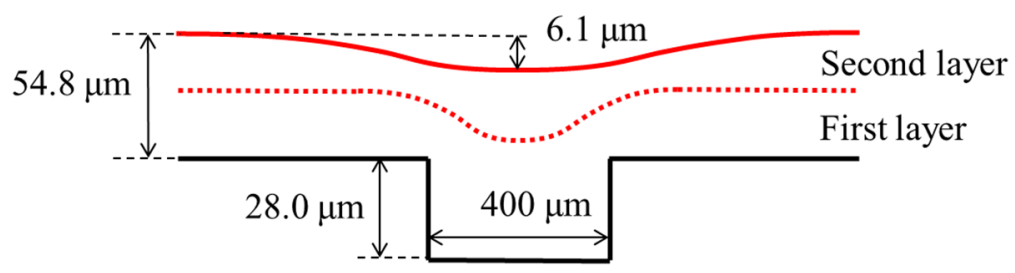

(d)

Fig. 4.3.3. Photoresist layer thickness and its morphology on non-flat surface. The meanings of the lines in these figures are: etched wafer surface (black solid line); resist level before drying (orange dotted line); resist level after drying (red solid line); and the first resist layer level after drying (red dotted line). (a) The experiment to measure the resist layer thickness reduction during drying by filling resist to a $30 \mu \mathrm{m}$ deep hold and drying. (b) The same experiment as in (a) is repeated twice to measure the thickness reduction in case of a double layer resist. (c) Schematic of the resist layer on a non-flat surface, with larger and small etched platform, after spin coating and drying. (d) Measured double layer resist thickness around a $400 \mu \mathrm{m}$ width by few millimeter long platform.

In case of a thick resist layer spin coated on the wafer, the resist layer thickness before drying is indicated in Fig. 4.3.3(c).The dried layer thickness on a flat part of the wafer is $h_{\text {flat }}=0.43 \cdot h_{\text {spin }}$. The thickness in a large platform (larger than approx. $0.6 \mathrm{~mm}$ in both edges for the resist layer thickness we used) is $h_{L-\text { plafform }}=0.43 \cdot\left(h_{\text {spin }}+h_{\text {plafform }}\right)$, where $h_{\text {plafform }}$ is the depth of the platform. The dried resist surface height difference is $\Delta h=0.57 \cdot h_{\text {plaform }}$ between a flat part and a larger platform, which is independent from the resist layer thickness. These relations can be used to estimate the resist thickness in a large etched platform based on the resist thickness data of a flat surface. 
The resist thickness in a small platform (our current design) is not easy to calculate as in case of a larger platform. During the drying process, the resist still has a certain mobility to flow into the platform due to the lowered surface. In a small platform, the flowed in resist from the side reaches the center of the platform which results in an increased resist thickness ( $h$ s-platform) in the platform as shown in Fig. 4.3.3(c). Therefore, the resist thickness in a small platform also depends on the size and shape of the platform. A systematic investigation with different platform sizes and shapes may results in a more quantitative relationship to the layer thickness in future work.

Experiments with the designed platform size ( $400 \mu \mathrm{m}$ wide and a few centimeters long) are shown in Fig. 4.3.3(d). The resist layer thickness outside the platform is known based on spin coating data on a flat surface wafer (Table 4.3.1). The double layer coating further reduces the surface height difference $\Delta h$ to about $6 \mu \mathrm{m}$ in case of a $70 \sim 80 \mu \mathrm{m}$ resist layer in the platform. Since $\Delta h$ is the only thickness related parameter can be measured before patterning and developing the resist layer. Knowledge of this height difference $\Delta h$ is important for estimating the resist thickness in the platform. The resist thickness in the platform is the parameter need to be controlled for proper lens fabrication. It is very useful to fine tune the spin coating parameters to achieve the desired thickness in the platform.

In this section, we summarized the fabrication challenges of thick AZ9260 layer on nonflat surfaces. We first discussed the ways of tuning resist layer thickness during spin coating. Then we motivated the use of double layer coating instead of single layer coating in case of a very thick layer. We then described how the double layer coating is realized. Finally, we discussed the effects of thick resist layer spin coated on a non-flat surface and how to achieve a certain thickness of the AZ9260 layer in the etched micro-lens platforms. This information is useful to reduce the parameters optimization time in case a new lens design with a different AZ9260 layer thickness is required.

\subsubsection{Details related to AZ9260 rehydration}

The rehydration of a thick AZ9260 layer was the most challenge part during the optimization of the micro-ball lens fabrication process. The importance of the rehydration has been introduced in Section 4.3.1. In this section, we discuss in detail about three parameters which influence the rehydration process, namely soft bake time, rehydration time and temperature.

The first influencing factor is the soft bake time at $100{ }^{\circ} \mathrm{C}$ after the second layer coating. Water is effectively removed from the resist layer during this bake. However, this bake is needed to remove the solvent from the resist layer to minimize the dark erosion (development of the unexposed resist) during development. Therefore, experiments on $80 \mu \mathrm{m}$ thick AZ9260 layers on flat wafers with different bake times are done to investigate the influence of the soft bake time. 
Except the soft bake time, all other rehydration relevant experimental parameters were kept the same as follows:

- Immersion in $20{ }^{\circ} \mathrm{C}$ deionized (DI) water for rehydration (20 mins).

- Spin dry the wafer with $2000 \mathrm{rpm}$ without $\mathrm{N}_{2}$ flow. (This is used to remove remaining water droplets on the wafer after the wafer is taken out from the water. This is a lower drying speed than what is standard in our cleanroom. The lower speed is preferred to reduce the resist layer cracking risk due to the temperature drop caused by water evaporation.)

- Time of the wafer exposed to the cleanroom air $\left(20{ }^{\circ} \mathrm{C}, 43 \%\right.$ relative humidity) between the spin drying and the exposure in the photolithography machine was about 50 min. During this time the water in the resist layer keeps diffusing deeper in the layer. The water in the air also diffuses into the layer.

Insufficient rehydration is achieved with the above mentioned parameters. This leads to an incomplete resist layer development even in case of sufficient ultraviolet (UV) exposure (see Section 4.3.1, $3 \mathrm{~J} / \mathrm{cm}^{2}$ dose with an EV620 Mask Aligner, UV intensity is $12 \mathrm{~mW} / \mathrm{cm}^{2}$ ) and sufficient long development time (in the order of few tens of minutes until the development stops). The resist layer development stops at a depth where the water concentration in the resist is too low. The developed film thickness (the film thickness removed during development) is measured for different soft bake times (Fig. 4.3.4). The observed developed film thickness is an indication of the water diffused depth.

The developed film thickness decreases with increasing soft bake time as shown in Fig. 4.3.4. On the other hand, the development results are better in case of a longer bake time as shown in the insets of Fig. 4.3.4. There are two resist cylinders in each of these insets. However, it is difficult in the inset (b) and not possible in the inset (a) to recognize the cylinder due to the bad development results. Exposure of a non-sufficiently baked resist layer creates $\mathrm{N}_{2}$ which leads to a well-known foaming effect due to the forming of $\mathrm{N}_{2}$ bubbles [16]. These bubbles are the defects in these inset images which are reducing as the increasing of the bake time. The large error bar in the developed film thickness measurement is a result of the non-flat surface caused by these bubbles.

The conclusion from the results in Fig. 4.3.4 is that the soft bake time at $100{ }^{\circ} \mathrm{C}$ needs $50 \mathrm{~min}$ at least for an $80 \mu \mathrm{m}$ AZ9260 layer to have an acceptable development result. The rehydration has to be increased in order to fully develop a resist layer with extended baked time. 


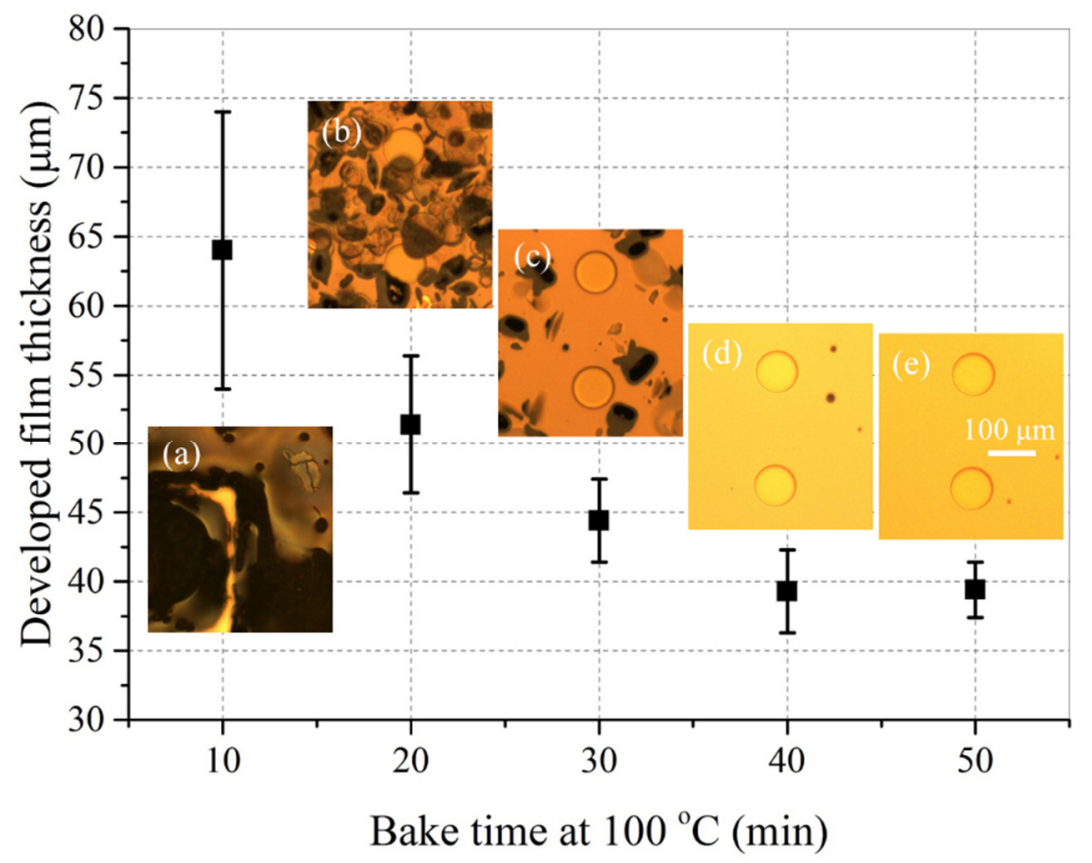

Fig. 4.3.4. Developed film thickness as function of the soft bake time at $100{ }^{\circ} \mathrm{C}$. The insets are microscopy images of the film after development. All the images are taken at the same area on the wafer with the same magnification. The scale bar is shown in inset (e). The color difference between pictures is due to the auto color balance of the microscope camera.

In the next few paragraphs, the influence on the rehydration by the water immersion time is discussed. The rehydration of the resist layer relies on water diffusion from the environment into the layer. Increasing the water concentration in the environment by immersion of the wafer in a water bath can effectively reduce the rehydration time (compared to rehydration in air), which has been demonstrated by Lehar et al. [15]. Experiments on $80 \mu \mathrm{m}$ thick AZ9260 layers on flat wafers with different water immersion time are done. The developed film thicknesses were measured and shown in Fig. 4.3.5. Except the water immersion time, all other relevant experimental parameters were kept the same:

- Soft bake time at $100{ }^{\circ} \mathrm{C}$ is $50 \mathrm{~min}$.

- DI water temperature is $20^{\circ} \mathrm{C}$ (room temperature).

- Spin dry the wafer with $2000 \mathrm{rpm}, 30 \mathrm{~s}$, without $\mathrm{N}_{2}$ flow.

- Time of the wafer exposed to the cleanroom air between the spin drying and the exposure was about $15 \mathrm{~min}$. (This time is kept as short as possible to minimize the water diffusion outside the water bath. In practice, \pm 5 min fluctuation is common due to manual preparation steps on the exposure machine.)

- Exposure dose is $1.8 \mathrm{~J} / \mathrm{cm}^{2}$. This dose is less than in previous experiments with 
different bake times. Nevertheless, it is still sufficient for the developed film thickness in these experiments. In case of non-sufficient dose, there will be an exposure limited (fixed) developed film thickness regardless the increasing water immersion time, which is not seen in our data (Fig. 4.3.5).

The developed film thickness increases with the water immersion time as shown in Fig. 4.3.5. All the developed results are in good shape and an example of a testing structure is shown in the inset (a) of Fig. 4.3.5. The error bars in Fig. 4.3.5 are much smaller than the one, also with 50 min bake time, in Fig. 4.3.4 due to the reduced presence of $\mathrm{N}_{2}$ bubbles as a result of lower exposer dose. In order to develop through a $70 \mu \mathrm{m}$ thick layer, an extended water immersion time is expected based on the plot in Fig. 4.3.5. However, an experiment with 50 min water immersion time shown a badly developed result as shown in the inset (b) in Fig. 4.3.5.

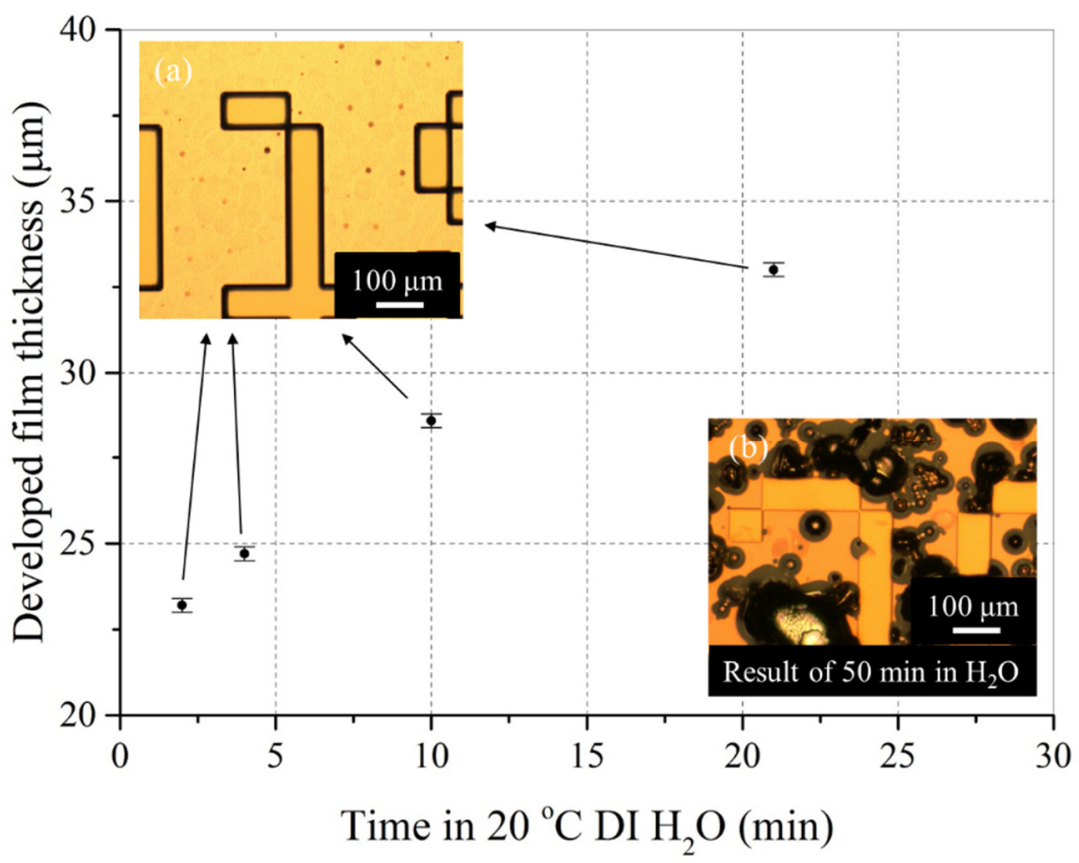

Fig. 4.3.5. Developed film thickness measured for different water immersion times at $20{ }^{\circ} \mathrm{C}$. Inset (a) is a microscope image of the film after development for an immersion time of $21 \mathrm{~min}$. At shorter water immersion times images of the film are very similar to the one in inset (a). Inset (b) shows a microscopy image of the film after development for an immersion time of $50 \mathrm{~min}$.

The conclusion from the results in Fig. 4.3.5 is that the developed film thickness increased with increasing water immersion time. However, a too long water immersion time (such as 50 minutes) has a negative influence on the quality of the remaining film.

Next, the influence on the rehydration by the water immersion temperature is discussed. 
Diffusion of water into the film is expected to be faster at higher temperatures. Experiments with different DI water temperatures, in which the wafer is immersed, are done and the results are shown in Table 4.3.2.

Except the water immersion temperature, all the other relevant experiment parameters were kept the same as follows:

- Soft bake time at $100{ }^{\circ} \mathrm{C}$ is $50 \mathrm{~min}$.

- Immerse time in DI water is $20 \mathrm{~min}$.

- Spin dry the wafer with $2000 \mathrm{rpm}, 30 \mathrm{~s}$, without $\mathrm{N}_{2}$ flow.

- Time of the wafer exposed to the cleanroom air between the spin drying and the exposure was about $20 \mathrm{~min}$.

- $\quad$ Exposure dose is $3 \mathrm{~J} / \mathrm{cm}^{2}$.

Table 4.3.2. Development results of an $80 \mu \mathrm{m}$ AZ9260 layer for different water immersion temperatures.

\begin{tabular}{|c|c|c|}
\hline $\mathrm{T}\left({ }^{\circ} \mathrm{C}\right)$ & $\begin{array}{c}\text { Developed film } \\
\text { thickness }(\mu \mathrm{m})\end{array}$ & \multicolumn{1}{|c|}{ Note } \\
\hline 20 & $30 \pm 3$ & All resist structures are in good shape. \\
\hline 30 & $47 \pm 3$ & $\begin{array}{l}\text { Some of the resist structures are missing. Most of the } \\
\text { remaining structures have certain damages. }\end{array}$ \\
\cline { 1 - 2 } 40 & $60 \pm 15$ & $\begin{array}{l}\text { Most resist structures are gone from the wafer. The } \\
\text { remaining structures are not usable due to their } \\
\text { incomplete shape. }\end{array}$ \\
\hline 50 & 1 & 1
\end{tabular}

The developed film thickness increases with increasing temperature (Table 4.3.2). However, for water temperatures of $40{ }^{\circ} \mathrm{C}$ and higher, the developed films are not useable due to damage. A side effect has also been noted during this experiment. If the rehydration temperature is too high, it reduces the adhesion force between the Si substrate and resist layer. Rehydration at $60{ }^{\circ} \mathrm{C}$ and $70{ }^{\circ} \mathrm{C}$ resulted in the removal of the entire layer.

Considering the experimental results above a two-step approach is used to rehydrate a $70 \mu \mathrm{m}$ thick resist layer. In the first step the wafer is kept in $30{ }^{\circ} \mathrm{C} \mathrm{DI}$ water for $15 \mathrm{~min}$, causing a relatively fast diffusion of water into the layer. The $15 \mathrm{~min}$ duration is short enough to prevent damage to the resist structures, however not long enough to fully rehydrate the whole layer. Therefore, in the second step the wafer is kept in contact with the cleanroom air $\left(20{ }^{\circ} \mathrm{C}\right.$ ) for approximately 3.5 hours before exposure. During this step water keeps diffusing deeper into the resist layer. Because of reduced temperature and water supply (less water 
concentration in air compare with the water bath) to the resist layer in this step, reduction of the adhesion between the resist and $\mathrm{Si}$ substrate is prevented. All the relevant experimental parameters for rehydration are summarized as follows:

- Soft bake time at $100{ }^{\circ} \mathrm{C}$ is $50 \mathrm{~min}$.

- Immerse time in $30{ }^{\circ} \mathrm{C}$ DI water is $15 \mathrm{~min}$.

- $\quad$ Spin dry the wafer with $2000 \mathrm{rpm}, 30 \mathrm{~s}$, without $\mathrm{N}_{2}$ flow.

- Time of the wafer exposed to the cleanroom air between the spin drying and the exposure was about 3.5 hours.

This two-step approach reduces the rehydration time (in a $20{ }^{\circ} \mathrm{C}$ and $100 \%$ relative humidity air environment) of a $70 \mu \mathrm{m}$ thick resist layer from more than 20 hours to less than 4 hours. This reduction in process time can be attractive for mass production.

In this section, we summarized the fabrication challenges of thick AZ9260 layers that have a proper rehydration for development. We first discussed the influence of the soft bake time on the rehydration process. Then we discuss the influence of the immersion time and water temperature of the water immersion step. Immersion of the wafer in a water bath improves the rehydration speed compared to rehydration in air only. However, immersing the wafer too long in water and/or using water with too high temperatures has a negative influence on the development result. Finally, a two-stage rehydration (short water immersion followed by rehydration in air) strategy is used in our lens fabrication. This strategy reduces the total rehydration time from more than 20 hours (rehydration in air only) to less than 4 hours.

\subsubsection{Pinning effect during the AZ9260 reflow}

During the resist reflow, the cylinder shaped resist is transformed into a spherical shaped ball lens. During this reflow the contact area of the resist with the Si-wafer remains constant. This pinning effect as mentioned earlier in the lens design section (Section 4.2) is one of the assumptions used to relate the shape of the ball lens to the shape of the cylinder and is the key to define the cylinder shape required for the designed ball lens properties. This section is to demonstrate this pinning effect experimentally.

Resist cylinders with the same radius and different heights are fabricated and reflowed as shown in Fig. 4.3.6. All images in Fig 4.3.6 are scanning electron microscope (SEM) images. The samples are coated with an ultrathin (few to few tens of nanometers in thickness) gold/palladium layer to improve the conductivity of the resist surface. This conducting coating is necessary to prevent charge accumulation on the non-conducting resist and enables high resolution image. 

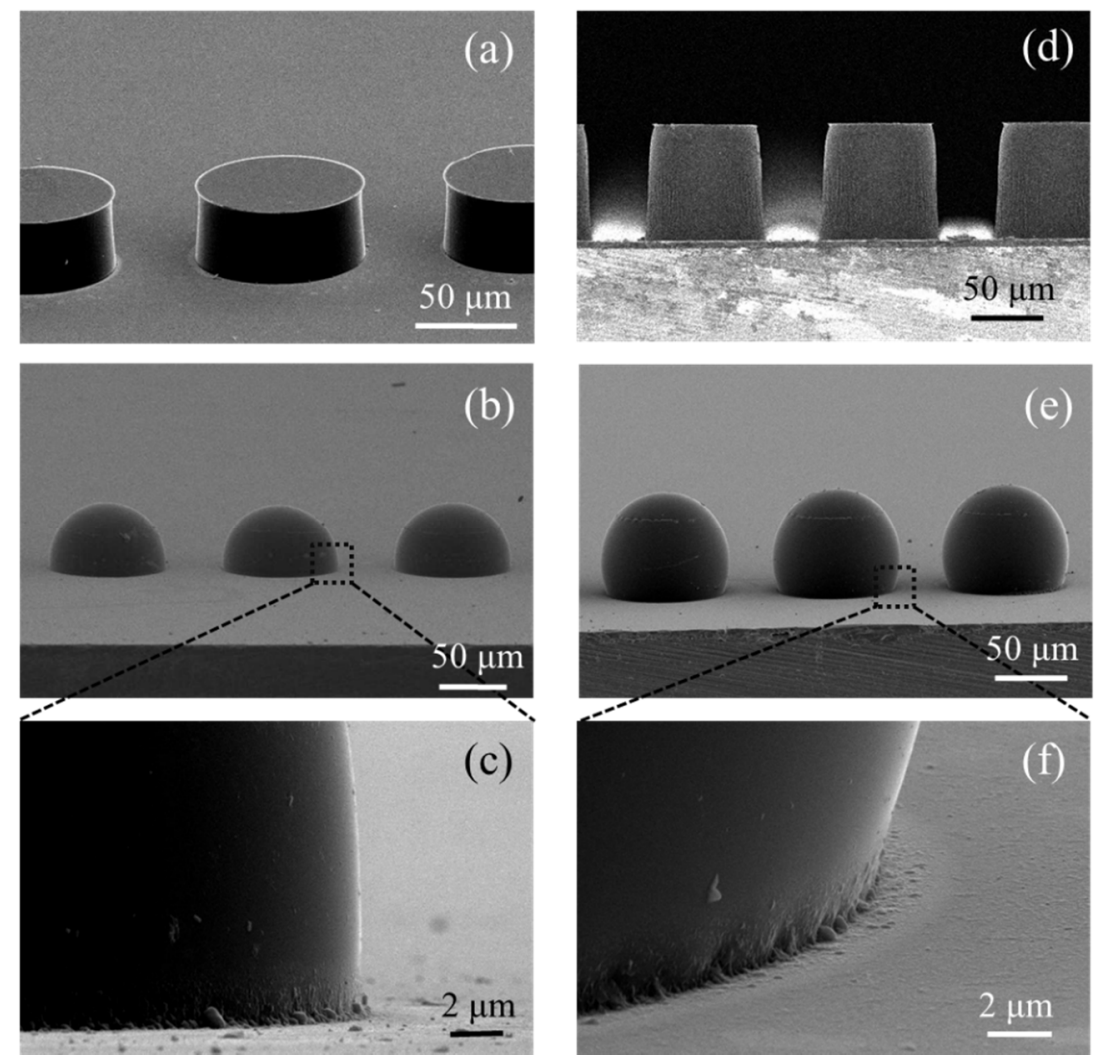

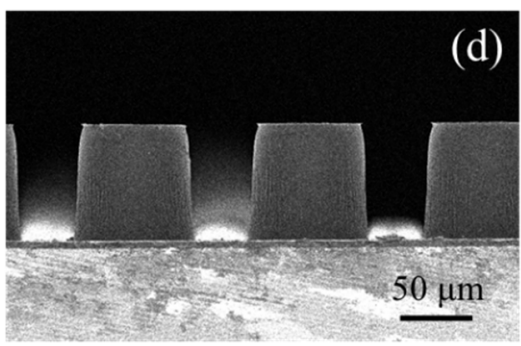

Fig. 4.3.6. SEM images of resist cylinders before and after reflow. (a) A cylinder with a radius of $45 \mu \mathrm{m}$ and a height of $38 \mu \mathrm{m}$ before reflow. (b) A cylinder of the same size of (a) after reflow. (c) Zoomed in image of the contact region between the resist and the Si substrate. (d) - (f) are the corresponding images as (a) - (c) for cylinders with a $45 \mu \mathrm{m}$ radius and a $80 \mu \mathrm{m}$ height.

The reflow condition was the same for both samples ( $2 \mathrm{~min}$ on a $120^{\circ} \mathrm{C}$ hot plate). The SEM images show that the radius of contact area $(45 \mu \mathrm{m})$ is not changed before and after the reflow. The zoomed in images, Fig. 4.3.6(c) and Fig. 4.3.6(f), show a clear difference in contact angle between the resist and the Si substrate. This is due to a combined effect of a pinned contact line on the Si surface and the surface tension of the melted resist.

The exact origin of the observed pinning effect is not fully understood at this moment. However, it very likely originates from the adhesion force between the resist and Si substrate. This adhesion force is provided by the HMDS layer that is coated on the Si surface. During the cleaning of the wafer after development of the resist layer, the HMDS is washed away by water at the location where no resist covering. Therefore, the remaining HMDS under the resist cylinders could be the element that provides the pinning effect. 


\subsubsection{Typical fabrication failures}

Two typical failures during the fabrication are summarized in this section. The possible reasons of these failures are discussed in this section, which can be useful for future optimization of the lens fabrication process and for improvement of the yield.

One of the typical failures is bubble forming during the resist reflow as shown in Fig. 4.3.7(a). Most of the lenses with bubbles are found in wafers which are reflowed shortly after the development and cleaning steps. It is very likely that large amounts of water diffused into the resist cylinder during the cleaning step (clean with DI water for $5 \sim 10 \mathrm{~min}$ ). The water quickly evaporates during reflow at $120^{\circ} \mathrm{C}$ and in this way forms bubbles. Due to the large viscosity of the resist and short reflow time $(2 \mathrm{~min})$, the bubble is not able to escape from the resist. On the other hand, if there is extended time before reflow (1 hour or more), the water concentration in the resist is reduced by exposing to air (cleanroom air with $43 \%$ relative humidity).

More systematic experiments are needed to better understand the reason of the bubble forming. With the current knowledge, we suggest to keep at least 1 hour waiting time before reflow of the resist cylinders to minimize bubble forming.

Another typical failure is lens peel off during wafer dicing as shown in Fig. 4.3.7(b). The dicing machine used is Loadpoint Micro Ace 3 dicing saw. Its dicing blade is cooled by a strong and constant water jet. Multiple times of dicing are needed to separate all the structures on a wafer. During the dicing, we found that after 4 to 5 times dicing, lenses start to disappear from the wafer. Two effects may contribute to this lens loss. First, water may get into the HMDS adhesion layer and weaken the adhesion force during the long (around 1 hour) time in which the lenses are in contact with the cooling water (see Section 4.3.3). Second, the strong water jet together with the small particles from the diced wafer could mechanically remove the lens.

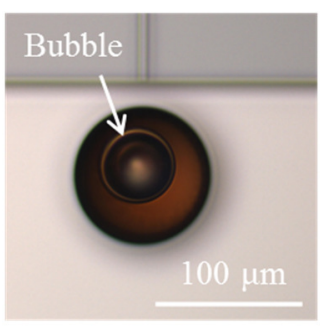

(a)
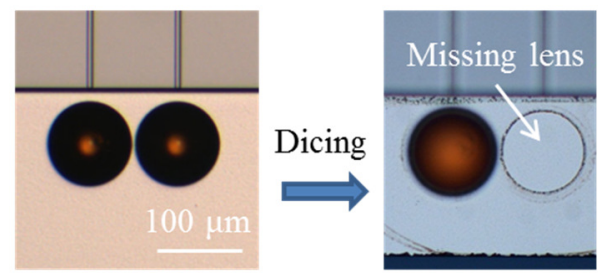

(b)

Fig. 4.3.7. Typical failures in the current lens fabrication. (a) Microscopy image of a lens with a bubble inside. (b) Microscopy images of two lenses before and after dicing. One lens is lost during dicing. 
In order to reduce the number of missing lenses in future fabrications, a reduced number of dicing steps is preferred. For example, by a better arrangement of the structures on the wafer. The finished dices can be taken out first to reduce its time exposed to the water jet. The speed of the dicing can be increased at positions without a waveguide facet to reduce the total dicing time and thus the water immersion time. An even better alternative is to dice the wafer with a dry dicing machine (not available in the MESA+ cleanroom at the moment), which will not have the problems mentioned above.

\subsection{Fabrication results}

Hundreds of micro-ball lenses were fabricated on three wafers. The yield (good shape and position by visual observation using a microscope) is above $95 \%$ before dicing and is reduced to $85 \%$ after dicing. Most common failures are lenses with bubbles or lenses missing as discussed in the Section 4.3.5.

Fabricated micro-ball lenses and waveguides are shown in Fig. 4.4.1. A bird's-eye view of a lens array before dicing is shown in Fig. 4.4.1(a). There is one micro-ball lens in front of each waveguide. The silicon surface of the platform is smooth enough to show clear mirror images of the lenses. A top view of the same micro-ball lens array after dicing is shown in Fig. 4.4.1(b). All the lenses are well aligned in front of each waveguide. The dicing edge is about $100 \mu \mathrm{m}$ from the lens edge. The lens radius $r=49 \pm 1 \mu \mathrm{m}$ is measured from the topview image. The lens center height $h=l /(2 \cos (\theta))=24 \pm 1 \mu \mathrm{m}$ is measured indirectly from the bird's-eye-view image by knowing the bitangent line segment length $l$ [as indicated in Fig. 4.4.1(c)] of the lens and its mirror image outline, as well as the angle $\theta$ of view of the image with respect to the wafer plane. The dimensions of all lenses are identical within the measurement accuracy and are in good agreement with the calculation based on Fig. 4.2.1(c).

The horizontal lens-waveguide alignment accuracy is limited by the lithographic process. In our case, the maximum inaccuracy is specified as $1 \mu \mathrm{m}$. Measurements on a few samples showed the actual misalignment error to be $0.7 \pm 0.2 \mu \mathrm{m}$. The vertical lens-waveguide alignment can be controlled with accuracy of $1 \mu \mathrm{m}$ for the ball lens size we used. The main contribution to the vertical alignment error is the cylinder height before reflow. We believe that the uncertainty introduced by our current manual double-coating procedure can be considerably reduced by using an automated photoresist spin coating machine.

To test the mechanical stability of the lenses, destructive experiments were performed with a Nordson Dage 4000Plus Bondtester. The measured average force to destroy/remove a micro-ball lens by pushing from the side is 6 centinewton $(\mathrm{cN})$ with a standard deviation of $1.5 \mathrm{cN}$. A micro-ball lens has a mass on the order of $10^{-6} \mathrm{~g}$, which means the lens should be able to withstand an acceleration in the order of $10^{7} \mathrm{~m} / \mathrm{s}^{2}$. This indicates a good mechanical 
stability of the lenses.
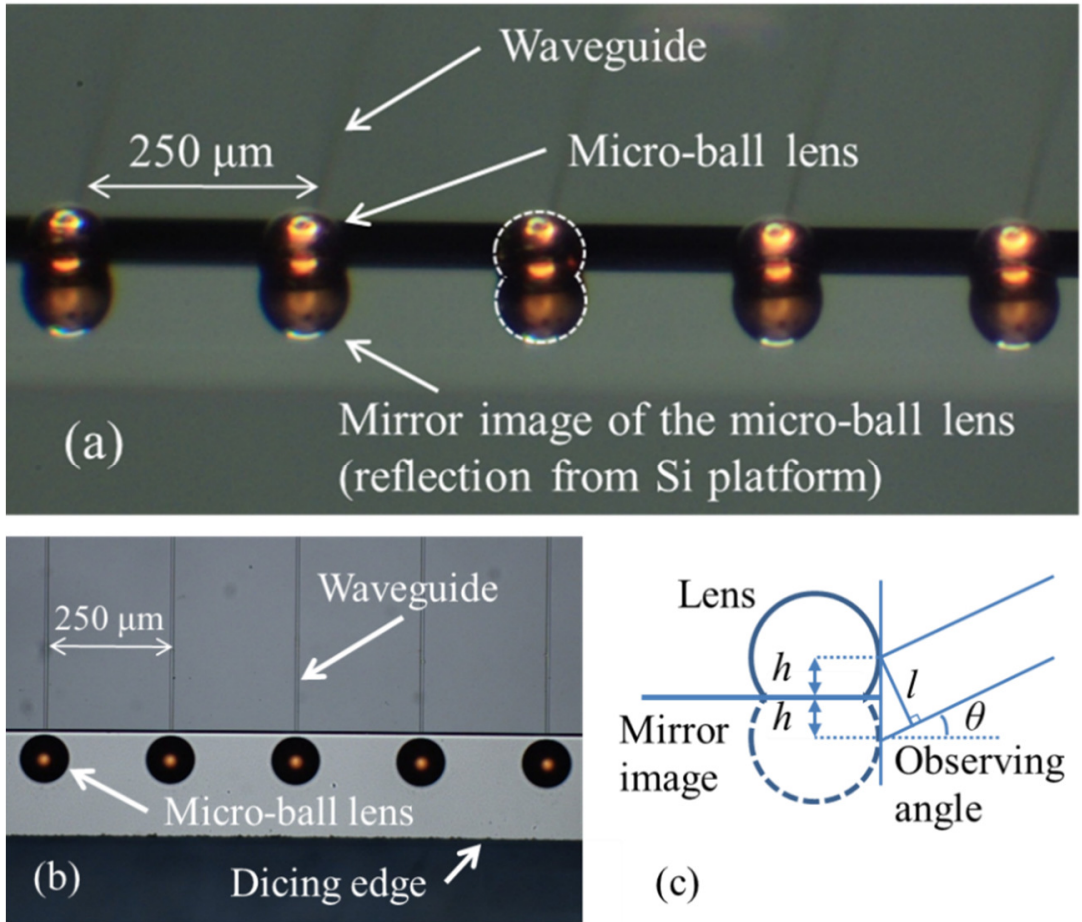

(c)

Fig. 4.4.1. (a) Bird's-eye view of a fabricated micro-ball lens array before dicing the chip. There are 5 waveguides at $250 \mu \mathrm{m}$ spacing in this image. The outline of the middle lens and its mirror image has been drawn to guide the eye. (b) Top view of a micro-ball lens array after dicing. (c) Geometry layout used to calculate the lens center height $h=l /(2 \cos (\theta))$ from the observed distance $l$ and observing angle $\theta$.

\subsection{Optical characterization and discussion}

In order to measure the optical performance of the micro-ball lenses, beam profiles behind the micro-ball lenses were measured with a home-built knife-edge setup at a wavelength of $1544 \mathrm{~nm}$. An example of a measurement is shown in Fig. 4.5.1. In this particular measurement, the knife edge is $1.2 \mathrm{~mm}$ away from the waveguide end. 

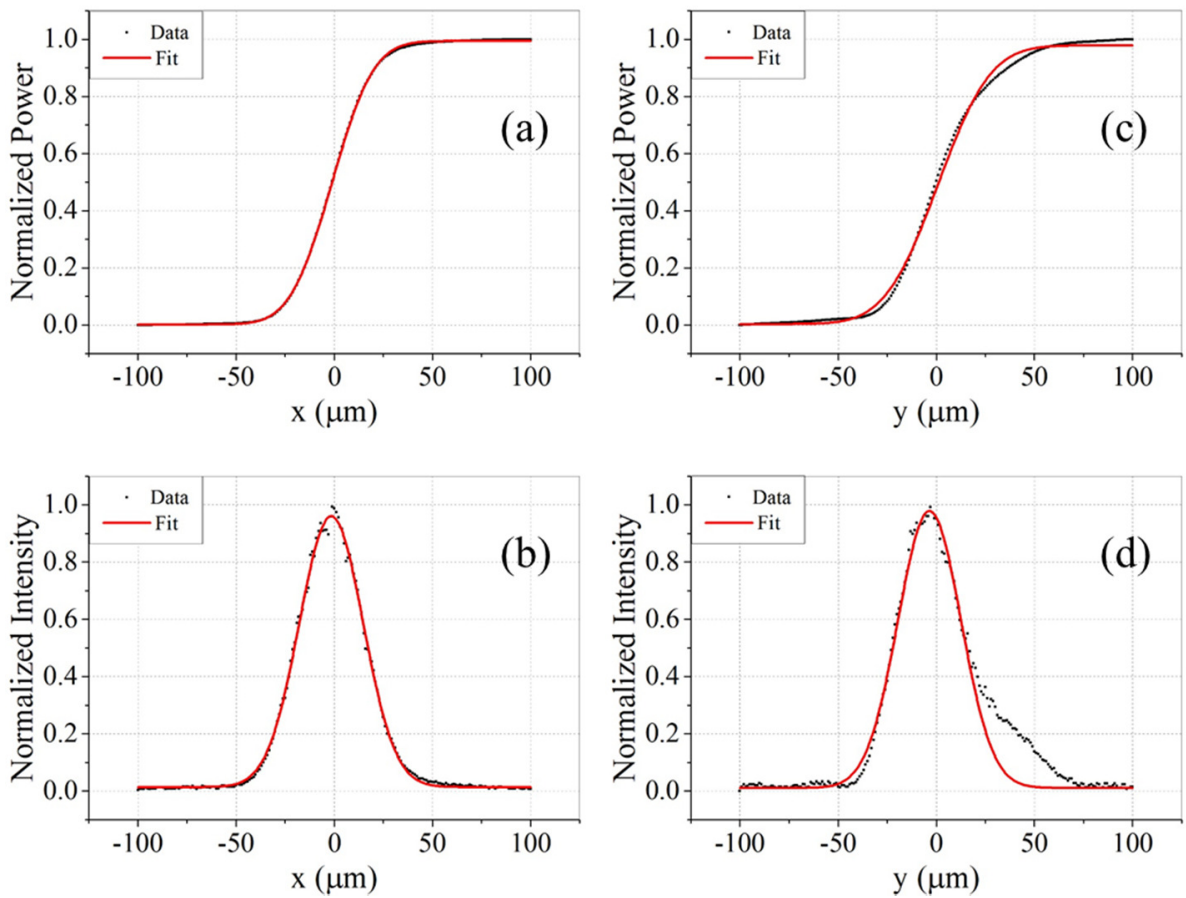

Fig. 4.5.1. Beam profile (at $\lambda=1544 \mathrm{~nm}$ ) behind a micro-ball lens measured with a knife-edge setup at $1.2 \mathrm{~mm}$ away from the waveguide end. (a) and (c) display the measured power and their fitting in the horizontal ( $x$ ) and vertical (y) direction, respectively. (b) and (d) display the derivative of the measured data and its Gaussian fitting of (a) and (c), respectively.

Fig. 4.5.1(a) shows the normalized power as measured during one knife-edge scan in the $x$ direction (parallel to the chip dicing edge). The data is fitted using the following expression [17] for the measured power $P$, which assumes a Gaussian intensity profile of the beam:

$$
P=P_{0}+\frac{P_{\max }}{2} \operatorname{erf}\left(\frac{\sqrt{2}\left(x-x_{0}\right)}{w}\right),
$$

with $P_{0}$, the background power, $P_{\max }$, the maximum power, $x_{0}$ the center position of the Gaussian intensity profile, and erf is a standard error function. The $1 / e^{2}$ beam radius $w$ is obtained by the fitting procedure. The result of this fit is shown in Fig. 4.5.1(a). The $x$ derivative of the measured power $P$ yields the intensity profile in the $x$ direction as shown in Fig. 4.5.1(b). In Figs. 4.5.1(c) and 4.5.1(d), the power and intensity profile in the $y$ direction (perpendicular to the chip plane) are shown. The positive $y$ direction in this case is defined from the lens center point down to the Si substrate. The values for the beam radius $w$ in $x$ and $y$ directions as obtained from the fit are $32.4 \mu \mathrm{m}$ and $32.1 \mu \mathrm{m}$, respectively. 
It can be observed from Figs. 4.5.1(b) and 4.5.1(d) that the beam shape in the $x$ direction fits much better to a Gaussian-shaped beam profile compared to the $y$ direction. In the $y$ direction, the lower part of the beam (at positive values for $y$ ) deviates from a Gaussian shape. This is due to the fact that the Gaussian beam has a tail extending to infinity and its lower part (outside the $\mathrm{e}^{-2}$ beam radius, where the intensity is not yet negligible) is disturbed by the bottom of the truncated lens. Diffraction of the disturbed Gaussian beam results in the observed extended tail in the $y$ direction.

For some applications, a better beam shape may be needed. In that case, the platform should be etched a few micrometers deeper and the micro-ball lens center should be elevated by the same amount to reduce blocking of the Gaussian tail by the platform.

To further analyze the optical performance of the lens, the beam radius $w(z)$ along the $x$ direction was measured at different positions $z$ along the optical axis for three different structures at wavelengths of $1544 \mathrm{~nm}$ and $1306 \mathrm{~nm}$ using the knife-edge method (Fig. 4.5.2). The data are fitted to the beam radius expected for a Gaussian beam as given by [17]:

$$
w(z)=w_{0} \sqrt{1+\left(\frac{z-z_{0}}{z_{R}}\right)^{2}} ; \text { with } z_{R}=\frac{\pi w_{0}^{2}}{\lambda},
$$

where $z_{0}$ is the position of the beam waist $w_{0}, \lambda$ is the wavelength, and $z_{R}$ is the Rayleigh range. Fitting the data using Equation 4.5.2 yields the size $w_{0}$ and location $z_{0}$ of the beam waist. The fit results for all three structures are good, confirming our earlier conclusion based on Fig. 4.5.1(b) that the beam profile along the $x$ direction is well approximated by a Gaussian distribution. The extended tail in the $y$ direction changes shape and position as a function of location $z$ which lead to a difficultly to accurately determine the beam radius $w(z)$ at all locations $z$. However, the main peak has similar beam radius in both $x$ and $y$ direction as shown in Figs. 4.5.1(b) and 4.5.1(d). Therefore, simple Gaussian beam parameters can be used to approximate the behavior of the beams. In this case the far-field half-divergence angles $\theta$ can be calculated using [18]:

$$
\tan (\theta)=\frac{\lambda}{\pi w_{0}}
$$

With the micro-ball lenses placed at $d=58 \mu \mathrm{m}, d=65 \mu \mathrm{m}$, and $d=72 \mu \mathrm{m}$, the obtained far-field half-divergence angles are $2.00^{\circ}, 1.56^{\circ}, 2.83^{\circ}$ at a wavelength of $1306 \mathrm{~nm}$ and $2.28^{\circ}, 1.85^{\circ}, 3.17^{\circ}$ at a wavelength of $1544 \mathrm{~nm}$, respectively. To compare these results to the case in which no micro-ball lens is used, Fig. 4.5.2 shows also a calculated beam radius for the case where the beam is coupled out from the waveguide without a micro-ball lens. In this case, the half-divergence angle is $13.2^{\circ}$ at a wavelength of $1306 \mathrm{~nm}$ and $12.4^{\circ}$ at a wavelength of $1544 \mathrm{~nm}$. The divergence of these beams are so large that the beam size is larger than our knife-edge detector at the shortest possible measurement distance, and as such no accurate measurement of the divergence angle of these beams could be performed. 


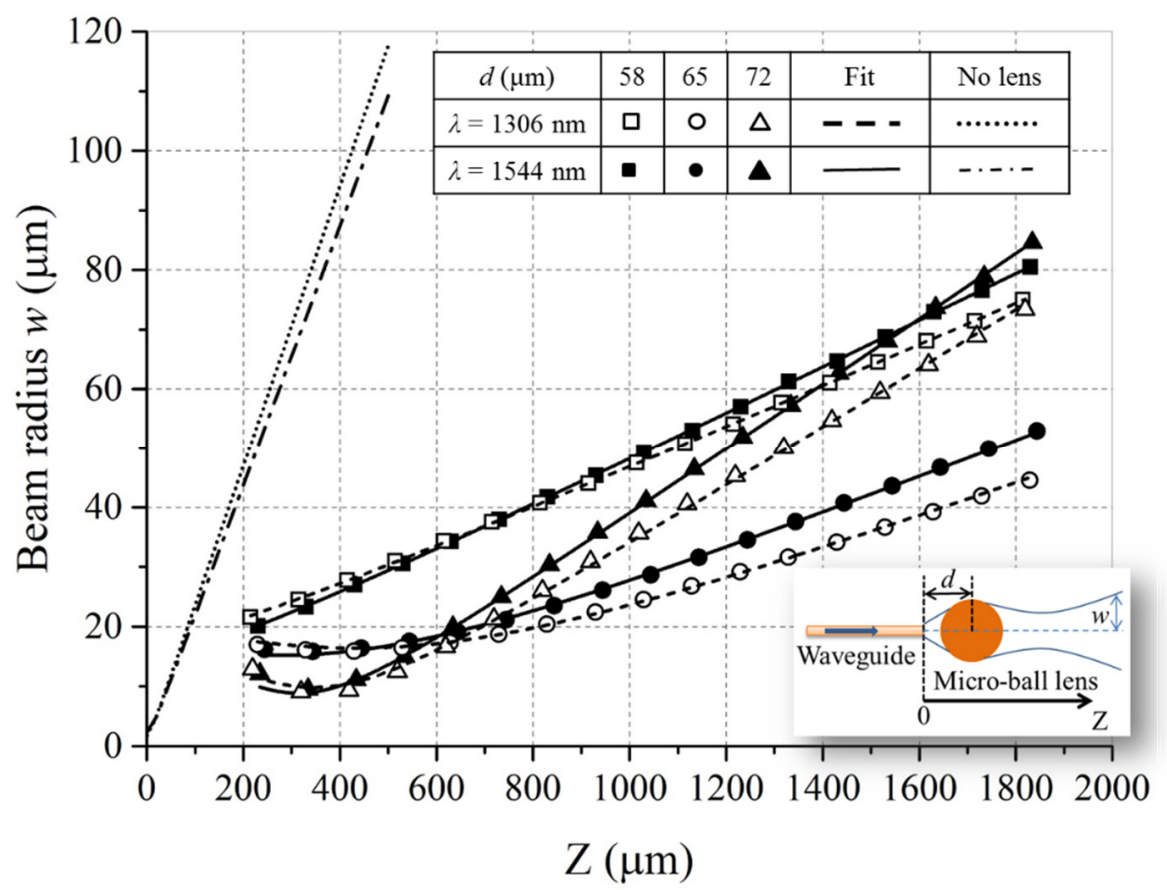

Fig. 4.5.2. Beam radius ( $1 / e^{2}$ radius) measurements in $x$ direction for different waveguide-lens distances with a knife-edge setup. $z$ is the beam propagation direction and $z=0$ is the end facet of the waveguide. The distance between the waveguide end facet and the lens center is $d$. The dot line and the dash dot line are the simulated beam size from a waveguide without a micro-ball lens at the wavelength of $1306 \mathrm{~nm}$ and $1544 \mathrm{~nm}$, respectively. The corresponding half divergence angle of these beams are $13.2^{\circ}$ and $12.4^{\circ}$. The lines goes thorough the measured data points are the corresponding fitted results.

The experimental results shown in Fig. 4.5.1 and Fig. 4.5.2 demonstrate that the microball lens can strongly reduce the divergence angle of light emerging from a waveguide in both horizontal and vertical directions. Obviously, the beam divergence can be varied within certain limits by changing the distance between the lens and the waveguide end facet in the mask design. Small differences in the beam shape (with the lens) are measured at two different wavelengths $(1306 \mathrm{~nm}$ and $1544 \mathrm{~nm}$ ). This chromatic aberration is caused by the mode shape difference in the waveguide and the dispersion of the lens material (photoresist). This chromatic aberration is expected not to be significant for our SS-OCT system that operates at a wavelength range of $92 \mathrm{~nm}$ centered at $1312 \mathrm{~nm}$ (see Section 5.2). The exact influence of the chromatic aberration on the OCT signal needs further study.

The optical loss of the micro-ball lens is simulated to be $11 \%$ due to Fresnel reflection, which is confirmed by experiment. 


\subsection{Summary}

In this chapter, we have demonstrated the design, fabrication and characterization of an integrated micro-ball lens system that allows for a significant reduction of the divergence angle, in both horizontal and vertical direction, of a light beam radiated from the end facet of an optical chip. The beam half divergence angle is reduced from $13.2^{\circ}$ to $1.56^{\circ}$ at a wavelength of $1306 \mathrm{~nm}$ and from $12.4^{\circ}$ to $1.85^{\circ}$ at a wavelength of $1544 \mathrm{~nm}$. The fabrication procedure allows careful tuning of the physical dimensions of the lens. In principle, the fabrication procedure is suitable for mass production, however the current yield of $85 \%$ needs to be improved. Furthermore, the fabrication strategy is not limited to the SiON waveguide platform as used here. It can be anticipated that, by carefully choosing suitable etching recipes for the fabrication of the platform and the right adhesion material in between the photoresist and the substrate, the strategy described here can be applied to waveguide technologies that are based on other materials. 


\section{References}

1. Akca, B.I., B. Považay, A. Alex, K. Wörhoff, R.M. de Ridder, W. Drexler, and M. Pollnau, Miniature spectrometer and beam splitter for an optical coherence tomography on a silicon chip. Optics Express, 2013. 21(14): p. 16648-16656.

2. Yurtsever, G., N. Weiss, J. Kalkman, T.G. van Leeuwen, and R. Baets, Ultra-compact silicon photonic integrated interferometer for swept-source optical coherence tomography. Optics Letters, 2014. 39(17): p. 5228-5231.

3. Ismail, N., L.P. Choo-Smith, K. Wörhoff, A. Driessen, A.C. Baclig, P.J. Caspers, G.J. Puppels, R.M. de Ridder, and M. Pollnau, Raman spectroscopy with an integrated arrayed-waveguide grating. Optics Letters, 2011. 36(23): p. 4629-4631.

4. Wei, C.L., F. Groen, M.K. Smit, I. Moerman, P. VanDaele, and R. Baets, Integrated optical elliptic couplers: Modeling, design, and applications. Journal of Lightwave Technology, 1997. 15(5): p. 906912.

5. Ismail, N., B.I. Akca, F. Sun, K. Wörhoff, R.M. de Ridder, M. Pollnau, and A. Driessen, Integrated approach to laser delivery and confocal signal detection. Optics Letters, 2010. 35(16): p. 2741-2743.

6. Gmachl, C., Plasmonics - A sharper approach. Nature Photonics, 2008. 2(9): p. 524-525.

7. Mitomi, O., K. Kasaya, and H. Miyazawa, Design of a single-mode tapered waveguide for low-loss chip-to-fiber coupling. Quantum Electronics, IEEE Journal of, 1994. 30(8): p. 1787-1793.

8. Van Laere, F., T. Claes, J. Schrauwen, S. Scheerlinck, W. Bogaerts, D. Taillaert, L. O'Faolain, D. Van Thourhout, and R. Baets, Compact focusing grating couplers for silicon-on-insulator integrated circuits. Ieee Photonics Technology Letters, 2007. 19(21-24): p. 1919-1921.

9. Cheung, Y.M. and C.H. Yiu, Simulation of the alignment sensitivity on the coupling efficiency of a ball-lens capped TO-Can laser diode source into a single-mode fiber. Proceedings of the 4th International Symposium on Electronic Materials and Packaging, 2002: p. 197-203.

10. Ratowsky, R.P., L. Yang, R.J. Deri, K.W. Chang, J.S. Kallman, and G. Trott, Laser diode to singlemode fiber ball lens coupling efficiency: Full-wave calculation and measurements. Applied Optics, 1997. 36(15): p. 3435-3438.

11. Wilson, R.G., Ball-lens coupling efficiency for laser-diode to single-mode fiber: comparison of independent studies by distinct methods. Applied Optics, 1998. 37(15): p. 3201-3205.

12. Chien, C.H., C.T. Pan, C.C. Hsieh, C.M. Yang, and K.L. Sher, A study of the geometry of microball lens arrays using the novel batch-fabrication technique. Sensors and Actuators a-Physical, 2005. 122(1): p. 55-63.

13. Shen, S.C. and J.C. Huang, Rapid fabrication of a micro-ball lens array by extrusion for optical fiber applications. Optics Express, 2009. 17(15): p. 13122-13127.

14. MicroChemicals. thick resist processing. [cited 2015 01-04-2015]; Available from: http://www.microchemicals.com/technical information/thick_resist_processing.pdf.

15. Lehar, O.P., M.A. Spak, S. Meyer, R.R. Dammel, C.J. Brodsky, and C.G. Willson. Resist rehydration during thick film processing. in SPIE. 2001.

16. Han, C.H., M.H. Seo, S.D. Ko, Y.H. Yoon, G.W. Yoon, and J.B. Yoon, Unconventional Use of a Photoresist as a Nitrogen Gas Generator Forming Transparent Dome-Shaped Microcavities. Advanced Engineering Materials, 2015.

17. Díazuribe, R., M. Roseteaguilar, and R. Ortegamartínez, Position Sensing of a Gaussian-Beam with a Power-Meter and a Knife Edge. Revista Mexicana De Fisica, 1993. 39(3): p. 484-492.

18. Svelto, O., Principles of Lasers. 2010: Springer. 


\section{Chapter 5}

\section{Single channel OCT measurements}

\subsection{Introduction}

In this chapter, we demonstrate the performance of our chip-based single channel sweptsource OCT (SS-OCT). In Section 5.2, we first demonstrate the performance of the chipbased SS-OCT without a micro-ball lens. Then, in Section 5.3, we demonstrate the signal improvement of the chip-based system with our integrated micro-ball lens. In these two sections we will only focus on the signal analysis. Since OCT imaging quality not only depends on the signal but also on the noise of the system, a detailed discussion of the noise and signal-to-noise ratio (SNR) as observed for our OCT system is provided in Section 5.4. We compare the performance of the chip-based system with an existing fiber-based OCT system in Section 5.5. In Section 5.6, we demonstrate a phantom measurement with our chipbased system. Section 5.6 also demonstrates experimentally a deconvolution approach, as introduced in Section 2.3, to reduce the artifacts caused by additional reflections at the microball lens surfaces, which act as multiple reference mirrors.

\subsection{OCT signal without micro-ball lens}

In this section we present the performance of the chip-based common-path OCT system. The schematic layout of the SS-OCT system is shown in Fig. 5.2.1. Light emitted from the swept laser (Axsun Technologies, USA, $1312 \mathrm{~nm}$ center wavelength, $92 \mathrm{~nm}$ optical bandwidth, $20.9 \mathrm{~mW}$ output power, $50 \mathrm{kHz}$ repetition rate) travels through an optical isolator (OI) and is coupled to the chip through a fiber array unit (FAU) with a $250 \mu \mathrm{m}$ pitch. The splitting ratio of the Y junction has been measured to be between 48/52 and 52/48 in the wavelength range of $1170 \mathrm{~nm}$ to $1650 \mathrm{~nm}$ as shown in Fig. 3.4.9. The wavelength range is limited at the short

Parts of this chapter is accepted by Optics Express as: Chang, L., N. Weiss, T.G. van Leeuwen, M. Pollnau, R.M. de Ridder, K. Wörhoff, V. Subramaniam, and J.S. Kanger, Chip based common-path optical coherence tomography system with an on-chip microlens and multi-reference suppression algorithm. 
wavelength side by the waveguide becoming multi-modal. The longest wavelength measured is limited by the available InGaAs detector. The in-coupled light propagates through the $\mathrm{Y}$ junction with approx. 50\% efficiency. The waveguide end facet, which back-reflects part of the light, acts as a reference plane as shown in Figs. 5.2.1(b) and Fig. 5.2.1(c). Due to the etching process, this facet is angled at 86 degrees $(\theta)$ with respect to the substrate plane, as shown in Fig. 5.2.1(c). Only $60 \%$ (simulated with a beam propagation method [1]) of the Fresnel-reflected light $(3.5 \%)$ couples to a guided waveguide mode, resulting in a calculated effective power reflection of $2 \%$, which was experimentally verified. The light that is transmitted through the facet is incident onto the sample. Part of the light back-reflected from the sample is coupled into the waveguide and interferes with the light that is reflected by the end facet. This interference signal propagates through the $\mathrm{Y}$ junction where approx. 50\% enters into the detection branch. The output signal from the chip is coupled to a detector (Thorlabs PDB 450C, USA; only one port of this balanced photodetector is used) through the same FAU.

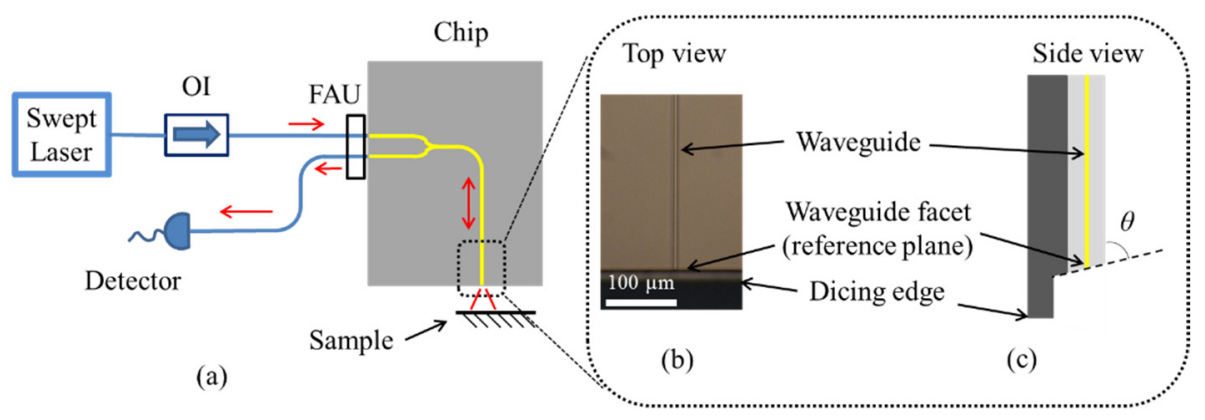

Fig. 5.2.1. Schematic of the SS-OCT experimental setup. (a) Light emitted by the swept laser source travels through an optical isolator (OI) and a fiber array unit (FAU) to the chip. The blue lines indicate optical fibers and the yellow lines represent the waveguides on the chip. (b) Microscope image of the end part of the chip showing the channel waveguide. (c) Schematic of the side view of the structure in Fig. 5.2.1(b). The end facet is angled at 86 degrees $(\theta)$ with respect to the Si substrate plane.

Details of the detector configurations are listed below:

- The average responsivity $\rho$ of the detector over the wavelength range of the light source is $1 \mathrm{~A} / \mathrm{W}$.

- The number of spectral channels measured in a scan (spectrum) is $M=1088$. All these channels are equally sampled in $k$ (wavenumber) space.

- The integration time $\tau_{i}$ of each spectral channel is $13.4 \mathrm{~ns}$ in average. This is calculated with $50 \mathrm{kHz}$ repetition rate of the laser (50000 scans in a second), 1088 spectral channels are measured within one scan and the duty cycle of the detector is 0.73 . There are small differences in the integration time from channel to channel due to the combination of the laser scanning and $k$ trigger of the detector, which is not significant in this study. 
A mirror sample is used to characterize the performance of this system. Fig. 5.2.2(a) shows the measured interference spectrum of the mirror sample at $100 \mu \mathrm{m}$ distance from the waveguide facet. Since we are not interested in the DC-part of the spectrum, the mean value of the original spectrum is subtracted in order to reduce the side lobes of the DC-part in the Fourier transform to obtain an A-scan. (The more advanced side lobes suppression algorithm introduced later in Section 5.4 is not applied to the results presented in Section 5.2 and Section 5.3.)

The A-scan result of the mirror sample at a distance of $100 \mu \mathrm{m}$ from the waveguide facet is shown in Fig. 5.2.2(b). This A-scan is the Fourier transform of the interference signal in Fig. 5.2.2(a). Note that the interference fringes need to be processed as a function of wavenumber instead of wavelength in order to obtain a proper Fourier transform in terms of path length. In order to better determine the signal peak height and location, zero padding (zeros are added at the end of the spectrum; the number of zeros is 9 times the number of measured data points) is used to get smaller calculated sampling size in the optical path axis. The full width at half maximum (FWHM) of the signal peak is measured to be $12.0 \pm 0.2 \mu \mathrm{m}$, which is almost identical with the fiber-based system with the same light source and detector as discussed in Section 5.5.

The SNR is calculated using Equation (2.5.2)

$$
S N R_{d B}=20 \log _{10}\left(\frac{q_{p e a k}}{\sigma}\right)
$$

where $q_{\text {peak }}=705.4$ is the peak magnitude of the signal and $\sigma=0.52$ is the standard variation between $3 \mathrm{~mm}$ and $5 \mathrm{~mm}$ (an arbitrary selected region, which is far away from the signal peak) determined from the measurement in Fig 5.2.2. The SNR in this case is calculated to be $62.5 \mathrm{~dB}$. By increasing the number of averaged measurements, the SNR increases. The measurement shown in Fig. 5.2.2 is repeated with 1000 continuous scans, where the $S N R_{d B}$ are $52.71 \pm 0.39 \mathrm{~dB}, 60.90 \pm 0.36 \mathrm{~dB}, 64.39 \pm 0.24 \mathrm{~dB}$ and $64.9 \mathrm{~dB}$ respectively in the case of averaging over 1, 10, 100 and 1000 measurements. (No error bar is available for the average of 1000 measurements.) Based on our experience, the fiber-chip coupling efficiency uncertainty has the most influence to the uncertainty in the SNR. Since all these measurements are finished within $0.02 \mathrm{~s}$, the fiber-chip coupling efficiency is close to constant. Thus the error bars in these $S N R_{d B}$ are small. We use an averaging over 30 measurements, since very large number of averaging needs much longer imaging time and only slightly improves the SNR. The shot noise limited $S N R_{d B}$ without averaging is $83 \mathrm{~dB}$, which is calculated with Equation (2.5.12) and the measured power listed in Table 5.3.1. 
(a)
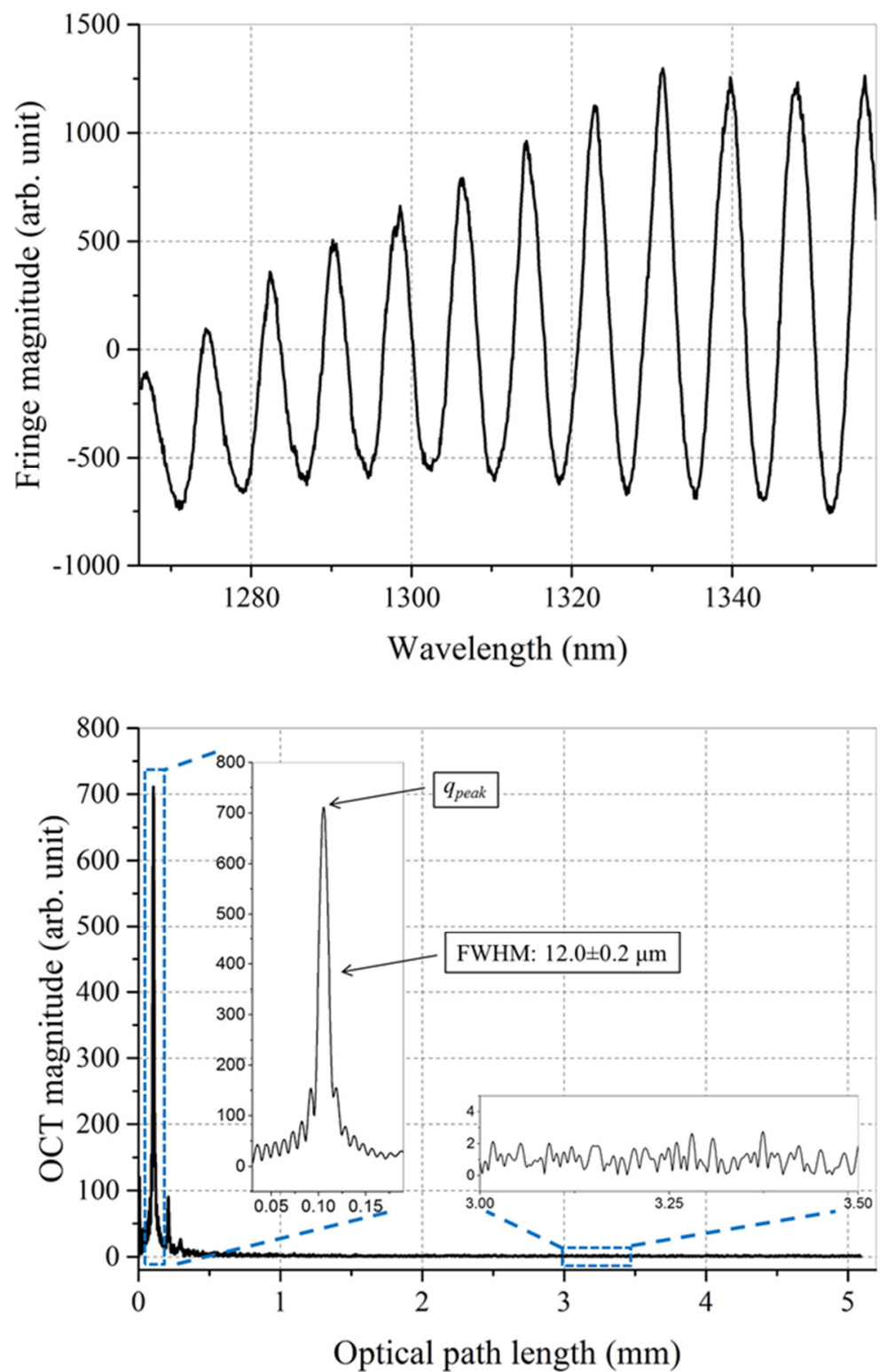

Fig. 5.2.2. (a) Interference fringes (with a mean value of 0 ) of a reflection from a mirror which is $100 \mu \mathrm{m}$ away from the waveguide facet. This interference fringes shown here is the result of an averaging over 30 measurements. (b) An A-scan, Fourier transform of the interference signal shown in (a). The horizontal axis is the optical path length between the waveguide facet and the sample reflecting surface. The vertical axis is the OCT magnitude which is proportional to the square root of the power reflected from the sample. 
The signal roll-off of this common-path OCT has been determined by measuring the signal peak magnitude for a mirror sample placed at different distances from the waveguide facet. The results are shown in Fig. 5.2.4. In some of the measurements, the light reflected twice between the mirror and the chip is still visible as a smaller peak compared with the main one (first reflection from the mirror). Therefore, the main peak position of each measurement is marked with a downward-pointing triangle. All triangles have been shifted up a little bit in order to visualize the measurement curve.

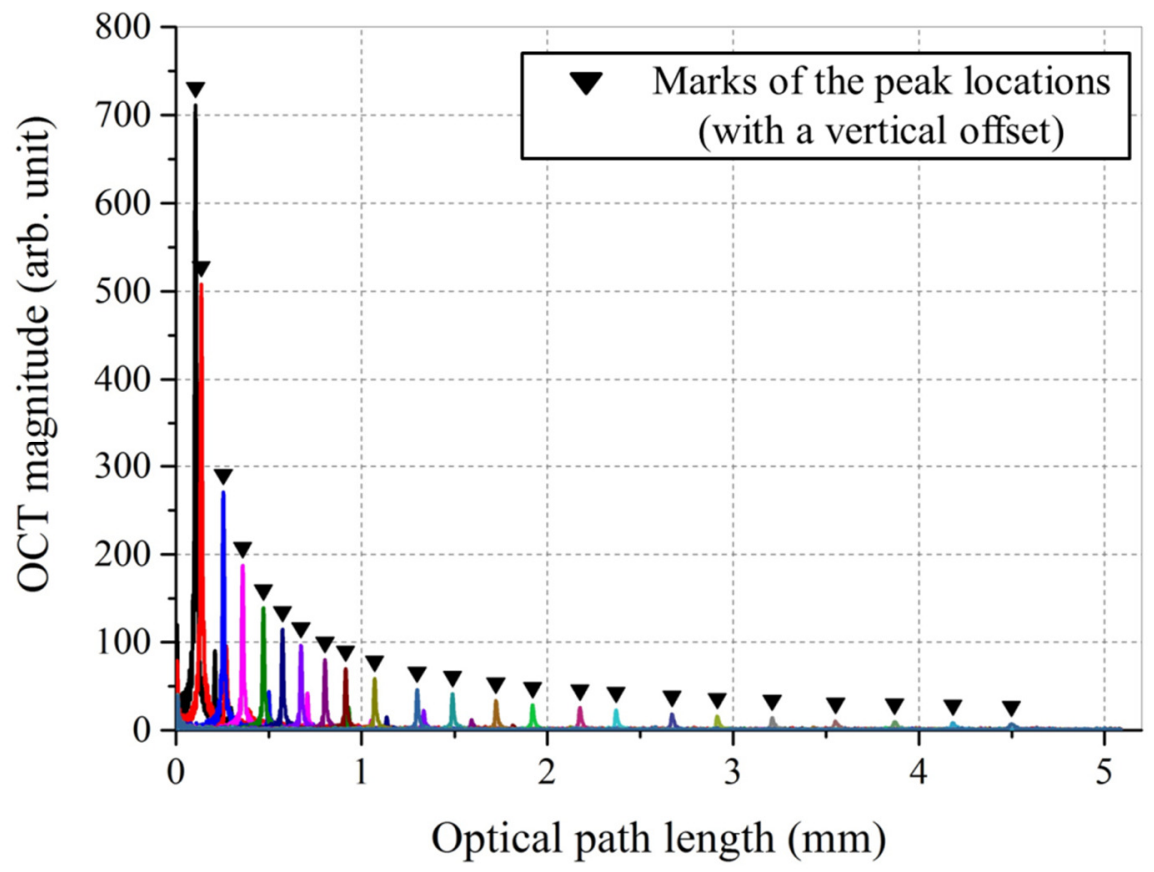

Fig. 5.2.4. Signal roll-off of a chip-based common-path OCT.

Due to the large divergence $\left(13.2^{\circ}\right.$ half divergence angle) of the light exiting the waveguide, the signal decreases very fast as the mirror moves away from the waveguide facet. This large divergence also lead to a poor lateral resolution. An integrated micro-ball lens is expected to provide a significant improvement in terms of lateral resolution and signal enhancement as will be shown in the next section. 


\subsection{OCT signal enhancement with an on-chip micro-ball lens}

In this section we present the performance of a chip-based common-path OCT system, which consists of one Y-junction and an integrated micro-ball lens. The design, fabrication and optical characterization of the integrated micro-ball lens were described in Chapter 4 . The presence of a lens is the only difference compared with the system described in Section 5.2. The schematic layout of this SS-OCT system, with a micro-ball lens, is shown in Fig. 5.3.1. The divergence reduction that is obtained by introducing the lens has been shown in Section 4.5. For the experiments reported here, we chose a micro-ball-lens arrangement (65 $\pm 0.5 \mu \mathrm{m}$ facet-to-lens-center distance, lens radius of $49 \pm 0.5 \mu \mathrm{m}$ ) providing the best possible collimated beam $\left(1.56^{\circ}\right.$ half divergence angle, $15.3 \mu \mathrm{m}$ beam waist at $0.3 \mathrm{~mm}$ optical path length, corresponding to an expected FWHM lateral resolution of $18 \mu \mathrm{m}, 29 \mu \mathrm{m}$, $57 \mu \mathrm{m}$ and $150 \mu \mathrm{m}$ at $0.3 \mathrm{~mm}, 1 \mathrm{~mm} 2 \mathrm{~mm}$ and $5 \mathrm{~mm}$ optical path length, respectively). The reduced beam divergence leads to a better lateral resolution and a higher chip-sample-chip coupling efficiency.

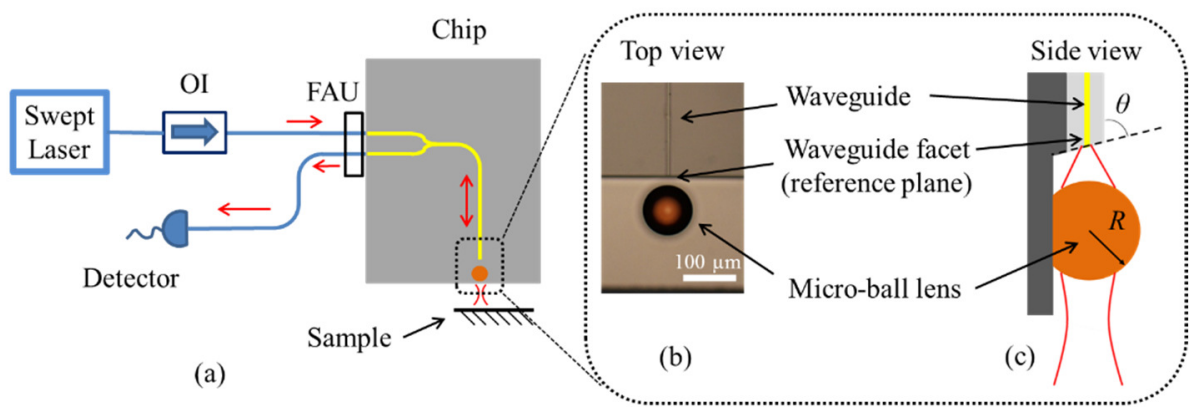

Fig. 5.3.1. Schematic of the SS-OCT experiment setup with a micro-ball lens. (a) Light emitted by the swept laser source travels through an optical isolator (OI) and a fiber array unit (FAU) to the chip. The blue lines indicate optical fibers and the yellow lines represent the waveguides on the chip. (b) Microscope image of the end part of the chip showing the channel waveguide and the micro-ball lens. (c) Schematic of the side view of the structure in Fig. 5.3.1(b). The end facet is angled at 86 degrees $(\theta)$ with respect to the Si substrate plane.

The optical power levels were measured at several positions in the optical setup without and with a microlens (Table 5.3.1). These optical power levels are important to quantitatively understand the signal enhancement realized with the micro-ball lens and for proper noise analysis. The laser power (9.0 $\pm 0.2 \mathrm{~mW}$ before coupling to the chip) was the same for all measurements discussed in Table 5.3.1. A mirror sample was located as close as possible to the chip, which is approx. $0.1 \mathrm{~mm}$ distance, resulting in $0.3 \mathrm{~mm}$ and $0.1 \mathrm{~mm}$ optical path length from the sample to the waveguide facet in the case with and without a lens, respectively. The reflected reference power $P_{R}$ and reflected sample power $P_{S}$ were both measured at the detector location as shown in Fig. 5.3.1(a). The power incident on the sample is measured by 
placing a power meter at the position of the mirror sample. The irradiance at the sample is estimated using the beam radius $(23.6 \pm 0.5 \mu \mathrm{m}$ without lens, $15.3 \pm 0.5 \mu \mathrm{m}$ with lens, see Section 4.5) at the sample position.

The results in Table 5.3.1 show that $P_{R}, P_{I}$, and the irradiance at the sample are similar in both systems. The slightly larger $P_{R}$ and slightly smaller $P_{I}$ in the situation with a microball lens are due to reflections from the surfaces of the lens. Despite the larger optical path length between sample and waveguide facet $P_{S}$ is approximately 100 times larger than compared to that without the lens. $P_{R}$ and $P_{I}$ are kept the same as in Table 5.3.1 for all the OCT measurements with the chip system in this thesis. $P_{S}$ and the irradiance at the sample are different for different optical path lengths. $P_{S}$ decreases rapidly in case of no lens, such that it cannot be measured directly (with the current setup) at larger optical path lengths but can only be calculated from the OCT measurements.

Table 5.3.1. Measured reflected reference power, reflected sample power and power incident on the sample with and without a micro-ball lens. Laser power before coupling to the chip is $9.0 \pm 0.2 \mathrm{~mW}$. The sample is a mirror.

\begin{tabular}{|c|c|c|}
\hline $\begin{array}{c}\text { Reflected reference power } \\
P_{R}(\mu \mathrm{W})\end{array}$ & Without micro-ball lens & With a micro-ball lens \\
\hline $\begin{array}{c}\text { Reflected sample power } \\
P_{S}(\mu \mathrm{W})\end{array}$ & $2.8 \pm 0.5$ & $14.5 \pm 0.5$ \\
\hline $\begin{array}{c}\text { Power incident on the sample } \\
P_{I}(\mathrm{~mW})\end{array}$ & $2.9 \pm 0.1$ & $2.6 \pm 0.1$ \\
\hline $\begin{array}{c}\text { Peak irradiance }(\text { Gaussian } \\
\text { beam) at the sample } \\
(\text { W/cm }\end{array}$ ) & $(3.3 \pm 0.3) \times 10^{2}$ & $(7.1 \pm 0.5) \times 10^{2}$ \\
\hline $\begin{array}{c}\text { Optical distance between the } \\
\text { mirror sample to the } \\
\text { waveguide end facet* } \\
(\mu \mathrm{m})\end{array}$ & $100 \pm 1$ & $320 \pm 1$ \\
\hline \multirow{2}{*}{ The reason of non-equal optical distance between these two measurements is discussed in the main text } \\
\hline
\end{tabular}


(a)

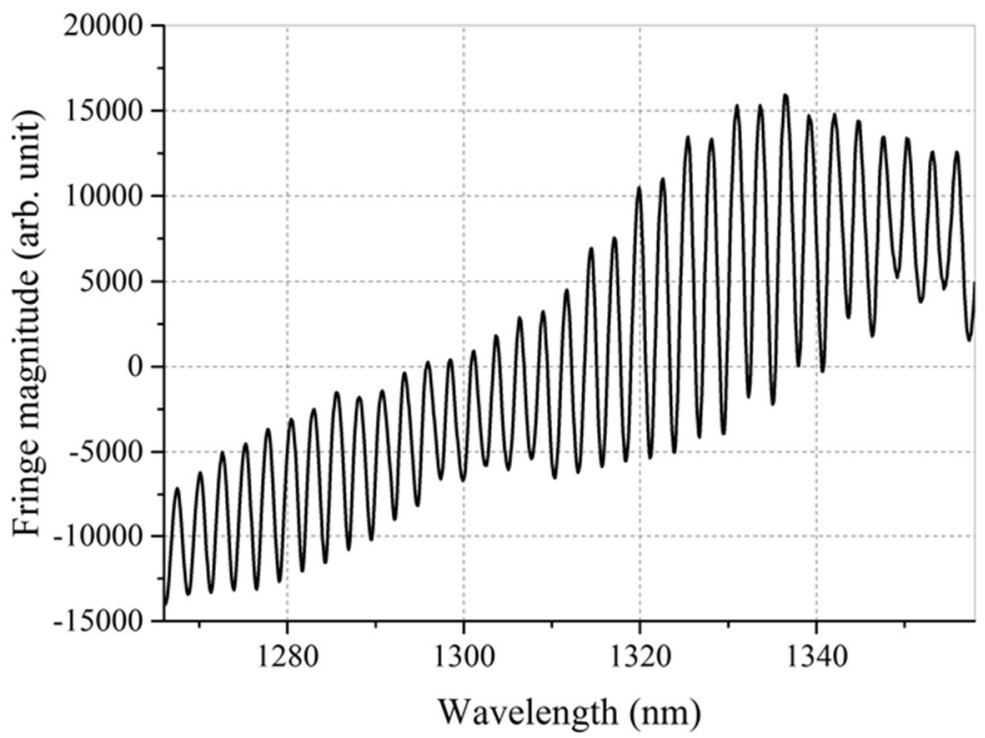

(b)

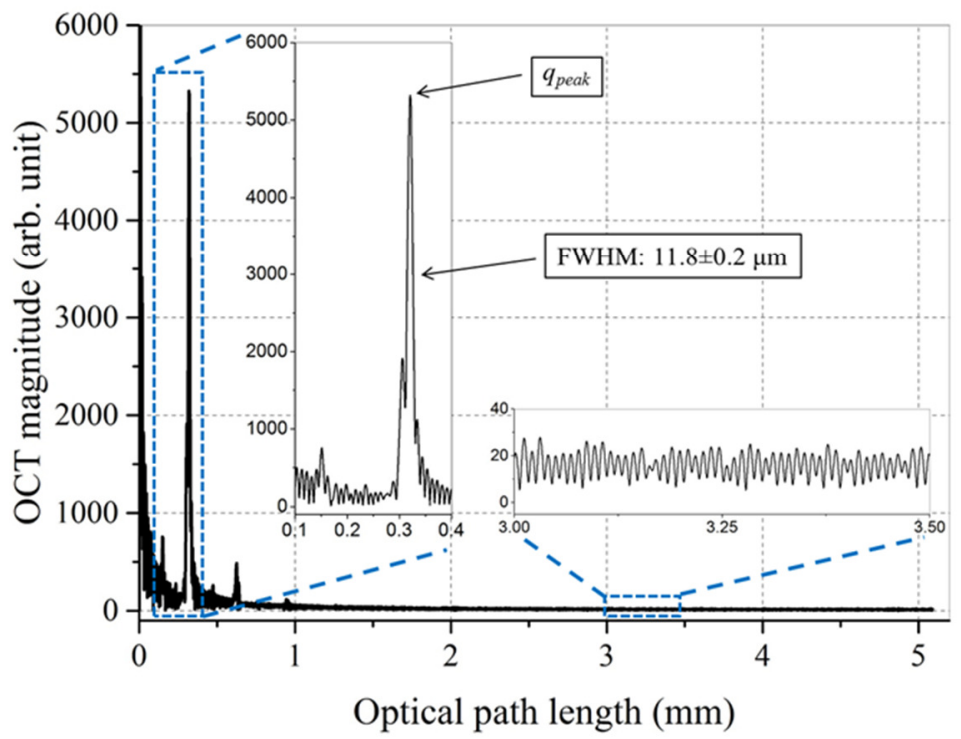

Fig. 5.3.2. (a) Interference fringes (with a mean value of 0 ) of a reflection from a mirror which is $320 \mu \mathrm{m}$ away from the waveguide facet measured with a micro-ball lens. This interference fringes shown here is the result of an averaging over 30 measurements. (b) An A-scan, Fourier transform of the interference signal shown in (a). The horizontal axis is the optical path length between the waveguide facet and the sample reflecting surface. The vertical axis is the OCT magnitude which is proportional to the square root of the power reflected from the sample. 
Figure 5.3.2(a) is a measured interference spectrum obtained with the chip system with a micro-ball lens, where a mirror sample is located $320 \mu \mathrm{m}$ away from the waveguide facet. The arb. unit is the same as in Fig. 5.2.2(a). This spectrum has a more complex envelope compared to the spectrum measured without a lens in Fig. 5.2.2(a). This is due to the effect of the additional reference surfaces, which will be discussed in more detail in Section 5.6. The amplitude of the modulation in this spectrum is also much larger than the one in Fig. 5.2.2(a).

The arb. unit in the A-scan shown in Fig. 5.3.2(b) is the same as in Fig. 5.2.2(b) and Fig. 5.2.4. The FWHM of the signal peak is measured to be $11.8 \pm 0.2 \mu \mathrm{m}$, which is almost the same as the one without a lens and also in good agreement with the bandwidth-limited resolution. The peak magnitude increased significantly compared with the peak measured without a micro-ball lens in Fig. 5.2.2(b).

The OCT signal roll-off (measured as the peak magnitude in the A-scan as a function of optical path length) with and without a micro-ball lens is compared in Fig. 5.3.3. For these measurements the mirror sample was positioned at different distances from the waveguide facet, with the same light source and detector settings in all cases. The presence of the microball lens enhances the signal strength by up to $37 \mathrm{~dB}$ depending on the optical path length. In the same figure, the calculated expected signal roll-off, $\mathfrak{R}_{\text {roll-off }}(z)$, is presented, following Equation (2) in the paper of Yun [2]

$$
\mathfrak{R}_{\text {roll-off }}(z)=\left(\frac{\sin z^{\prime}}{z^{\prime}}\right) \cdot \exp \left|-\frac{\psi^{2}}{2 \ln 2} z^{\prime 2}\right|,
$$

where $z^{\prime}=(\pi / 2) \cdot\left(z / z_{\text {MAX }}\right)$ denotes the optical path length, $z$, normalized to the maximum value of $z_{\text {MAX }}=5.1 \mathrm{~mm}$, and $\psi=0.73$ denotes the ratio of the spectral resolution to the spectral sampling interval which is approximated by the detector duty cycle $(0.73)$. For comparison, the calculated signal is normalized to the largest signal measured with a microball lens. The calculated signal roll-off is based on the finite spectral resolution of the system, assuming a constant chip-sample coupling efficiency over the whole optical path length range.

From Fig. 5.3.3 it shows that the micro-ball lens increases the $6 \mathrm{~dB}$ roll-off length from $0.2 \pm 0.1 \mathrm{~mm}$ to $1.7 \pm 0.1 \mathrm{~mm}$. Since we could not measure the sample signal at exactly zero optical path length in our common-path configuration, the $6 \mathrm{~dB}$ signal roll-off length is referred to the closest point (first point) measured in each system. The smaller signal roll-off length in the experiment, compared with the calculated one (which assumes a hypothetical $0^{\circ}$ divergence beam), is caused by the decrease of the chip-sample coupling efficiency due to the beam divergence. 
The roll-off curve may be tuned by designing the lens coupling efficiency. For example, a micro-ball lens provides a focus at $3 \mathrm{~mm}$, which leads to an improvement of the coupling efficiency in the middle of the roll-off curve. Thus, for a different lens the $6 \mathrm{~dB}$ roll-off length may be longer than the theoretical maximum of $3.1 \mathrm{~mm}$, shown in Fig. 5.3.3. The effect of size and position of micro-ball lens on the signal roll-off curve has not been studied here.

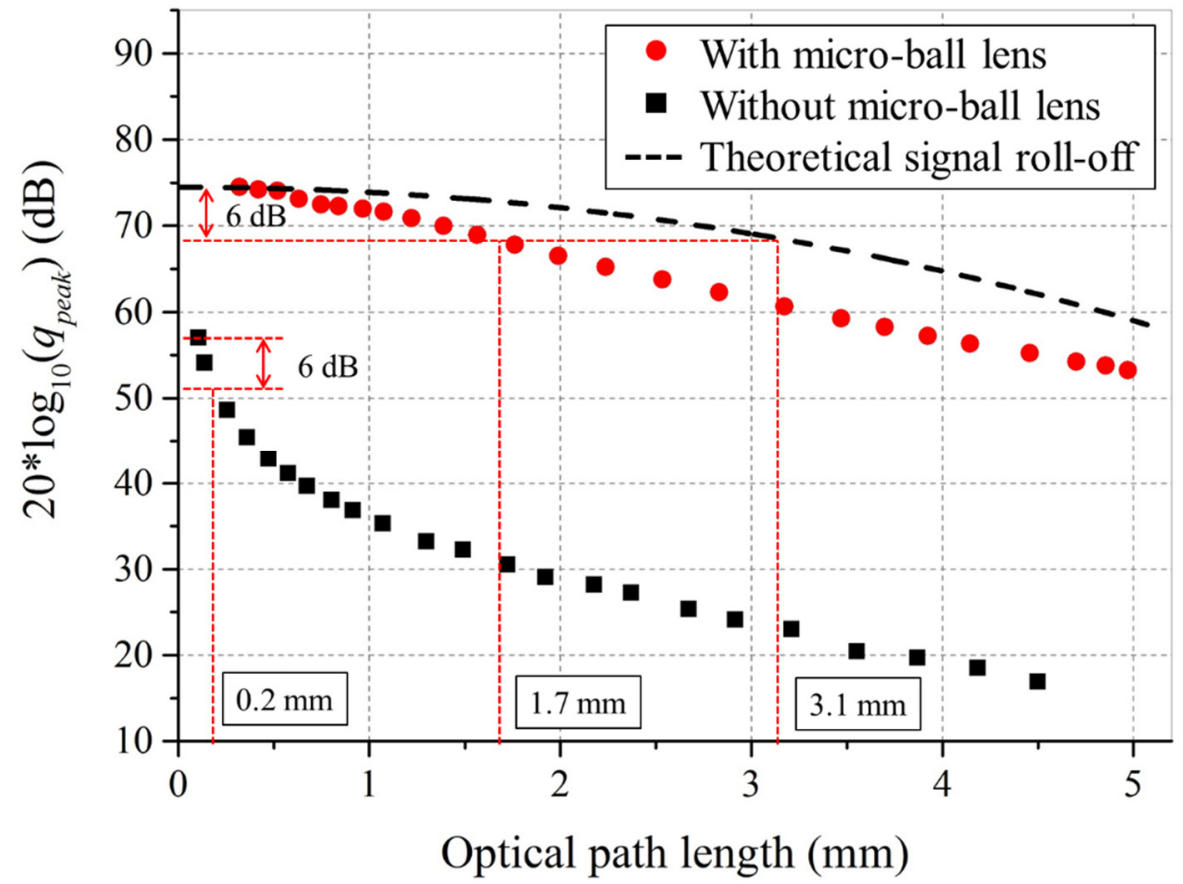

Fig. 5.3.3. Signal roll-off of the chip-based common-path OCT with and without a micro-ball lens. The peak position of each A-scan is marked with a red dot for the case with a lens and a black square for the case without lens. The peak magnitude is given in $\mathrm{dB}$ units, where $0 \mathrm{~dB}$ corresponds to the value 1 of the arbitrary unit used in Fig. 5.3.2(b). The dashed black curve is a calculated signal roll-off based on the finite spectral resolution of the system. The red dashed lines indicate the $6 \mathrm{~dB}$ roll-off optical path length of each curve.

\subsection{OCT noise and SNR arguments}

In Section 5.3, we have discussed the signal enhancement with a micro-ball lens and how the signal decreases with increasing of optical path length. However, for an OCT imaging system, the SNR is the key performance parameter. Therefore, a study of noise and SNR in our system is discussed in this section.

In Section 5.4.1, we will first discuss a technique, local mean removal, used to remove 
(or largely suppress) the largest noise source, side-lobe-noise, in the system. The side-lobenoise is not random noise, but an artefact caused by signal processing. We then present the details of calculating the local mean value of our data in Section 5.4.2. (This side-lobe-noise suppression algorithm is applied to all the OCT measurements in this thesis discussed after Section 5.4.2.) The noise that remains after the side-lobe-noise suppression is measured as function of optical path length for both chip systems (with and without a lens) in Section 5.4.3. The difference in the SNR roll-off of the two chip systems is discussed in Section 5.4.4.

\subsubsection{Suppression of the Fourier transform induced side-lobes with local mean removal}

In this section, we discuss the technique that is used to suppress the side-lobes in the A-scan that are caused by the Fourier transform of a truncated interference spectrum.

Fig. 5.4.1(a) shows an example of an interference spectrum, generated from a mirror sample, as measured by the chip system with a micro-ball lens. The A-scan result of this spectrum is shown in Fig. 5.4.2. The noise floor in this original A-scan result clearly has a periodic nature, as can be seen in the magnified inset of Fig. 5.4.2. This periodic behavior in the noise floor mainly originates from side lobes in the Fourier transform of a finite-length sample with discontinuities at the start and end of the data series.

A commonly used technique to reduce these side lobes is applying a windowing function before the Fourier transform. However, application of a windowing function will reduce the effective bandwidth of the spectrum and thus result in a broadening of the A-scan peak (lower axial resolution).

We use a different approach to reduce the amplitude of the side lobes with negligible influence on both signal amplitude and axial resolution. A windowing function reduces the amplitude of the side lobes by avoiding the sharp discontinuities at the two ends of the spectrum [3]. Our approach uses the same strategy. The first step is flattening the spectrum by subtracting its own local mean value, see Fig. 5.4.1(a). (The details of calculating the local mean value is introduced in Section 5.4.2.) The second step is truncating the resulting spectrum to the nearest zero crossings at both ends, see Fig. 5.4.1(b). The final spectrum, which we will refer to as the "local-mean-removed" spectrum, is shown with the red solid line in Fig. 5.4.1(b).

The A-scan result as determined using the original spectrum is compared to the A-scan as determined using the local-mean-removed spectrum (Fig. 5.4.2). The signal peaks (at $0.32 \mathrm{~mm}$ ) show a good overlap. The differences in both height and full width half maximum (FWHM) are less than 2\%. The noise floor (standard deviation) is decreased by approximately $70 \%$ of the original value, which leads to an increase of the SNR by approximately $10 \mathrm{~dB}$. 


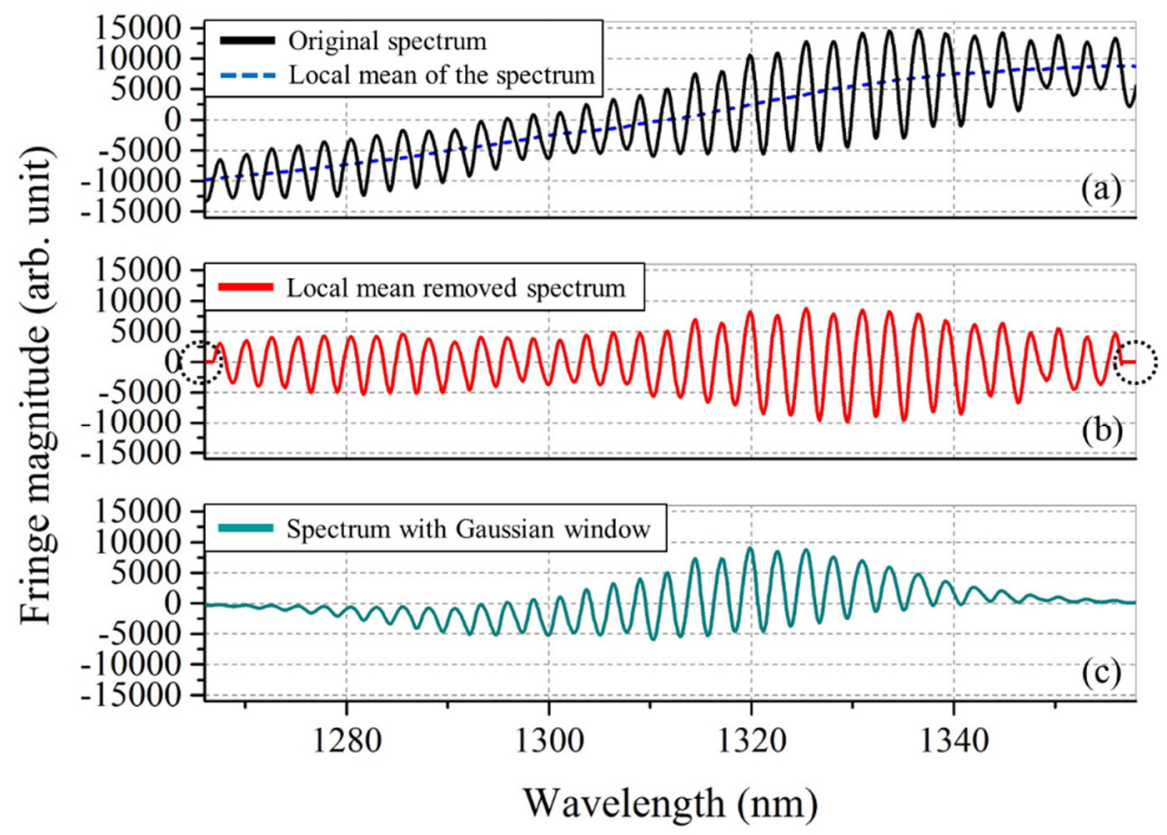

Fig. 5.4.1. Example of interference spectra processing. (a), (b) and (c) have the same $x$ and $y$ axes. The black solid line in (a) is the original interference spectrum (with a mean value of 0 ) of a reflection from a mirror sample measured with a chip having a micro-ball lens. The blue dashed line in (a) is the local mean of the original spectrum. The red solid line in (b) shows the spectrum after subtracting the local mean. The two ends of this local-mean-removed spectrum (marked with black dashed circles) are truncated to the nearest zero crossing. The cyan solid line in (c) shows the spectrum after applying a Gaussian windowing.

A commonly used Gaussian windowing function is applied and shown in Fig. 5.4.1(c) for comparison. The width of the Gaussian window is set such, that the SNR in the A-scan is the same as the local-mean-removed method. The Gaussian windowing A-scan result is shown in Fig. 5.4.2, and the main peak FWHM $(23.4 \pm 0.3 \mu \mathrm{m})$ is about two times larger than the one of local-mean-removed method $(11.8 \pm 0.2 \mu \mathrm{m})$.

In practice we observe that the method as introduced here reduces the noise floor in all measurements we have performed. The amount of reduction in the A-scan noise floor (varies from $-10 \mathrm{~dB}$ to $-1 \mathrm{~dB}$ ) depends very much on the magnitude of the side-lobes in the applied measurement. For example, in some of the measurements, the side-lobe-noise is much smaller than others due to, for instance, a flatter spectrum. Thus, the amount of noise floor reduced is much less than the ones with much more side-lobe-noise. On the other hand, the influence on the signal peak height and FWHM in all these measurements were found to be smaller than $2 \%$. The limitation of this method is that the local mean value contains low frequency modulation, determined by the size of the averaging window. Therefore, any signal 
due to features close to the zero optical path length will be suppressed or removed from the A-scan result. In the example given in Fig. 5.4.1 and Fig. 5.4.2, this suppression is clearly visible in the optical path length range between $0 \mathrm{~mm}$ to $0.2 \mathrm{~mm}$, where the suppression is stronger for path lengths closer to $0 \mathrm{~mm}$.

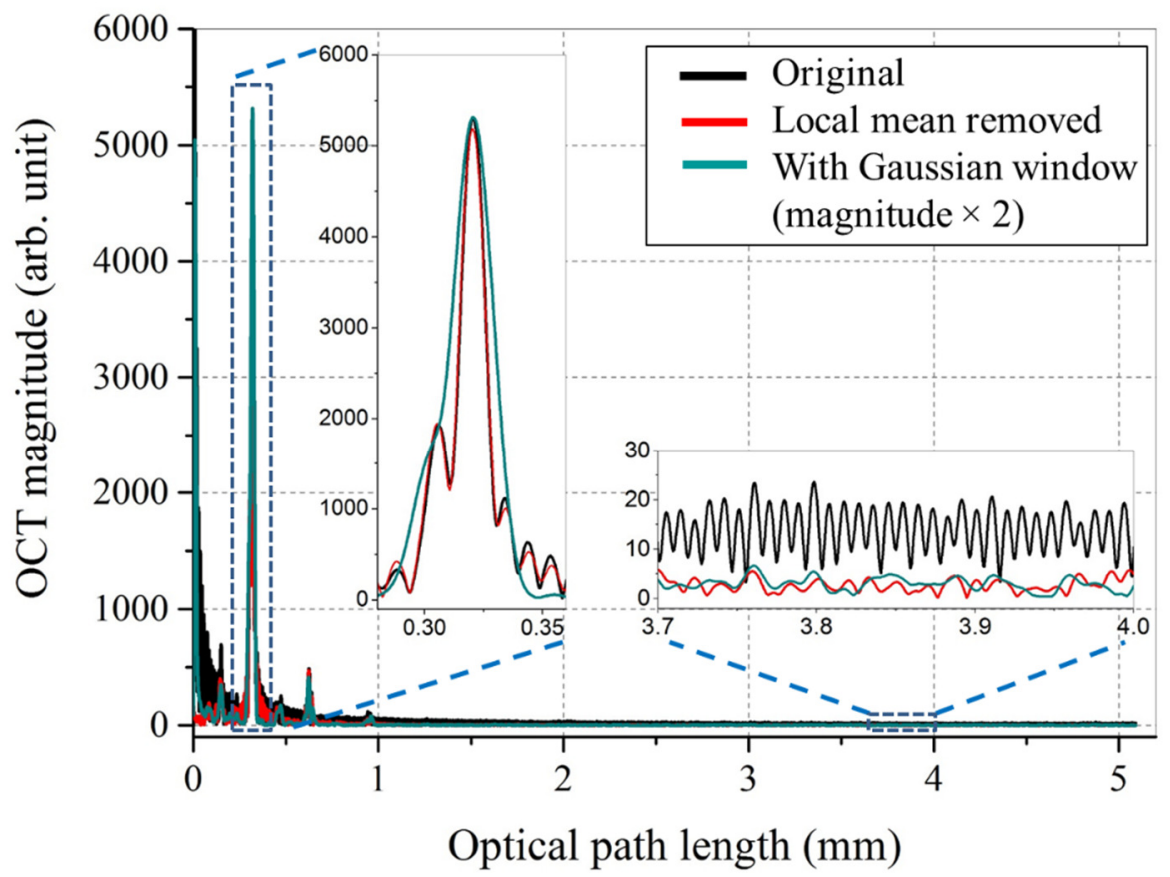

Fig. 5.4.2. The A-scans obtained from the spectra in Fig. 5.4.1. The black line is the A-scan result of the original spectrum (black curve in Fig. 5.4.1). The red line is the A-scan result of the local-mean-removed spectrum (red curve in Fig. 5.4.1). The cyan line is the A-scan result of the Gaussian windowed spectrum (cyan curve in Fig. 5.4.1).The inset graphs are zoomed in plots of the main signal peak and the noise floor.

\subsubsection{Local mean value estimation}

The OCT A-scan magnitude is related to the oscillation amplitude in the spectrum, thus the removed local mean value should be as smooth as possible to minimize the influence on the A-scan magnitude. There are many possible ways to estimate the local mean value of a curve. One of the ways is to use a moving average (MA), which calculates the unweighted mean of the previous $n$ data points (such as a Matlab function of 'movavg'). However the local mean resulting from this function ('movavg') itself is not smooth enough in many cases. There may be also large errors in the estimation of the local mean at the beginning of the data set, since $n-1$ zeros are added to the beginning of the data set by the 'movavg' function. There is also a delay effect depending on the MA window size $n$ which shifts the local mean value 
horizontally. In order to better estimate the local mean value, several additional steps are taken. The steps are summarized in Table 5.4.1 and the corresponding results are shown in Fig. 5.4.3.

Table 5.4.1. The steps of local mean value estimation

\begin{tabular}{|c|c|c|}
\hline Step & Operation & Note \\
\hline 1 & $\begin{array}{l}\text { Moving average on the original } \\
\text { spectrum from left to right with a } \\
\text { window size of } n_{1} \text { ( } 31 \text { points, } \\
\text { corresponding to } 31 \text { spectral } \\
\text { channels). }\end{array}$ & \multirow{2}{*}{$\begin{array}{l}\text { The choice of } n_{1} \text { and } n_{2} \text { are not completely } \\
\text { arbitrarily. They should be large enough to } \\
\text { smooth the lowest oscillation frequency of } \\
\text { interest in the spectrum. The sum of them } \\
\text { should be small to keep the linear extraction } \\
\text { region short enough, thus close to the real } \\
\text { local mean. They should not have common } \\
\text { divisor, except } 1 \text {, since the oscillation with a } \\
\text { period of their common divisor does not } \\
\text { smooth out sufficiently. }\end{array}$} \\
\hline 2 & $\begin{array}{l}\text { Moving average on the resulting } \\
\text { curve of step } 1 \text { from left to right } \\
\text { with a window size of } n_{2} \quad(59 \\
\text { points). }\end{array}$ & \\
\hline 3 & $\begin{array}{l}\text { Replace the error region (first } \\
n_{1}+n_{2} \text { points) in curve } 2 \text { by linear } \\
\text { extrapolation. }\end{array}$ & $\begin{array}{l}\text { This step may not necessary if the original } \\
\text { spectrum has a negligible local mean offset } \\
\text { from zero at the beginning. }\end{array}$ \\
\hline 4 & $\begin{array}{l}\text { Moving average on the original } \\
\text { spectrum from right to left with a } \\
\text { window size of } n_{1} \text { ( } 31 \text { points). }\end{array}$ & \multirow{2}{*}{$\begin{array}{l}\text { In practice, the original spectrum is flipped } \\
\text { horizontally to perform these MA, since the } \\
\text { 'movavg' function in Matlab only average } \\
\text { from left to right. }\end{array}$} \\
\hline 5 & $\begin{array}{l}\text { Moving average on the resulting } \\
\text { curve of step } 4 \text { from right to left } \\
\text { with a window size of } n_{2} \quad(59 \\
\text { points). }\end{array}$ & \\
\hline 6 & $\begin{array}{l}\text { Replace the error region in curve } 5 \\
\text { by linear extrapolation. }\end{array}$ & $\begin{array}{l}\text { This step may not necessary if the original } \\
\text { spectrum has a negligible local mean offset } \\
\text { from zero at the end. }\end{array}$ \\
\hline 7 & $\begin{array}{l}\text { Average of the resulting curve } \\
\text { from step } 3 \text { and } 6\end{array}$ & Compensate the delay effect in the MA. \\
\hline
\end{tabular}




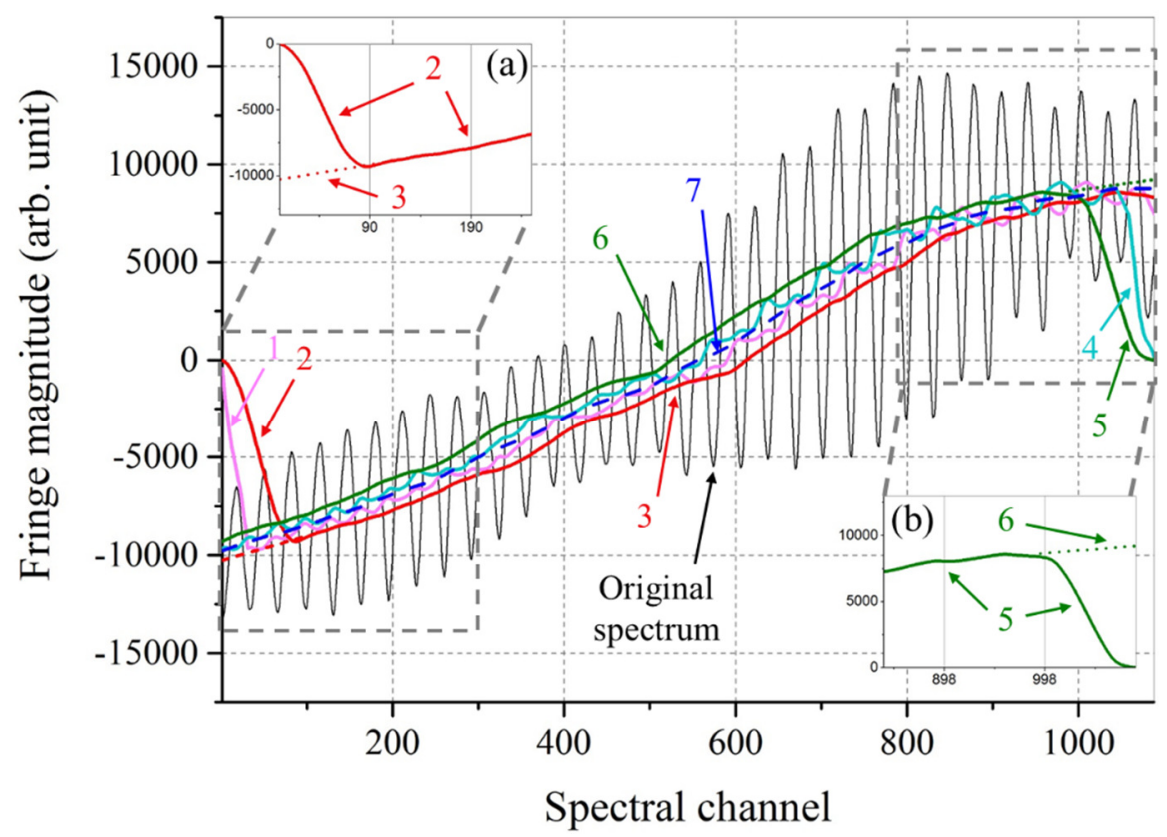

Fig. 5.4.3. Local mean value estimation. The curves indicated with numbers are the results of the corresponding steps in Table 5.4.1. Inset (a) and (b) show the linear extrapolation in step 3 and step 6, respectively. Curve 3 (or 6) is only different from curve 2 (or 5) because of the linear extrapolation at the beginning. The final estimated local mean curve is indicated with a blue dashed line marked with number 7 .

First, a cascade MA with different window sizes is very effective to smooth the local mean curve. The MA result has small oscillations as shown in curve 1 of Fig. 5.4.3 in case of a periodic spectrum (such as from a mirror sample). The amplitude of this oscillation depends on the relation of the MA window size $n$ and the period of the data. The minimum oscillation only happens when the window size is odd multiples of the half oscillation period of the spectrum. Increasing the window size is one way to suppress the oscillation in the local mean value. Based on our experience, a better way to smooth the local mean value curve (curve 2 of Fig. 5.4.3) is applying the MA again on curve 1 in Fig. 5.4.3 with a different window size.

Second, a linear extrapolation is used to reduce the local mean estimation error at the beginning of the spectrum. The Matlab function 'movavg' adds $n$ - 1 zeros at the beginning of the data set to fill up the 'previous $n$ data'. This leads to an error in terms of estimating the local mean at the beginning of the spectrum when the spectrum has a large local mean offset from zero at the beginning, as shown in curve 1 and curve 2 of Fig. 5.4.3. The error region is the sum of both window size $\left(n_{1}+n_{2}=31+59=90\right.$ points in our example $)$ in case of a two times cascade MA. In order to reduce the error in this region (first 90 data points), a linear extrapolation is used based on the curve between 90 and 190 data points as shown with curve 3 in Fig. 5.4.3. 
Third, delay effect in the MA is compensated by averaging the MA results of left-toright moving average and right-to-left moving average. (The right-to-left moving average is realized by flipping the spectrum horizontally in Matlab). All the steps in the left-to-right moving average (step 1-3 in Table 5.4.1) are repeated in the right-to-left moving average, thus the delay effect should be largely canceled out in their averaging.

This local mean estimation algorithm works for all the data measured with our system. The averaging window size and linear extrapolation region may change for different OCT system and different lowest interested oscillation frequency (shortest OCT sample distance). We did not have a detailed quantitative study about the relation between the averaging window size and the 'non-influenced' shortest OCT sample distance yet. (Since this algorithm works in our case, we did not spend more effort to investigate other possible algorithms.)

\subsubsection{OCT noise comparison}

The noise in an OCT measurement is specified as the standard deviation $\sigma$ of the signal fluctuations at the noise floor of an A-scan. After the suppression of the side-lobe-noise as described in Section 5.4.1, the remaining noise $\sigma$ is mainly consist of random measurement noise $\sigma_{\text {measured, }}$ which is given by

$$
\sigma \approx \sigma_{\text {measured }}=\sqrt{\sigma_{\text {read }}^{2}+\sigma_{\text {shot }}^{2}(P)+\sigma_{R I N}^{2}(P)},
$$

where $\sigma_{\text {read }}, \sigma_{\text {shot }}$, and $\sigma_{R I N}$ denote read-out noise, shot noise, and relative intensity noise (RIN), respectively, and $P=P_{R}+P_{S}$ is the total power on the detector. For given detector settings, $\sigma_{\text {read }}$ does not depend on the signal. Shot noise and RIN have power dependencies of $\sigma_{\text {shot }}(P) \propto \sqrt{P}$ and $\sigma_{R I N}(P) \propto P$, respectively [4].

The noise, $\sigma_{\text {measured }}$, measured as a function of the sample optical path length for the chip system, both with and without a lens is shown in Fig. 5.4.4. The noise is given in the same $\mathrm{dB}$ units as the signal in Fig. 5.3.3, i.e., $20 \log _{10}(\sigma)$. The noise stays constant with increasing optical path length in the chip system without a lens. The noise decreases with increasing optical path length in the chip system with a lens.

In both systems, the reference power is kept constant $(12.0 \mu \mathrm{W}$ without a lens and $14.5 \mu \mathrm{W}$ with a lens as shown in Table 5.3.1, and the sample power decreases with increasing optical path length. From the measured power data in Table 5.3.1, it can be seen that $P_{R} \gg P_{S}$ in the case without a lens. So the noise is dominated by the noise level of the reference power, where $\sigma_{\text {measured }} \approx \sqrt{\sigma_{\text {read }}^{2}+\sigma_{\text {shot }}^{2}\left(P_{R}\right)+\sigma_{R I N}^{2}\left(P_{R}\right)}$ from Equation (5.4.1) is a constant. This explains the constant noise level in the chip system without a lens. 


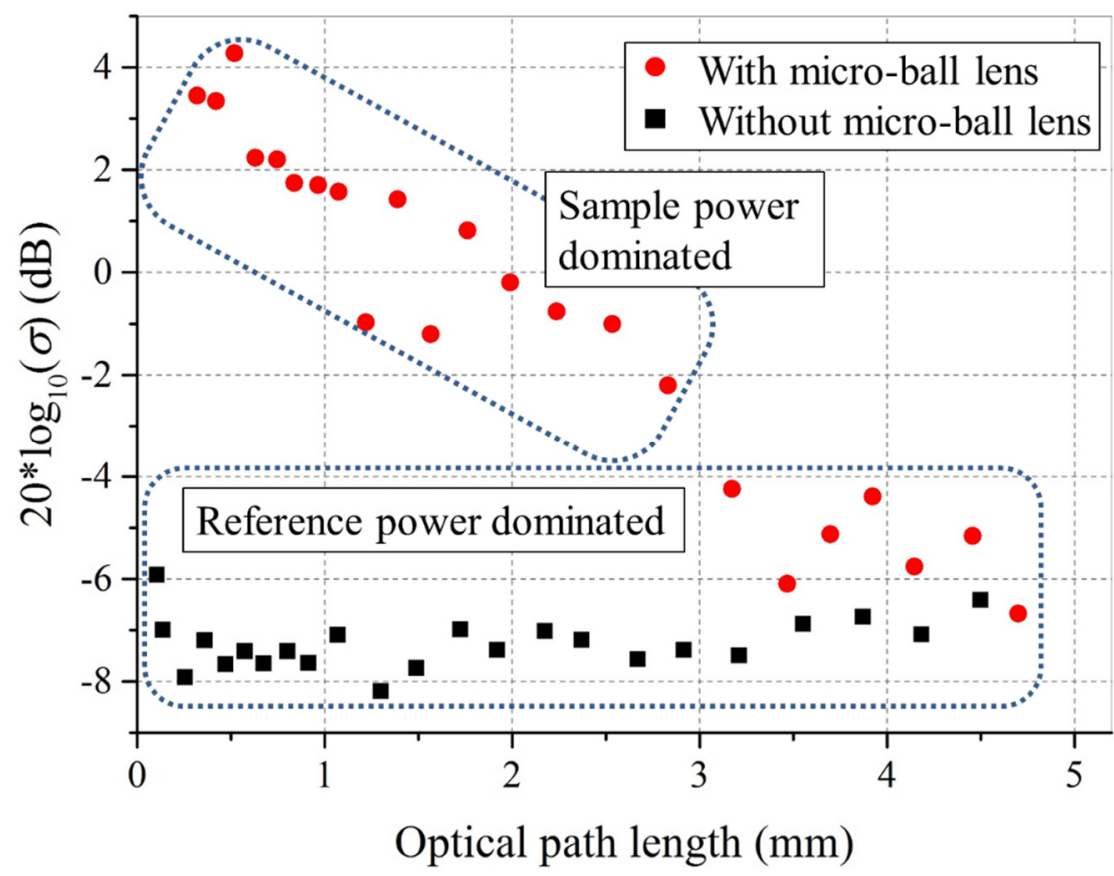

Fig. 5.4.4. Measured noise as a function of optical path length. The measurements are categorized into two groups, namely sample power dominated and reference power dominated.

The ratio of reflected sample power to reference power in the chip system with a lens can be calculated using Equation (2.5.9) (know $\sqrt{P_{R} P_{S}} \propto q_{\text {peak }}$ ), Fig. 5.3.3 (know every $q_{\text {peak }}$ ), and Table 5.3.1 (know the $P_{R}$ and $P_{S}$ of the largest $q_{\text {peak }}$ measured by both chip systems, with and without lens). The result is that the reflected sample power is a factor of 20 larger than the reference power $\left(P_{R}<<P_{S}\right)$ for a mirror-to-chip distance of $0.3 \mathrm{~mm}$, and decreases to $20 \%$ of the reference power $\left(P_{R} \gg P_{S}\right)$ at a distance of $4.7 \mathrm{~mm}$. At $3 \mathrm{~mm}$ optical path length, the reflected sample power is about the same as the reference power $\left(P_{R} \approx P_{S}\right)$. Therefore, the noise of the chip with a lens can be classified in two different regimes, as indicated in Fig. 5.4.4, namely the sample-power-dominated and the reference-power-dominated regimes.

In the measurements discussed above, the sample was a mirror causing a relatively high reflected sample power due to its high reflection coefficient. This condition is relevant in case of certain industrial applications where highly reflective surfaces are measured [5]. In case of biological applications, the tissue will produce considerably less sample power $\left(P_{R} \gg P_{S}\right)$, so that all measurements in this case would be performed in the reference-powerdominated regime with an approximately constant noise level. For any other partially reflecting samples [6-8], the noise in the chip system with a lens should fall in the region between the red dots and black squares indicated in Fig. 5.4.4. 


\subsubsection{SNR comparison}

The SNR is calculated with Equation (5.2.1). The SNR roll-off of with optical path length is shown in Fig. 5.4.5. The 6-dB SNR roll-off optical path length of the chip without a lens is $0.2 \pm 0.1 \mathrm{~mm}$, which is the same as its signal roll-off. However, the 6-dB SNR roll-off optical path length of the chip with a lens, in the sample-power-dominated regime, is $3.0 \pm 0.5 \mathrm{~mm}$ which is much larger than its signal roll-off $(1.7 \pm 0.1 \mathrm{~mm})$. This extended roll-off optical path length is due to the fact that the noise is decreasing with increasing optical path length as shown in Fig. 5.4.4.

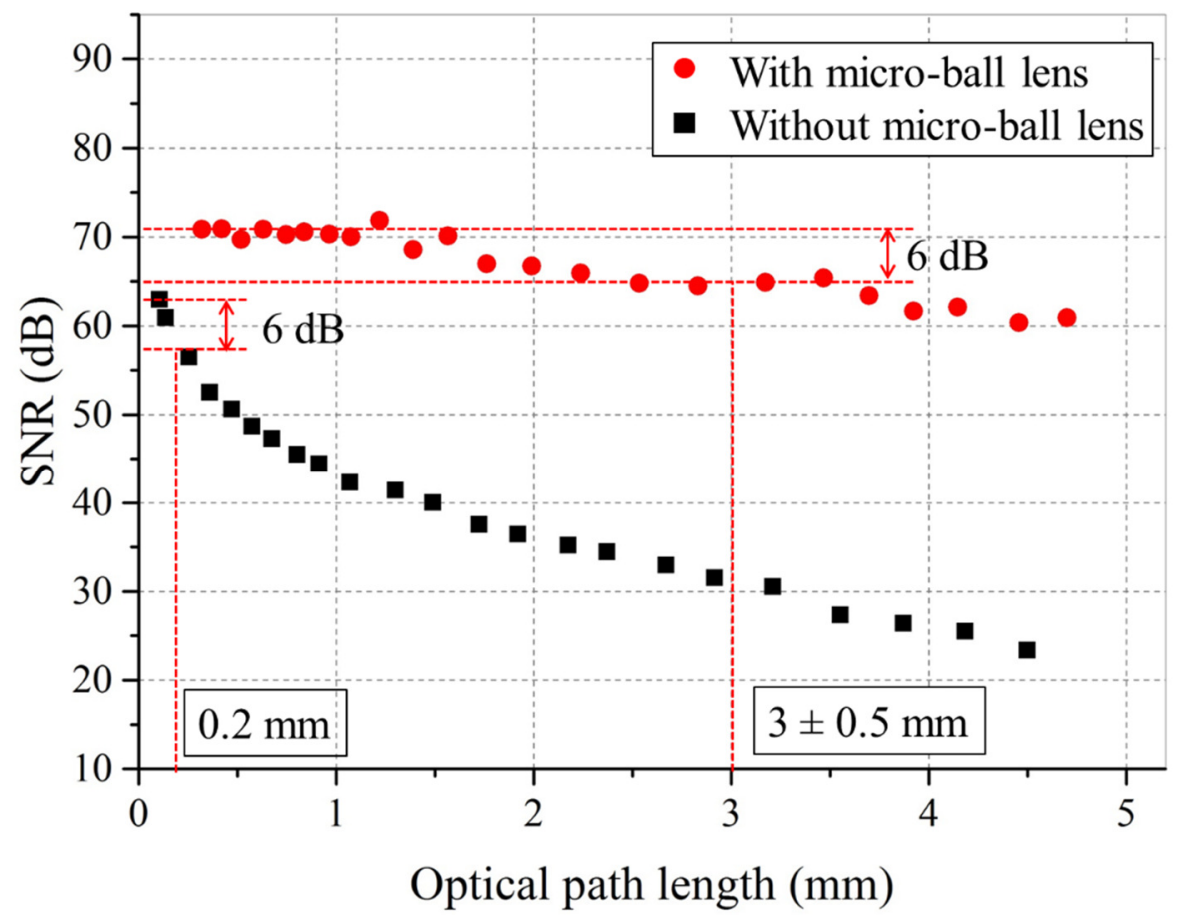

Fig. 5.4.5. SNR roll-off comparison between chips with and without a lens. The red dashed lines indicate the $6 \mathrm{~dB}$ roll-off optical path length of each curve. The SNR roll-off curve in the case with a lens is rather flat, leads to a large uncertainty $( \pm 0.5 \mathrm{~mm})$ in the $6 \mathrm{~dB}$ roll-off range.

The signal roll-off curve (Fig. 5.3.3) and the SNR roll-off curve (Fig. 5.4.5) together set the boundary of roll-off rate in the SNR for different samples. The SNR roll-off curve in Fig. 5.4.5 is measured with a mirror which has the largest sample power noise. This leads to the fastest decrease in the noise with sample power, thus the slowest decrease in the SNR. In the case of a weakly scattering sample, the noise will be dominated by a constant reference power noise, thus the SNR decreases at the same rate as the signal. For any other samples, the SNR roll-off is slower than the signal roll-off and faster than the SNR roll-off measured 
with a mirror. Thus the $6 \mathrm{~dB}$ SNR roll-off optical path length of any sample should be between $1.7 \pm 0.1 \mathrm{~mm}$ and $3.0 \pm 0.5 \mathrm{~mm}$ for the chip with a lens. For a weakly scattering tissue sample, the 6-dB SNR roll-off optical path length should be $1.7 \pm 0.1 \mathrm{~mm}$ (the same as the signal roll-off).

\subsection{Performance comparison between a chip system with a}

\section{micro-ball lens and a fiber system}

In this section, the performance of the chip system is compared to that of a bulk fiber system in the Biomedical Engineering and Physics group, Academic Medical Center, University of Amsterdam. The bulk fiber system is described in the paper of Weiss et al [9]. The following conditions were kept constant in the experiments:

- Settings of the swept laser source and the detector.

- Reflected reference power, $P_{R}=14.5 \pm 1 \mu \mathrm{W}$, in the absence of the sample.

- Power incident on the sample $P_{I}=2.6 \pm 0.1 \mathrm{~mW}$.

- Mirror sample placed at approx. $0.3 \mathrm{~mm}$ (optical path length) distance from the reference plane (the waveguide facet in case of the chip system).

The measured spectra are shown in Fig. 5.5.1. The fiber system has a larger fringe amplitude compare with the chip system. Since the sample in the fiber system was $20 \mu \mathrm{m}$ farther away from the reference plane than in the chip system, the spectra in Fig. 5.5.1 show slightly different periods for the two systems. The key parameters in the corresponding A-scans of both systems are listed in Table 5.5.1.

The OCT signal peak magnitude of the chip system is approximately one third of that of the fiber system and the FWHM of the signal peaks in both systems are comparable. The smaller signal peak magnitude in the chip system is caused by the larger optical losses compared to the fiber system. The power transmittance from the sample to the detector in the fiber system is measured to be $54 \pm 2 \%$ at this optical path length. This is mainly limited by the coupling efficiency of the bulk lens used in this fiber system. Based on Equation (2.5.9), the chip system should have approx. 9 times smaller transmittance $(\sim 6 \%)$ in the same path than in the fiber system. The power transmittance of every component from sample to detector in the chip system is as follows:

1. The micro-ball lens has a measured power transmittance of $89 \pm 2 \%$ due to Fresnel reflection.

2. The coupling from the micro-ball lens to the waveguide is $48 \pm 5 \%$, which is calculated based on the measured total transmittance at this optical path length and transmittance of every other components. This coupling efficiency is similar to that 
of the bulk lens used in the fiber system.

3. The propagation losses of the waveguide $(0.1 \mathrm{~dB} / \mathrm{cm}[10])$ lead to a transmittance of $91 \pm 2 \%$.

4. The on-chip Y junction has a transmittance into the detector arm of $49 \pm 2 \%$.

5. The coupling from the chip to the detector is $59 \pm 2 \%$ due to the mode mismatch between waveguide and fiber.

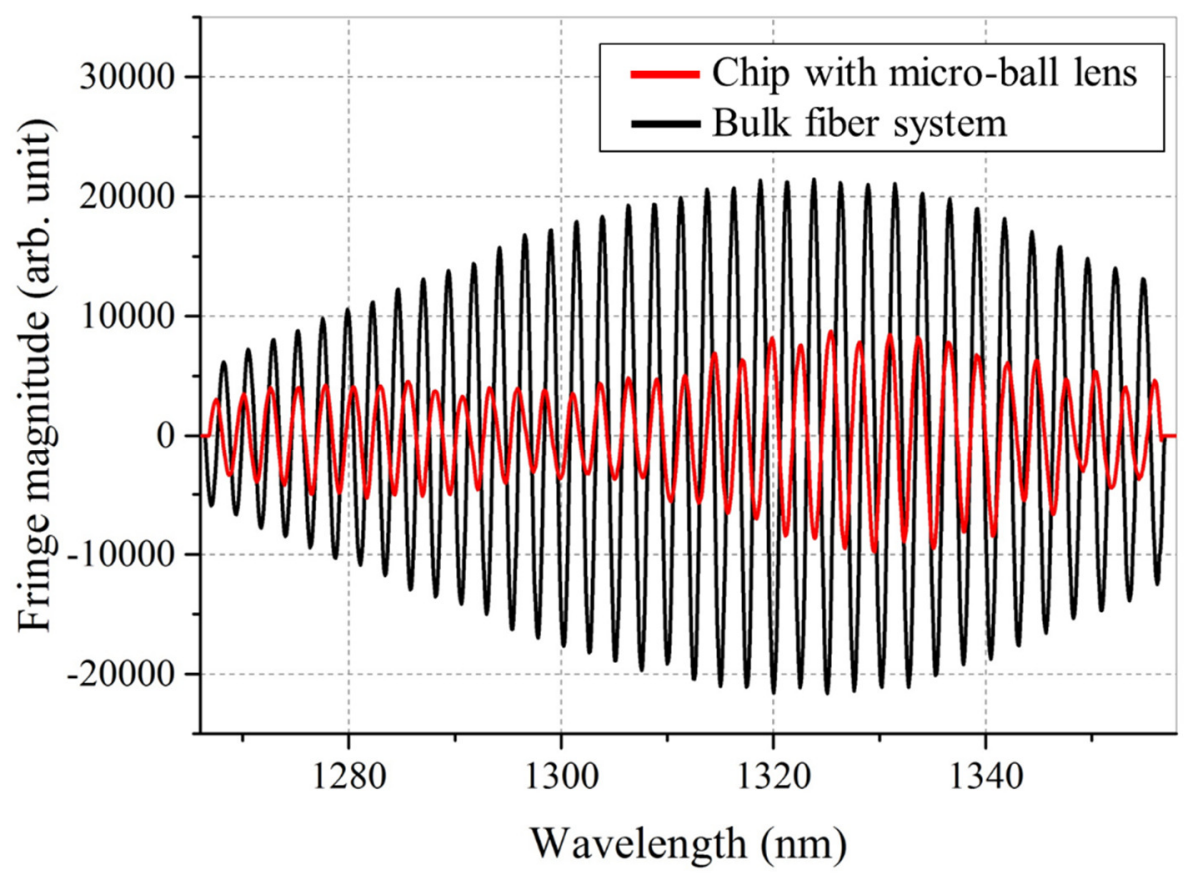

Fig. 5.5.1. Interference fringes [average over 30 spectra and local mean removed in the same way as in Fig 5.4.1(b)] of a reflection from a mirror at $330 \pm 10 \mu \mathrm{m}$ away from the reference plane. The red curve is the measurement result with a chip having an integrated micro-ball lens. The black curve is the measurement result with the bulk fiber system.

Table 5.5.1. Comparison of measured A-scan parameters between the chip system and a fiber system with a mirror sample at $0.3 \mathrm{~mm}$.

\begin{tabular}{|c|c|c|c|c|}
\hline & $\begin{array}{c}\text { Signal peak magnitude } \\
q_{\text {peak }}(\text { arb. units })\end{array}$ & $\begin{array}{c}\text { FWHM } \\
(\mu \mathrm{m})\end{array}$ & $\begin{array}{c}\text { Noise } \sigma \\
(\text { arb. units })\end{array}$ & $\begin{array}{c}\text { SNR } \\
(\mathrm{dB})\end{array}$ \\
\hline $\begin{array}{c}\text { Chip with micro-ball } \\
\text { lens }\end{array}$ & $(5.2 \pm 0.1) \times 10^{3}$ & $11.8 \pm 0.2$ & $1.5 \pm 0.1$ & $71 \pm 1$ \\
\hline Fiber system & $(15.8 \pm 0.1) \times 10^{3}$ & $12.5 \pm 0.2$ & $1.2 \pm 0.1$ & $82 \pm 1$ \\
\hline
\end{tabular}


Therefore, the total transmittance of the chip system from the sample to the detector is $11 \pm 2 \%$, which is not around $6 \%$ as calculated with Equation (2.5.9). This mismatch is due to the fact that the reflected reference power, $14.5 \mu \mathrm{W}$, in case of the chip system is the sum of the contributions from three references as will be discussed in Section 5.6. The reference power that contributes to the main signal peak is only $11.5 \pm 1 \mu \mathrm{W}$ rather than $14.5 \pm 1 \mu \mathrm{W}$, which explains the mismatch within the experiment error.

The power transmittance in the chip system can be improved in the following ways.

1. An anti-reflection coating on the lens could reduce the Fresnel reflection.

2. The sample-chip coupling can be improved by using a larger diameter micro-ball lens which can provide a beam with a Rayleigh range covering the entire range of interest.

3. Using shorter waveguides (from currently $4 \mathrm{~cm}$ to a few $100 \mu \mathrm{m}$ ) to connect the ports of the $\mathrm{Y}$ junction will lead to smaller propagation losses.

4. Using an on-chip low-loss circulator instead of the $\mathrm{Y}$ junction will eliminate its intrinsic 50\% loss. However, the design and fabrication of such a circulator is rather challenging, especially for a broad wavelength range [11].

5. The fiber-chip coupling could be improved by designing a suitable waveguide taper.

Despite the larger signal peak, resulting in an expected larger shot noise, the measured noise is slightly smaller for the fiber system (see Table 5.5.1). The reason is that the fiber system uses a separate reference path, enabling the application of a balanced detector. This significantly reduces the RIN arising from the laser power fluctuation [12]. Thus, the total noise in the fiber system is smaller than in the chip system. One possible solution to reduce the RIN noise in the chip system is to monitor the laser power and apply post processing by software.

The conclusion of this section is that the current chip system with an integrated microball lens has a slightly lower SNR than the fiber system, mainly due to additional losses. Several suggestions have been given accordingly for reducing the losses in the chip system that should improve its performance to the same level as the fiber system.

\subsection{Phantom imaging}

In Section 5.6.1, we first present a recorded OCT image of an optical phantom sample measured with the chip system with a micro-ball lens. We then discussed artifacts that appear in the measured OCT image. In Section 5.6.2, we show how the image quality is improved by reducing the effect of multiple reference planes (one of the artifacts) with the method discussed in Section 2.3. 


\subsubsection{Phantom imaging result and its artifacts}

The setup used to obtain the OCT images of a phantom is the same as the one shown in Fig. 5.3.1, where the mirror sample is replaced by a layered phantom on a translation stage. The translation stage is used to move the phantom laterally with respect to the chip to obtain B-scans. A schematic of the phantom and part of the chip is shown in Fig. 5.6.1. The phantom consists of 3 scattering layers which are separated by 2 transparent layers. The scattering layer, used to mimic the skin, is silicone elastomer-based which includes scattering by adding $\mathrm{TiO}_{2}$ or $\mathrm{SiO}_{2}$ particles [13]. This layered sample is placed on a glass slide. The surface of the phantom is tilted slightly to avoid a direct surface reflection. The back scattered light is collected by the lens and coupled into the waveguide.

A B-scan of the phantom is shown in Fig. 5.6.1, in which 30 spectra is averaged to obtain one A-scan in the B-scan. Three scattering layers are clearly visible in this B-scan, as well as the scattering from the rear side of the glass holder as indicated with red arrows.

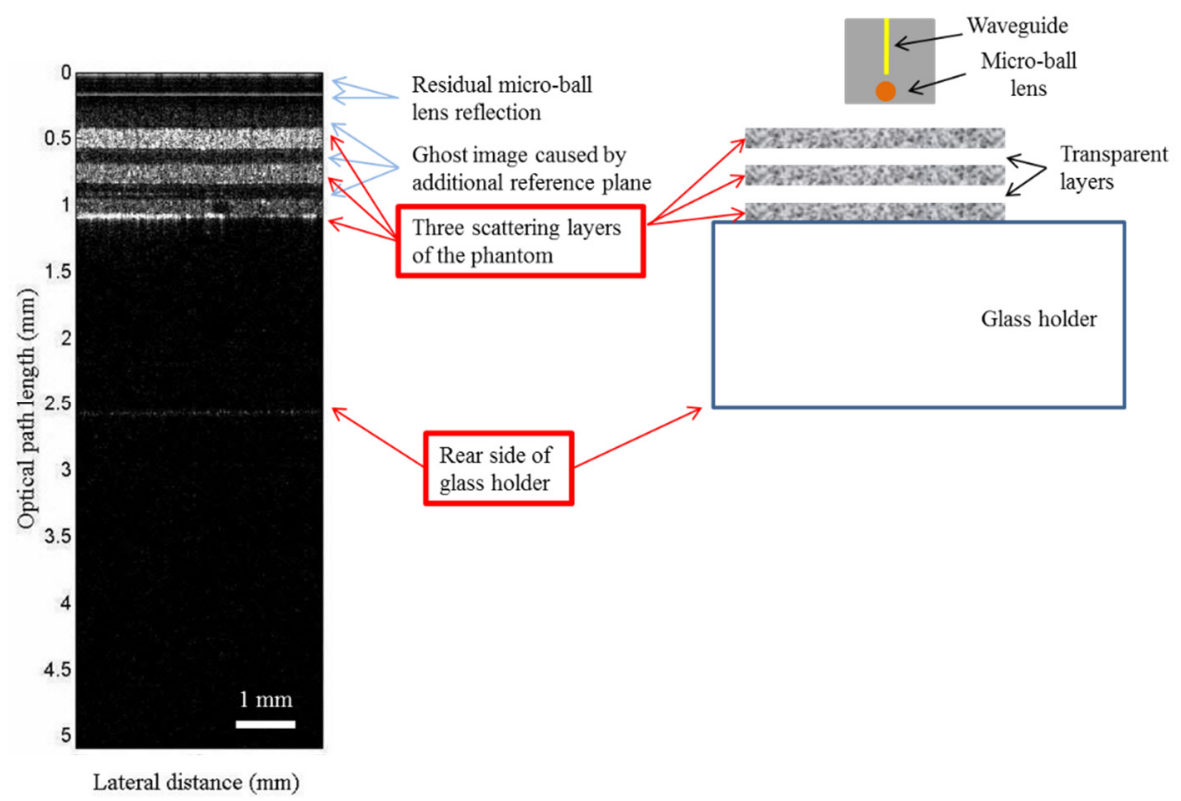

Fig. 5.6.1. The phantom structure and its B-scan result. The right-hand part of the figure is a schematic of the phantom and part of the chip (not to scale). The phantom consists of 3 scattering layers which are separated by 2 transparent layers. This layered sample is placed on a glass slide. The left-hand part of the figure is a B-scan of the phantom. 
Two kinds of artifacts can be noticed (indicated with blue arrows):

1. Two lines close to the zero optical path length.

2. Weak scattering-like image at the position of the two transparent layers and the air layer (above the first scattering layer).

The two lines close to the zero optical path length are caused by the reflections from the micro-ball lens surface. A background measurement without the sample, has been subtracted from the sample measurement to remove these reflection lines. However, the coupling efficiency between the fiber and chip varies a bit from measurement to measurement, which leads to a small power difference in these reflections. This small power difference results in non-complete cancellation of these two lines. This problem is not very severe because these artifacts are always at the same location and are distinct from the sample image. It can be solved by gluing the FAU to the chip, which will completely remove the coupling uncertainty between measurements.

The second type of artifact is potentially much more detrimental to image formation as it consists of ghost images (overlapping the sample image) which are formed by additional reference reflections in the chip system. The source light experiences Fresnel reflections not only at the end facet of the waveguide (the intended reference plane) but also from the front and back surfaces of the micro-ball lens. These lens surfaces act therefore as two additional reference planes in this common-path OCT system. A mathematical model of a multiplereference OCT system is described in Section 2.3 and the main result is Equation (2.3.18), which is given here in a simplified form,

$$
i_{D, \text { image }}(z)=G \otimes H_{P S F},
$$

where $\otimes$ denotes convolution, $G$ and $H_{P S F}$ are functions of wavenumber, $G$ contains the sample information, and $H_{P S F}$ is the point spread function (PSF) [14] of the OCT system in the axial direction, which is independent of the sample. The PSF is composed of the broadening function depending on the source bandwidth, and a function describing the dependence on the reflectivities and relative positions of the reference planes. This PSF can be characterized by measuring the response of a well-known sample (a mirror) as indicated in the Section 2.3. In case of a multiple references OCT like in our chip system, this PSF is a multi-peak function resulting in ghost images for every additional reference plane that may overlap with the image that originates from the primary reference plane. A commonly used imaging processing technique, deconvolution, can be used to suppress or remove these unwanted ghost images [14]. 


\subsubsection{Multiple reference planes induced ghost images suppression}

Here we investigate the applicability of the deconvolution technique for suppressing the ghost images. To this end, the PSF of our chip system is measured with a mirror sample, since $G$ is a delta function in the case of a mirror sample. A zoomed-in view of an A-scan result of a mirror sample is shown by the black curve in Fig. 5.6.2(a), which is the PSF of our system. The effect of the two additional references is clearly visible as two additional peaks (peak 2 and 3) in Fig. 5.6.2(a). The peaks 1, 2 and 3 in Fig. 5.6.2(a) are the interference of the mirrorwaveguide-facet, mirror-lens-surface (close to the waveguide) and mirror-lens-surface (far from the waveguide), respectively.

Next, this PSF is used in the deconvolution of A-scans from any sample measured with this chip system. Simple deconvolution based on its definition suffers from the measurement noise, which lead to non-usable results. Thus a more advanced algorithm, one of the Matlab deconvolution functions 'deconvlucy' (based on the Richardson-Lucy deconvolution [13, 15], which is a commonly used iterative algorithm in imaging processing [16]), is used on the black curve in Fig. 5.6.2(a). The deconvolution result is shown with the red curve in Fig. 5.6.2(a) which shows a strong suppression of the peaks caused by reflections from the lens' surfaces. The FWHM of the main peak after deconvolution is smaller than the one before deconvolution, which indicates a sharpening effect of the OCT image. The amount of suppression of the unwanted peak and sharpening of the main peak depend on the number of iterations with this particular deconvolution algorithm. The example shown in Fig. 5.6.2(a) has an iteration number of 6 . The more iterations used the more suppression on the peaks 2 and 3 in Fig. 5.6.2(a) as well as the noise. However, too many iterations results in the loss of some relatively weak signal (such as weak scattering points in case of an optical phantom), which is processed as noise in this particular deconvolution algorithm.

In theory, the measurement of the $H_{P S F}$ is not sensitive to the position of the mirror sample with respect to the reference planes. Several $H_{P S F}$ measurement using different mirror distances ( $1 \mathrm{~mm}, 2 \mathrm{~mm}, 3 \mathrm{~mm}$, and $4 \mathrm{~mm}$ ) have been performed and shown in Fig. 5.6.2(b). The deconvolved solutions using these $H_{P S F}$ are shown in Fig. 5.6.2(c) and no significant differences are observed in the results.

Some minor differences are noticeable in different $H_{P S F}$, such as the main peak width and the relative magnitude of the three peaks. The deconvolved solutions also shown little difference in the amount of suppression on the lens surface induced peaks. The origin of these minor differences are unknown at this moment, and requires further studies. 

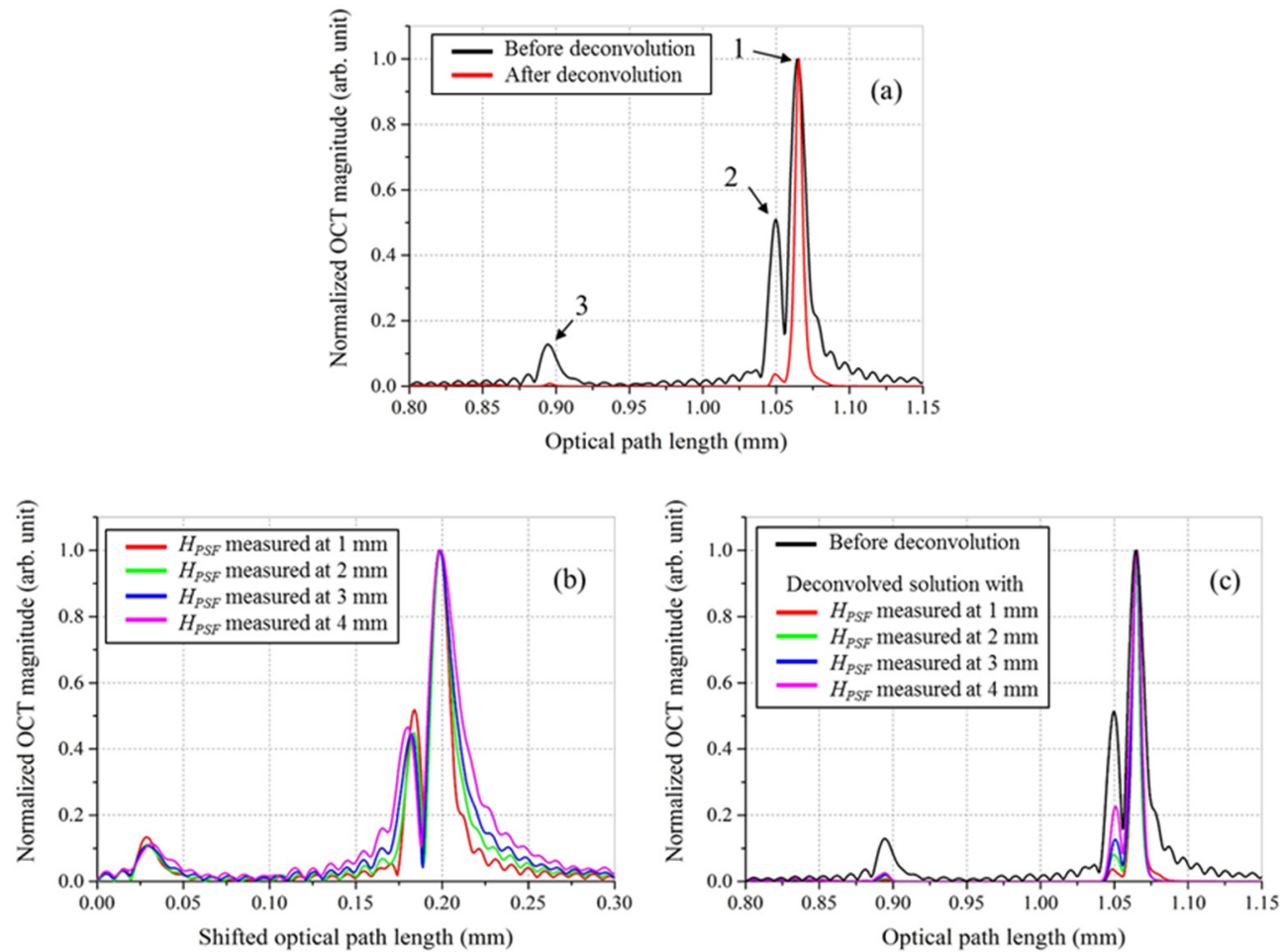

Fig. 5.6.2. (a) A zoomed-in view of an A-scan result of a mirror sample. This plot shows the effect of multiple references on a mirror sample (black curve) and its deconvolved solution (red curve). (b) $H_{P S F}$ measured with the mirror sample at different distance to the waveguide facet. All $H_{P S F}$ have been normalized and shifted to 0 optical path length for proper compression. (c) Deconvolved solutions (colored curves) of an A-scan (black curve) with corresponding $H_{P S F}$ shown in (b).

Finally, we apply the deconvolution (use the $H_{P S F}$ measured at $1 \mathrm{~mm}$ ) to the B-scans of the optical phantom. Two zoomed-in cross-sectional imagines (B-scan) are shown in Fig. 5.6.3. Each A-scan in the B-scan is obtain by averaging over 30 spectra. The image after deconvolution shows a clear improvement in the contrast. This is due to the suppression of the ghost images of the scattering layers.

Next, we measured B-scans of the same optical phantom placed at different distances from the chip. The full range (up to $z_{M A X}$ ) of deconvolved B-scans are shown in Fig. 5.6.4. The image of the phantom becomes dimmer with increasing optical path length. Scattering layers are still clearly visible at an optical path length of $2.6 \mathrm{~mm}$. Scattering (the glass holder and the sample were placed under an angle to avoid specular reflection) from the rear side of the glass holder is even visible up to $4.2 \mathrm{~mm}$. 

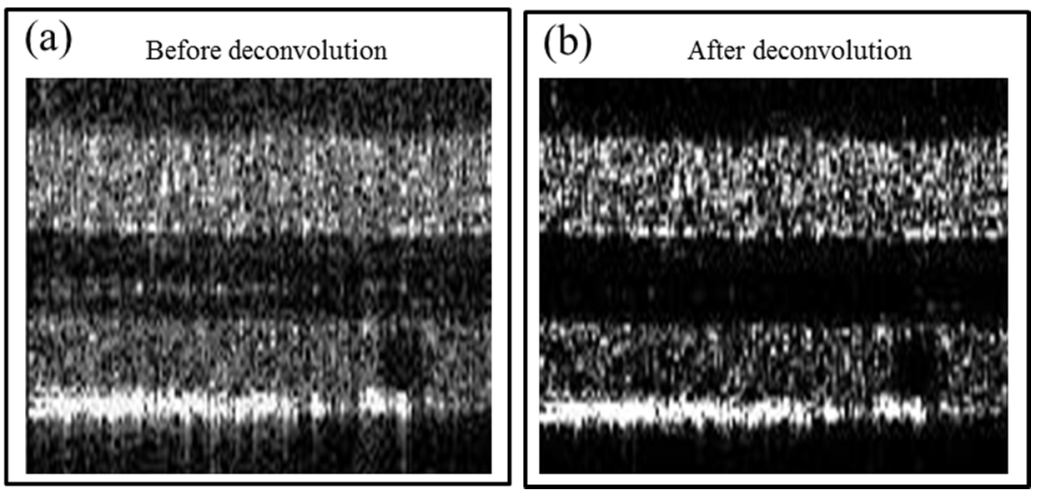

Fig. 5.6.3. Zoomed in cross-sectional images of a phantom sample before (a) and after (b) deconvolution.
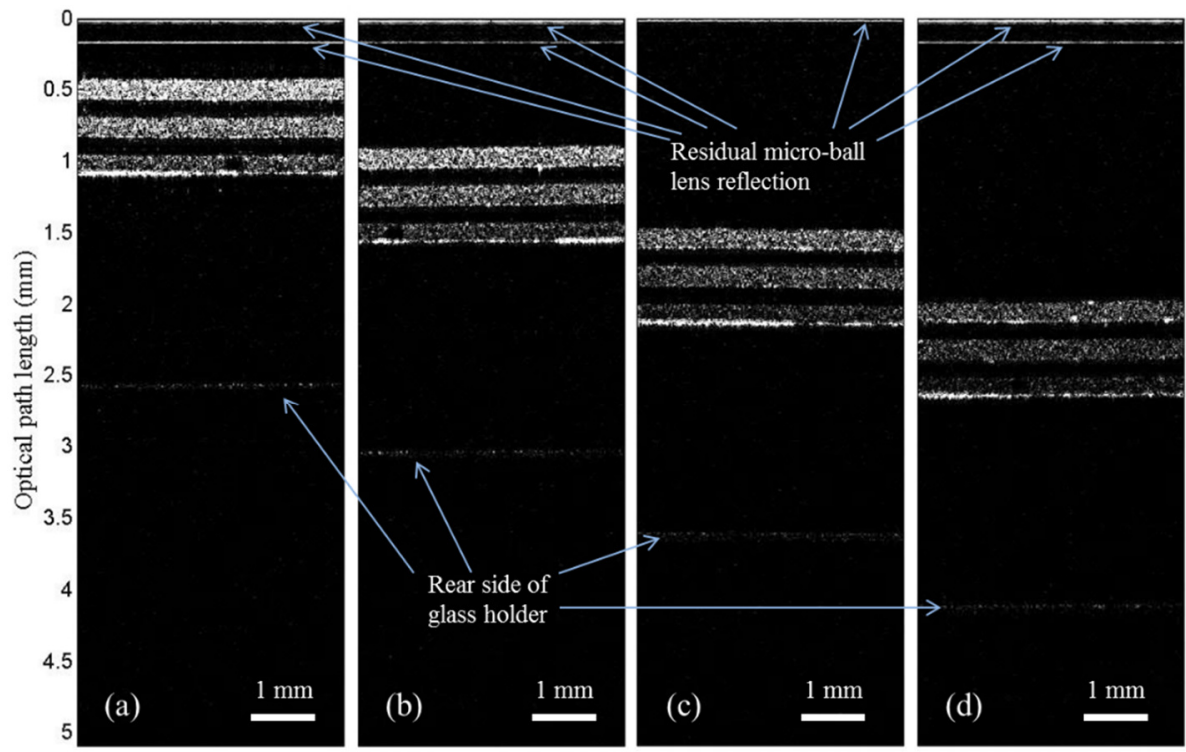

Lateral distance $(\mathrm{mm})$

Fig. 5.6.4. Deconvolved phantom images at different distances from the chip. The distances between the chip edge and the top surface of the phantom are $0.2 \mathrm{~mm}, 0.7 \mathrm{~mm}, 1.2 \mathrm{~mm}$ and $1.7 \mathrm{~mm}$ in (a), (b), (c) and (d), respectively.

Many different deconvolution algorithms exist for image processing. The deconvolution result may be different depending on the noise in the measurement and the algorithm used [17]. Since the described experiment is intended as a proof of concept that the artefacts arising from the multiple reference planes can be suppressed with a deconvolution approach, we did not perform a systematic study of different deconvolution algorithms. A further study on the selection of the deconvolution algorithms may be needed to find the optimal algorithm for this particular system. 


\subsection{Conclusions and outlooks}

In this chapter, we have demonstrated a chip-based common-path swept-source OCT system with an integrated micro-ball lens. The common-path design eliminates the space-consuming and dispersive on-chip loop reference arm. Therefore, no dispersion compensation is needed to achieve the light-source-limited axial resolution. The three-port configuration (light source, common-path arm, and detector) enables the use of a wavelength-independent 50/50 Y junction which is much less sensitive to fabrication errors compared to a directional coupler. The drawback of this common-path configuration is that it cannot use a balanced detector, which leads to around 3-4 dB lower sensitivity compared to a traditional dual-arm OCT [18]. The use of an on-chip micro-ball lens eliminates the need for external optical elements for coupling the light between the chip and the sample. Such a micro-ball lens enables a very short distance of a few hundred $\mu \mathrm{m}$ between the sample and the reference plane, which is an important requirement for common-path OCT. The use of this micro-ball lens leads to a signal enhancement up to $37 \mathrm{~dB}$ compared to the chip without a lens, for a mirror sample. Multiple ghost images caused by additional reference planes (originating from the lens surface) could be largely suppressed using a deconvolution scheme. Finally, cross-sectional imaging of a layered optical phantom, with suppressed ghost image, has been demonstrated.

Since our chip has been designed to be wavelength insensitive in a much larger wavelength range than the swept laser can offer, we are planning to use a SD-OCT configuration, in the future, to study the performance of the chip in a wavelength range of 1.2-1.7 $\mu \mathrm{m}$.

The minimum footprint of this design could, in principle, be as small as $\sim 400 \times 100 \mu \mathrm{m}^{2}$, which may enable the fabrication of a chip-based parallel OCT system with multiple independent detection channels. The minimum length of $\sim 400 \mu \mathrm{m}$ is determined from the $200-\mu \mathrm{m}$ long $\mathrm{Y}$ junction plus the $100-\mu \mathrm{m}$ diameter micro-ball lens, plus some spacing. (However, in order to facilitate testing of the device, our prototype was equipped with several $\mathrm{cm}$ long straight access waveguides.) The minimum width of $\sim 100 \mu \mathrm{m}$ is determined by the diameter of the lens.

The B-scans demonstrated here were obtained by translating the sample. Since the micro-ball lens is integrated and the optical chip is a small low-mass device, an alternative implementation might mount the fiber-connected chip directly on a scanner to obtain two- or three-dimensional images.

We believe that, by integrating a micro-ball lens onto the chip and using a common-path configuration, we have moved a significant step forward in the development of on-chip SS-OCT systems. 


\section{References}

1. Huang, W.-P. and C. Xu, Simulation of three-dimensional optical waveguides by a full-vector beam propagation method. Quantum Electronics, IEEE Journal of, 1993. 29(10): p. 2639-2649.

2. Yun, S., G. Tearney, B. Bouma, B. Park, and J. de Boer, High-speed spectral-domain optical coherence tomography at $1.3 \mu \mathrm{m}$ wavelength. Optics Express, 2003. 11(26): p. 3598-3604.

3. Wickramarachi, P., Effects of windowing on the spectral content of a signal. Sound and Vibration, 2003. 37(1): p. 10-11.

4. Goldberg, B.D., B.J. Vakoc, W.Y. Oh, M.J. Suter, S. Waxman, M.I. Freilich, B.E. Bouma, and G.J. Tearney, Performance of reduced bit-depth acquisition for optical frequency domain imaging. Optics Express, 2009. 17(19): p. 16957-16968.

5. Dufour, M., G. Lamouche, B. Gauthier, C. Padioleau, and J.-P. Monchalin. Inspection of hard-to-reach industrial parts using small-diameter probes. in Proceedings of the SPIE. 2006.

6. Song, G. and K. Harding. OCT for industrial applications. 2012.

7. Walecki, W.J., K. Lai, A. Pravdivtsev, V. Souchkov, P. Van, T. Azfar, T. Wong, S.H. Lau, and A. Koo. Low-coherence interferometric absolute distance gauge for study of MEMS structures. 2005.

8. Walecki, W.J., K. Lai, V. Souchkov, P. Van, S.H. Lau, and A. Koo, Novel noncontact thickness metrology for backend manufacturing of wide bandgap light emitting devices. E-MRS 2004 Fall Meeting Symposia C and F, 2005. 2(3): p. 984-989.

9. Weiss, N., T.G. van Leeuwen, and J. Kalkman, Localized measurement of longitudinal and transverse flow velocities in colloidal suspensions using optical coherence tomography. Physical Review E, 2013. 88(4): p. 042312.

10. Akca, B.I., Spectral-domain optical coherence tomography on a silicon chip. 2012.

11. Pintus, P., F. Di Pasquale, and J.E. Bowers, Integrated TE and TM optical circulators on ultra-low-loss silicon nitride platform. Optics Express, 2013. 21(4): p. 5041-5052.

12. Chen, Y.L., D.M. de Bruin, C. Kerbage, and J.F. de Boer, Spectrally balanced detection for optical frequency domain imaging. Optics Express, 2007. 15(25): p. 16390-16399.

13. Richardson, W.H., Bayesian-based iterative method of image restoration. JOSA, 1972. 62(1): p. 55-59.

14. Shaw, P.J. and D.J. Rawlins, The Point-Spread Function of a Confocal Microscope - Its Measurement and Use in Deconvolution of 3-D Data. Journal of Microscopy-Oxford, 1991. 163: p. 151-165.

15. Lucy, L.B., An iterative technique for the rectification of observed distributions. The astronomical journal, 1974. 79: p. 745.

16. Hojjatoleslami, S.A., M.R.N. Avanaki, and A.G. Podoleanu, Image quality improvement in optical coherence tomography using Lucy-Richardson deconvolution algorithm. Applied Optics, 2013. 52(23): p. 5663-5670.

17. Starck, J.L., E. Pantin, and F. Murtagh, Deconvolution in astronomy: A review. Publications of the Astronomical Society of the Pacific, 2002. 114(800): p. 1051-1069.

18. Zhao, M., Y. Huang, and J.U. Kang, Sapphire ball lens-based fiber probe for common-path optical coherence tomography and its applications in corneal and retinal imaging. Optics Letters, 2012. 37(23): p. 4835-4837. 


\section{Chapter 6}

\section{Parallel OCT on Chip}

\subsection{Introduction of full-field OCT (FF-OCT) and parallel OCT}

\section{(P-OCT)}

In a conventional OCT, B-scans and C-scans are obtained by mechanically scanning a focused beam over the sample as introduced in Section 1.2. The mechanical scanning speed is one of the limiting factors for the OCT imaging speed. With improved technology, nowadays the fundamental speed limit is determined by the maximum allowed photon flux (the number of photons per unit area and unit time) on the tissue [1]. In a shot noise limited FD-OCT system the SNR is given by Equation (2.5.12), reproduced here below:

$$
S N R=\frac{\rho M \tau_{i}}{e} \frac{P_{R} P_{S}}{P_{R}+P_{S}},
$$

where $e$ is the charge of an electron, $\rho$ is the average responsivity of the detector, $M$ is the total number of spectral channels, $\tau_{i}$ is the integration time of each spectral channel and $P_{R}$, $P_{S}$ are the average powers at the detector of all spectral channels due to reflection from respectively the reference and the sample. With a given lateral resolution (focused beam size) the maximum allowed photon flux implies maximum values for the power quantities $P_{R}$ and $P_{S}$ in Equation (6.1.1). Thus, for given powers an increased imaging speed (smaller $\tau_{i}$ ) will result in reduced SNR, and therefore in lower image quality. Increasing the imaging speed without sacrificing the SNR can only be attained by parallel image acquisition in the lateral direction. For example, in case of retinal imaging, $\sim 67$ times larger total power is allowed in a parallel illumination system compared with a single focused scanning illumination [1]. This increased power can be exploited for attaining correspondingly faster imaging. 
(a)

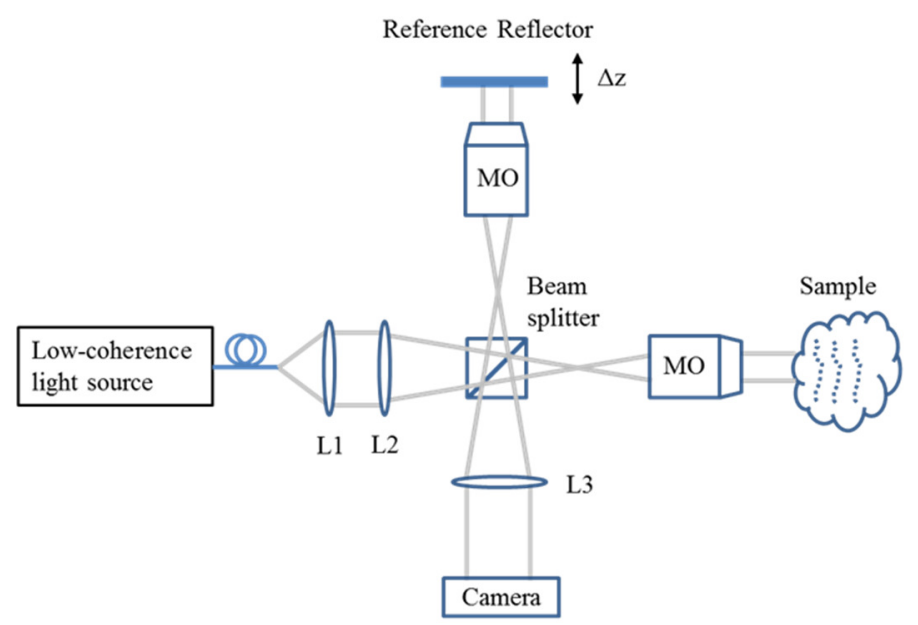

(b)

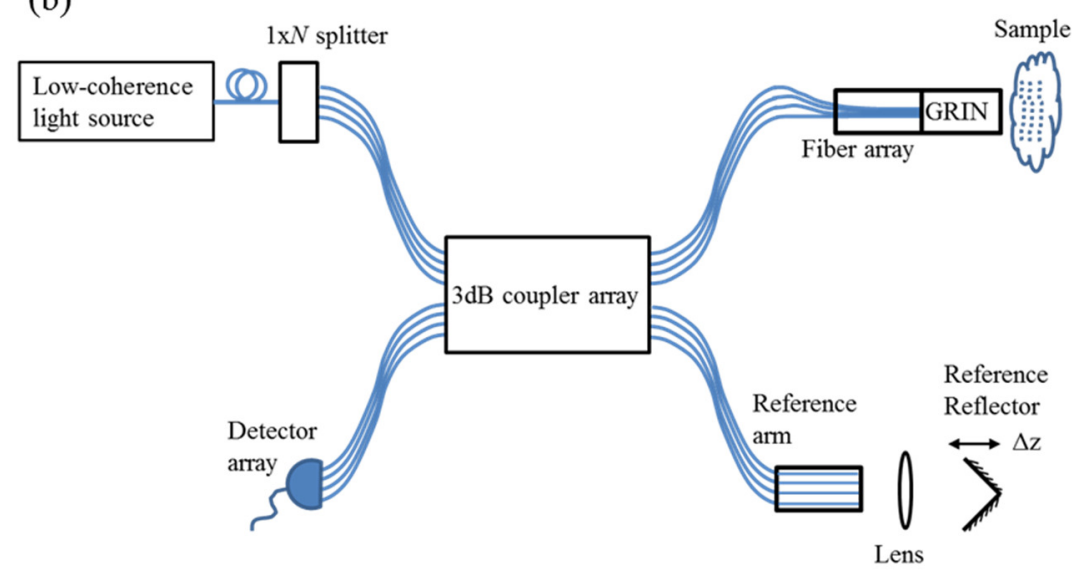

Fig. 6.1.1. Schematic of time domain FF-OCT (a) and P-OCT (b). MO: microscope objective. L1, L2 and L3: lenses. 3dB coupler array: fiber based 50/50 splitters. GRIN: gradient-index lens.

Currently, there are two types of OCT systems which are designed for parallel illumination and image acquisition in the lateral direction, namely, full-field OCT (FF-OCT) and parallel OCT (P-OCT). FF-OCT is free-space-interferometer-based and normally built with a microscope configuration [1-3]. Therefore, some studies refer to this type as full-field optical coherence microscopy [4]. A schematic of a time domain FF-OCT is shown in Fig. 6.1.1(a). In this FF-OCT, the entire field (of the OCT image) of both sample and reference reflectors are illuminated simultaneously through lenses L1, L2 and two microscope objectives (MO). Lens L3 is used to image both sample and reference planes on to a charge-coupled device (CCD) or a complementary metal-oxide-semiconductor (CMOS) camera. The interference signals at all the lateral locations are then measured simultaneously by the detector array $[1,2,4,5]$. 
Current P-OCTs are free-space-based [6] or fiber-based systems [7, 8], which have a number of $(N)$ lateral illumination and imaging locations, implemented as individual OCT detection channels. (It should be noted however that some authors refer to FF-OCT as P-OCT, such as in [3].) Fiber-based time domain P-OCT systems have been developed for endoscopy applications [8]. A schematic of this P-OCT is shown in Fig. 6.1.1 (b). The light source power is split into 8 individual TD-OCTs where the sample arm consists of an assembled 8-fiber array and a gradient-index (GRIN) lens. The fiber spacing in the fiber array (15 $\mu \mathrm{m}$ center to center spacing) together with the GRIN lens determines the lateral resolution.

These FF-OCTs and P-OCTs are much more bulky and costly than a single channel OCT. Therefore the development of chip-based P-OCT can lead to considerable size and cost reduction. The concept of the fiber-based P-OCT can be conveniently adapted to a chip-based system, where parallelization is realized by duplicating the single channel device and placing them closely. Thus, in this chapter, we present a design of a chip-based parallel SS-OCT (PSS-OCT) and its fabrication result. Due to time limitations, full characterization and demonstration of the PSS-OCT have not yet been performed. We discuss some possible characterization measurements which can be used to study the system performance as a function of relevant design parameters. After that we discuss potential problems and their corresponding solutions in the parallelization of the chip-based SS-OCT system that has been described in the preceding chapters.

\subsection{Chip-based parallel swept-source OCT (PSS-OCT) designs}

The development of chip-based P-OCT is at a very early stage. At this point, our design aim is to demonstrate the feasibility of the chip-based P-OCT without any specific performance target. Thus the design concept is very straightforward. The aim is to fabricate $N$ number of individual OCT detection channels (waveguides and lenses) side by side on the chip. Every detection channel is identical to the OCT chip described in Section 5.3 and the external laser light is split into the $N$ detection channels by an on-chip 1-to- $N$ power splitter.

Our goal is an 8-channel device. For the purpose of illustrating the concept, a 4-channel example is shown in Fig.6.2.1. In this example, 4 single channel SS-OCTs are placed side by side on the same chip. The input light is equally split by a 1 to 4 splitter into all these individual SS-OCTs, and the output signal from all 4 channels are coupled to 4 detectors through a fiber array. The 1 to 4 power splitter is built with cascaded Y junctions. 


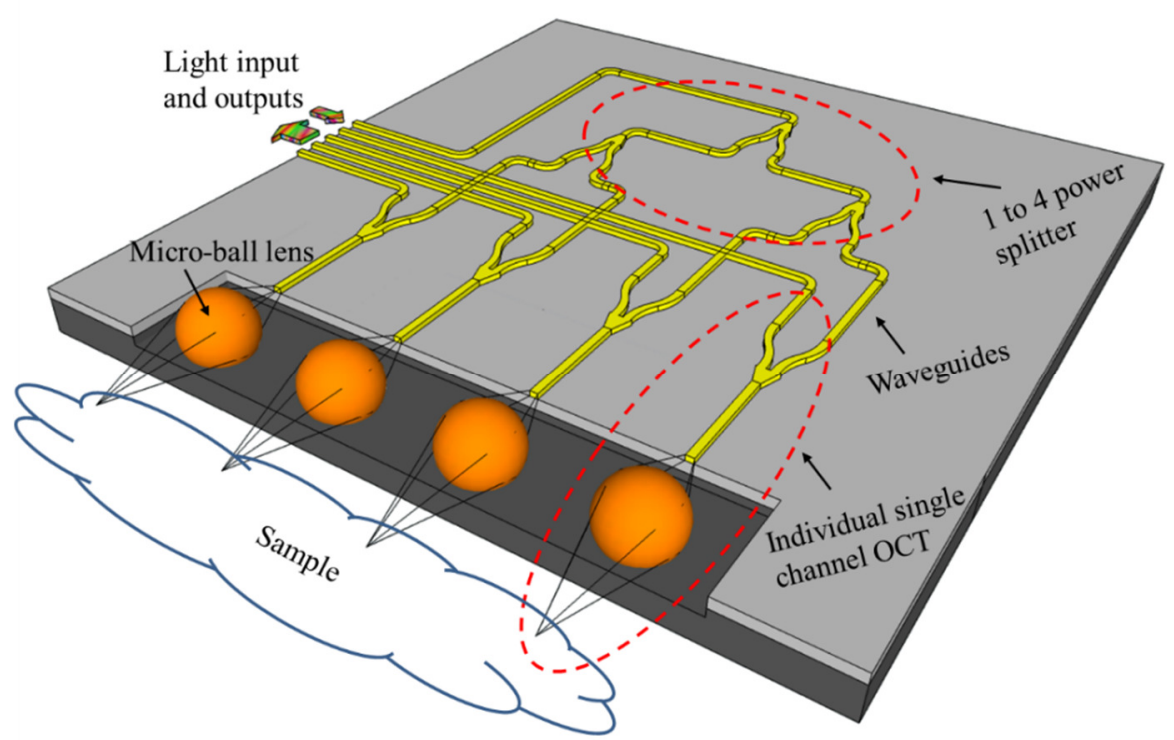

Fig. 6.2.1. Schematic of the PSS-OCT chip design concept for a 4-channel device. In order to better show the waveguides, the top cladding layer $\left(\mathrm{SiO}_{2}\right)$ is not drawn in this figure.

The realized device is an 8-channel PSS-OCT and its layout is shown in Fig. 6.2.2. This design is not aiming for minimum chip size but is specially made for testing the crosstalk between channels (light coming from one channel and detected by another channel, see crosstalk discussion Section 6.3.2). We plan to measure the crosstalk as a function of the channel spacing. Therefore, a non-uniform channel spacing is designed, which can be seen in the upper right corner of the chip in Fig. 6.2.2. The spacing between adjacent channels is designed to be $100+n \times 50 \mu \mathrm{m}$, where $n$ is $0,1,2, \cdots 6$. The minimum spacing of $100 \mu \mathrm{m}$ is approximately the diameter of the micro-ball lens.

The non-uniform channel spacing design itself is not enough to allow the measurement of the crosstalk between channels. In a fiber based P-OCT, the crosstalk can be measured by illuminating only one channel and measuring the response at the other channels [7]. Unfortunately, this test is not possible with our chip-based PSS-OCT since all channels are illuminated simultaneously. This causes the optical power in a channel due to crosstalk from other channels to be mixed with the reflected power in the channel itself. However, by designing the individual OCTs with different total waveguide length, the crosstalk is expected to happen at different optical path lengths compared with the reflection of its own channel (see crosstalk discussion in Section 6.3.2). Therefore, all the individual OCTs have been designed with different optical path lengths and we plan to measure the crosstalk by measuring the OCT signal of the PSS-OCT chip. 


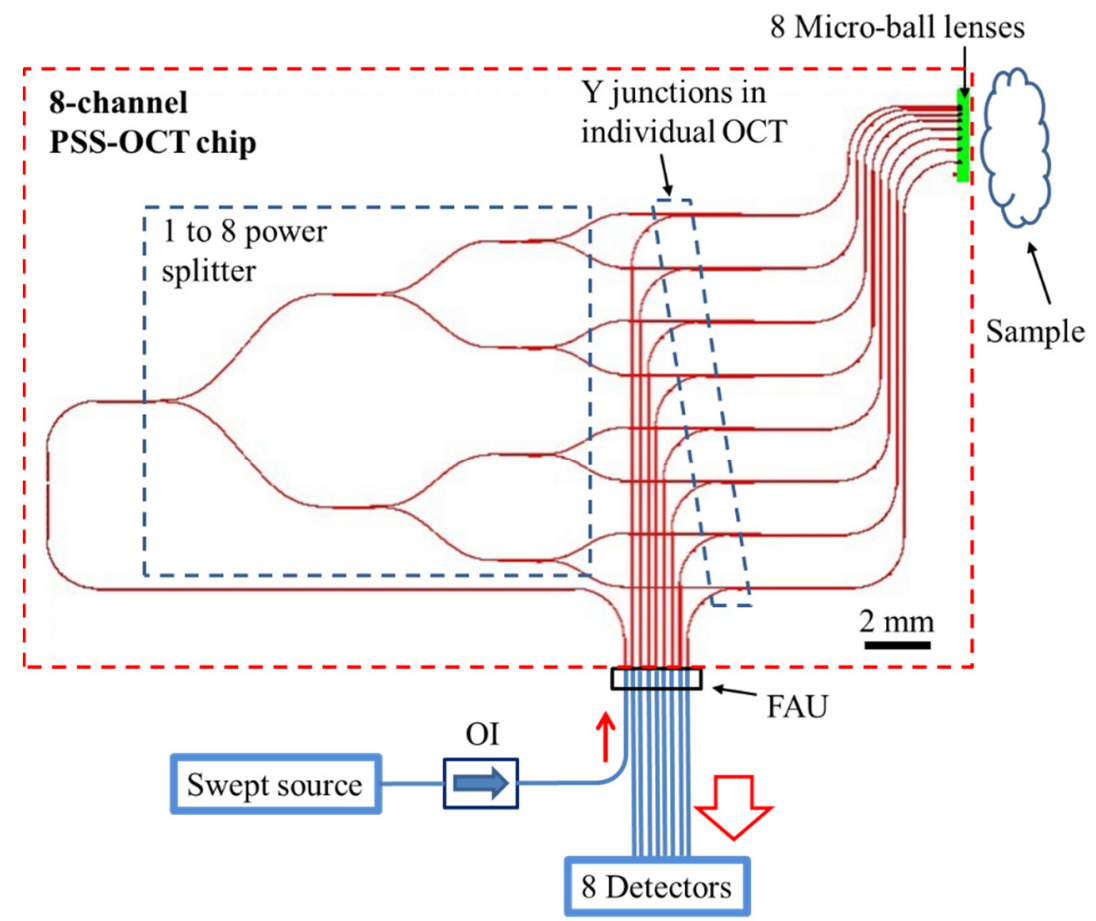

Fig. 6.2.2. System layout of a chip-based 8-channel PSS-OCT. The size of the part inside the red dashed box is proportional to the real chip. The green part in the upper right corner indicates the etched platform for the micro-ball lenses. The 8 lenses are in the green region.

\subsection{Fabrication results}

\subsubsection{Fabricated PSS-OCT chip}

The parallel OCT chip is fabricated in the same way as the single channel OCT as described in Chapter 3 and Chapter 4. The result is shown in Fig. 6.3.1 and Fig. 6.3.2. The minimum gap between the lenses that has been realized is in the order of $1 \mu \mathrm{m}$ as can be seen from Fig. 6.3.1 (a) and Fig. 6.3.1 (b). Unfortunately, one of the lenses (the second one from the left in Fig. 6.3.1) is lost during dicing as can be seen in Fig. 6.3.1 (c). A method to minimize the risk of losing lenses is discussed in Section 4.3.5. 
(a)

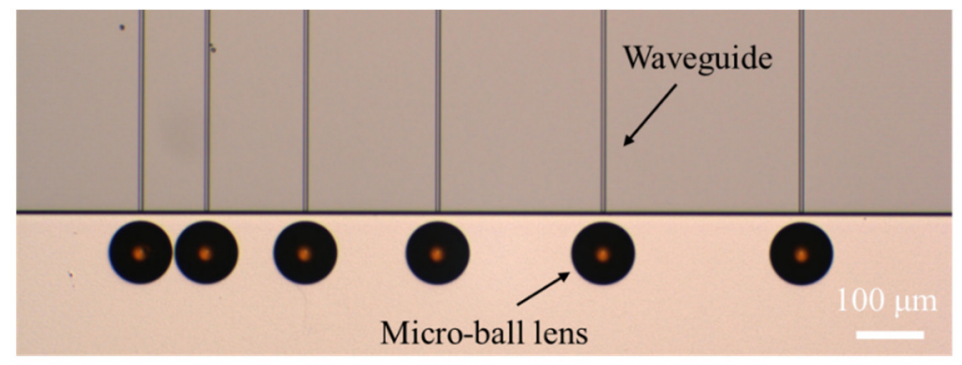

(b)

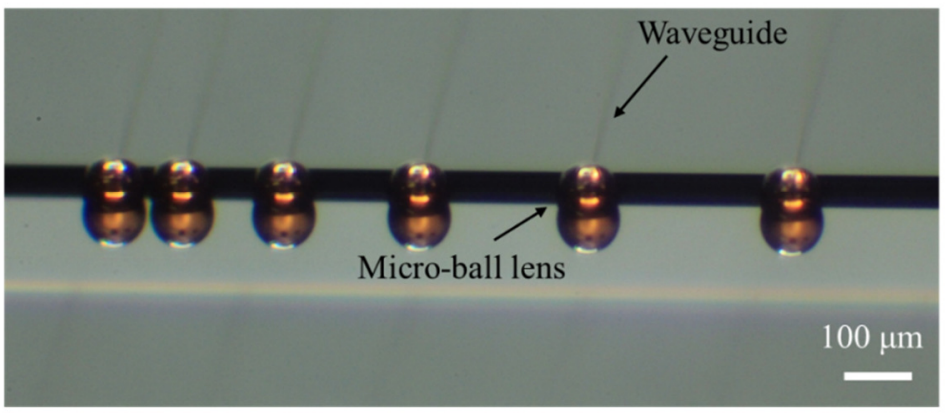

(c)

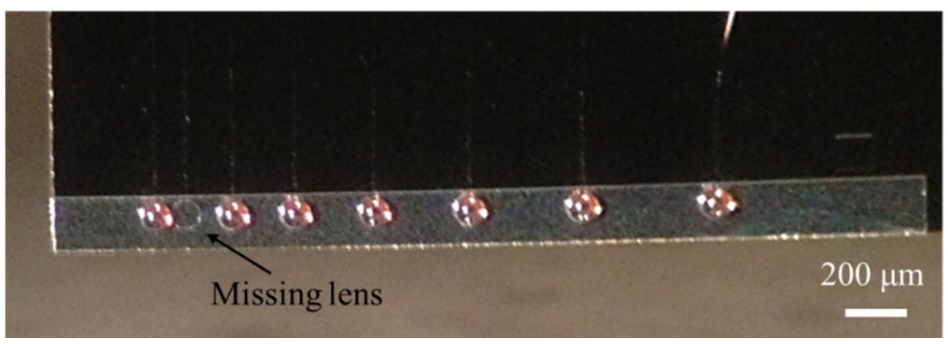

Fig. 6.3.1. Microscopy images of the fabricated lens array of the PSS-OCT chip. (a) Top view of part of the lens array before dicing the chip. (b) Bird's-eye view of same sample before dicing. (c) Top view of the entire lens array after dicing. Note, the second lens from left is missing after dicing. The contact line (a circle) between the missing lens and the surface is still visible.

Part of the lightpath on the PSS-OCT chip is shown in Fig. 6.3.2 (a). Here we used a $630 \mathrm{~nm}$ (wavelength) laser to be able to visualize the light propagation by the waveguide channels. The light which is back reflected from the chip facet and the paper, going to the detectors, is too weak to be seen in this image. An infrared $(\lambda=1310 \mathrm{~nm})$ image in Fig. 6.3.2 (b) gives a direct impression of the sampling locations of the OCT. The detailed beam size is described in Section 4.5. The second OCT channel [count from the top in Fig. 6.3.2 (b)] lost its lens during the dicing. The light exit from this channel spreads so much that its intensity is too weak to be seen in this image. This gives a qualitative impression of the micro-ball lens collimation ability. 


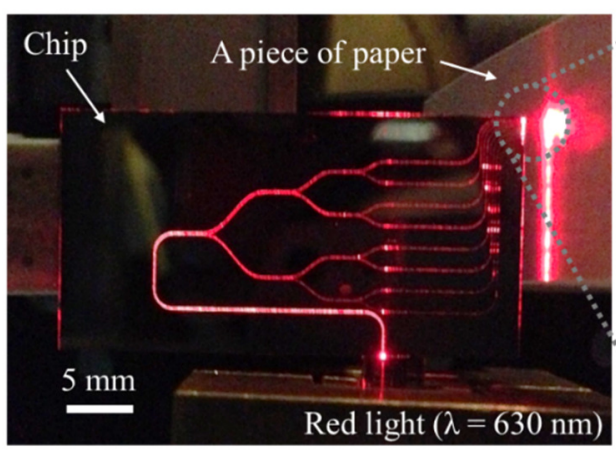

(a)

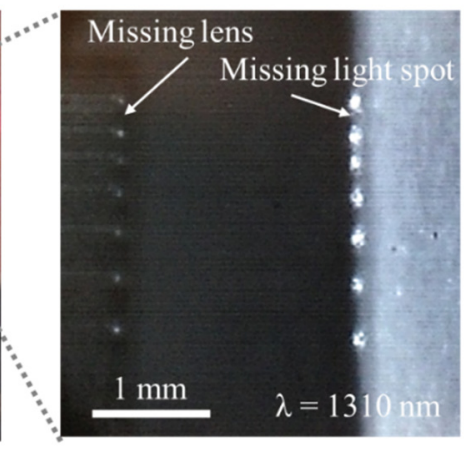

(b)

Fig. 6.3.2. Illustration of the light path in the chip and beam spots on the sample. (a) Red light is coupled into the PSS-OCT chip through a FAU to show the waveguides on the chip (The visible light scatters more than the infrared in the waveguide thus better indicates the lightpath.). It should be noted that this chip is not designed for visible light ( $\lambda=630 \mathrm{~nm}$, multi-mode in this waveguide) thus the red light is split unevenly to different OCT channels. (b) Magnified infrared $(\lambda=1310 \mathrm{~nm})$ image of the lens array and corresponding light spots on a paper. This image is taken by an infrared camera mounted on a microscope. The chip and the paper are illuminated with an incandescent lamp from the side to light them up in the image.

The results show that the fabrication process needs to be further improved to reduce the risk of missing lenses. However, even with one lens missing, the chip is ready for future OCTrelated tests.

\subsubsection{Possible PSS-OCT characterization in the future}

In this section, we suggest experiments to characterize the performance of the designed PSSOCT system.

First, demonstrate the imaging ability of the chip by building the PSS-OCT system and imaging mirror and thin phantom samples. The signal of a given channel due to crosstalk between channels is shifted by an amount of the order of $1 \mathrm{~mm}$ or more in optical path length with respect to the OCT signal from that channel. Therefore, the crosstalk should not overlap with the image of a mirror or a thin (less than $1 \mathrm{~mm}$ optical path length) sample. Imaging of thin $(<1 \mathrm{~mm})$ samples is expected to be possible regardless of the strength of the crosstalk.

Second, investigate the crosstalk as a function of the lens center spacing, sample location and sample type (mirror or scattering sample). The crosstalk between channels reduces the OCT image quality. Therefore, it is important to know which factors influence the crosstalk and how strong their effect is. We expect the crosstalk between OCT channels to be caused by the following effects (the possible crosstalk induced by waveguide crossing is discussed later). 
- Direct light coupling between the adjacent waveguides (OCT channels) through the evanescent field. Although the coupling is expected to be negligible with the large waveguide spacing (100 $\mu \mathrm{m}$ or more) in the current design, this effect may become important if a much higher integration density (smaller waveguide spacing) is used.

- Light exiting from the waveguide of one channel and scattered or reflected from the lens surface to the lens of adjacent channels and then scattered or reflected into the corresponding channels. This effect is expected to be dependent on the channel spacing. (It should be noted that the lens diameter and lens-waveguide distance are fixed and all the same in our current design. Thus, their influences are not considered here.)

- Light reflected or scattered by the sample to the adjacent channel. This effect is expected to be dependent on the channel spacing, sample properties (reflecting or scattering), and sample to chip distance.

All the crosstalk effects mentioned above are expected to be observed at different optical path length differences (in the order of few hundreds of micrometers to few millimeters). Thus, even though there are many possible effects and influencing parameters, the crosstalk can be investigated systematically.

Third, investigate the effects of waveguide crossings on the performance. As can be seen from Fig. 6.2.1 and Fig. 6.2.2, the number of waveguide crossings increases with increasing number of parallel channels. Therefore, the effects introduced by crossings may have significant influence on future systems with more parallel channels. Currently, the effect of crossings on the OCT signal is unknown. However, the loss and crosstalk (the amount of light propagating into the other waveguide at the crossing) of the waveguide crossing itself has been studied [9-11]. The amplitude of the OCT signal is expected to be reduced due to the loss of the crossing. The crossing-induced crosstalk (optical path length difference in the order of few centimeters) may have a similar effect as the OCT channel crosstalk since the light in one channel is coupled into other channels through the crossing. The effect may be more complex since a waveguide from one channel may cross the waveguides from many other channels as shown in Fig. 6.2.2. Therefore, it is wise to first study the crosstalk caused by the OCT channel spacing, sample location and sample type, as mentioned above. Then the remaining crosstalk effects caused by the waveguide crossings can be analyzed.

These suggested experiments have been considered during the design of the chip. Thus, features in the chip are designed to facilitate these experiments. In order to investigate the crosstalk as a function of the lens center spacing and to minimize the influence from other structures on the chip, all the OCT channels have been designed to have the same bending structures and the same number of the waveguide crossings. This led to the current chip layout which does not have the minimum possible number of waveguide crossings. 
With the characterization studies mentioned above, we believe many system performance data can be extracted. This information can be used to further improve the P-OCT chip design in the future.

\subsection{Discussion}

In this section, we discuss some possible improvements to the P-OCT chip design.

\subsubsection{Possible layout to reduce channel spacing}

The minimum channel spacing $D_{c}$ in the current design is limited by the diameter $(\sim 100 \mu \mathrm{m})$ of the lens as shown in Fig. 6.4.1 (a). This is limiting the lateral parallel sampling density of the P-OCT system. An alternative design as shown in Fig. 6.4.1 (b) reduces the minimal channel spacing $D_{c}$ ' to approximately $70 \mu \mathrm{m}$ (half of the lens diameter $\sim 50 \mu \mathrm{m}$ plus half of the beam size just behind the lens $\sim 20 \mu \mathrm{m}$, see Fig. 4.5.2). This improves the lateral sampling density accordingly.

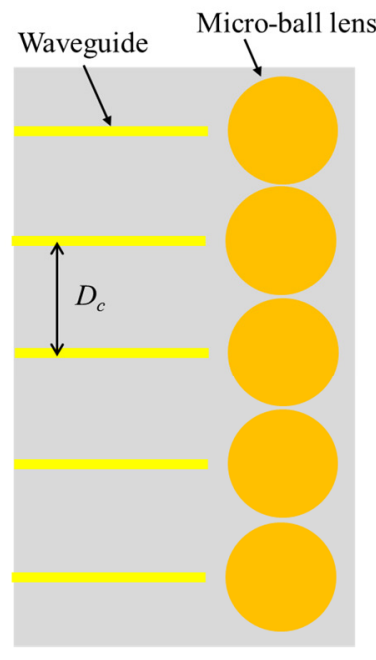

(a)

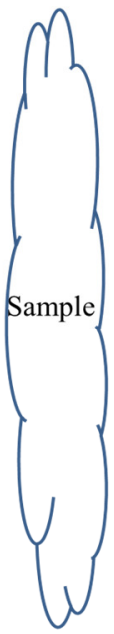

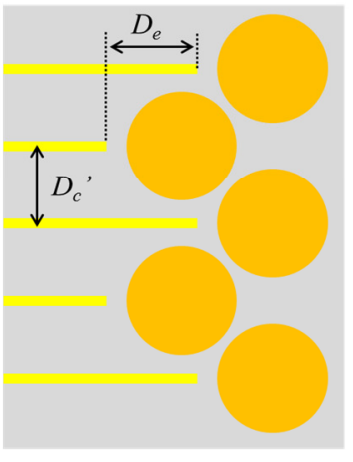

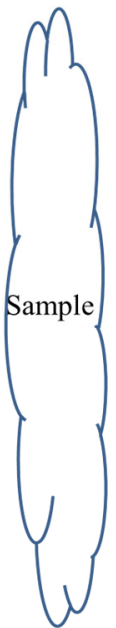

(b)

Fig. 6.4.1. Illustration of the minimum channel spacing. (a) In current design, where all lenses are located on the same line. (b) The suggested layout to reduce the minimum channel spacing by placing the lenses in two lines.

Two aspects need to be taken into account when designing the system in Fig. 6.4.1 (b). First, the crosstalk is expected to be increased due to the reduced channel spacing. Therefore, it is important to know the magnitude of the crosstalk as mentioned in Section 6.3.2. That 
result can be used to decide how far the channel spacing can be reduced before the additional crosstalk becomes unacceptable in view of the overall OCT system imaging requirements. Second, the reference planes (waveguides facets) have different distances to the sample in the adjacent channel. Therefore, an additional software processing step is needed to shift the location of the A-scans in every other channel by $D_{e}$ in the axial direction.

This modification does not need to change the fabrication procedure. Therefore, it can be implemented within a relatively short time.

\subsubsection{Possible solutions to avoid waveguide crossing in P-OCT}

In Section 6.3.2, the possible influence of the waveguide crossing has been discussed. In this section we propose three solutions to eliminate waveguide crossings on a P-OCT chip. All these solutions are discussed based on the design in Fig. 6.2.2.

The first approach is designing the waveguides such that they cross each other at a non-zero vertical distance. In a P-OCT chip similar to the one in Fig. 6.2.2, when the number of parallel channels is equal to or larger than 3 , there are unavoidable waveguide crossings regardless of the design. We propose to build waveguide structures in two layers on top of each other with a few micrometers vertical distance. The two waveguides do not need to cross in the same plane anymore. With a proper design, the crossing-induced loss and crosstalk should be reduced significantly compared to any designs in the single-layer process.

Structures will be needed for controlled coupling of light between the layers. Vertical couplers with $50 \%$ or $100 \%$ coupling ratio may be useful. A 50/50 vertical coupler can be realized as a broad band directional coupler replacing the $\mathrm{Y}$ junction in the single channel OCT, although the wavelength independence of the directional coupler is not as good as that of a Y junction. However, it is very challenging to fabricate a vertical Y junction. Therefore, a Mach-Zehnder-type vertical directional coupler is suggested to be used as the 50/50 vertical coupler. A single layer Mach-Zehnder directional coupler has been proved to have a much smaller wavelength dependence compared to a simple directional coupler [12]. The suggested design in [12] is shown in Fig. 6.4 .2 (a). It consists of a 50/50 directional coupler, a 100\% coupler and a $2 \pi / 3$ phase shift in one of the arms (all these parameters are applied to the center wavelength). Our proposed vertical coupler is shown in Fig. 6.4.2 (b), where the design principle is exactly the same as the single-layer coupler. In order to eliminate the alignment uncertainty between the two layers $( \pm 1 \mu \mathrm{m})$, the two directional couplers are realized with lines having small angles (in the order of few degrees) with respect to each other. This non-parallel design ensures that the misalignment only changes the location of the coupler but not the coupling ratio. It should be noted that the photolithographic angular misalignment $\left(\sim 10^{-3}\right.$ degree $)$ is negligible in a $100 \mathrm{~mm}$ diameter wafer. 
(a)

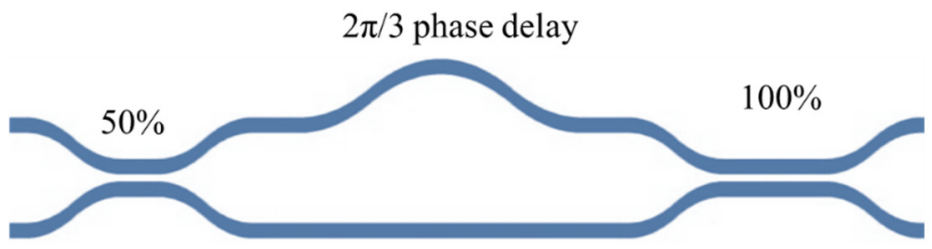

(b)

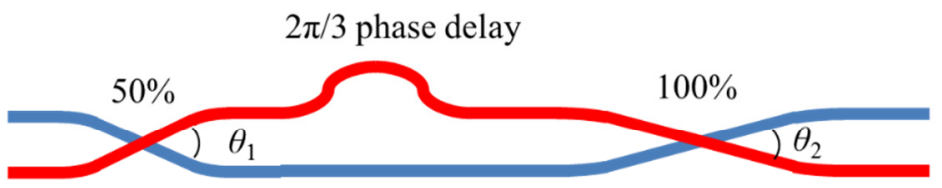

(c)

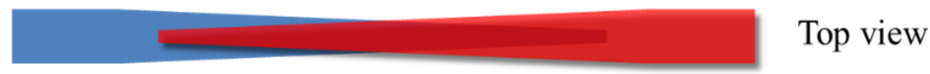

Fig. 6.4.2. Broadband coupler designs. (a) Single layer Mach-Zehnder coupler with an optimal phase delay for broad band 50/50 coupling. (b) Two layer Mach-Zehnder coupler with the same design principle as in (a). The non-parallel $50 \%$ and $100 \%$ coupler design is used to eliminate the misalignment influence. (c) Non-uniform adiabatic $100 \%$ vertical coupler design with two tapers (one in each layer) arranged in opposite direction.

The other idea of the $100 \%$ vertical coupler approach is to bring all the light from one layer to another layer to avoid the crossing in the same layer. For instance, suppose that the fiber array (FAU) is coupled to waveguides in the bottom layer. Then the light from the source should be brought to the top layer using a $100 \%$ vertical coupler in order to cross the detector-coupled channels at a different vertical level. After $1 \times N$ splitting and passing all crossing locations (such as the ones in Fig. 6.2.2), the light is coupled back to every individual OCT channel in the bottom layer. Our proposed non-uniform adiabatic $100 \%$ vertical coupler is shown in Fig. 6.4.2 (c), where the design principle has been studied for the one layer case in $[13,14]$. With a long enough taper length (a few millimeters in both layers), the misalignment between the two layers should not affect the coupling ratio in this design.

The fabrication of double layer waveguides is feasible with SiON technology since both core material $(\mathrm{SiON})$ and cladding material $\left(\mathrm{SiO}_{2}\right)$ can be grown on top of each other. In principle, even multiple layers of waveguides are possible with SiON technology. However, the double-layer fabrication process needs to be developed.

The second approach to avoid the waveguide crossings is to detect the light before it 
reaches a crossing. As can be seen in Fig. 6.2.2, if optical detectors can be built on the P-OCT chip, all the waveguides connected to the fiber array to the external detectors can be terminated before the crossings. An InAs/InP(100) quantum dot waveguide photodetector has been demonstrated [15], which could be a candidate for this approach. Another possibility is using integrated micrometer-size $45^{\circ}$ mirrors [16] to reflect the light vertically up into a commercially available detector array which might be glued on the top surface of the P-OCT chip.

The fabrication process in this approach is expected to be more difficult than in the double-layer approach. This is due to the fact that both integrated detectors and $45^{\circ}$ mirrors are realized in different technologies and their key functions are material dependent. One of the possible solutions would be redesigning the P-OCT components in the waveguide photodetector system or the $45^{\circ}$ mirror system, since the OCT function is not material dependent. (But possible larger optical loss in a different material system may affect the image SNR.)

The third approach to avoid the waveguide crossings would be by separating every OCT channel completely by building an individual light source for each channel. A possible candidate for such light sources is an integrated tunable quantum-dot laser which has been developed for OCT applications [17]. This multiple light sources approach can also be combined with the integrated detector approach mentioned above. The combination will result in a chip with only electronic connections, which could be a significant advantage for miniaturizing the complete P-OCT system.

The fabrication difficulties are similar to or larger than in the second approach mentioned above. The possible solutions would be redesigning the P-OCT components in the laser material system. However, multiple lasers and their control structures may interfere with one another.

In conclusion, there are many ways to avoid waveguide crossings in a P-OCT chip. The most suitable choice depends on the application requirements. For instance, in endoscope applications, an electrically passive probe is preferred [18], which makes the double-layer approach the most suitable one. In another case, if a minimal system size is needed for portable applications, the approach with both integrated light sources and detectors might be preferred. In practice, the difficulty of technology development and the cost also plays a role for the design choice, since all mentioned solutions require additional fabrication process development. 


\subsubsection{Suggestions to reduce motion blur with P-OCT}

Motion blur in an OCT image is caused by the relative movement between the OCT system and the sample during the period of imaging. The commonly used solution is to use high A-scan rates such as $70 \sim 300 \mathrm{kHz}$ [19]. However, even with a $300 \mathrm{kHz}$ A-scan rate approximately 1 second is needed to obtain a $512 \times 512$ (A-scans) image. In case of imaging a biological sample in vivo, a $1 \mathrm{~s}$ time span is likely to cause motion blur. Higher A-scan rates of a few $\mathrm{MHz}$ are possible [20]. However, this requires a very fast and high power swept laser source (few $\mathrm{MHz}$ ) and even faster (A-scan rate multiplied with the number of spectral channels in one A-scan) detectors and analog-to-digital converters. In this section, we propose an alternative method to reduce the motion blur (not include sample deformation).

The basic idea is a system with two independent OCTs on the same chip and a closed loop optical feedback control. Two swept lasers are needed, one with faster sweep rate than the other. The faster one should have a sweep speed much faster than the sample movement (such as a few $\mathrm{kHz}$ ). To compensate the sample motion in axial direction, the faster sweep laser is connected to one OCT channel on the P-OCT chip. The slower swept laser (for example a few hundred $\mathrm{Hz}$ ) is connected to the other $N$ channels ( $N=512$ for instance) on the same chip. The P-OCT chip is placed on a piezoelectric or a motor stage, which has a sufficient travel range and speed to compensate the movement relative to the sample. The faster scanning OCT channel is used to monitor the axial motion of the sample. The moving stage is used to compensate the axial motion of the sample. Thus, the $N$ slower channels can keep measuring during this time without being affected by the axial motion of the sample. Note, with a single tracking channel, it only works for the samples without lateral motion or rotation.

In principle, by increasing the number of fast tracking channels to a few, the lateral motion (along the chip edge direction) and rotation (in the chip plane) of the sample can also be tracked and compensated. If a separate tracking chip with few OCT channels is glued orthogonal to the main OCT chip plane, the compensation of sample lateral motion perpendicular to the chip plane and rotation not in the chip plane are possible.

\subsection{Summary}

In this chapter, we briefly introduced the existing parallel OCT systems and their advantage compared with the single beam scanning system. We then presented the design and fabrication results of our common-path based PSS-OCT chip. Several characterization experiments were suggested to investigate the crosstalk between the channels and the effects due to waveguide crossings. After that, we presented a discussion on possible future improvements of the chip design in the following aspects: reduce sampling channel spacing, 
avoid waveguide crossings and reduce motion blur. We believe that there are many opportunities for improving chip-based P-OCT. 


\section{References}

1. Bonin, T., G. Franke, M. Hagen-Eggert, P. Koch, and G. Huttmann, In vivo Fourier-domain full-field OCT of the human retina with 1.5 million A-lines/s. Optics Letters, 2010. 35(20): p. 3432-3434.

2. Dubois, A., G. Moneron, K. Grieve, and A.C. Boccara, Three-dimensional cellular-level imaging using full-field optical coherence tomography. Physics in Medicine and Biology, 2004. 49(7): p. 1227-1234.

3. Ducros, M., M. Laubscher, B. Karamata, S. Bourquin, T. Lasser, and R.P. Salathe, Parallel optical coherence tomography in scattering samples using a two-dimensional smart-pixel detector array. Optics Communications, 2002. 202(1-3): p. 29-35.

4. Dubois, A., J. Moreau, and C. Boccara, Spectroscopic ultrahigh-resolution full-field optical coherence microscopy. Optics Express, 2008. 16(21): p. 17082-17091.

5. Drexler, W. and J.G. Fujimoto, Optical Coherence Tomography: Technology and Applications. 2008: Springer.

6. Grajciar, B., M. Pircher, A.F. Fercher, and R.A. Leitgeb, Parallel Fourier domain optical coherence tomography for in vivo measurement of the human eye. Optics Express, 2005. 13(4): p. 1131-1137.

7. Arauz, L.J., Y. Luo, J.E. Castillo, R.K. Kostuk, and J. Barton, 10-channel fiber array fabrication technique for parallel optical coherence tomography system - art. no. 64292H. Coherence Domain Optical Methods and Optical Coherence Tomography in Biomedicine XI, 2007. 6429: p. H4292-H4292.

8. Luo, Y., L.J. Arauz, J.E. Castillo, J.K. Barton, and R.K. Kostuk, Parallel optical coherence tomography system. Applied Optics, 2007. 46(34): p. 8291-8297.

9. Luo, Y., G. Li, X. Zheng, J. Yao, H. Thacker, J.-H. Lee, J.E. Cunningham, K. Raj, and A.V. Krishnamoorthy, Low-loss low-crosstalk silicon rib waveguide crossing with tapered multimodeinterference design. Crosstalk, 2012. 32(31.5): p. 31.5.

10. Zhang, Y., S.Y. Yang, A.E.J. Lim, G.Q. Lo, C. Galland, T. Baehr-Jones, and M. Hochberg, A CMOSCompatible, Low-Loss, and Low-Crosstalk Silicon Waveguide Crossing. Ieee Photonics Technology Letters, 2013. 25(5): p. 422-425.

11. Bogaerts, W., P. Dumon, D. Van Thourhout, and R. Baets, Low-loss, low-cross-talk crossings for siliconon-insulator nanophotonic waveguides. Optics Letters, 2007. 32(19): p. 2801-2803.

12. Little, B.E. and T. Murphy, Design rules for maximally flat wavelength-insensitive optical power dividers using Mach-Zehnder structures. Ieee Photonics Technology Letters, 1997. 9(12): p. 1607-1609.

13. Akca, B.I., Spectral-domain optical coherence tomography on a silicon chip. 2012.

14. Louisell, W.H., Analysis of the Single Tapered Mode Coupler. Bell System Technical Journal, 1955. 34(4): p. 853-870.

15. Jiao, Y.Q., B.W. Tilma, J. Kotani, R. Notzel, M.K. Smit, S.L. He, and E.A.J.M. Bente, $\operatorname{InAs} / \operatorname{InP}(100)$ quantum dot waveguide photodetectors for swept-source optical coherence tomography around $1.7 \mathrm{mu}$ m. Optics Express, 2012. 20(4): p. 3675-3692.

16. Civitci, F., G. Sengo, A. Driessen, M. Pollnau, A.J. Annema, and H.J.W.M. Hoekstra, Light turning mirrors for hybrid integration of SiON-based optical waveguides and photo-detectors. Optics Express, 2013. 21(20): p. 24375-24384.

17. Tilma, B.W., Y.Q. Jiao, J. Kotani, B. Smalbrugge, H.P.M.M. Ambrosius, P.J. Thijs, X.J.M. Leijtens, R. Notzel, M.K. Smit, and E.A.J.M. Bente, Integrated Tunable Quantum-Dot Laser for Optical Coherence Tomography in the $1.7 \mathrm{mu} \mathrm{m}$ Wavelength Region. Ieee Journal of Quantum Electronics, 2012. 48(2): p. 87-98.

18. Ford, H.D. and R.P. Tatam, Coherent Fibre Bundles in full-field swept-source OCT. Optical Coherence Tomography and Coherence Domain Optical Methods in Biomedicine Xiii, 2009. 7168.

19. Potsaid, B., I. Gorczynska, V.J. Srinivasan, Y.L. Chen, J. Jiang, A. Cable, and J.G. Fujimoto, Ultrahigh speed Spectral/Fourier domain OCT ophthalmic imaging at 70,000 to 312,500 axial scans per second. Optics Express, 2008. 16(19): p. 15149-15169. 
20. Wieser, W., B.R. Biedermann, T. Klein, C.M. Eigenwillig, and R. Huber, Multi-Megahertz OCT: High quality $3 D$ imaging at 20 million A-scans and 4.5 GVoxels per second. Optics Express, 2010. 18(14): p. 14685-14704. 


\section{Chapter 7}

\section{Conclusions and outlook}

\subsection{Conclusions}

The goal of this study is to develop an OCT chip with external light source and detector. The motivation of developing such a system is that the chip-based OCT systems have the potential for considerable size and cost reduction compared with the bulky free-space- or fiber-based systems.

We presented a literature survey of existing chip-based OCT system in Chapter 1. It is shown that even though a lot of progress has been made in the chip-based OCT studies in recent years, there are still several common problems. All these studies are dual-arm OCT systems, where space consuming and dispersive reference arm are needed. They also need external lenses for coupling between the chip and the sample. We propose a common-path configuration with an on-chip micro-ball lens instead of a dual arm design. This commonpath configuration uses the Fresnel reflection from the end facet of the waveguide as the reference reflector instead of the relative large and dispersive reference arm. To our best knowledge, the smallest existing dual arm OCT systems has a chip area of $0.75 \times 5 \mathrm{~mm}^{2}$ (excluding the lenses between the chip and the sample, see Table 1.3.1). By eliminating the needs of a separate reference arm, our common-path design can have a chip area as small as $\sim 0.1 \times 0.4 \mathrm{~mm}^{2}$ (including the lens between the chip and the sample see Section 5.7). The dramatic reduction in the chip area enables the designing of chip-based parallel OCT (P-OCT) systems with a large number of OCT channels. It was argued that the disadvantage of the common-path configuration is that the sample must be very close (within the maximum imaging depth $z_{\max }$ ) to the chip, which may lead to some limitations in application.

In order to better understand the working principle of the OCT system, we carefully studied the mathematical model of the Fourier-domain OCT (FD-OCT) system. During the study, we found that existing models (to the best of our knowledge) are mainly based on free-space- or fiber-based OCT systems, where optical losses are negligible compared with chip-based systems. Therefore, we extended the existing model by including transmittance factors of different elements in an OCT system. The influence to the signal-to-noise ratio 
(SNR) by these transmittance factors at different power and noise conditions are summarized in Table 2.6.1. The quantitative analysis of the influence of the transmittance factors on the SNR are particularly useful during the optimization of the optical components. For instance, a lower loss waveguide component will not improve the SNR for a system where its noise is dominated by the relative intensity noise (RIN) of the light source. Thus, no further optimization of reducing losses of the waveguide elements are needed for improving the SNR in that case. The example mentioned above is suitable for improving an existing system, where the noise situation and sample-reference power relation are known. However, in case of designing a new system such as in this study, the exact noise situation is unknown. Thus, we can only try to reduce the optical losses in all components, since in the majority of cases, it will improve the SNR. Therefore we use adiabatic bends and modified Y junctions in order to reduce the optical losses in the chip (see Chapter 3).

A broad band directional coupler is an alternative for the $\mathrm{Y}$ junction (see Chapter 6). The directional coupler has slightly lower loss compared with the $\mathrm{Y}$ junction, but has much larger wavelength dependency and is sensitive to fabrication errors. In principle, a properly designed broad band directional coupler can have better SNR than a Y junction in a certain wavelength length range. In practice, the fabrication accuracy largely determines which one is better. If the loss and wavelength dependency of both $\mathrm{Y}$ junction and directional coupler are known, Fig. 2.6.1 can be used to estimate which one results in a higher SNR. A low loss broad band circulator is another alternative to the Y junction. However, the existing on-chip circulator technology is not compatible with our SiON waveguide technology at this moment. A hybrid integration, such as chip bonding may lead to a solution.

One of the common problems in chip-based OCTs is the large divergence of the light exit from the waveguide. In this study, we developed a technique to directly fabricate micro-ball lenses on the optical chip to reduce the light divergence (reduction in the half divergence angle from $13.2^{\circ}$ to $1.56^{\circ}$ at a wavelength of $1306 \mathrm{~nm}$ and from $12.4^{\circ}$ to $1.85^{\circ}$ at a wavelength of $1544 \mathrm{~nm}$, see Chapter 4) in both horizontal and vertical direction. This micro-ball lens is not limited to chip-based OCT application. It can be applied in many other optical chip based applications where reduced beam divergence or even focused beams are required. The lens fabrication is based on photolithography and thermal reflow, which are in principle suitable for mass production.

The micro-ball lens ( $100 \mu \mathrm{m}$ diameter) is an important element in our common-path OCT system. The small dimension of the lens and its short focal length ensures that the distance between the reference plane (waveguide end facet) and the sample can be smaller than the maximum imaging depth. On the other hand, this lens also has Fresnel reflections, which leads to two additional reference planes. These additional references produce ghost images in an OCT A-scan. There are two possible ways to solve this problem. First, using antireflection coating on the lens. However, it is technically challenging to coat such a lens. 
Second, we present a mathematical model of a multi-reference OCT in Section 2.3. Based on this model, we found that these ghost images can be suppressed with a deconvolution algorithm. We believe that the developed deconvolution approach, used to eliminate the influence of multi-reference planes, is applicable in many other OCT systems, such as a fiber-based common-path OCT with a small ball lens [1]. The deconvolution algorithm is also able to slightly improve the axial resolution, which may also be used to sharpen the OCT image in the axial direction.

The performance of a single channel OCT is demonstrated in Chapter 5. A comparison measurement shows that the presence of the micro-ball lens improves the SNR with up to $37 \mathrm{~dB}$ compared with the chip without a micro-ball lens. An optical phantom, that is used to mimic the skin, was imaged with our chip-based OCT. The layered structures are clearly visible and the deconvolution algorithm shows clear suppression of the ghost images. The demonstration of the single channel OCT indicates that this system is ready for real sample measurement in the near future.

An 8-channel P-OCT chip has been fabricated. Due to the time limitation, a full characterization of this chip is not yet performed. We are interested in the crosstalk between different OCT channels. Several experiments are suggested to investigate the crosstalk with this chip.

In conclusion, we reached our goal by demonstrating a chip-based single channel OCT. We believe that many more studies are needed to further improve the chip-based OCT system. Some suggestions are discussed in the outlook section (Section 7.2). Further studies on chipbased P-OCT are exciting, since it is very new in this field and may open up new possibilities for system design and applications.

\subsection{Outlook}

We have demonstrated a chip-based common-path SS-OCT system. There are certainly motivations for continuing to work in this field, since several challenges remain on the road towards a reliable, parallelized and fully integrated handhold OCT probe.

Firstly, the micro-ball lens fabrication process should be further improved in order to increase the fabrication yield. The current yield is $85 \%$, which is mainly limited by the present of cooling water during the dicing procedure. The way to overcome this limitation is by reducing the dicing time (with a better dicing pattern design) or by using a dry dicing machine (avoid the cooling water completely).

Secondly, investigation of the feasibility of fabricating the micro-ball lens using different materials, such as other photoresists, may further widen the applicable field of this microlens technology. For instance, applications in organic solvent environment require a 
lens material that does not dissolve in the solvent (the current used AZ 9260 resist will be dissolved in organic solvents).

Thirdly, since our chip has been designed to be wavelength insensitive in a much larger wavelength range than the swept laser can offer (1266-1358 nm), we are planning to use a SD-OCT configuration (broad band light source plus spectrometer) in the future to study the performance of the chip in a wavelength range of 1.2-1.7 $\mu \mathrm{m}$. This much larger wavelength range is very interesting for high axial resolution OCT.

Fourthly, the B-scans demonstrated in Chapter 5 were obtained by translating the sample. Since the micro-ball lens is integrated and the optical chip is a small low-mass device, an alternative implementation might mount the fiber-connected chip directly on a scanner to obtain two- or three-dimensional images.

Fifthly, a P-OCT chip is fabricated, however, its characterization is not complete yet. Experiments as suggested in Chapter 6 should be done in the near future to investigate its performance and the possible crosstalk effects between different OCT channels. These experiments are expected to be valuable for improving the P-OCT chip design in the future.

Finally, it is necessary to integrate the light source and detector onto the chip for a fully integrated OCT chip. There are studies that attempted to integrate on-chip tunable laser sources [2] and waveguide photodetectors [3] into chip-based SS-OCT systems. These components are based on technology which is not compatible with our SiON technology. However, the lens fabrication and $\mathrm{Y}$ junction design can be adapted into the InAs/InP technology, which makes possible for the full integration. The total waveguide length required in our common-path OCT configuration is very short (in the order of few hundreds of micrometers or less), thus the total waveguide loss is expected to be small even in high loss waveguides (such as InAs/InP based waveguides).

We believe that there are many more opportunities for improving chip-based OCT system than the ones mentioned here. The development of miniaturized, stable, maintenance free and mass producible chip-based OCT systems have the potential for considerable size and cost reduction which may lead to wider applications in both medical and industrial fields. 


\section{References}

1. Zhao, M.T., Y. Huang, and J.U. Kang, Sapphire ball lensed fiber probe for common-path optical coherence tomography in ocular imaging and sensing. Ophthalmic Technologies Xxiii, 2013. 8567.

2. Tilma, B.W., Y.Q. Jiao, J. Kotani, B. Smalbrugge, H.P.M.M. Ambrosius, P.J. Thijs, X.J.M. Leijtens, R. Notzel, M.K. Smit, and E.A.J.M. Bente, Integrated Tunable Quantum-Dot Laser for Optical Coherence Tomography in the $1.7 \mathrm{mu} \mathrm{m}$ Wavelength Region. Ieee Journal of Quantum Electronics, 2012. 48(2): p. $87-98$

3. Jiao, Y.Q., B.W. Tilma, J. Kotani, R. Notzel, M.K. Smit, S.L. He, and E.A.J.M. Bente, InAs/InP(100) quantum dot waveguide photodetectors for swept-source optical coherence tomography around $1.7 \mathrm{mu}$ m. Optics Express, 2012. 20(4): p. 3675-3692. 


\section{Appendices}

\section{Fabrication processes}

\section{A.1 Introduction}

In this appendix, we proved a detailed description of the fabrication processes for the realization of the OCT-chip. It includes an overview of the mask (section A.2) and the fabrication steps with their process parameters (section A.3).

\section{A.2 Mask layout}

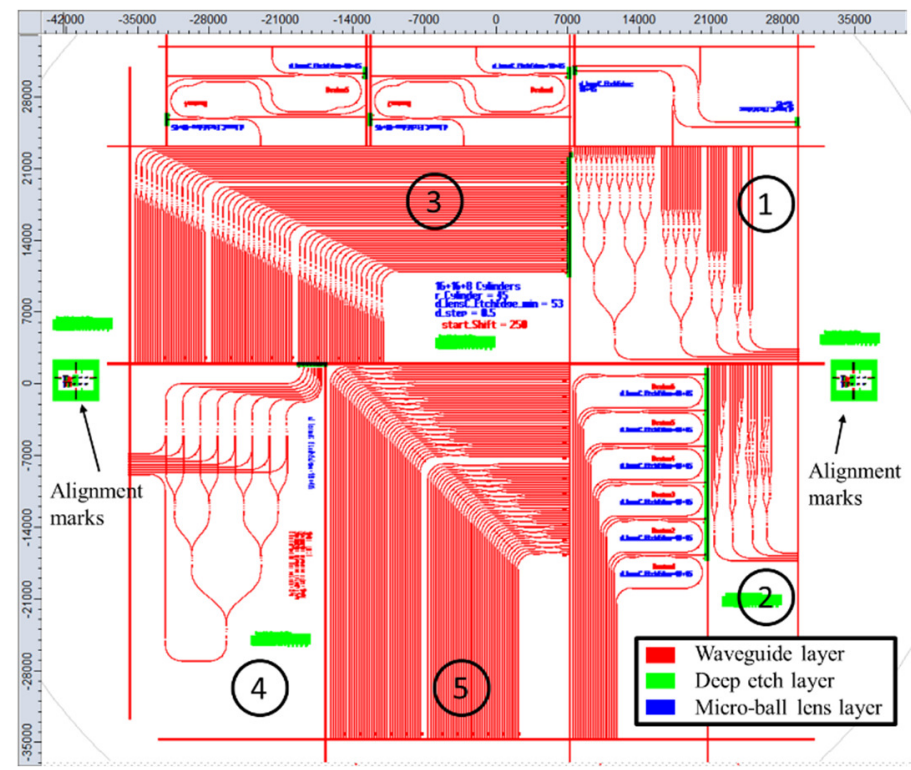

Fig. A1. Mask layout overview. The numbers refer to different section (see text for an explanation). 
The mask layout overview is shown in Fig. A1. There are 5 sections (marked with numbers in Fig. A1) that contain the structures that have been described in the thesis. The other sections (unmarked) contain testing structures that are not included in this thesis. The marked sections are described in the following chapters:

1. Used to measure the power ratio (splitting ratio) of the $\mathrm{Y}$ junction in Chapter 3.

2. Used to measure the optical loss of the Y junction in Chapter 3.

3. Used to characterize the beam shape behand the micro-ball lens in Chapter 4. It is also used to perform the single channel OCT measurements in Chapter 5.

4. The parallel OCT device described in Chapter 6 (not fully characterized yet).

5. The single layer Mach-Zehnder couplers with optimal phase delay for broad band 50/50 coupling described in Chapter 6 (not characterized yet).

The layout shown in Fig. A1 has three separate masks (three layers), namely: (i) waveguide mask; (ii) deep etch mask and (iii) micro-ball lens mask. The functions of these three masks are illustrated in Fig. A2.

(a)

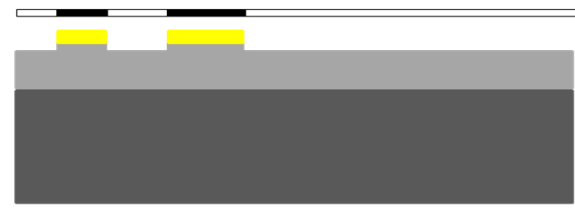

(b)

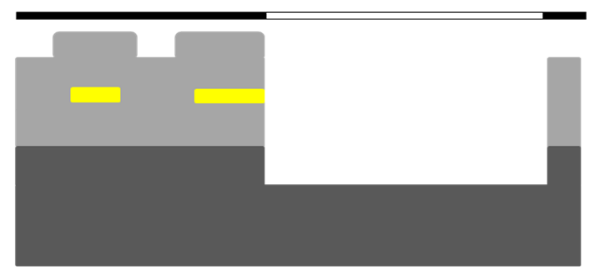

(c)

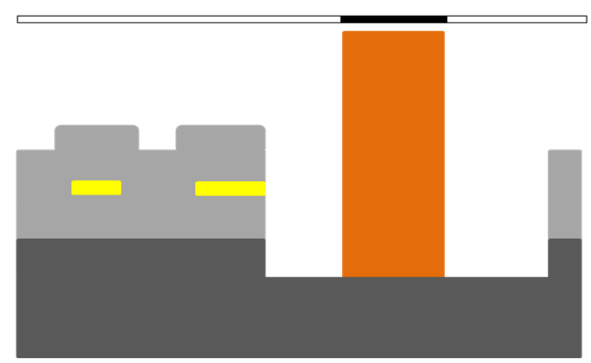

Waveguide Mask

\section{PECVD SiON}

Thermal $\mathrm{SiO}_{2}$

$\mathrm{Si}$

Deep Etch Mask

PECVD $\left(\mathrm{SiO}_{2}\right)$

TEOS $\left(\mathrm{SiO}_{2}\right)$

PECVD SiON

Thermal $\mathrm{SiO}_{2}$

$\mathrm{Si}$

Micro-ball lens Mask

Photoresist

PECVD $\left(\mathrm{SiO}_{2}\right)$

TEOS $\left(\mathrm{SiO}_{2}\right)$

PECVD SiON

Thermal $\mathrm{SiO}_{2}$

$\mathrm{Si}$

Fig. A2. The functions of the masks. (a) The waveguide mask is a high resolution laser mask or an E-beam mask which is used for patterning the waveguide structures. (b) The deep etch mask is a laser mask (relative lower resolution and cheaper compared with the waveguide mask) which is used for patterning the platform of the microlens. (c) The Micro-ball lens mask is a laser mask used for patterning the thick photoresist of the microlens. 


\section{A.3 Fabrication process parameters}

In this section, we describe all the fabrications processes in our device. All these processes are shown in Fig. A.3 (SiON waveguide fabrication), Fig. A.4 (platform fabrication) and Fig. A.5 (Micro-ball lens fabrication). The corresponding process parameters are listed in Table A.1.

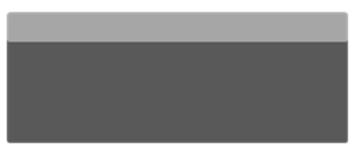

1. Si wafer with $8 \mu \mathrm{m}$ thermal $\mathrm{SiO}_{2}$ layer: Step 1-3

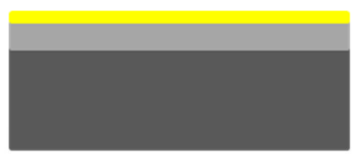

2. Grow SiON layer ( $600 \mathrm{~nm}$ thick) and anneal: Step 4-31

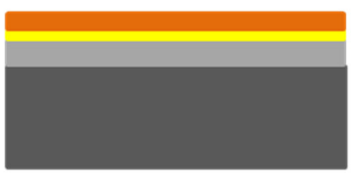

3. Spin coat photoresist $906-12$ $(1.2 \mu \mathrm{m})$ : Step 32-40

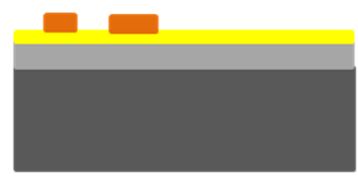

4. Photolithography: Step 41-47

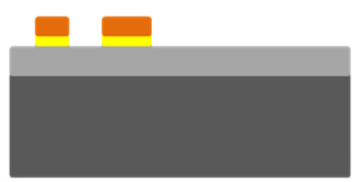

5. RIE etch optical waveguide: Step 48-49

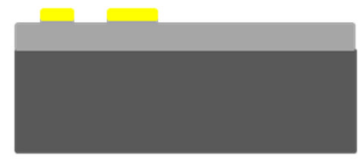

6. Stripping resist in oxygen plasma: Step 50-51

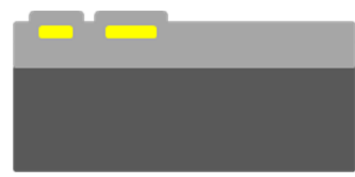

7. Grow TEOS layer $(1 \mu \mathrm{m}$ $\mathrm{SiO}_{2}$ ) and anneal: Step 52-69

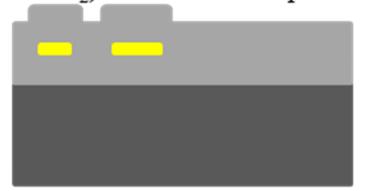

8. Grow PECVD $\mathrm{SiO}_{2}$ layer (3 $\mu \mathrm{m})$ and anneal: Step 70-88

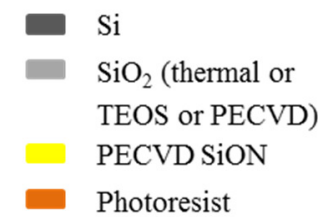

Fig. A.3. SiON waveguide fabrication. There are 8 processes to accomplish the waveguide fabrication (with $\mathrm{SiO}_{2}$ top cladding). Each process has several steps and the step numbers are referred to the step number in Table A.1. 


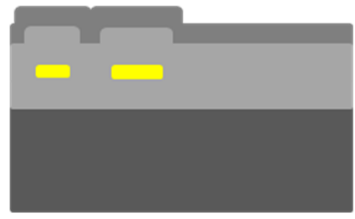

1. Grow $1.1 \mu \mathrm{m}$ LPCVD polysilicon layer: Step 89-98

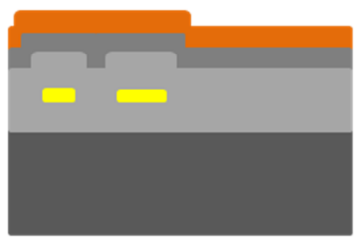

2. Spin coat photoresist $907-17$ $(1.7 \mu \mathrm{m})$ : Step $99-106$

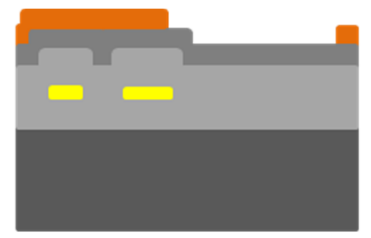

3. Photolithography: Step $107-$ 112

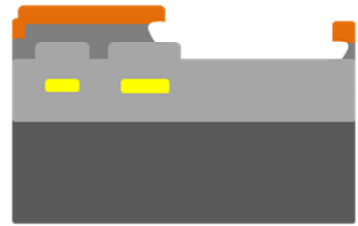

4. RIE etch Poly silicon: Step 113

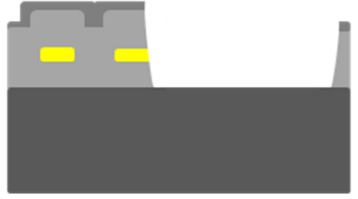

5. RIE etch $\mathrm{SiO}_{2}$ : Step 114-116

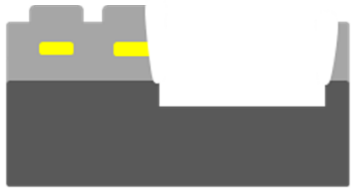

6. RIE etch Si: Step 117-122

Photoresist

$\mathrm{Si} \mathrm{SiO}_{2}$ (thermal or TEOS or PECVD)

PECVD SiON

Poly-silicon

Fig. A.4. Platform fabrication.

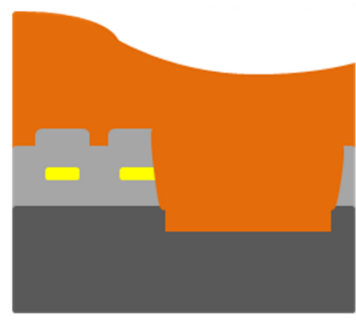

1. Spin coat $50 \sim 60 \mu \mathrm{m}$ photoresist AZ9260: Step 123-133

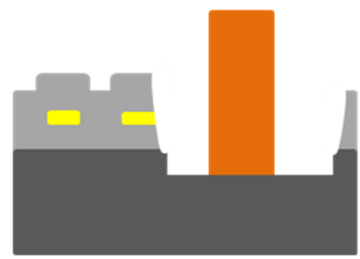

2. Photolithography: Step 134138

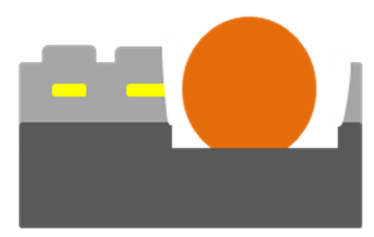

3. Reflow: Step 139-140

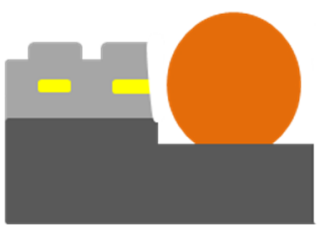

4. Dice and wash with water: Step 141-142

Fig. A.5. Micro-ball lens fabrication. 
Table A.1. Process parameters. All the steps are performed in the cleanroom of MESA+. The corresponding instruments are listed at the end of this table.

\section{Grow SiON layer}

\begin{tabular}{|c|c|c|c|}
\hline Step & \multicolumn{2}{|c|}{ Process } & Comments \\
\hline \multirow[t]{5}{*}{1} & \multicolumn{2}{|c|}{ Take wafers: } & \multirow{5}{*}{$\begin{array}{l}\text { Take } 10 \text { wafers (used for dummy grows during the } \\
\text { SiON layer deposition) from Wafer Storage } \\
\text { Cupboard }\end{array}$} \\
\hline & Substrate: silicon; & Orientation: <100>; & \\
\hline & Thickness: $525 \pm 25 \mu \mathrm{m}$ & Diameter: 100 mm; & \\
\hline & Resistivity: 5-10 $\Omega \mathrm{cm}$ & Type: p; & \\
\hline & Single side polished & & \\
\hline \multirow[t]{5}{*}{2} & \multicolumn{2}{|c|}{ Take wafers: } & \multirow{5}{*}{$\begin{array}{l}\text { Take } 10 \text { wafers (dummy wafers plus device } \\
\text { wafers) from Wafer Storage Cupboard }\end{array}$} \\
\hline & Substrate: silicon; & Orientation: $<100>$; & \\
\hline & Thickness: $525 \pm 25 \mu \mathrm{m}$ & Diameter: 100 mm; & \\
\hline & Resistivity: 5-10 $\Omega \mathrm{cm}$ & Type: p; & \\
\hline & Single side polished & $8 \mu \mathrm{m}$ thermal $\mathrm{SiO}_{2}$ & \\
\hline 3 & \multicolumn{2}{|c|}{ Ellipsometer measurement ${ }^{\mathrm{a}}$} & $\begin{array}{l}\text { Measure the thermal } \mathrm{SiO}_{2} \text { thickness of every } \\
\text { wafer. (Will be used to measure the thickness of } \\
\text { SiON layer on each wafer.) }\end{array}$ \\
\hline $4-10$ & \multicolumn{2}{|c|}{ Standard wafer cleaning* } & \\
\hline 11 & \multicolumn{2}{|c|}{ Predeposition Oxford 80 } & Clean the chamber \\
\hline 12 & \multicolumn{2}{|c|}{ Predeposition Oxford 80 } & $\begin{array}{l}\text { Grow } 1 \mu \mathrm{m} \text { low refractive index }(n) \mathrm{SiON} \text { to the } \\
\text { chamber without wafer }\end{array}$ \\
\hline \multirow[t]{4}{*}{13} & \multicolumn{2}{|c|}{ Test deposition SiON layer ${ }^{\mathrm{b}}$ : } & \multirow{4}{*}{$\begin{array}{l}\text { Use Oxford } 80 \text { machine } \\
\text { Speed is about } 40.9 \mathrm{~nm} / \mathrm{min} \text {. } \\
\text { Grown } 300 \mathrm{~nm} \mathrm{SiON} \mathrm{layers} \mathrm{on} \mathrm{Si} \text {. }\end{array}$} \\
\hline & Frequency: $187.5 \mathrm{kHz}$ & Power: $60 \mathrm{~W}$ & \\
\hline & Pressure: 460 mToor; & Temperature: $300^{\circ} \mathrm{C}$; & \\
\hline & $\mathrm{N}_{2} \mathrm{O}: 440 \mathrm{sccm}$ (variable) & $\mathrm{SiH}_{4} / \mathrm{N}_{2}: 1000 \mathrm{sccm}$ & \\
\hline 14 & \multicolumn{2}{|c|}{ Ellipsometer measurement ${ }^{\mathbf{a}}$} & $\begin{array}{l}\text { Measure thickness } d, n(A n, B n \text { are the dispersion } \\
\text { coefficient used in the machine) and uniformity. If } \\
\text { refractive index uniformity } d n<0.001 \text { and } \\
\text { thickness uniformity } d \text { thickness }<2 \% \text {, go on. } \\
\text { Otherwise, stop and check the problem. }\end{array}$ \\
\hline 15 & & & $\begin{array}{l}\text { Loop the last two steps to tune }\left(\mathrm{SiH}_{4}: \mathrm{N}_{2} / \mathrm{N}_{2} \mathrm{O}\right) \\
\text { refractive index } n \text { and find grown rate } d / \mathrm{min}\end{array}$ \\
\hline 16 & Predepositiol & Oxford 80 & Clean the chamber \\
\hline
\end{tabular}




\begin{tabular}{|c|c|c|c|}
\hline Step & \multicolumn{2}{|c|}{ Process } & Comments \\
\hline 17 & \multicolumn{2}{|c|}{ Predeposition Oxford $\mathbf{8 0}^{\mathrm{b}}$} & $\begin{array}{l}\text { Grow } 1 \mu \mathrm{m} \text { low } n \text { SiON to the chamber without } \\
\text { wafer }\end{array}$ \\
\hline 18 & \multicolumn{2}{|c|}{$\begin{array}{l}\text { Dummy deposition }^{\mathbf{b}} \\
\text { (Use parameters from step 13) }\end{array}$} & $\begin{array}{l}\text { Grow } 625 \mathrm{~nm} \text { thick SiON layers on Si. This means } \\
\text { a thickness of } 600 \mathrm{~nm} \text { after anneal. } \\
{[600 \mathrm{~nm} / \text { anneal_factor }=600 / 0.96=625 \mathrm{~nm} \text {. This }} \\
\text { anneal_factor }(0.96) \text { is for } n=1.55 \text { at wavelength of } \\
\left.1300 \mathrm{~nm} \text { annealed at } 1100^{\circ} \mathrm{C} \text {. }\right]\end{array}$ \\
\hline 19 & \multicolumn{2}{|c|}{ Ellipsometer measurement $^{\mathrm{a}}$} & $\begin{array}{l}\text { Measure } d, n(A n, B n) \text { (may use fixed } A n, B n \text { from } \\
\text { test deposition) and uniformity. If } d n<0.001 \text { and } \\
d \text { _thickness }<2 \% \text {, go on. Otherwise, stop and } \\
\text { check the problem. }\end{array}$ \\
\hline 20 & \multicolumn{2}{|c|}{$\begin{array}{l}\text { Device wafer deposition } \\
\text { (Use parameters from step } 13 \text { ) }\end{array}$} & $\begin{array}{l}\text { Grow } 625 \mathrm{~nm} \text { thick PECVD SiON layers on } 8 \mu \mathrm{m} \\
\text { thermal } \mathrm{SiO}_{2} \text {. Grow one wafer each time. }\end{array}$ \\
\hline 21 & \multicolumn{2}{|c|}{ Ellipsometer measurement ${ }^{\mathrm{a}}$} & $\begin{array}{l}\text { Measure } d \_S i O N \text {, (use fixed thermal } \mathrm{SiO}_{2} \text { layer } \\
\text { data, and fixed } \mathrm{SiON} A n, B n \text { from dummy } \\
\text { deposition) and uniformity. If } d n<0.001 \text { and } \\
d \text { _thickness }<2 \% \text {, go on. Otherwise, stop and } \\
\text { check the problem. }\end{array}$ \\
\hline 22 & & & $\begin{array}{l}\text { Loop from the predeposition (step 16): } 1 \text { dummy, } \\
3 \text { device wafers. If one wafer is wrong, start the } \\
\text { loop again. If there still something wrong, stop and } \\
\text { check the problem. }\end{array}$ \\
\hline $23-29$ & \multicolumn{2}{|c|}{ Standard wafer cleaning* } & \\
\hline \multirow[t]{5}{*}{30} & \multicolumn{2}{|c|}{ Annealing: } & \multirow[t]{5}{*}{ Use Furnace H1 or A (not B3), 3 hours } \\
\hline & Standby T: $800^{\circ} \mathrm{C}$ & Program: ANN1100C; & \\
\hline & $\mathrm{T}: 1100^{\circ} \mathrm{C}$ & Time: 3 hours; & \\
\hline & Gas: $\mathrm{N}_{2}$; & Flow: $1 \mathrm{~L} / \mathrm{min}$ & \\
\hline & Ramp: $10^{\circ} \mathrm{C} / \mathrm{min}$ & Cool down: $7.5^{\circ} \mathrm{C} / \mathrm{min}$ & \\
\hline 31 & \multicolumn{2}{|c|}{ Ellipsometer measurement $^{\mathrm{a}}$} & $\begin{array}{l}\text { Measure } d, n(A n, B n) \text { and uniformity on all wafers } \\
\text { (dummy first, then use the fixed } A n, B n \text { from } \\
\text { dummy) }\end{array}$ \\
\hline
\end{tabular}

\section{Pattern waveguide}

\begin{tabular}{|c|c|l|}
\hline Step & Process & \multicolumn{1}{c|}{ Comments } \\
\hline $32-36$ & Standard wafer cleaning* & Steps 1, 2, 5, 6, and 7 \\
\hline $37-38$ & Apply HMDS adhesion layer** & \\
\hline
\end{tabular}




\begin{tabular}{|c|c|c|c|}
\hline Step & \multicolumn{2}{|c|}{ Process } & Comments \\
\hline 39 & \multicolumn{2}{|c|}{$\begin{array}{l}\text { Coating photoresist Olin Oir 906-12: } \\
\text { Spin program: } 4000(4000 \mathrm{rpm}, 30 \mathrm{sec}) \\
\text { Prebake: hotplate, T: } 95^{\circ} \mathrm{C} \text {, time } 60 \mathrm{~s}\end{array}$} & Use primus Spinner in wet bench 21 \\
\hline 40 & \multicolumn{2}{|c|}{$\begin{array}{l}\text { Coating AZ Aquatar: } \\
\text { Spin program: } 2200 \mathrm{rpm}, 30 \text { seconds }\end{array}$} & $\begin{array}{l}\text { Wet bench } 19 \text { (for polymer) } \\
\text { Antireflection and anti-sticky }\end{array}$ \\
\hline 41 & \multicolumn{2}{|c|}{$\begin{array}{l}\text { Alignment \& exposure Olin 906-12'} \text { : } \\
\text { Electronic Vision Group EVG620 Mask Aligner, } \\
\text { exposure time: } 3.3 \mathrm{~s}\left(\mathrm{Hg} \text { lamp: } 12 \mathrm{~mW} / \mathrm{cm}^{2}\right)\end{array}$} & $\begin{array}{l}\text { Vacuum mode, center both stage before alignment, } \\
\text { check pressure should <- } 0.8 \text {. After vacuum } \\
\text { contact, Waite } 1 \text { min before expose. }\end{array}$ \\
\hline 42 & \multicolumn{2}{|c|}{$\begin{array}{l}\text { Remove AZ Aquatar before baking: } \\
\text { Immerge in } \mathrm{H}_{2} \mathrm{O} \text { (10 seconds), then spin dry }\end{array}$} & \\
\hline 43 & \multicolumn{2}{|c|}{$\begin{array}{c}\text { Development Olin Oir resist: } \\
\text { After exposure bake on hotplate } \\
\mathrm{T}: 120^{\circ} \mathrm{C} \text {, time } 60 \mathrm{sec} \\
\text { Development in developer: OPD } 4262 \\
\text { In beaker } 1,30 \mathrm{~s} \text {, then in beaker } 2,15-30 \mathrm{~s}\end{array}$} & Wet bench 21 \\
\hline $44-45$ & \multicolumn{2}{|c|}{ Standard wafer cleaning* } & Step 3 and 6 \\
\hline 46 & \multicolumn{2}{|c|}{$\begin{array}{l}\text { Post bake (hard bake) Olin Oir resist: } \\
\text { On hotplate, } \mathrm{T}: 120^{\circ} \mathrm{C} \text {, time: } 30 \mathrm{~min}\end{array}$} & \\
\hline 47 & \multicolumn{2}{|c|}{ Inspection by optical microscope } & $\begin{array}{l}\text { A Nikon Microscope which is dedicated for } \\
\text { lithography inspection. }\end{array}$ \\
\hline \multirow[t]{4}{*}{48} & \multicolumn{2}{|c|}{ RIE etching SiON (PT 790) ${ }^{\mathrm{d}}$ : } & \multirow{4}{*}{$\begin{array}{l}\text { For } 600 \mathrm{~nm} \mathrm{SiON} \mathrm{layer,} \mathrm{etch} \mathrm{time} \mathrm{is} 16 \mathrm{~min} \text {. (get } \\
40 \text { to } 70 \mathrm{~nm} \text { over etch) About } 43 \mathrm{~nm} / \mathrm{min} \text { for not } \\
\text { annealed } \mathrm{SiON} \text {. }\end{array}$} \\
\hline & $\mathrm{T}: 20^{\circ} \mathrm{C}$ & $\mathrm{CHF}_{3}: 100$ & \\
\hline & $\mathrm{O}_{2}: 2$ & Power: $350 \mathrm{~W}$; & \\
\hline & Pressure 30 mTorr & & \\
\hline 49 & \multicolumn{2}{|c|}{$\begin{array}{c}\text { Surface profile measurement }{ }^{\mathrm{e}} \text { : } \\
\text { Use Dektak } 8\end{array}$} & $\begin{array}{l}\text { For } 600 \mathrm{~nm} \text { SiON waveguide: If height }<1620 \mathrm{~nm} \\
\text { then back to step } 48 \text { for further etch. If } \\
\text { height }>1620 \mathrm{~nm} \text { then SiON is just etched } \\
\text { through, if height }>1690 \mathrm{~nm} \text { means with } 100 \mathrm{~nm} \\
\text { over etch. Measure } 5 \text { positions but do not measure } \\
\text { on the waveguide. (This measurement damage } \\
\text { some of the photoresist on top.) }\end{array}$ \\
\hline \multirow[t]{3}{*}{50} & \multicolumn{2}{|c|}{ Strip off resist in oxygen plasma ${ }^{\mathrm{f}}$ : } & \multirow{3}{*}{$\begin{array}{l}\text { Use Tepla300. Barrel Etcher }(2.45 \mathrm{GHz}) \text {. } \\
\text { Ultra clean system only (no metals except Al). }\end{array}$} \\
\hline & $\mathrm{O}_{2}: 200 \mathrm{sccm}(50 \%)$ & Power: up to $1000 \mathrm{~W}$; & \\
\hline & Pressure: 1 mbar; & Time: 30 mins (default) & \\
\hline 51 & \multicolumn{2}{|c|}{$\begin{array}{c}\text { Surface profile measurement }{ }^{\mathrm{e}} \text { : } \\
\text { Use Dektak } 8\end{array}$} & $\begin{array}{l}\text { To confirm the } \mathrm{SiON} \text { layer is etched through. } \\
\text { Calculate etch rate of photoresist and } \mathrm{SiON} \text { layer }\end{array}$ \\
\hline
\end{tabular}


Grow top cladding

\begin{tabular}{|c|c|c|c|}
\hline Step & \multicolumn{2}{|c|}{ Process } & Comments \\
\hline $52-58$ & \multicolumn{2}{|c|}{ Standard wafer cleaning* } & $\begin{array}{l}\text { Take } 2 \text { dummy } \mathrm{Si} \text { wafers for tetraethyl } \\
\text { orthosilicate (TEOS) grow. (one will be annealed } \\
\text { with the device wafers) }\end{array}$ \\
\hline \multirow[t]{4}{*}{59} & \multicolumn{2}{|c|}{$\mathrm{LPCVD} \mathrm{SiO}_{2}$ (TEOS) layer grow: } & \multirow{4}{*}{$\begin{array}{l}\text { TEOS } \mathrm{SiO}_{2} 1100 \mathrm{~nm} \text { (after anneal becomes } \\
1000 \mathrm{~nm} \text { ) in furnace } \mathrm{H} 3 \text {. Deposition rate about } \\
10 \mathrm{~nm} / \mathrm{min}(1100 \mathrm{~nm} \text { ). Wafer non-uniformity: } \\
3.6 \% \text {. Boat non-uniformity: } 1.0 \% \text {. } \mathrm{N}_{\mathrm{f}}: 1.434 \text {. } \\
\text { Stress: } 282 \pm 13 \mathrm{MPa} \text { (after anneal @ } 1150^{\circ} \mathrm{C}, 3 \mathrm{hr} \text { ). }\end{array}$} \\
\hline & Program: $\mathrm{N}_{2}$ & TEOS flow: $40 \mathrm{sccm}$; & \\
\hline & Bubbler $\mathrm{N}_{2}: 30 \mathrm{sccm}$; & $\begin{array}{l}\mathrm{T}: 710^{\circ} \mathrm{C}(\mathrm{Z} 1), 725^{\circ} \mathrm{C} \\
(\mathrm{Z} 2), 740^{\circ} \mathrm{C}(\mathrm{Z} 3) ;\end{array}$ & \\
\hline & Pressure: 400 mTorr; & Time: $1 \mathrm{~h} 50 \mathrm{~min}$ & \\
\hline 60 & \multicolumn{2}{|c|}{ Ellipsometer measurement ${ }^{\mathrm{a}}$} & Dummy TEOS $d, n(A n, B n)$ and uniformity \\
\hline $61-67$ & \multicolumn{2}{|c|}{ Standard wafer cleaning* } & $\begin{array}{l}\text { This is only needed if the waiting/loading of the } \\
\text { wafers takes longer than } 4 \text { hours. }\end{array}$ \\
\hline 68 & \multicolumn{2}{|c|}{$\begin{array}{l}\text { Annealing of the TEOS layer: } \\
\mathrm{T}: 1100^{\circ} \mathrm{C}, \mathrm{N}_{2} \text { flow, } 3 \text { hours }\end{array}$} & $\begin{array}{l}\text { Use } \mathrm{H} 1 \text { furnace. (Should be the same day as the } \\
\text { grows) }\end{array}$ \\
\hline 69 & \multicolumn{2}{|c|}{ Ellipsometer measurement ${ }^{\mathrm{a}}$} & Dummy TEOS after anneal ( $d, n$ and uniformity) \\
\hline $70-76$ & \multicolumn{2}{|c|}{ Standard wafer cleaning* } & $\begin{array}{l}\text { This is only needed if the waiting/loading of the } \\
\text { wafers takes longer than } 4 \text { hours. }\end{array}$ \\
\hline 77 & \multicolumn{2}{|c|}{ Predeposition Oxford 133 } & Clean the chamber \\
\hline \multirow[t]{4}{*}{78} & \multicolumn{2}{|c|}{ Predeposition $\mathrm{SiO}_{2}$ with Oxford 133 $^{\mathrm{g}}$ : } & \multirow{4}{*}{$\begin{array}{l}\text { Grown } 1 \mu \mathrm{m} \text { PECVD } \mathrm{SiO}_{2} \text { to the chamber without } \\
\text { wafer. }\end{array}$} \\
\hline & LF Power: $60 \mathrm{~W}$; & Pressure: 650 mTorr; & \\
\hline & $\mathrm{T}: 300{ }^{\circ} \mathrm{C}$ & $\mathrm{N}_{2} \mathrm{O}$ flow: $710 \mathrm{sccm}$ & \\
\hline & $2 \% \mathrm{SiH} / \mathrm{N}_{2}: 200 \mathrm{sccm}$ & & \\
\hline 79 & \multicolumn{2}{|c|}{$\begin{array}{l}\text { Grow PECVD } \mathrm{SiO}_{2} \text { on dummy wafer } \\
\text { (Use parameters from step } 78 \text { ) }\end{array}$} & $\begin{array}{l}\text { Grow } 10 \text { mins then measure the thickness to find } \\
\text { out the grow speed. (It is about } 41.5 \mathrm{~nm} / \mathrm{min} \text { ) }\end{array}$ \\
\hline 80 & \multicolumn{2}{|c|}{$\begin{array}{l}\text { Grow PECVD } \mathrm{SiO}_{2} \text { on device wafers } \\
\text { Grow time: } 75 \mathrm{~min}(3 \mu \mathrm{m} \text { thick layer })\end{array}$} & $\begin{array}{l}\text { PECVD top cladding: } 3 \mu \mathrm{m} \text { (anneal at } 1100{ }^{\circ} \mathrm{C} \text { for } \\
3 \mathrm{~h} \text { has a negligible change to the thickness), grow } \\
75 \mathrm{mins} \text {, about } 40 \mathrm{~nm} / \mathrm{min} \text { in the center (seems } \\
\text { slower than grow a thin layer). } 4.3 \mathrm{~cm} \text { from the } \\
\text { center has thickness up to } 3.4 \mu \mathrm{m} .\end{array}$ \\
\hline $81-87$ & \multicolumn{2}{|c|}{ Standard wafer cleaning* } & \\
\hline 88 & \multicolumn{2}{|c|}{$\begin{array}{l}\text { Annealing of the PECVD } \mathrm{SiO}_{2} \text { : } \\
\text { T: } 1100{ }^{\circ} \mathrm{C}, \mathrm{N}_{2} \text { flow, } 3 \text { hours }\end{array}$} & Use $\mathrm{H} 1$ furnace. \\
\hline
\end{tabular}




\section{Etch micro-ball lens platform}

\begin{tabular}{|c|c|c|c|}
\hline Step & \multicolumn{2}{|c|}{ Process } & Comments \\
\hline 89 & \multicolumn{2}{|c|}{ Measure wafer weight before grow poly-silicon } & \\
\hline $90-96$ & \multicolumn{2}{|c|}{ Standard wafer cleaning* } & $\begin{array}{l}\text { This is only needed if the waiting/loading of } \\
\text { the wafers takes longer than } 4 \text { hours }\end{array}$ \\
\hline \multirow[t]{4}{*}{97} & \multicolumn{2}{|c|}{ LPCVD Poly Silicon layer grow: } & \multirow{4}{*}{$\begin{array}{l}\text { Grow } 1050 \mathrm{~nm} \text { thick layer (needs } 7 \text { hours } \\
\text { including load and unload time) use LPCVD } \\
\text { furnace F2. Deposition rate is } 3.4 \mathrm{~nm} / \mathrm{min} \text {. } \\
\text { Stress: } 30 \mathrm{MPa} \text {. Maximum thickness: } 2.5 \mu \mathrm{m} \text {. }\end{array}$} \\
\hline & Program: senspoly; & $\mathrm{SiH}_{4}$ flow: $50 \mathrm{sccm}$ & \\
\hline & $\mathrm{T}: 590{ }^{\circ} \mathrm{C}$ & Pressure: 250 mTorr; & \\
\hline & Grow time: $6 \mathrm{hrs}$ & & \\
\hline 98 & \multicolumn{2}{|c|}{$\begin{array}{l}\text { Measure wafer weight after grow poly-silicon: } \\
\text { The weight factor of poly-silicon (for } 100 \mathrm{~mm} \text { wafer) } \\
\text { is } 26.5 \mathrm{~nm} / \mathrm{mgr} \text {. }\end{array}$} & $\begin{array}{l}\text { Within one grow, thicknesses are found in } \\
\text { between } 1054 \mathrm{~nm} \text { (close to door) and } 1016 \mathrm{~nm} \\
\text { (close to pump) on different wafer. }\end{array}$ \\
\hline $99-103$ & \multicolumn{2}{|c|}{ Standard wafer cleaning* } & $\begin{array}{l}\text { Step } 1,2,5,6 \text { and } 7 . \text { This is only needed if the } \\
\text { waiting/loading of the wafers takes longer } \\
\text { than } 4 \text { hours. }\end{array}$ \\
\hline $104-105$ & \multicolumn{2}{|c|}{ Apply HMDS adhesion layer** } & \\
\hline 106 & \multicolumn{2}{|c|}{$\begin{array}{l}\text { Coating photoresist Olin Oir 906-17: } \\
\text { Spin program: } 4000(4000 \mathrm{rpm}, 30 \mathrm{sec}) \\
\text { Prebake: hotplate, } \mathrm{T}: 95^{\circ} \mathrm{C} \text {, time } 90 \mathrm{~s}\end{array}$} & Use primus Spinner in wet bench 21 \\
\hline 107 & \multicolumn{2}{|c|}{$\begin{array}{l}\text { Alignment \& exposure Olin 906-17' } \\
\text { Electronic Vision Group EV620 Mask Aligner, } \\
\left.\text { exposure time: } 4 \text { s ( } \mathrm{Hg} \text { lamp: } 12 \mathrm{~mW} / \mathrm{cm}^{2}\right)\end{array}$} & $\begin{array}{l}\text { Vacuum mode, center both stage before } \\
\text { alignment, check pressure should }<-0.8 \text {. After } \\
\text { vacuum contact, Waite } 1 \text { min before expose. }\end{array}$ \\
\hline 108 & \multicolumn{2}{|c|}{$\begin{array}{c}\text { Development Olin Oir resist: } \\
\text { After exposure bake on hotplate } \\
\mathrm{T}: 120^{\circ} \mathrm{C} \text {, time } 60 \mathrm{sec} \\
\text { Development in developer: OPD } 4262 \\
\text { In beaker } 1,30 \mathrm{~s} \text {, then in beaker } 2,15-30 \mathrm{~s}\end{array}$} & Wet bench 21 \\
\hline $109-110$ & \multicolumn{2}{|c|}{ Standard wafer cleaning* } & Step 3 and 6 \\
\hline 111 & \multicolumn{2}{|c|}{$\begin{array}{c}\text { Post bake Olin Oir resist: } \\
\text { On hotplate, T: } 120^{\circ} \mathrm{C} \text {, time: } 10 \mathrm{~min}\end{array}$} & Wet bench 21 \\
\hline 112 & \multicolumn{2}{|c|}{ Inspection by optical microscope } & $\begin{array}{l}\text { A Nikon Microscope which is dedicated for } \\
\text { lithography inspection. }\end{array}$ \\
\hline \multirow[t]{4}{*}{113} & \multicolumn{2}{|c|}{$\begin{array}{c}\text { RIE of polysilicon-590 } \mathbf{~} \text { : } \\
\text { (Use TEtske, clean chamber }+ \text { Styros electrode) }\end{array}$} & \multirow{4}{*}{$\begin{array}{l}\text { Etch time } 50 \mathrm{~s} \text { (for my deep etch mask). } \\
\text { During the etching, after about } 40 \mathrm{~s} \text {, there is } \\
\text { no color change anymore at the bottom of the } \\
\text { etched area. The etch rate is load depend } \\
\text { [Such as, ca. } 360 \mathrm{~nm} / \mathrm{min} \text { for } 95 \% \text { load (4")]. } \\
\text { There are about } 0.7 \mu \mathrm{m} \text { under etch in the } \\
\text { device area. }\end{array}$} \\
\hline & Electrode $\mathrm{T}: 10^{\circ} \mathrm{C}$ & $\mathrm{SF}_{6}$ flow: $30 \mathrm{sccm}$ & \\
\hline & $\mathrm{CHF}_{3}$ flow: $7 \mathrm{sccm}$; & $\mathrm{O}_{2}$ flow: $11 \mathrm{sccm}$ & \\
\hline & Pressure: 100 mTorr; & Power: $60 \mathrm{~W}$ & \\
\hline
\end{tabular}




\begin{tabular}{|c|c|c|c|c|c|}
\hline Step & \multicolumn{4}{|c|}{ Process } & Comments \\
\hline \multirow[t]{6}{*}{114} & \multicolumn{4}{|c|}{$\mathrm{RIE}$ of $\mathrm{SiO}_{2}{ }^{\mathrm{i}}$ : } & \multirow{6}{*}{$\begin{array}{l}\text { Adixen AMS } 100 \mathrm{DE} \text {. } \\
\text { Application: }<5 \mu \mathrm{m} \text { etch depth. } \\
\text { Standard recipe. Etch } 27 \text { mins, for } 13 \mu \mathrm{m} \mathrm{SiO}_{2} \\
=3 \mu \mathrm{m} \text { (up to } 3.4 \mu \mathrm{m} \text { annealed } \mathrm{PECVD} \mathrm{SiO}_{2} \text { ) } \\
\left.+1 \mu \mathrm{m} \text { (annealed TEOS } \mathrm{SiO}_{2}\right)+8 \mu \mathrm{m} \\
\left.\text { (thermal } \mathrm{SiO}_{2}\right)+1 \mu \mathrm{m} \text { (over etch). Clean the } \\
\text { chamber }(30 \text { mins) after every } 20 \text { mins etch. } \\
\text { Etch rate } \mathrm{SiO}_{2}: 0.470-0.530 \mu \mathrm{m} / \mathrm{min} \\
\text { Etch rate } \mathrm{Olin}_{907: \text { ca. } 50 \mathrm{~nm} / \mathrm{min}}\end{array}$} \\
\hline & \multicolumn{2}{|l|}{$\mathrm{C}_{4} \mathrm{~F}_{8}: 20 \mathrm{sccm}$} & \multicolumn{2}{|c|}{ He: 150 sccm; } & \\
\hline & \multicolumn{2}{|l|}{$\mathrm{CH}_{4}: 15 \mathrm{sccm}$} & \multicolumn{2}{|c|}{ ICP: 2800 watt; } & \\
\hline & \multicolumn{2}{|c|}{ He-backside cooling: 10} & \multicolumn{2}{|c|}{ CCP: 350 watt; } & \\
\hline & \multicolumn{2}{|c|}{ Pressure: $8.5 \times 10^{-3} \mathrm{mbar}$} & \multicolumn{2}{|c|}{ Electrode $\mathrm{T}:-10^{\circ} \mathrm{C}$} & \\
\hline & \multicolumn{2}{|c|}{ Substrate height: $120 \mathrm{~mm}$; } & & & \\
\hline 115 & \multicolumn{4}{|c|}{ Find out the etch speed } & $\begin{array}{l}\mathrm{This}_{\mathrm{SiO}_{2} \text { etch faster at the center of the wafer }} \\
\text { (about } 6 \sim 7 \% \text { faster). At } 4 \mathrm{~cm} \text { from the center, } \\
\text { photoresist: } 118 \mathrm{~nm} / \mathrm{min} \text {, Poly-silicon: } \\
38 \sim 39 \mathrm{~nm} / \mathrm{min} \text {, thermal } \mathrm{SiO}_{2}: 480 \mathrm{~nm} / \mathrm{min} \text {, } \\
\text { annealed PECVD } \mathrm{SiO}_{2}: 560 \mathrm{~nm} / \mathrm{min}\end{array}$ \\
\hline \multirow[t]{3}{*}{116} & \multicolumn{4}{|c|}{ Strip off resist in oxygen plasma ${ }^{f}$ : } & \multirow{3}{*}{$\begin{array}{l}\text { Use Tepla300. Barrel Etcher ( } 2.45 \mathrm{GHz} \text { ). } \\
\text { Ultra clean system only (no metals except } \mathrm{Al} \text { ). } \\
\text { This step may not needed, since the resist is } \\
\text { etched away during } \mathrm{SiO}_{2} \text { etch. This step may } \\
\text { grow a few nm thick } \mathrm{SiO}_{2} \text { layer on } \mathrm{Si} \text {. }\end{array}$} \\
\hline & \multicolumn{2}{|c|}{$\mathrm{O}_{2}: 200 \mathrm{sccm}(50 \%)$} & \multicolumn{2}{|c|}{ Power: up to $1000 \mathrm{~W}$; } & \\
\hline & \multicolumn{2}{|l|}{ Pressure: 1 mbar; } & \multicolumn{2}{|c|}{ Time: 30 mins (default) } & \\
\hline $117-121$ & \multicolumn{4}{|c|}{ Standard wafer cleaning* } & Step $1,2,3,5$ and 6 \\
\hline \multirow[t]{15}{*}{122} & \multicolumn{4}{|c|}{ Plasma etching of $\mathrm{Si}(\mathrm{B}-\mathrm{HARS})^{\mathrm{j}}$ : } & \multirow{15}{*}{$\begin{array}{l}\text { Adixen AMS } 100 \mathrm{SE} \text {. } \\
\text { Standard recipe. Etch time } 4 \mathrm{~min} 48 \mathrm{~s} \text {. Etch } \\
19 \mu \mathrm{m} \mathrm{Si} \text {. In case of no poly-silicon on top, } \\
\text { the etch rate of } \mathrm{Si} \text { is about } 3.96 \mu \mathrm{m} / \mathrm{min} \text { for } \\
\text { my deep etch mask. (In case of having } \\
\text { poly-silicon on top, } 100 \% \text { load of a } 100 \mathrm{~mm} \\
\text { diameter wafer, the each rate of } \mathrm{Si} \text { is about } \\
264 \mathrm{~nm} / \mathrm{min} \text { ) }\end{array}$} \\
\hline & Parameters & Etch & & deposition & \\
\hline & Gas & $\mathrm{SF}_{6}$ & & $\mathrm{C}_{4} \mathrm{~F}_{8}$ & \\
\hline & Flow [sscm] & 250 & & 200 & \\
\hline & Time [sec] & 3 & & 1 & \\
\hline & Priority & 2 & & 1 & \\
\hline & $\mathrm{APC}[\%]$ & 100 & & 100 & \\
\hline & CCP LF [watt] & 80 & & 80 & \\
\hline & Pulsed LF ms & $10 / 90$ & & $10 / 90$ & \\
\hline & ICP [watt] & 1500 & & 1500 & \\
\hline & $\mathrm{He}[\mathrm{mbar}]$ & 10 & & 10 & \\
\hline & $\mathrm{SH}[\mathrm{mm}]$ & 200 & & 200 & \\
\hline & Eelctrode $\mathrm{T}^{\circ} \mathrm{C}$ & 10 & & 10 & \\
\hline & Er Oir Olin & $25-50 n$ & $\mathrm{~m} / \mathrm{min}$ & & \\
\hline & Er silicon & $1-5 \mu \mathrm{m}$ & $\min$ & & \\
\hline
\end{tabular}


Fabricate micro-ball lens

\begin{tabular}{|c|c|c|}
\hline Step & Process & Comments \\
\hline $123-127$ & Standard wafer cleaning* & Step $1,2,5,6$ and 7 \\
\hline $128-129$ & Apply HMDS adhesion layer** & Use vapor prime HMDS oven \\
\hline 130 & $\begin{array}{l}\text { Spin coat the first layer of AZ9260: } \\
\text { Pour the AZ9260 on wafer and wait } 20 \mathrm{~s} \text { for the } \\
\text { resist cover most wafer area (good for layer } \\
\text { uniformity). } \\
900-1500-900(1.3 \mathrm{~s})\end{array}$ & $\begin{array}{l}900-1500-900(1.3 \mathrm{~s}) \text { means, the spin is } \\
\text { accelerated with } 900 \mathrm{rpm} / \mathrm{s} \text { to } 1500 \mathrm{rpm}(1.7 \mathrm{~s} \\
\text { acceleration time }) \text { and keep at } 1500 \mathrm{rpm} \text { for } \\
1.3 \text { second and then decelerate with } 900 \mathrm{rpm} / \mathrm{s} \\
\text { to full stop. }\end{array}$ \\
\hline 131 & $\begin{array}{l}\text { Dry the first AZ9260 layer: } \\
\text { Keep wafer at room temperature and on a horizontal } \\
\text { surface for } 20 \mathrm{~min} \text {; } \\
\text { On hot plate: } 60{ }^{\circ} \mathrm{C} 5 \mathrm{~min} ; 100{ }^{\circ} \mathrm{C} 5 \mathrm{~min} ; 60{ }^{\circ} \mathrm{C} \\
1 \mathrm{~min} \text {; } \\
\text { Cool to room temperature (at least } 5 \text { mins). }\end{array}$ & $\begin{array}{l}\text { Pre-heating and pre-cooling is needed before } \\
\text { and after in contact with each hot plat to } \\
\text { prevent cracks in the resist film due to sudden } \\
\text { temperature change. In practice, the } \\
\text { pre-heating and pre-cooling is done by } \\
\text { keeping the wafer approximate } 1 \mathrm{~mm} \text { above } \\
\text { the hot plat with a tweezer for } 20 \mathrm{~s} \text {. The } \\
\text { AZ9260 layer thickness is about } 38 \mu \mathrm{m} \text { on a } \\
\text { flat surface. }\end{array}$ \\
\hline 132 & $\begin{array}{l}\text { Spin coat the second layer of AZ9260: } \\
\text { Quickly pour the AZ9260 on wafer, don't wait. } \\
1000-3000-1000(1 \mathrm{~s})\end{array}$ & $\begin{array}{l}\text { Try to cover the center area (diameter of } 3 \mathrm{~cm} \\
\text { or larger) during pouring. }\end{array}$ \\
\hline 133 & $\begin{array}{l}\text { Dry the second AZ9260 layer and edge bead } \\
\text { removal: } \\
\text { Keep wafer at room temperature and on a horizontal } \\
\text { surface for } 10 \mathrm{~min} \text {; } \\
\text { On hot plate: } 60^{\circ} \mathrm{C} 3 \mathrm{~min} \text {; } \\
\text { Edge bead removal: } 1500 \mathrm{rpm} 30 \text { s with RER600 or } \\
\text { Acetone; } \\
\text { On hot plate: } 100^{\circ} \mathrm{C} 50 \mathrm{~min} \text {; } 60^{\circ} \mathrm{C} 1 \mathrm{~min} \text {; } \\
\text { Cool to room temperature (at least } 5 \mathrm{mins} \text { ). }\end{array}$ & $\begin{array}{l}\text { Pre-heating and pre-cooling is needed before } \\
\text { and after in contact with each hot plat to } \\
\text { prevent cracks in the resist film due to sudden } \\
\text { temperature change. The total AZ9260 layer } \\
\text { thickness is about } 55 \mu \mathrm{m} \text { on a flat surface. }\end{array}$ \\
\hline 134 & $\begin{array}{l}\text { AZ9260 layer rehydration: } \\
\text { Immerse in } 30^{\circ} \mathrm{C} \text { deionized (DI) water for } 15 \mathrm{~min} \text {; } \\
\text { Spin dry the wafer with } 2000 \mathrm{rpm}, 30 \mathrm{~s} \text {, without } \mathrm{N}_{2} \\
\text { flow; Keep the wafer in contact with the cleanroom } \\
\text { air }(\sim 48 \% \text { Hum.) for } 3.5 \text { hours before exposure. }\end{array}$ & \\
\hline 135 & $\begin{array}{l}\text { Alignment \& Exposure of AZ9260': } \\
\text { Use vacuum or hard contact mode. } \\
\text { Dose: } 3 \mathrm{~J} / \mathrm{cm}^{2} \text { (Exposure Time: } 250 \mathrm{~s} \text { ) }\end{array}$ & $\begin{array}{l}\text { Electronic Vision Group EV620 Mask Aligner } \\
\left.\text { (Hg-lamp: } 12 \mathrm{~mW} / \mathrm{cm}^{2}\right) \text {. }\end{array}$ \\
\hline 136 & $\begin{array}{c}\text { Development of } \mathbf{A Z 9 2 6 0 :} \\
\text { Developer: OPD4262, } 27^{\circ} \mathrm{C}, \sim 48 \mathrm{~min}\end{array}$ & $\begin{array}{l}\text { The developer is stirred continually with a } \\
\text { stirring rod in order to have a uniform } \\
\text { development speed over the wafer. }\end{array}$ \\
\hline $137-138$ & Standard wafer cleaning* & Step 3 and 6 \\
\hline
\end{tabular}




\begin{tabular}{|c|c|l|}
\hline Step & Process & Comments \\
\hline 139 & $\begin{array}{c}\text { Waiting time before reflow: } \\
\text { At least } 30 \text { mins }\end{array}$ & To prevent bubble forming during reflow. \\
\hline 140 & $\begin{array}{c}\text { Reflow of } \mathbf{A Z 9 2 6 0 :} \\
\text { On hot plate: } 120^{\circ} \mathrm{C}, 2 \mathrm{~min}\end{array}$ & \\
\hline
\end{tabular}

\section{Dicing}

\begin{tabular}{|c|c|l|}
\hline Step & Process & \multicolumn{1}{c|}{ Comments } \\
\hline 141 & Dicing of a Silicon wafer & Dicing Saw Loadpoint Micro Ace 3 \\
\hline 142 & Clean the chip & Wash with $\mathrm{H}_{2} \mathrm{O}$, and dry with air flow \\
\hline
\end{tabular}

\section{*standard wafer cleaning}

\begin{tabular}{|c|c|c|}
\hline Step & Process & Comment \\
\hline 1 & $\begin{array}{l}\text { Clean in HNO3-1: } \\
\text { beaker 1: } \mathrm{HNO}_{3}(99 \%) 5 \mathrm{~min}\end{array}$ & Wet bench 14 \\
\hline 2 & $\begin{array}{l}\text { Clean in HN03-2: } \\
\text { beaker } 2: \mathrm{HNO}_{3}(99 \%) 5 \mathrm{~min}\end{array}$ & Wet bench 14 \\
\hline 3 & $\begin{array}{l}\qquad \text { Quick Dump Rinse (QDR): } \\
\text { Recipe 1 QDR: } 2 \text { cycles of steps I till III } \\
\text { I: fill bath } 15 \mathrm{sec} \text {; } \\
\text { II: spray dump } 15 \mathrm{sec} \text {; } \\
\text { III: spray-fill } 40 \mathrm{sec} \text {; } \\
\text { IV: end fill } 500 \mathrm{sec} \\
\text { Rinse till the deionized (DI) water resistivity is }>10 \Omega \mathrm{M}\end{array}$ & Wet bench \\
\hline 4 & $\begin{array}{c}\text { Clean in } \mathbf{H N O 3 - 3 a / b : ~} \\
\text { beaker } 3 \mathrm{a} / \mathrm{b}: \mathrm{HNO}_{3}(69 \%) \text {, T: } 95^{\circ} \mathrm{C} \text {, time }>10 \mathrm{~min}\end{array}$ & Wet bench 14 \\
\hline 5 & $\begin{array}{l}\qquad \text { Quick Dump Rinse (QDR): } \\
\text { Recipe 1 QDR: } 2 \text { cycles of steps I till III } \\
\text { I: fill bath } 15 \mathrm{sec} \text {; } \\
\text { II: spray dump } 15 \mathrm{sec} \text {; } \\
\text { III: spray-fill } 40 \mathrm{sec} \text {; } \\
\text { IV: end fill } 500 \mathrm{sec} \\
\text { Rinse till the DI water resistivity is }>10 \Omega \mathrm{M}\end{array}$ & $\begin{array}{l}\text { Wet bench } \\
\text { In case of using the Semitool for } \\
\text { rinsing/drying a single rinsing step } \\
\text { (QDR) is required. }\end{array}$ \\
\hline 6 & $\begin{array}{l}\text { Substrate drying: } \\
\text { Single wafer dryer. Speed: } 2500 \mathrm{rpm}, 60 \mathrm{sec} \text { with } 30 \mathrm{sec} \mathrm{N}_{2} \text { flow }\end{array}$ & Wet bench \\
\hline
\end{tabular}




\begin{tabular}{|c|c|c|}
\hline Step & Process & Comment \\
\hline 7 & $\begin{array}{l}\text { Substrate rinsing/drying Semitool: } \\
\text { - rinse in DI: } 30 \mathrm{sec}: 600 \mathrm{rpm} \\
\text { - Qrinse in DI: } 10.0 \mathrm{M} \Omega ; 600 \mathrm{rpm} \\
\text { - } \mathrm{N}_{2} \text { purge: } 10 \mathrm{sec} ; 600 \mathrm{rpm} \\
\text { - drying 1: } 280 \mathrm{sec} ; 1600 \mathrm{rpm} \\
\text { - drying 2: } 0000-0000\end{array}$ & $\begin{array}{l}\text { This drying step can be used to replace } \\
\text { step } 6 . \\
\text { Wet bench } \\
\text { Apply always a single rinsing step } \\
\text { (QDR) before using the Semitool. Use } \\
\text { dedicated wafer carrier of rinser dryer. }\end{array}$ \\
\hline
\end{tabular}

**Apply HMDS adhesion layer

\begin{tabular}{|c|c|c|}
\hline Step & Process & \multicolumn{1}{c|}{ Comment } \\
\hline 1 & $\begin{array}{c}\text { Dehydration bake: } \\
\text { Dehydration bake at hotplate, T: } 120^{\circ} \mathrm{C}, \text { time: } 5 \mathrm{~min}\end{array}$ & $\begin{array}{l}\text { Wet bench } 21 / 22 \\
\text { Continue immediacy } \\
\text { the priming step! }\end{array}$ \\
\hline 2 & $\begin{array}{c}\text { Priming (liquid): } \\
\text { Primer: HexaMethylDiSilazane (HMDS) } \\
\text { Spin coater program: } 4000(4000 \mathrm{rpm}, 30 \mathrm{sec})\end{array}$ & Wet bench $21 / 22$ \\
\hline
\end{tabular}

This two steps can be replaced by using a vapor prime HMDS oven. T: $150{ }^{\circ} \mathrm{C}$, purge 3 times with alternating $\mathrm{N}_{2}$ and vacuum, then apply HMDS vapor.

\section{Main machines}

a. M-2000 ellipsometer, J.A. Woollam Co.. Spectral range: 245 - $1690 \mathrm{~nm}$.

b. Plasma enhanced chemical vapor deposition (PECVD) machine, PlasmaPro 80 PECVD, Oxford Instruments.

c. Mask Aligner, EVG620, EV Group.

d. Reactive ion etch (RIE) machine, PlasmaTherm 790 series, Plasma-Therm.

e. Step height measurement tool, Dektak 8, Bruker Corporation.

f. A tool for stripping photoresist coatings from the substrate by $\mathrm{O}_{2}$ plasma. TePla 300, PVA Metrology \& Plasma Solutions GmbH.

g. PECVD machine, PlasmaPro 133 PECVD, Oxford Instruments.

h. RIE machine. TEtske, MESA+.

i. RIE machine. AMS $100 \mathrm{DE}$, Adixen.

j. RIE machine. AMS $100 \mathrm{SE}$, Adixen.

k. Dicing Saw Micro Ace 3, Loadpoint Ltd. 


\section{List of Abbreviations}

$\begin{array}{ll}\text { B-HARS } & \text { Bosch high aspect ratio silicon } \\ \text { BPM } & \text { Beam propagation method } \\ \text { CCD } & \text { Charge-coupled device } \\ \text { CMOS } & \text { Complementary metal-oxide-semiconductor } \\ \text { CT } & \text { Computed tomography } \\ \text { DC } & \text { Direct current } \\ \text { DI } & \text { Deionized } \\ \text { FAU } & \text { Fiber array unit } \\ \text { FD-OCT } & \text { Fourier-domain OCT } \\ \text { FDTD } & \text { Finite-difference time-domain } \\ \text { FF-OCT } & \text { Full-field OCT } \\ \text { FWHM } & \text { Full-width-at-half-maximum } \\ \text { GRIN } & \text { Gradient-index } \\ \text { HMDS } & \text { Hexamethyldisilazane } \\ \text { LPCVD } & \text { Low-pressure chemical vapor deposition } \\ \text { MA } & \text { Moving average } \\ \text { MMI } & \text { Multimode interference } \\ \text { MO } & \text { Microscope objectives } \\ \text { MRI } & \text { Magnetic resonance imaging } \\ \text { OCT } & \text { Optical coherence tomography } \\ \text { OI } & \text { Optical isolator } \\ \text { PECVD } & \text { Plasma-enhanced chemical vapor deposition } \\ \text { P-OCT } & \text { Parallel OCT } \\ \text { PSF } & \text { Point spread function } \\ \text { PSS-OCT } & \text { Parallel swept-source OCT } \\ \text { RIE } & \text { Reactive ion etching } \\ \text { RIN } & \text { Relative intensity noise } \\ \text { SD-OCT } & \text { Spectral-domain OCT } \\ \text { SEM } & \text { Scanning electron microscope } \\ \text { SiON } & \text { Silicon oxynitride } \\ \text { SNR } & \text { Signal-to-noise ratio } \\ \text { SOI } & \text { Silicon-on-insulator } \\ \text { SS-OCT } & \text { Swept-source OCT } \\ \text { TD-OCT } & \text { Time-domain OCT } \\ & \\ \text { FI } & \\ \text { FI } & \end{array}$


TE

TEOS

TM

UV

WGM
Transverse electric

Tetraethyl orthosilicate

Transverse magnetic

Ultraviolet

Whispering gallery mode 


\section{Summary}

In the research presented in this thesis, we have focused on modeling, design, fabrication and characterization of a chip-based common-path swept-source optical coherence tomography (SS-OCT) system. Here we summarize the main findings of this work.

The goal of this thesis is to develop an OCT chip with external light source and detector. In chapter 1, a literature survey summarized the achievements and limitations of current chip-based OCT systems. We proposed our chip-based system design, which overcomes some of the limitations. The design we proposed is a common-path OCT system with an on-chip microlens for chip-sample coupling. This common-path design uses the end facet of the waveguide as the reference plane, thus eliminating the need for a space-consuming and dispersive on-chip loop reference arm, thereby reducing the chip size and obviating the need for dispersion compensation. The on-chip micro-ball lens eliminates the need of external optical elements for coupling the light between the chip and the sample, thereby reducing the size and the complexity of the system.

In chapter 2, we presented a mathematical model of the Fourier-domain OCT (FD-OCT) and extended the model to a system with multiple reference reflectors. This FD-OCT model is applicable in both spectral-domain OCT (SD-OCT) and SS-OCT. This model indicates that a system with multiple references results in multiple (possibly overlapping) repeating images. The original sample image can be recovered (from the possibly overlapped images) by deconvolution with the point spread function in the axial direction. We then presented a discussion of the different kinds of noise in an OCT system. The signal to noise ratio (SNR) of a shot noise limited system has been discussed in detail. Finally, an overview of the influences on the SNR by optical losses was given (see Table.2.6.1). This knowledge is particularly useful during the system design and OCT data analysis.

Chapter 3 discussed the design and the characterization of the different optical waveguide elements that are required to realize the proposed OCT chip layout. We described the fabrication process of $\mathrm{SiON}$ waveguide structures. We discussed the losses in a bend structure and presented an adiabatic bend design to minimize the bending loss. The loss of the adiabatic bend is so small that it can be considered to be equal to that of a straight waveguide with the same length. We presented a modified Y junction, which has 
enough tolerance to the fabrication error to be used reliably in our OCT system. The Y junction power ratio has been measured to be $0.506 \pm 0.011$ in the wavelength range of $1170 \mathrm{~nm}$ to $1650 \mathrm{~nm}$. The long wavelength end is limited by the detector (InGaAs) but not by the Y junction. The expected working range of this $\mathrm{Y}$ junction is limited by the material transparency at the long wavelength end, which is about $2.5 \mu \mathrm{m}$.

In chapter 4, we demonstrated the design, fabrication and characterization of an integrated micro-ball lens system that allows for a significant reduction of the divergence angle, in both horizontal and vertical direction, of a light beam radiated from the end facet of an optical chip. The beam half divergence angle of $1544 \mathrm{~nm}$ light was reduced from $12.4^{\circ}$ to $1.85^{\circ}$. The fabrication procedure allows careful tuning of the physical dimensions of the lens. The lens is made of photoresist. The designed fabrication procedure is suitable for mass production. Furthermore, the fabrication strategy is not limited to the SiON waveguide platform as used in this thesis. It can be anticipated that, by carefully choosing suitable etching recipes for the fabrication of the platform and the right adhesion material in between the photoresist and the substrate, the strategy described here can be applied to waveguide technologies that are based on other materials.

In chapter 5, we demonstrated a single channel chip-based common-path SS-OCT system with an integrated micro-ball lens. The common-path design eliminates the space-consuming and dispersive on-chip loop reference arm. Therefore, no dispersion compensation is needed to achieve the light-source-limited axial resolution. The three-port configuration (light source, common-path arm, and detector) enables the use of a wavelength-independent $50 / 50 \mathrm{Y}$ junction which is much less sensitive to fabrication errors compared to a directional coupler. The use of an on-chip micro-ball lens eliminates the need for external optical elements for coupling the light between the chip and the sample. Such a micro-ball lens enables a very short distance of a few hundred $\mu \mathrm{m}$ between the sample and the reference plane, which is an important requirement for common-path OCT. The use of this micro-ball lens leads to a signal enhancement up to $37 \mathrm{~dB}$ for a mirror sample compared to the chip without a lens. The SNR of the system was measured to be $71 \mathrm{~dB}$ with $2.6 \mathrm{~mW}$ of power on a mirror sample at a distance of $0.3 \mathrm{~mm}$ from the waveguide end facet $(50 \mathrm{kHz}$ A-scan rate, averaged over 30 scans). Multiple ghost images caused by additional reference planes (originating from the lens surface) could be largely suppressed using a deconvolution scheme. Finally, cross-sectional imaging of a layered optical phantom, with suppressed ghost image, is demonstrated.

In chapter 6, we discussed how the SS-OCT chip design can be modified to enable multichannel OCT measurements. We presented the design and fabrication results of a common-path based parallel SS-OCT (PSS-OCT) chip followed by suggestions for characterization measurements and for possible improvements in the chip design. Before presenting our PSS-OCT design, we briefly introduced the existing bulky parallel OCT 
(P-OCT) systems and their advantages compared with the single beam scanning system. Several characterization experiments were suggested to investigate the crosstalk between the channels and the effects due to waveguide crossings. After that, we presented a discussion on possible future improvements of the chip design on the following aspects: reducing sampling channel spacing, avoiding waveguide crossings and reducing motion blur.

In conclusion, we believe that by integrating a micro-ball lens onto the chip and using a common-path configuration we have moved a significant step forward in the development of on-chip SS-OCT systems. 


\section{Samenvatting}

Bij het in dit proefschrift beschreven onderzoek hebben we ons gericht op het modelleren, ontwerpen, fabriceren en karakteriseren van een op een chip gebaseerde "common-path" "swept-source" optische coherentietomograaf (SS-OCT). Dit hoofdstuk geeft een samenvatting van de belangrijkste onderzoeksresultaten.

Het onderzoeksdoel van dit proefschrift is de ontwikkeling van een OCT chip met een externe lichtbron en detector. Hoofdstuk 1 geeft een literatuuronderzoek naar de huidig mogelijkheden en beperkingen van OCT systemen. We introduceren het ontwerp van ons op een chip gebaseerd OCT systeem die een aantal van de beperkingen van huidige OCT systemen opheffen Het door ons voorgestelde OCT systeem is een "common-path" OCT systeem waarbij een in de chip geïntegreerde microlens wordt gebruikt voor de koppeling van licht in de chip van en naar het preparaat. In het ontwerp wordt het eind-facet van de golfgeleider gebruikt als referentievlak waardoor het gebruik van een extra golfgeleider lus (die veel ruimte in beslag neemt en bovendien dispersie introduceert) overbodig wordt. Hierdoor wordt de chip kleiner en is er geen dispersie compensatie nodig. Door het gebruik van een geïntegreerde microlens hoeven geen externe lenzen gebruikt te worden om de koppeling van het licht tussen chip en preparaat te realiseren waardoor het OCT systeem compacter en minder complex wordt.

In hoofdstuk 2 presenteren we een wiskundig model dat Fourier-domein OCT (FD-OCT) beschrijft. Het model is uitgebreid voor het beschrijven van OCT systemen waarbij er meerdere referentievlakken zijn en is toepasbaar voor zowel "spectral-domain" en "swept-source" OCT. Uit dit model komt naar voren dat een OCT systeem met meerdere referentievlakken resulteert in een signaal dat bestaat uit meerdere (mogelijk overlappende) herhalende afbeeldingen. Doormiddel van de-convolutie van het signaal met de "point spread function" in axiale richting is het vervolgens mogelijk de oorspronkelijke afbeelding van het preparaat te reconstrueren. Ook wordt in dit hoofdstuk een discussie gevoerd over de verschillende soorten ruis die een rol spelen in OCT. De signaal ruis verhouding in een "shot-noise" gelimiteerd OCT systeem wordt uitvoerig besproken. Tenslotte wordt een overzicht gegeven van de invloed van optische verliezen in de chip op de signaal ruis verhouding (zie Tabel 2.6.1). Deze kennis is met name van belang voor het ontwerp van het OCT systeem en voor de OCT data analyse. 
Hoofdstuk 3 bespreekt het ontwerp en de karakterisatie van de verschillende optische golfgeleider elementen die noodzakelijk zijn om de voorgestelde OCT chip te realiseren. We beschrijven het fabricage proces van SiON golfgeleiders. We bespreken de verliezen die optreden in een gebogen golfgeleider en presenteren een adiabatisch gebogen golfgeleider structuur om deze verliezen te minimaliseren. De daarbij te verwachten verliezen zijn dermate gering dat deze te vergelijken zijn met die van een rechte golfgeleider van dezelfde lengte. Een gemodificeerde Y-splitter wordt geïntroduceerd waarbij de toegestane fabricage toleranties voldoende groot zijn zodat een Y-splitter gebruikt kan worden voor het OCT systeem. De power ratio van de Y-splitter is experimenteel bepaald en bedraagt $0.506 \pm 0.011$ voor golflengten tussen $1170 \mathrm{~nm}$ en $1650 \mathrm{~nm}$. De langste golflengte wordt hierbij beperkt door de gebruikte InGaAs detector en niet door de Y-splitter. De te verwachten langste te gebruiken golflengte is ongeveer $2.5 \mu \mathrm{m}$ en wordt beperkt door absorptie in het materiaal voor langere golflengten.

In hoofdstuk 4 beschrijven we het ontwerp, de fabricage en de karakterisatie van een geïntegreerde microlens die het mogelijk maakt om de divergentiehoek van het licht dat uittreedt uit de optische chip in de horizontale en de verticale richting significant te verkleinen. Voor licht met een golflengte van $1544 \mathrm{~nm}$ wordt de halve divergentie hoek van het uittredende licht verkleind van $12.4^{\circ}$ tot $1.85^{\circ}$. De gebruikte fabricage methode, waarbij de lens gemaakt wordt van fotoresist, maakt het mogelijk de fysische dimensies van de lens zorgvuldig te kiezen. De methode is bovendien geschikt voor massa productie. Ook wordt de fabricage methode niet beperkt tot het gebuik in combinatie met de hier gebruikte SiON golfgeleiders. Het is te verwachten dat, door een zorgvuldige keuze van het ets-proces voor het maken van het platform waarop de lens wordt aangebracht en de juiste keuze van een hechtlaag tussen substraat en fotoresist, de beschreven methode ook voor golfgeleiders van andere materialen kan worden gebruikt.

Hoofdstuk 5 beschrijft een enkel-kanaals SS-OCT chip systeem met een geïntegreerde microlens. Door het gebruik van een gemeenschappelijk referentie en sample arm ("common-path") is een extra golfgeleider lus (die veel ruimte in beslag neemt en bovendien dispersie introduceert) overbodig. Hierdoor is dispersie compensatie niet noodzakelijk en wordt de axiale resolutie slechts beperkt door de gebruikte lichtbron. Het OCT ontwerp, bestaande uit drie poorten (lichtbron, "common-path", en detector), maakt het mogelijk om gebruik te maken van een golflengte onafhankelijke 50/50 Y-splitter. Een dergelijke Y-splitter heeft veel betere fabricage toleranties vergeleken met een "directional coupler". Door het gebruik van een geïntegreerde microlens hoeven geen externe lenzen gebruikt te worden om de koppeling van het licht tussen chip en preparaat te realiseren hetgeen het OCT systeem compacter en minder complex maakt. De microlens makt het bovendien mogelijk om zeer kleine afstanden (een paar honderd micrometer) tussen preparaat en referentievlak te realiseren. Dit is een belangrijke voorwaarde voor het gebruik van SS-OCT. De microlens zorgt voor een signaalversterking van $37 \mathrm{~dB}$ (gemeten bij een 
spiegel als preparaat) ten opzichte van hetzelfde OCT systeem zonder microlens. De signaal ruis verhouding van het OCT-systeem is bepaald op $71 \mathrm{~dB}$ (gemeten bij een optisch vermogen van $2.6 \mathrm{~mW}$ op een spiegel als preparaat op een afstand van $0.3 \mathrm{~mm}$ van de chip, $50 \mathrm{kHz}$ A-scan frequentie, gemiddeld over 30 scans). OCT afbeeldingen laten naast de hoofdafbeelding meerder overlappende afbeeldingen zien die worden veroorzaakt door extra referentievlakken (reflecties aan de microlens). Door toepassing van de ontwikkelde de-convolutie methode was het mogelijk deze extra overlappende afbeeldingen sterk te reduceren. Tenslotte wordt in dit hoofdstuk het OCT-systeem gebruikt om afbeeldingen te maken van een fantoom bestaande uit een aantal optisch verschillende lagen.

In hoofdstuk 6 bespreken we de mogelijkheid om met een aangepaste lay-out van de SS-OCT chip multi-kanaals metingen te realiseren. We bespreken zowel het aangepaste ontwerp als ook de resultaten van de fabricage van de multi-kanaals SS-OCT chip (PSS-OCT). Voordat het ontwerp wordt besproken geven we kort een overzicht van bestaande bulk multi-kanaals OCT systemen en bespreken de voordelen ten opzichte van een scannend enkel-kanaals systeem. Tevens doen we voorstellen voor experimenten om de chip te karakteriseren waar we met name aandacht besteden aan de cross-talk tussen kanalen en de invloed van de in het ontwerp opgenomen kruisende kanaalgolfgeleiders. Ook worden suggesties gedaan voor verbeteringen van de chip-lay-out waarbij specifiek aandacht wordt besteed aan het reduceren van de afstand tussen de kanalen, het voorkomen van kruisende golfgeleiders op de chip en het minimaliseren van bewegings artefacten.

Concluderend zijn wij er van overtuigd dat de in dit proefschrift geïntroduceerde SS-OCT systeem dat gebaseerd is op een optische chip die bestaat uit een combinatie van een geïntegreerde microlens en een gemeenschappelijk referentie en meet arm een significante stap voorwaarts is in de ontwikkeling van chip gebaseerde OCT systemen. 


\section{List of publications}

- $\quad$ L. Chang, N. Weiss, A.G.J.M. van Leeuwen, M. Pollnau, R. M. de Ridder, K. Wörhoff, V. Subramaniam, and J. S. Kanger, (2016) Chip based common-path optical coherence tomography system with an on-chip microlens and multi-reference suppression algorithm. Optics express, accepted.

- $\quad$ L. Chang, M. Dijkstra, N. Ismail, M. Pollnau, R. M. de Ridder, K. Wörhoff, V. Subramaniam, and J. S. Kanger, (2015) Waveguide-coupled micro-ball lens array suitable for mass fabrication. Optics express, 23 (17). pp. 22414-22423. ISSN 1094-4087.

- $\quad$ L. Chang, M. Dijkstra, R. M. d. Ridder, K. Wörhoff, V. Subramaniam, and J. S. Kanger, (2015) Integrated Polymer Micro-ball Lenses for Two-dimensional Divergence Reduction of Light from Single-mode Optical Waveguides. In: Conference on Lasers and Electro-Optics, CLEO/Europe 2015, 21-25 June 2015, Munich, Germany (pp. CK-14.1).

- B. Imran Akça, B. Povazay, L. Chang, A. Alex, K. Wörhoff, R. M. de Ridder, W. Drexler, and M. Pollnau, (2013) Advanced integrated spectrometer designs for miniaturized optical coherence tomography systems. In: Optical Coherence Tomography and Coherence Techniques VI, 12-16 May 2013, Munich, Germany.

- $\quad$ L. Chang, N. Ismail, R. M. de Ridder, M. Pollnau, and K. Wörhoff, (2013) Integrated polymer microlenses for two-dimensional collimation of light from single-mode optical waveguides. In: Conference on Lasers and Electro-Optics, CLEO/Europe 2013, 12-16 May 2013, Munich, Germany (pp. CK-10.6).

- M. Hammer, H.J.W.M. Hoekstra, R. Stoffer, S.M. García-Blanco, M. Maksimovic, L. Chang, and M.A. Sefünc, (2013) OWTNM 2013 : XXI International Workshop on Optical Wave \& Waveguide Theory and Numerical Modelling; Enschede, The Netherlands, April 19 \& 2-, 2013 ; Proceedings. University of Twente, Enschede, the Netherlands. ISBN 9789036535380

- B. Imran Akça, L. Chang, G. Sengo, K. Wörhoff, M. Pollnau, R. M. de Ridder, V. D. Nguyen, J. Kalkman, and A.G.J.M. van Leeuwen, (2012) Integrated AWG spectrometer for on-chip optical coherence tomography. In: 16th European Conference on Integrated Optics, ECIO 2012, 18-20 April 2012, Sitges-Barcelona, Spain.

- B. Imran Akça, N. Ismail, L. Chang, K. Wörhoff, R. M. de Ridder, and M. 
Pollnau, (2012) Towards Raman spectroscopy on a microchip. In: Scientific Exchange, SCIX 2012, 29 September - 4 October 2012, Kansas City, KS, USA.

- B. Imran Akça, L. Chang, G. Sengo, K. Wörhoff, R. M. de Ridder, and M. Pollnau, (2012) Polarization-independent enhanced-resolution arrayed-waveguide grating used in spectral-domain optical low-coherence reflectometry. IEEE photonics technology letters, 24 (10). pp. 848-850. ISSN 1041-1135.

- L. Chang, B. Imran Akça, G. Sengo, K. Wörhoff, R. M. de Ridder, and M. Pollnau, (2012) Polarization-independent optical low-coherence reflectometry with a non-birefringent arrayed-waveguide grating. In: 16th European Conference on Integrated Optics, ECIO 2012, 18-20 April 2012, Sitges-Barcelona, Spain.

- L. Chang, B. Imran Akça, G. Sengo, K. Wörhoff, R. M. de Ridder, and M. Pollnau, (2012) High-performance spectral-domain optical low-coherence reflectometry with an integrated arrayed-waveguide grating. In: Conference on Lasers and Electro-Optics: Science and Innovations, CLEO: S and I, 6-11 May 2012, San Jose, CA, USA (pp. JTh2A.34).

- $\quad$ L. Chang, N. Ismail, R. M. de Ridder, M. Pollnau, and K. Wörhoff, (2012) On-chip reflowed polymer microlenses for collimating light from single-mode optical waveguides. In: 17th Annual Symposium of the IEEE Photonics Benelux Chapter, 29-30 November 2012, Mons, Belgium (pp. 243-246).

- N. Ismail, L. Chang, G. Sengo, R. M. de Ridder, M. Pollnau, and K. Wörhoff, (2012) Polymer microlenses for collimating light from single-mode silicon oxynitride optical waveguides. In: 16th European Conference on Integrated Optics, ECIO 2012, 18-20 April 2012, Sitges-Barcelona, Spain.

- L. Chang, B. Imran Akça, R. M. de Ridder, K. Wörhoff, and M. Pollnau, (2011) Performance Improvement and Birefringence Investigation of Spectral Domain Optical Coherence Tomography Using a Modified Arrayed Waveguide Grating. In: Annual Symposium of the IEEE Photonics Benelux Chapter 2011, 1-2 December 2011, Ghent, Belgium (pp. 241-244). 


\section{Acknowledgements}

I started my $\mathrm{PhD}$ in the Integrated Optical MicroSystem (IOMS) group in 2011. In 2013 the IOMS group was discontinued due to a reorganization of the University of Twente. Then I joined the Nanobiophysics (NBP) group to continue my PhD research. The switching of the group lead to many unwanted changes in the research environment. However, it also brought many fantastic challenges and opportunities to me. I much enjoyed the years as a $\mathrm{PhD}$ candidate. I would like to express my gratitude to all the people that accompanied me throughout the years of my PhD.

For most I would like to thank my promotor Vinod Subramaniam and assistant promotor Hans Kanger. You have welcomed me to the NBP group when I encountered the discontinuity of the IOMS group which made it possible for me to continue my $\mathrm{PhD}$ research. Vinod, I was always attracted by your kind personality. Furthermore, I always felt greatly supported and clearly orientated after a meeting with you. Thank you so much to support and encourage me through many valleys of my PhD. Hans, I really enjoyed the time working together with you. We had so many interesting and fruitful discussions about all kinds of details in the project. What I have learned from these discussions is not limited to particular knowledge but also to be critical to our own results.

I would also like to acknowledge the rest of my graduation committee members: Prof. Hans Hilgenkamp, Prof. Michel Versluis and Prof. Wiendelt Steenbergen from the University of Twente, Prof. Ton van Leeuwen from the University of Amsterdam, Prof. Peter Bienstman from the Ghent University, Prof. Allard Mosk from the Utrecht University for their valuable input and effort spent on reviewing this thesis. Special thanks to Ton, during all the project meetings you have provided me with many valuable feedback on my work which greatly enriched my knowledge about OCT. You also facilitated our collaboration experiments carried out at the Academic Medical Center (AMC) in Amsterdam of which the results largely contributed to Chapter 5.

I am very thankful to Prof. Mireille Claessens and Prof. Jennifer Herek. Mireille welcomed and supported me in the NBP group. Jennifer kindly offered me the space and necessary support in the Optical Sciences (OS) group to relocate my experimental setup after the discontinuity of the IOMS group.

I wish to thank my former supervisor Prof. Markus Pollnau and former daily supervisor Kerstin Wörhoff. You gave me the opportunity to participate to this wonderful project in the IOMS group. I appreciate a lot your contribution during the first two years of my $\mathrm{PhD}$ as well as after you left the University of Twente. Special thanks to Kerstin for her great support and suggestions about the fabrication technology in the cleanroom. I would also like to thank René M. de Ridder. The discontinuity of the IOMS group and your 
retirement never stopped you from a regular support of my research. Your theoretical knowledge made a strong contribution to the OCT model in Chapter 2.

I thank Imran B Akca and Nur Ismail for your great help during my first year as a $\mathrm{PhD}$ student. Imran, you introduced me to the FD-OCT system and made me to understand the working principle. Nur, you have initiated the microlens idea which is modified and developed to become one of the key elements in this research. I also learned so much knowledge about the integrated optics during the discussions and experiments with both of you.

Thanks to Sergio Vázquez-Córdova, Yean-Sheng Yong, Meindert Dijkstra and Nicolás Weiss. Many times we did experiments together and we always shared experiences with each other. These are really valuable memories to me. Thanks to Yean-Sheng, Amin Abolghassemi Fakhree and Harmen Mulder. I enjoyed the coffee/tea break with you and the sometimes very long and diverse discussions about all kinds of topics. Amin, we shared the office for more than two years. I become so used to you that I felt I missed something when you had a day off especially during our tea break time.

Thanks to Liang Ye, Youwen Fan, Kees van der Werf and Sergio. I enjoyed a lot to discuss with you about detailed techniques for astrophotography. I am glad that you also enjoy astrophotography and that we had a few dark, freezing, but happy and fruitful evenings to discover the wonders of the universe. Special thanks to Sergio that you have organized an OS group activity and invited me to give a presentation about astrophotography. I really enjoyed it to share my hobby with others. Thanks to Robert Molenaar. I learned many photography techniques from you.

I wish to thank all the other colleagues from the NBP group and the former IOMS group since you all helped me and inspired my research and social life: Thanks to Martin Bennink, Kerensa Broersen, Ine Segers-Nolten, Ron Gill, Christian Blum, Sylvia Winters, Saskia Lindhoud, Jord Prangsma, Senthil Thangaraj, Arshdeep Sidhu, Kristian Göeken, Maurice van Dalen, Aditya Iyer, Slav Semerdzhiev, Burcu Celikkol, Himanshu Chaudhary, Christian Raiss, Anja Stefanović, Niels Zijlstra, Peter Bosch, Yvonne Kraan, Kirsten van Leijenhorst-Groener and Irene Konings from the NBP group. Thanks to Hugo Hoekstra, Manfred Hammer, Sonia Garcia Blanco, Mustafa Akin Sefunc, Dimitri Geskus, Saara-Maarit Reijn, Laura Agazzi, Edward Bernhardi, Marko van Dalfsen, Brigit Binkhorst, Fehmi Civitci, So Van Pham, Anton Hollink and Gabriel Sengo from the former IOMS group.

I would like to thank all other friends I have met in Enschede and other cities in the Netherlands. Thanks to Prof. Klaus Boller, Bert Bastiaens, Peter van der Slot, Herman Offerhaus, Pablo Muñoz, Gerwin Steen, Jinfeng Mu (穆进峰), Yin Tao (陶寅), Feng Liu (刘峰), Dan Zhu (朱丹), Fei Liu (刘飞), Yanting Chen (陈燕婷), Junwen Luo (罗珺文), 
Kenan Niu (牛珂楠), Xiaohua Li (李小华), Yingnan Zhao (赵英男), Yin Jia (贾银), Mengdi Yang (杨梦迪), Jie Zhao (赵杰), Yali Zhang (张亚莉), Xingwu Sun (孙醒吾), Xiaokui Zhang (张小奎), Zhonghua Chen (陈中华), Jingwei Zhang (张靖微), Xuelong Chen (陈学龙), Quentin (刘东方), Ding Zheng (郑定), Rong Wang (王荣) and all others. It is hard to name them all, but I keep all the memories. Thanks all for sharing great time together, party, BBQ, badminton, traveling and many more.

Last but not least, I wish to dedicate this thesis to my family: to my parents Yunjin Chang (常允金) and Kerong Yuan (袁克荣) as well as to my parents in law Baishu Du (杜 柏树) and Chunyan Wang (王春艳). Thank you for always supporting me and encouraging me. In particular I dedicate this thesis to my wife Ying Du (杜芗). We met, fell in love and got married during my $\mathrm{PhD}$. You were my precious during the past three and a half years and will always be in the future. Your constant support enabled me to complete my research and develop my hobby of astrophotography.

Thank you All, Lantian Chang 
